\title{
Bioactive compounds with antiglioma activity from marine species
}

\author{
Rodion Khotimchenko ${ }^{1}$, Igor Bryukhovetskiy ${ }^{1}$, Maksim Khotimchenko ${ }^{1}$ and Yuri Khotimchenko ${ }^{1,2, *}$ \\ 1 School of Biomedicine, Far Eastern Federal University, 690090 Vladivostok, Russia \\ 2 Laboratory of Pharmacology, A.V.Zhirmunsky National Center of Marine Biology, Far Eastern Branch, \\ Russian Academy of Sciences, Vladivostok, 690950, Russia \\ * Correspondence: khotimchenko.ys@dvfu.ru
}

\begin{abstract}
The search for new chemical compounds with antitumor pharmacological activity is a necessary process for creating more effective drugs for each specific malignancy type. This review presents the outcomes of screening studies of natural compounds with high anti-glioma activity. Despite significant advances in cancer therapy, there are still some tumors currently considered completely incurable including brain gliomas. This review covers the main problems of the glioma chemotherapy including drug resistance, side effects of common anti-glioma drugs, and genetic diversity of brain tumors. The main emphasis is made on the characterization of natural compounds isolated from marine organisms because taxonomic diversity of organisms in seawaters significantly exceeds that of terrestrial species. Thus, we should expect greater chemical diversity of marine compounds and greater likelihood of finding effective molecules with antiglioma activity. The review covers at least 15 classes of organic compounds with their chemical formulas provided as well as semi-inhibitory concentrations, mechanisms of action, and pharmacokinetic profiles. In conclusion, the analysis of the taxonomic diversity of marine species containing bioactives with antiglioma activity is performed noting cytotoxicity indicators and to the tumor cells in comparison with similar indicators of antitumor agents approved for clinical use as antiglioblastoma chemotherapeutics.
\end{abstract}

Keywords: antitumor activity, natural compounds, glioma multiforme, brain tumors, marine species

\section{Introduction}

\subsection{Brain tumors: main limitations of therapeutics}

Despite the substantial success was achieved in the therapy of malignancies within the recent years, there are few tumors that even now are considered absolutely incurable. Among them, the most malignant ones with the fulminant development belong to a group brain tumors generally termed gliomas that were shown to be barely sensitive to chemotherapy. Gliomas are a group of specific brain tumors originated from the glial (or nonneuronal) cells in the central nervous system (CNS). The term "glioma" covers a large and diverse group of the intrinsic tumors with a common classification based on typical microscopic patterns of the originated putative cells along the glial precursor cell lineages. Basically, gliomas are classified into (i) diffuse tumors with extensive infiltrative growth into the brain surrounding parenchyma, and (ii) gliomas showing a more circumscribed growth development ("non-diffuse gliomas") with the signs of pilocytic astrocytomas and ependymomas [1]. Diffuse gliomas were historically diagnosed as expanded astrocytomas (with glioblastoma as most malignant representative), oligodendrogliomas, and oligoastrocytomas (tumors with a mixed astrocytic and oligodendroglial phenotype). The fourth edition of the CNS Tumor Classification of issued by WHO in 2016 defines a large subset of diffuse gliomas based on presence/absence of isocitrate dehydrogenase (IDH) mutation and $1 \mathrm{p} / 19 \mathrm{q}$ co-deletion due to unbalanced translocation $\mathrm{t}(1 ; 19)(\mathrm{q} 10 ; \mathrm{p} 10)$ with the break point around centromere [2]. In adults the most diffuse and malignant gliomas 
belong to one of three molecular categories: (i) IDH-wildtype, (ii) IDH-mutant but 1p/19qnon-codeleted, or (iii) IDH-mutant and 1p/19q-codeleted. Histologically, the IDHwildtype and the IDH-mutant, 1p/19q-non-codeleted diffuse gliomas generally have an astrocytic phenotype, whereas the IDH-mutant, 1p/19q-codeleted gliomas is of an oligodendroglial phenotype. Based on presence/absence of histological features (marked mitotic activity, necrosis, florid microvascular proliferation), diffuse gliomas are graded as WHO grade II (low grade), WHO grade III (anaplastic) or (in case of 1p/19q-non-codeleted tumors) as WHO grade IV (or glioblastoma, also recognized as glioblastoma multiforme or GBM). The modern classification of gliomas is detailed in the work by P. Wesseling and D. Capper [3].

The standard therapeutic strategy of the GBM treatment includes surgical resection with the following radiotherapy and temozolomide- (TMZ-) based chemotherapy. Based on the report issued by the Central Brain Tumor Registry of the United States (CBTRUS), 1 - and 5-year survival rates were 40.2\% and 5.6\% from 2000 to 2015, respectively [4]. Despite the more findings published by the National Cancer Database (NCDB) showing a significant improvement in the 3-year survival rates for glioblastoma multiforme from 8.0 to $10.5 \%(p<0.01)$ in 2004-2013 [5], an overall life prognosis remains poor, which indicates that contemporary therapeutic approaches for GBM are not effective. Although chemotherapy is considered as essential part of the treatment and prevention of malignancies, just a few drugs have been approved for the treatment of gliomas including TMZ, carmustine, lomustine, and bevacizumab. Thus, there is an obvious unmet medical need requiring discovery of the lead compounds with significant anti-glioma activity.

First line of the anti-GBM chemotherapeutics includes alkylating compounds provoking a great diversity of DNA lesions provided by the reactivity of different nucleophilic atoms on the DNA bases. Several different types of alkylation reactions were described. They are mostly presented with either N-alkylated adducts such as N7-methylguanine (N7-meG), N3-methyladenine (N3-meA) and N3-methylguanine (N3-meG), or O-alkylated adducts including O6-methylguanine (O6-meG) and O4-methylthymine (O4meT).

Nowadays, TMZ is one of the most commonly used and well-studied pharmaceutical agents purposed for the brain tumor treatment. This is a monofunctional SN1 type molecule interacting with both oxygen and nitrogen atoms in nucleic acids. It belongs to the second-generation of imidazotetrazinone prodrugs and is selectively converted into active metabolites inside the tumor cells. TMZ shows almost $100 \%$ bioavailability following oral administration and exceptionally good blood-brain barrier permeability, which is important for the brain tumor therapy. TMZ is rapidly absorbed unchanged and then spontaneously converted into 5-(3-methyltriazen-1-yl)imidazole-4-carboximide (MTIC). Then MTIC is broken down with a release of methyldiazonium cation and 5-aminoimidazole4-carboxamide (AIC). AIC does not exert marked activity and is subjected to excretion via kidneys. Methyldiazonium cations show high reactivity and can form methyl adducts at N7 and N3 guanine positions (N7-meG and N3-meG) and N3 adenine site (N3-meA). In addition, they may methylate $\mathrm{O} 6$ guanine residues (O6-meG) [6]. N-methylation induced damages are fixed by the base excision repair (BER) pathway, which involves $\mathrm{N}$ methylpurine-DNA glycosylase providing removal of the $\mathrm{N}$-methylated bases through hydrolyzing the N-glycosidic bonds. The O6-meG alteration type is used to be repaired by O6-methylguanine-DNA methyltransferase (MGMT). If O6-meG is not repaired, it mispairs with thymine instead of cytosine during DNA replication. The post-replication mismatch repair system (MMR) can recognize the O6-methylguanine: thymine mismatched pairs, which excises it introducing strand breaks and leaving the O6-meG.

The greatest problem of the malignant tumor chemotherapy is the cancer drug resistance. It involves several pathways responsible for development of the tumor cell tolerance to the methylating agents such as increased MGMT expression, impairment of MMR or BER pathways induced by genetic and epigenetic changes, and resistance to apoptosis due to the reduced Bax (pro-apoptotic protein) level and increased Bcl-2 (antiapoptotic protein) content [7]. MGMT expression level in glioma cells was shown to 
reversely correlate with their sensitivity to TMZ whereas MGMT depletion in turn increases cell sensitivity to O6-alkylating agents. In addition, mismatch repair system is found to be involved in the recognition and correction of mispaired bases and insertion/deletion loops generated during DNA synthesis. MMR is activated when MGMT is depleted or suppressed by methylation of the gene promoter resulting in enhanced TMZ cytotoxicity. Normally functioning MMR system recognizes O6-meG-thymine mispairs by the MutS and MutL complexes during DNA synthesis. MSH2, MSH3 and MSH6 genes induce formation of those complexes through identification of the small or large insertiondeletion loops and base-base mismatches. This results in removal of the thymine residues. But due to the mispairing properties of O6-meG, thymine is reinserted during the next round of replication that will be again recognized by the MMR system and more strands breaks are introduced. Accumulation of strand breaks can lead to the cell cycle arrest or apoptosis [8]. It was figured out that MMR-deficient cells are mostly resistant to the O6meG induced DNA impairment because they are incapable of suffering apoptosis in response to such lesions generating genomic instability. Therefore, the MMR expression level defines the cell sensitivity to O6-meG pathway. In other words, tumor cells with the high levels of MMR expression have a higher sensitivity to O6-meG-triggered apoptosis in comparison to the cells expressing lower levels [7]. MMR genes provide resistance of glioma cells to alkylating agents via inactivation of somatic mutations and epigenetic changes and hence simultaneously accelerates mutagenesis in resistant clones. Reduced MSH6 gene expression in glioma cells increases their resistance to the alkylating agents resulting in hypermutation leading to genomic instability. The promoter hypermethylation in MLH1 gene may also be linked to the resistance development to alkylating agents and glioma evolution via the similar mechanisms [6].

BER is the main pathway involved in elimination and/or repair of the oxidized, alkylated, and mismatched bases, apurinic/apyrimidinic (AP) sites, and DNA single strand breaks produced by reactive oxygen species (ROS), ionizing radiation and alkylating agents. Therefore, BER pathway provides repairment of such main lesions as N7-meG and N3-meA induced by chemotherapy with TMZ. Lesion-specific glycosylases, such as MPG, recognize impaired bases and hydrolytically break the $\mathrm{N}$-glycosidic bonds, thus generating an AP sites. Then AP endonuclease (APE-1) cleaves the phosphodiester backbone on the 5' side of the AP site leaving the 3'-OH and 5'-deoxyribose phosphate ends at the DNA strand break. The terminal residue is removed leaving a nucleotide gap. The repair is performed through a short patch BER mechanism that includes replacement of one nucleotide or a long patch BER with a gap filling composed of 2-10 nucleotides [9]. The DNA polymerase $\beta$ in coordination with XRCC1-Ligase III complex complete the repair process. Ineffective functioning of the DNA damage repair mechanisms results in development of the cell cycle arrest usually resulting in cell death. Therefore, DNA damage associated with the complex enzymatic responses may lead to apoptosis, autophagy, necrosis, and other forms of cell death [10].

Apoptosis is a caspase-dependent programmed cell death usually manifested by distinctive morphological and biochemical hallmarks, such as membrane blebbing, cell shrinkage, nuclear condensation and fragmentation, mitochondrial fragmentation, and caspases activation [11]. Apoptosis reaction can be a result of either death receptor-independent (intrinsic or mitochondrial) or dependent (extrinsic) pathways. The mitochondrial pathway is intermediated by the Bcl-2 protein family composed of sets of both antiapoptotic and pro-apoptotic proteins regulating the small molecule pathways including cytochrome $\mathrm{C}$, which trigger caspase cascades through the mitochondrial permeability transition pore. Cells with induced apoptosis reaction contain higher levels proapoptotic proteins such as Bax that damage mitochondria and lead to the release of cytochrome $\mathrm{C}$ to the cytosol with caspase activation and following cell death. The extrinsic pathway initiated by the death receptor activation involves the maturation of caspase-8. Both pathways contribute to a final joint mechanism of the caspase-3 activation [12]. One of the main mechanisms of the cancer drug resistance is an apoptosis evasion that can be realized through the reduced caspase function, impaired death receptor signaling, and induced 
misbalanced between pro-apoptotic and anti-apoptotic proteins, such as Bcl-2 family and p53. Autophagy is a caspase-independent process is manifested with increased autophagic vacuole number in the cytoplasm, which is complemented by the degradation of Golgi apparatus and endoplasmic reticulum. These alterations inevitably result in the nucleus destruction [12]. Autophagy is considered a universal process that can provide both cell death and cell survival effects depending on the cellular content. The cell death mechanism is commonly activated when apoptotic mechanisms are suppressed as it works as a reverse mechanism of the cancer death eradication. In other studies autophagy was described as a pro-survival response that is possibly contributes to the development of the TMZ resistance in glioma cells.

Taking to account the abovementioned mechanisms of the drug cancer resistance development, the following ways to increase efficiency of the anti-glioblastoma chemotherapy may be pointed out including MGMT level modulation and inhibition of the BER pathway via poly(ADP-ribose) (PARP) inhibition, which is known to take part in detecting and signaling the DNA damage generated by methylating agents. Other approaches include blockage of the APE endonuclease activity; modulation of the expression and activity of the regulatory elements responsible for the proper cell functions and apoptosis such as the Bcl-2 protein family, p53 protein, inhibitor of apoptosis proteins or the receptor tyrosine kinases (e.g., EGFR); inhibition of molecular targets related to apoptosis and autophagy resistance pathways, such as the mTOR, PI3K, and Akt.

Another serious problem of the chemotherapy against glioblastomas is linked to the development of severe adverse effects induced by the use alkylating agents. They include hematotoxicity, myelosuppression, hepatotoxicity, cerebral edema, interference in normal blood flow, blood clots, coronary heart disease attacks and peripheral artery disorders, gastrointestinal lesions and bleeding as well as allergic reactions [13]. All these substantial limitations of the up-to-date methods of chemotherapy require development of the novel pharmaceuticals with high efficiency and low toxicity with reduced risk of adverse effects providing favorable forecast of the disease outcome.

Discovery of the new chemical compounds exerting pharmacological effects, in particular, showing anti-glioma effects actually a sequential essential process of construction of the new pharmaceuticals which should be more effective regarding each specific tumor type. Due to the enormous diversity of the tumor genotype an antitumor therapy based on the use of only one drug is less effective for elimination of the tumor cells in comparison with a joint administration of two and more pharmaceuticals. Such combinations are in theory more promising approach for eradication of the high tumor cell number the use of monotherapy [14]. The new approaches discovering combinations of multiple inhibitors have been proposed along with the identification of key driver mutations that are specific to each patient.

\subsection{Molecular targets for the anti-glioma drug discovery}

Malignant brain tumors can be referred to as "primary" or "secondary" GBM depending on if they arise de novo or evolve by progression from a lower-grade glioma. These clinical disease forms bear different molecular markers that may include telomerase reverse transcriptase (TERT) promoter mutation, amplification of the epidermal growth factor receptor (EGFR) gene, phosphatase and tension homolog (PTEN) tumor suppressor gene mutation for primary GBMs. TP53 and IDH1/2 mutations are usually found in secondary GBMs [15]. EGFR amplification in primary GBM is often associated with high expression of EGFRvIII, a ligand-independent constitutively active mutant of EGFR, capable of persistently activating the phosphatidylinositol 3-kinase (PI3K)/v-akt murine thymoma viral oncogene homolog (AKT) signaling pathway that promotes the survival of the glioma cells. The genetic mutations of PTEN down-regulate the AKT signaling pathway. The secondary GBM, that is usually associated with tumor protein p53 (TP53) mutations, consistently exhibits the genetic mutation of isocitrate dehydrogenase 1 (IDH1) down regulating the hypoxia-inducible factor 1-alpha (HIFA). A series of oncogenic pathways in GBM including the p53, the retinoblastoma tumor suppressor (RB), and the receptor 
tyrosine kinases/RAS/phosphatidylinositol 3 kinase (RTK/RAS/PI3K) pathways in most cases are activated [16]. These genetic alterations promote cell proliferation and enhance cell survival capacity, thus making tumor cells to escape from cell-cycle checkpoints, senescence, and apoptosis. Beyond genetic abnormalities, epigenetic alterations such as hypermethylation of the pro-apoptotic genes and tumor suppressor genes, and hypomethylation of genes that are normally silenced such as MMP9 gene (related with invasion) has been described in GBM [17]. The current drugs that specifically target the tyrosine kinase activity of EGFR or selectively inhibit the mammalian target of rapamycin (mTOR), a PI3K/AKT downstream signal transducer showed little efficacy in treatment of primary glioblastoma [18]. Therefore, it may be concluded that the most promising approach for suppression of the glioblastoma development involves concurrent inhibition of multiple signaling pathways affecting molecular targets playing pivotal role in the survival and progression of tumor cells.

Characteristic sign of many human cancer types is disturbed cell cycle regulation affecting various regulatory pathways and intracellular process such as proliferation, differentiation, DNA repair, and apoptosis leading to genetic instability, tumorigenesis, and malignancy. Cell cycle abnormalities are generally caused by hyperactivation of cyclindependent kinases (CDKs) belonging to the serine/threonine-specific protein kinase family that consists of a couple of dozens of members exhibiting conserved catalytic domains and showing similar activation mechanisms through the binding of a regulatory subunit (usually a cyclin) and post-translational modifications. Abnormal regulation of the CDK4and CDK6-cyclin D-INK4-retinoblastoma protein $(\mathrm{Rb})$ signaling pathway is the one among the most common aberrations noted in many human cancers, in particular, in GBMs, which cells shows excessive CDK4/CDK6 activation [19]. Therefore, CDKs are commonly used as targets for antitumor agents as well as other protein kinases involved into cell cycle regulation pathways.

The new findings were published within the recent years indicating the aberrant activation of the specific molecular pathway plays crucial role in progression, recurrence, and invasion of the glioma tumors. A group of molecular signals suggested to be important for glioma cells includes PI3K/AKT, mitogen-activated protein kinase (MAPK), and Wingless (WNT)/b-catenin pathways. PI3K/AKT pathway was shown to be activated in glioma and this activation of such signaling is essential for survival, proliferation, invasion of cells and oncogenesis in glioma cells [20]. Akt is phosphorylated following the PI3K activation. This phosphorylated Akt promotes cell survival and proliferation, as well as other malignant properties through phosphorylating downstream targets including anti-apoptotic protein Bad and transcriptional factors forkhead box protein (FOXO) and NF-kB. Moreover, PI3K/AKT signaling is essential for the glioma tumor growth and it increases glioma cell resistance to apoptosis induced by various stimuli. PTEN deletion (i.e. phosphatase and tensin homology deleted on chromosome 10) or mutation is commonly noted lesion in various cancer types leading to activation of the PI3K/AKT pathway. PI3K/AKT pathway targeting has also been shown to be an effective way providing negative influence on glioma [20]. Therefore, suppressed activation of the PI3K/AKT pathway is thought as one of the mechanisms inhibiting growth of glioma. A protein chaperon Hsp90 providing correct folding, stability, and activation of its client proteins maintains stability and normal functioning of various signal proteins and plays a role in cell proliferation, growth, and survival. One of the serine-threonine kinase is Akt that is also referred to as protein kinase B, is shown to be closely associated with the growth factorinduced signaling pathway. Akt stimulates the growth factors and cytokines and is also recruited from cytosol to plasma membrane and then phosphorylated at two key regulatory sites, Thr308 and Ser473, by 3-phosphoinositide-dependent protein kinase-1 (PDK1). Akt generally binds to the HSP90 protein, which is required for the proper function of Akt as they form a chaperone-substrate protein complex. Reduction of the mutual Hsp90/Akt binding usually results in the Akt inactivation. Inhibition of the Hsp90/AKT complex formation with the following suppression of the и PI3K/AKT signaling pathway can be considered as a promising way for therapeutic inhibition of the glioma growth [21]. 
Checkpoint kinase 1 (Chk1) is another promising target for glioma therapy. Chk1 belongs to a group of Ser/Thr protein kinase involved into the pathways controlling the $\mathrm{G} 2 / \mathrm{M}$ phase of the cell cycle. Increased expression of the Chk1 is typical for major part of the cancer types due to oncogenic activation and constant replicative stress. Inactivation of the Chk 1 is a promising mechanism of the cancer therapy because it contributes to genomic instability, chromosome catastrophe, and cancer cell death. Cdc25C is one of the well-defined target for Chk1 regulation. Chk1 phosphorylates the phosphatase Cdc25C at the Ser216 site promoting Cdc25C binding to the molecular chaperone 14-33 protein. The 14-3-3 protein then sequesters Cdc25C in the cytoplasm and may lead to the G2/M arrest of malignant human glioma cells [22]. Chk1 along with the ataxia-telangiectasia-mutatedand-Rad3-related kinase (ATR) are two key components of the replication stress response. ATR/CHK1 signaling pathway prevents the entry of cells harboring damaged or incompletely replicated DNA into mitosis when the cells are exposed to DNA damaging agents. That is especially typical of cells with a deficiency in the cell-cycle G1 phase checkpoint, which is frequently found in tumors (for example, loss of TP53). ATR/CHK1 also suppresses replication stress reaction induced by oncogene activation to a less toxic level. Consequently, survival of the cancer cells with high levels of oncogene-induced replication stress mostly depends on the ATR or CHK1 functions. These properties of CHK1 and ATR make them promising target structures for discovery of the new anticancer drugs $[23,24]$. Since Chk1 plays a central role in mediating the cellular response to DNA damage, the Chk1 inhibitors should be considered as potential antitumor agents inducing increased DNA damage similarly to the standard cytotoxic or radiation therapy.

Tumor glycolytic enzymes hexokinase 2 (HK2), 6-phosphofructo-2-kinase/fructose2,6-bisphosphatase (PFKFB3), pyruvate kinase M2 (PKM2), and lactate dehydrogenase 5 (LDH5) were also found as promising targets in the discovery of the novel anticancer pharmaceuticals. Increased glycolysis level was confirmed to be necessary for the fast and unlimited cell proliferation in tumors and may serve as marked sign of the glioma metabolism. In addition, glycolytic enzymes such as HK2, PFKFB3, PKM2, and LDH5 demonstrate increased activity I the glioma cells and supposed to be preferentially used by cancer cells [25-28].

\subsection{Marine compounds as a source of the new anti-glioma agents}

One of the sources for the prototypes of the new medicines with antitumor activity is a group of natural products (NPs), in particular, NPs of marine origin. Approximately $80 \%$ of the approved chemotherapeutic drugs, and more than $50 \%$ of all drug used in clinical practice are thought to be based on the bioactive NPs [29]. In the numerous drug discovery studies NPs have been a prime source of pharmacologically active agents purposed for the treatment of many cancer forms offering a promising opportunity for evaluation of new chemical classes of anticancer drugs [30].

Macrotaxonomical diversity of marine species is substantially higher than biodiversity of the terrestrial life forms. Among 35 animal phyla that are taxonomically identified today, 34 are found in the marine environment, and many of them inhabit only aqueous media [31]. During the much longer evolution of the marine species that lasted for hundred million years, the harsh oceanic conditions such as wide temperature range, salinity, great pressure as well as a risk of predator attacks contributed to the production of a high variety of molecules bearing unique properties in terms of diversity and structural and functional features. At present time point many marine species including bacteria, cyanobacteria, fungi, microalgae, seaweeds, marine sponges, soft corals, sea fans, sea hares, nudibranchs, bryozoans, tunicates, sea cucumbers and starfishes have been investigated for the presence of compounds with anti-cancer activities $[32,33]$. Despite the material supply and availability poses a great problem for the drug development based on marine products, which are usually obtained from natural sources in very small amount, modern methods of the total chemical synthesis or semisynthesis provide a reasonable solution. Biotechnological technologies are supposed to me more time and resource consuming can be implemented for such purposed in a form of large-scale fermentation of the producer 
microorganisms or the cultivation of marine invertebrates [34]. Nowadays numerous marine-based drugs initially obtained from NPs including spongian nucleoside cytarabine and spongian macrolide eribulin mesylate, brentuximab vedotin (an antibody-drug conjugate brentuximab with the monomethyl auristatin E (MMAE), which is a synthetic ana$\log$ of dolastatin-10, produced by marine cyanobacteria) and second antibody-drug conjugate, polatuzumab vedotin, which consists of a CD76b-targeting antibody and MMAE, ascidian alkaloid trabectidine (ET-743) and ascidian depsipeptide plitidepsin are in use in the treatment of lymphocytic leukemia, anaplastic large T-cell systemic malignant lymphomas and B-cell lymphomas, Hodgkin's and non-Hodgkin lymphomas, multiple myeloma, metastatic breast cancer, soft tissue sarcoma and ovarian cancer [35]. Determination of the pharmacophore structure providing anticancer activity opens an opportunity to obtain the new modified compounds with higher potency, more selective activity, and less toxicity.

There are compounds with anti-glioma activity belonging to the various classes of chemical substances was discovered. Pharmacological properties of these substances will be discussed in the following chapters on the present review article.

\section{Alkaloids}

Alkaloids are cyclic compounds containing one or more nitrogen atoms in their cycle or side chain that demonstrate weak alkali properties. Pharmacognosy classification designate two classes of these compounds depending on the nitrogen atom location, which may be in the circle or in the side chains. First group referred as heterocyclic alkaloids (or typical alkaloids) structurally have the nitrogen as a part of a cyclic ring system. They are subdivided into several subgroups on base of the ring structure containing nitrogen atom. These circular structures may be presented with such heterocycles as pyridine and piperidine, pyrrol and pyrrolidine, tropane, quinoline, isoquinoline, phenanthrene, indole, purine, imidazole, pyrrolizidine, indolizidine, aporphine, and quinolizidine [36]. Second group generally termed non-heterocyclic alkaloids (or atypical alkaloids) have a nitrogen atom which is not a part of any ring system. Nowadays, steroidal and terpenoid groups are considered as separate classes or along with glycosides. Main natural sources of alkaloids belong to the plant kingdom, and mainly to higher plants. However, within the recent years the number of alkaloid molecules found in the marine sources is gradually growing. Those species include marine bacteria including cyanobacteria (or blue-green algae), actinomycetes, marine-derived fungi, marine algae, bryozoans, starfishes, holothurians, ascidians, and marine sponges [37]. Bioactive alkaloids as well as officinal medicine alkaloids exert various pharmacological effects including antitumor properties [38]. Alkaloids compounds demonstrating antiglioma activity are listed below with anticancer mechanisms given in details.

Imidazolone and indole alkaloids. A new alkaloid named zorrimidazolone (molecular formula $\mathrm{C} 11 \mathrm{H} 13 \mathrm{~N} 3 \mathrm{O} 4$ ) isolated from the Mediterranean stolidobranch ascidian Polyandrocarpa zorritensis was shown to exert cytotoxic effects against $\mathrm{C} 6$ cells resulting in $60 \%$ decreased viability when administered at $250 \mu \mathrm{M}$ for 48 hours under in vitro conditions. IC50 for zorrimidazolone providing inhibiting effects against C6 cells was within the micromolar range (Table 1), which is generally considered as moderate cytotoxic activity [39]. This new metabolite belongs to the 2-aminoimidazolone class of marine metabolites, which have been predominantly isolated from Axinella and Agelas marine sponges and very rarely found in the ascidians. The compounds from this class isolated from ascidians are the $\mathrm{N}, \mathrm{N}$-dimethylaminoimidazolone found in Dendrodoa grossularia [40] and the polyandrocarpamines A and B found in a Fijian Polyandrocarpa sp. [41]. Two monoindole alkaloids, 3-indolylglyoxylic acid and its methyl ester that were also found in Polyandrocarpa zorritensis demonstrated weaker cytotoxic activity toward the C6 cells, which is selective and depending on the concentration. Structurally close compound 4hydroxy-3-methoxyphenylglyoxylic acid methyl ester, which does not belong to the alkaloid class, did not affect C6 cell viability. According to the author conclusion, 
zorrimidazolone may be of interest for the development of potential antiproliferative molecules against gliomas.

Table 1. The structure and inhibitory activity of marine metabolites from ascidian Polyandrocarpa zorritensis against glioma C6 line [39].

\begin{tabular}{|c|c|c|}
\hline Compound & Structure & $\mathrm{IC}_{50}, \mu \mathbf{M}^{*}$ \\
\hline Zorrimidazolone & & $155 \pm 13$ \\
\hline
\end{tabular}

3-indolylglyoxylic acid<smiles>O=C(O)C(=O)c1c[nH]c2ccccc12</smiles>

3-indolylglyoxylic acid methyl ester<smiles>COC(=O)C(=O)c1c[nH]c2ccccc12</smiles>

Methyl

2-(4-hydroxy-3-

methoxyphenyl)-2-oxoacetate<smiles>COC(=O)C(=O)c1ccc(O)c(OC)c1</smiles>

${ }^{*} \mathrm{IC}_{50}$ values are expressed as mean \pm SEM.

Indol alkaloids meridianins isolated from ascidians Aplidium meridianum [42] and variolin B found in Antarctic sponge Kirkpatrickia varialosa [43] became prototypes of the novel synthetic derivatives as well as hybrid molecules meriolins obtained from those compounds. The natural meridianins A, B, C, D, E, F, and G are brominated and/or hydroxylated 3-(2-aminopyrimidine)-indoles differing in the bromine and/or hydroxyl substitution (Figure 1) [44,45]. Meridianins were shown to inhibit various protein kinases, such as CDKs, glycogen synthase kinase-3, cyclic nucleotide-dependent kinases, and casein kinase 1 [46] playing important role in the cancer cell lifecycle. They also prevent cell proliferation and induce apoptosis due to their ability to penetrate cellular membrane and disturb activity of kinases responsible for cell division and death. Meridianin E was 
experimentally proved an effective protein kinase inhibitor with high selectivity regarding CDK1 and CDK5 [46]. One of the meridianin C derivatives substituted at the C-5 position was found to be strong and selective inhibitor of pim kinases (including pim-1, pim2 , and pim-3 overexpressed in various cancer cell types) with IC50 values within a nanomolar concentration range [47].<smiles>[R2]c1c([R])c([R])c2c(-c3ccnc(N)n3)c[nH]c2c1[R]</smiles>

\author{
Meridianin A: R1 =OH, R2 =R3 =R4 $=\mathrm{H}$ \\ Meridianin B: R1 =OH, R2 =R4 =H, R3 =Br \\ Meridianin C: R1 =R3 =R4 =H, R2 =Br \\ Meridianin D: R1 =R2 =R4 $=\mathrm{H}, \mathrm{R} 3=\mathrm{Br}$ \\ Meridianin E: R1 =OH, R2 =R3 =H, R4 =Br \\ Meridianin F: R1 =H, R2 =R3 =Br, R4 =H \\ Meridianin G: R1 =R2 =R3 =R4 =H
}

Figure 1. The chemical structure of meridianins A-G [44].

Variolins (A, B, deoxyvariolin B, and N(3')-methyl tetrahydrovariolin B) are natural marine alkaloids possessing an uncommon pyrido[3',2':4,5]pyrrolo[1,2-c]pyrimidine skeleton (Figure 2) [43]. Variolin B exerted anticancer activity on the P388 murine leukemia cell line with an IC50 value $716 \mathrm{nM}$. Variolin A and N(3')-methyl tetrahydrovariolin B displayed significantly weaker activity against these cancer cells. Variolin B had been shown to induce apoptosis is highly potent cytotoxic activity against various human cancer cell lines including the ones with overexpressed level of cell efflux pump p-glycoprotein (PGP). Variolin B and deoxyvariolin B were experimentally confirmed to exert strong cytotoxic activity against various cancer cell lines with IC50 values varying from 50 to 100 $\mathrm{nM}$. Both compounds were noted to inhibit the histone $\mathrm{H} 1$ phosphorylation mediated by cyclin E-CDK2, cyclin A-CDK2, cyclin B-CDK1, cyclin H-CDK7, and cyclin D-CDK4, with IC50 values in the micromolar range [48].<smiles>[R]c1ccnc2c1c(-c1ccnc(N)n1)c1ccnc(N)n12</smiles>

Variolin $\mathrm{B}: \mathrm{R}=\mathrm{OH}$

Deoxyvariolin B: $\mathrm{R}=\mathrm{H}$

Figure 2. The chemical structure of variolin B and deoxyvariolin B.

A new CDK inhibitory scaffold with antitumor activity has been composed by combining the common features of meridianins and variolins. Thus, the new class of synthetic 7-azaindole-containing analogues have been designed and the term "meriolin" has been 
coined to describe this hybrid structure $[49,50]$. Meriolins exhibit better antiproliferative and proapoptotic properties in cell cultures than their "inspirational parent" molecules. Meriolins was shown to exert significant inhibiting effects against CDKs and possess antiproliferative and pro-apoptotic activity in human cancer cell lines in vitro [50]. In particular, phosphorylation at CDK1-, CDK4-, and CDK9-specific sites has been shown to be counteracted by meriolins in neuroblastoma SH-SY5Y [49].

19 different meriolin structures have been investigated in such human glioma cell lines as anaplastic astrocytoma SW1088 and glioblastoma U87. All mentioned meriolins efficiently decelerated cell growth rate within IC50 range from $1 \mathrm{nM}$ to $1 \mu \mathrm{M}$. Meriolin types 3,5 , and 15 provoked proliferation rate reduction in both glioma cell lines. IC50 for SW1088 cells were 34, 32, and 46 nM, respectively, whereas these values for U87 were 76, 18.4, and $5.1 \mathrm{nM}$, respectively, (Table 2). Meriolin types 5 and 15 showed the strongest antiproliferative activity via induction of the cell cycle block and apoptosis in glioma cells [51].

Table 2. The structure and cytotoxic effects of meriolins against glioma cell lines [51].

\begin{tabular}{lll}
\hline Compound & \multicolumn{2}{c}{ IC50 (nM) } \\
\hline Meriolin 3 Structure & SW888 \\
\hline
\end{tabular}

Meriolin 5<smiles>CCCc1cccnc1-c1c[nH]c2nccnc12</smiles>

In the in vivo experiments meriolin 15 inhibited proliferation of the glioma cells, induced apoptosis and reduced the number of non-differentiated tumor cells in the U87 glioblastoma xenograft model in nude mice. The results suggested that meriolin 15 inhibits DK7/CDK9 consequently decreasing RNA polymerase II and its phosphorylation that results in downregulation of the survival factor Mcl-1, thereby allowing the activation of proapoptotic factors (Noxa, Bim, etc.) [51]. The cytotoxic effect of meriolins have been also investigated in the rat primary proliferating astrocyte and neuron cultures. Meriolin type 5 and 15 demonstrated antiproliferative and pro-death activities. IC50 of the meriolin 15 is $7.8 \mathrm{nM}$ in astrocytes and $4.7 \mathrm{nM}$ in neurons. Therefore, meriolins provide extremely 
potent antiglioma activity and at the same time exert strong toxic effect in healthy astrocytes. This requires further investigations focused on development of other derivative with less toxicity regarding normal healthy cells.

Fascaplysins. Fascaplysin is a red pigment and bis-indole alkaloid (12, 13-Dihydro13-oxopyrido[1,2-a:3,4-b'] diindol-5-ium chloride) was initially isolated from the Fijian sponge Fascapfysinopsis sp. in 1988 and characterized as the novel 12H-pyrido[1,2-a:3,4$\mathrm{b}^{\prime}$ ]diindole ring system (Figure 3), which was considered as a unique among natural products [52]. Later fascaplysin and some related compounds homofascaplysin A and 3-bromohomofascaplysin A were discovered and isolated in ascidian Didemnum sp. [53]. Then synthesis of fascaplysin and its derivatives was elaborated in a relatively short period and this process is still ongoing $[54,55]$.

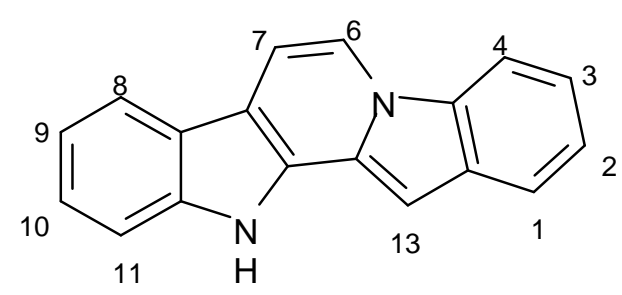

Figure 3. Structure of 12H-pyrido[1,2-a:3,4-b']diindole.

Fascaplysin exerts various biological activities including selective kinase 4 (CDK-4) inhibition, DNA binding, and antiangiogenic effects [56]. Fascaplysin was found to exert cytotoxic effects in panel with at least 36 cancer cell lines with IC50 in a concentration range $0.6-4 \mu \mathrm{M}[54,57-59]$. It was also shown that fascaplysin and its derivatives are quite efficacious in in vivo mouse tumor models against a human colon carcinoma HCT-116, and human non-small-cell lung carcinoma NCI-H460 [54], human malignant melanoma A375 [58], and a murine sarcoma S180 via induction of apoptosis and antiangiogenesis [57].

The molecular mechanism of fascaplysin-induced apoptosis is directly linked to activation of the caspase-3, caspase-8, and caspase-9 pathways, cleavage of Bid, release of cytochrome $\mathrm{C}$ into cytosol and downregulation of the Bcl-2 level in cancer cells. Fascaplysin was also shown to block vascular endothelial growth factor (VEGF), inhibit proliferation, and induce apoptosis of human umbilical vein endothelial cells (HUVECs) [56]. TNF and TNF receptor superfamily in HUVECs and hepatocarcinoma cells BeL-7402 cab be regulated by fascaplysin resulting in the tumor necrosis-related apoptosis-inducing ligand-(TRAIL)-induced apoptosis leading to activation of caspases 3 and 9 and Bid decrease [60]. Fascaplysin was noted to induce high cytotoxicity against small cell lung cancer (SCLC) cells resulting in the cell cycle arrest in G1/0 at lower concentration of active compounds and in S-phase at the higher fascaplysin level. Its high cytotoxic activity against these cancer cells is due to multiple routes of action, affecting topoisomerase I, integrity of DNA and generation of reactive oxygen species (ROS) [57].

Under in vitro conditions the anticancer activity of fascaplysin results in reduced expression of CDK4, cyclin D1 and downregulation of the CDK4-specific Ser795 retinoblastoma protein $(\mathrm{Rb})$ phosphorylation in HeLa cell line. Apoptosis induced the fascaplysin is related to the activation of the effector caspases, migration of cytochrome $\mathrm{C}$ into cytosol, and reduced Bcl-2 expression. Cytotoxic effects of fascaplysin were observed in chemosensitive promyelocytic HL-60 cancer cells as it activates both pro-apoptotic events like PARP-1 cleavage/caspase activation and triggered autophagy as shown by the increased expression of LC3-II, ATG7 and beclin [61]. It should be emphasized that fascaplysin demonstrates significant anticancer activity in non-small cell lung cancer (NSCLC) and small cell lung cancer (SCLC) cell lines that is not depending on the CDK4 pathway thus suggesting the direct effects on the DNA function and transcription of various proteins 
[59]. Another mechanism of antitumor activity was found to be related in increase of the phosphorylation of AKT/PKB and adenosine monophosphate-activated protein kinase (AMPK) that play a key role in anti-apoptotic or pro-survival pathways in cancer [58]. Fascaplysin in addition was shown to abolish phosphorylation of mTOR, 4EBP1, and p70S6K1 thus triggering the cap-dependent translation machinery and affecting expression of oncoproteins such as survivin, c-myc, cyclin D1, VEGF, and HIF- $1 \alpha$. Alkaloid derivative 7-chloro-fascaplysin similarly inhibits cell survival through interference with the PI3K/Akt/mTOR pathway, which in turn modulates HIF-1 $\alpha$, eNOS and MMP-2/9 in the breast cancer cell line [62]. Experimental treatment of the HCT116 (colorectal), A375 (malignant melanoma), and H1975 (lung) xenografted tumor tissues resulted in decreased tumor angiogenesis and increased cleaved-caspase-3. Consequently, survivin and HIF- $1 \alpha$ are downregulated by suppressing 4EBP1-p70S6K1 axis-mediated de novo protein synthesis as was confirmed in the in vitro and in vivo experiments. In addition, fascaplysin inhibits vascular endothelial growth factor receptor 2 (VEGFR2) and tropomyosin-related kinase A (TRKA) via DFG-out non-competitive inhibition. These data suggest that fascaplysin inhibits TRKA and VEGFR2 and downregulates survivin and HIF-1 $\alpha$ resulting in suppression of tumor growth [58].

In our laboratory antitumor efficiency of fascaplysin and its synthetic derivatives such as 7-phenylfascaplysin, 3-chlorofascaplysin, 3-bromofascaplysin, and 10-bromofascaplysin in C6 glioma cells was compared in the in vitro studies. Fascaplysin applied in concentration range from 0.5 to $2 \mu \mathrm{M}$ exerted significant dose- and time-dependent antiproliferative and cytotoxic effects with IC50 about $1.0 \mu \mathrm{M}$ (Table 3). Inhibiting effects were noted to be associated with dose-dependent increase of the number of the glioma cells being in apoptosis stage. Inhibiting effect induced by fascaplysin in that model was significantly higher than that of temozolomide [63]. Cytotoxic influence of all fascaplysin derivatives investigated in our study was superior to the activity of unsubstituted fascaplysin. In particular, 3-bromofascaplysin and 7-phenylfascaplysin had shown the highest capacity to induce C6 glioma cell death [64].

Table 3. The structure and cytotoxic activity of fascaplisin against glioma C6 cells [64].

Compound

From the other hand, high cytotoxicity of fascaplysin regarding normal cells should be emphasized, which may be associated with its planar structure contributing d-s DNA intercalation [65]. Development of the regulating approaches for such unusual cytotoxicity requires further investigation of various fascaplysin derivative with better safety profile.

Carboline alkaloids (tricyclic pyridoindoles). Large group of natural, semisynthetic, and synthetic compounds is presented with tricyclic pyridoindoles, i.e., carbolines, which are classified as $\alpha$-carbolines (pyrido[2,3-b]indoles), $\beta$-carbolines (pyrido[3,4-b]indoles, $\gamma$ carbolines (pyrido[4,3-b]indoles) and $\delta$-carbolines (pyrido[3,2-b]indoles), depending on the position of the pyridine nitrogen relative to the indole. $\beta$-carbolines initially discovered in Peganum harmala and later widely found in medicinal plants and natural herbal products are the best investigated compounds [66]. Alkaloids composed of tricyclic moiety structurally relayed to both indole and carbazole compounds belong to the group of $\alpha$-carbolines. Among the marine species the representatives of the subtype Tunicata, 
mostly ascidians, are the source of $\alpha$-carboline alkaloids [67]. Natural $\alpha$-carbolines and their synthetic derivatives are of great interest as the lead compounds in the novel drug development. $\alpha$-Carboline related derivatives have been synthesized and experimentally demonstrated to show anticancer activities [68].

Six $\alpha$-carboline analogues were synthesized, designated as TJY-13, TJY-14, TJY-16, TJY-18, TJY-22, TJY-24, and then tested on the human glioma cell lines U87, U251, T98G and rat glioma C6. $48 \mathrm{~h}$ incubation of the glioma cells with those compounds resulted in inhibited proliferation as shown in Table 4. $\alpha$-Carboline analog TJY-16 (6-acetyl-9-(3,4,5trimethoxybenzyl)-9H-pyrido[2,3-b]indole) was found to be the strongest and highly potent inhibitor of the glioma cell viability applied in the nanomole concentration [69]. 50 $\mathrm{nm}$ concentration of TJY-16 induced cycle circle arrest in the phase G2/M of the U87 and T98G glioma cells. Moreover, in 24-48 h since the treatment of the tumor cells with TJY-16 the portion of the sub-G1 phase blocked cells was significantly greater. Microscopic images of the glioma cells treated with TJY-16 demonstrated apoptotic such as nuclear shrinkage and DNA condensation. Increased level of the cleaved caspase-3 was displayed with the use of western blot analysis. The activation of caspase- 8 and depolarization of mitochondrial membrane potential $(\Delta \Psi \mathrm{m})$ indicated that both extrinsic and intrinsic apoptotic pathways were involved in TJY-16-induced apoptosis. Surprisingly, cell death was noted in three human glioma cells but not observed in the C6 rat glioma cell culture.

TJY-16 administered intraperitoneally once per day for 10 days in a dose $24 \mathrm{mg} / \mathrm{kg}$ to the nude mice with xenograft tumor model of U87 glioma cells effectively inhibited tumor growth and induced caspase-3 activation. Anti-glioma effect of TJY-16 was significantly higher than that of temozolomide (orally administered once per day for 5 days in a dose $80 \mathrm{mg} / \mathrm{kg}$ [69]. Based on the results obtained authors of the research study considered $\alpha$ carboline derivative TJY-16 as a perspective agent for the therapy of malignant gliomas.

Table 4. Anti-glioma activities of $\alpha$-carboline derivatives against glioma cells in vitro [69].

\begin{tabular}{|c|c|c|c|c|c|}
\hline \multirow[t]{2}{*}{$\alpha$-Carboline derivative } & \multirow[t]{2}{*}{ Structure } & \multicolumn{4}{|c|}{$\mathrm{IC}_{50}(\mu \mathrm{M})$} \\
\hline & & U87 & T98G & U251 & C6 \\
\hline
\end{tabular}<smiles>[R]c1ccc2c(c1)c1cc([R])cnc1n2Cc1cc([R2])c([R4])c([R])c1</smiles>

\begin{tabular}{|c|c|c|c|c|c|}
\hline TJY-13 & $\mathrm{R}_{1}=\mathrm{H} ; \mathrm{R}_{2}=\mathrm{C}(=\mathrm{O}) \mathrm{CH}_{3} ; \mathrm{R}_{3}=\mathrm{H} ; \mathrm{R}_{4}=\mathrm{H} ; \mathrm{R}_{5}=\mathrm{H}$ & $>10$ & $>10$ & $>10$ & $>10$ \\
\hline TJY-14 & $\mathrm{R}_{1}=\mathrm{H} ; \mathrm{R}_{2}=\mathrm{C}(\mathrm{OH}) \mathrm{CH}_{3} ; \mathrm{R}_{3}=\mathrm{H} ; \mathrm{R}_{4}=\mathrm{H} ; \mathrm{R}_{5}=\mathrm{H}$ & $>10$ & $>10$ & $>10$ & $>10$ \\
\hline TJY-16 & $\mathrm{R}_{1}=\mathrm{H} ; \mathrm{R}_{2}=\mathrm{C}(=\mathrm{O}) \mathrm{CH}_{3} ; \mathrm{R}_{3}=\mathrm{OCH}_{3} ; \mathrm{R}_{4}=\mathrm{OCH}_{3} ; \mathrm{R}_{5}=\mathrm{OCH}_{3}$ & 0.042 & 0.043 & 0.088 & 0.050 \\
\hline TJY-18 & $\mathrm{R}_{1}=\mathrm{H} ; \mathrm{R}_{2}=\mathrm{H} ; \mathrm{R}_{3}=\mathrm{OCH}_{3} ; \mathrm{R}_{4}=\mathrm{OCH}_{3} ; \mathrm{R}_{5}=\mathrm{OCH}_{3}$ & $>10$ & $>10$ & $>10$ & $>10$ \\
\hline TJY-22 & $\mathrm{R}_{1}=\mathrm{CH}_{3} ; \mathrm{R}_{2}=\mathrm{H} ; \mathrm{R}_{3}=\mathrm{OCH}_{3} ; \mathrm{R}_{4}=\mathrm{OCH}_{3} ; \mathrm{R}_{5}=\mathrm{OCH}_{3}$ & $>10$ & 6.222 & 7.643 & 8.842 \\
\hline TJY-24 & $\mathrm{R}_{1}=\mathrm{H} ; \mathrm{R}_{2}=\mathrm{CH}_{3} ; \mathrm{R}_{3}=\mathrm{OCH}_{3} ; \mathrm{R}_{4}=\mathrm{OCH}_{3} ; \mathrm{R}_{5}=\mathrm{OCH}_{3}$ & 7.769 & 1.591 & 6.314 & 7.078 \\
\hline
\end{tabular}

Tetrahydroisoquinoline alkaloids. Tetrahydroisoquinoline alkaloids with antitumor activity isolated from marine species are commonly categorized into ecteinascidins and renieramycins. Ecteinascidin-743 (ET-743), which is also known as trabectedin, yondelis, and CID 108150 is one of the most potent representatives of ecteinascidins, isolated from Carribean ascidian (subphylum Tunicata) Ecteinascidia turbinata [70]. ET-743 was approved as a first line drug for the treatment of inoperable soft tissue sarcoma with high 
resistance to conventional chemotherapy. Mechanism of antitumor activity of the ET-743 is caused to its capacity to make a complex with minor groove of DNA double helix and alkylate N2 guanin thus preventing cell proliferation, DNA reparation, and activation of transcription. This cascade results in apoptosis of the target cells [71]. Three alkaloids namely ecteinascidin-770 (ET-770, a stabilized derivative of ET-743, isolated from ascidian E. thurstoni), a 2'-N-4"-pyridinecarbonyl derivative of ET-770 as well as renieramycin M, a major bis-1,2,3,4-tetrahydroisoquinolinequinone alkaloid from the marine sponge Xestospongia sp. were tested in human glioblastoma cells U373MG. All tested compounds exerted strong anti-glioma influence at the nanomolar concentrations by a 72 hour-treatment (Table 5) [72].

Table 5. Anti-glioblastoma activities of tetrahydroisoquinoline alkaloids against human glioblastoma cells U373MG [72].

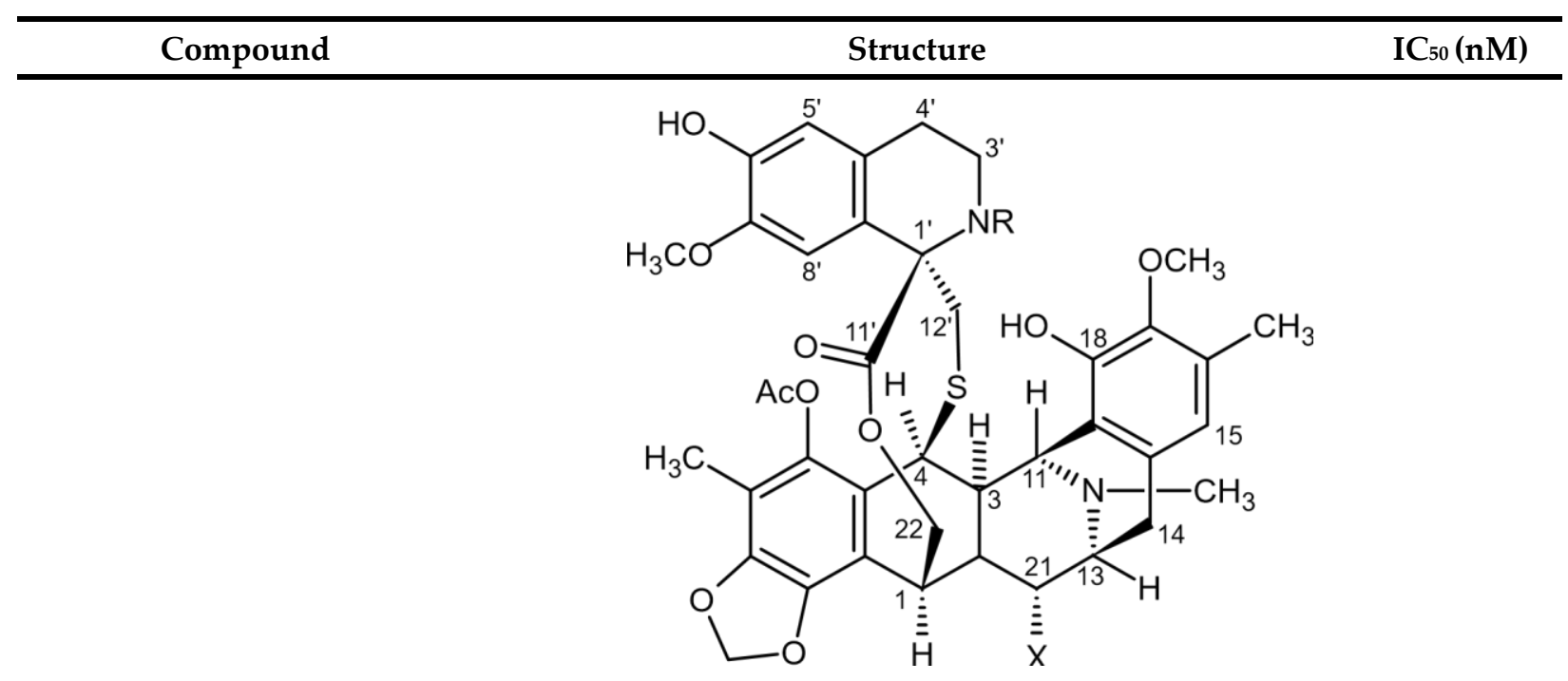

\begin{tabular}{lll}
\hline Ecteinascidin-770 & $\mathrm{R}=\mathrm{H}, \mathrm{X}=\mathrm{CN}$ \\
2'-N-4" -pyridinecarbonyl & & 4.83 \\
derivative of ET-770 & $\mathrm{R}$ \\
& $=$
\end{tabular}

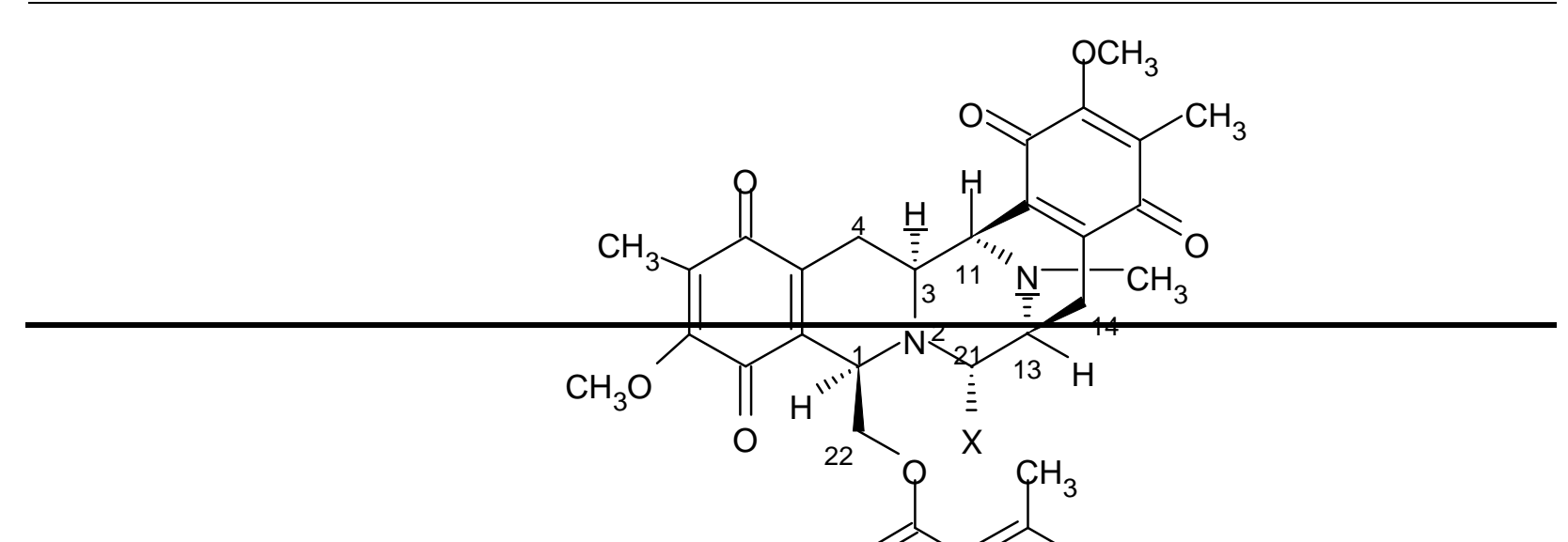


Influence regarding the U373MG cells exerted by each compound in IC50 concentration within $72 \mathrm{~h}$ resulted in induced PARP and CASP3 cleavage that reflect molecular markers of ongoing apoptosis. Investigation of the gene expression profile of the whole genome of the U373MG cells treated with alkaloids in IC50 concentrations within 24 hours have shown that ecteinascidin-770 reduced expression of 426 genes and upregulated 45 genes, renieramycin M suppressed expression of 274 genes and increased expression of 9 genes, and 2'-N-4"-pyridinecarbonyl derivative of ET-770 downregulated 417 genes and upregulated 84 genes. Generally, upregulated genes significantly prevailed over the genes with reduced expression for each tested compound. It should be noted that the set of 196 downregulated genes and 6 upregulated genes has shown the correspondence of tested compounds suggesting the presence of joint pathway involved in induction of apoptosis.

Analysis of molecular network made possible to identify ErbB (EGFR) signal pathway in the U373MG cells as a supplementation to the axonal and cell adhesion as significant down regulating gene pathways. ErbB (EGFR) signal pathway was found to be composed of FAK/PTK2, AKT3 и GSK3 $\beta$ acting as key molecules involved in cell migration and development of the nervous system. At the same time, a set of genes upregulated by alkaloid have shown significant linkage via cell cycle with CDC25A working as a hub molecule. It was also shown that suppressed expression of AKT3 by RNA interference reduce expression of the Bad phosphorylated form resulting in induced caspase-dependent apoptosis in the glioma cells [73]. AKT3 is known to be required for anchorage-independent growth of human glioma cells. Glycosynthase kinase $3 \beta$ (GSK3 $\beta$ ) involved in apoptotic pathways presents a serine/threonine kinase that regulates in an integrated manner Wnt/ $\beta$-catenin, Hedgehog, and receptor tyrosine kinase (RTK) signaling pathways playing key role in such cellular functions as glycogen metabolism, cell differentiation, proliferation, and apoptosis. Downregulating effects of siRNA towards GSK3 $\beta$ activity inhibits cell migration and induces apoptosis of glioma cells via c-Myc activation and suppression of the nuclear factor- $\kappa \mathrm{B}$ (NF- $\mathrm{KB}$ ) activity [74]. The cell cycle progression inhibiting and activating processes are known to be regulated by the complex checkpoint mechanism that includes cyclins A, B, D, and E along with cyclindependent kinases (CDKs) and CDK inhibitors (CDKIs) of both the Cip/Kip and Ink4 families. The hypophosphorylated $\mathrm{Rb}$ protein interacts with the E2F family transcription factors E2F1, E2F2, and E2F3, and activates gene expression that is crucial for the cell cycle progression. $\mathrm{Rb}$ protein, hyperphosphorylated by cyclin D1-CDK4 and cyclin E1-CDK2 complexes, in turn releases E2Fs and represses the cell cycle gene expression [75]. Thus, Rb/E2F pathway acts a molecular switch contributing to either progressing or arrest of the cell cycle. The U373MG molecular network in the glioma cells composed of the integral set of downregulated and upregulated genes generally affected by all tested tetrahydroisoquinoline alkaloids was found to have a significant relation with transcription regulating mechanisms via transcription factors $\mathrm{Rb} / \mathrm{E} 2 \mathrm{~F}$ [72]. These findings suggest that those alkaloids work as the DNA-alkylating agents interfering cell division finally resulting in apoptosis of the target cells.

It is already known that the human glioblastoma cell lines MO59K and MO59J are basically characterized by the presence or absence of the DNA-dependent protein kinase (DNA-PK) catalytic subunit, respectively, which considered a part of the DNA double- 
strand-break repair pathway. These cells have shown different response to the treatment with ET-743. MO59J cells were much more sensitive to ET-743 treatment compared to the MO59K cells with a 5-fold lower values of ET-743 IC50 values $(0.041 \pm 0.004$ vs. $0.2 \pm 0.02$ $\mathrm{nM})(\mathrm{p}<0.05)$ [76]. These results confirm that tetrahydroisoquinoline alkaloids possess a unique mechanism of interaction with DNA.

Pyrrole alkaloids. Marine pyrrole-derived alkaloids have been intensively investigated in numerous studies focused on elucidation of their structure and biological activities. Alkaloids rigidins A, B, C, D, and E initially isolated from the tunicate Eudistoma cf. Rigida $[77,78]$ and later synthesized [79] were the most notable ones among the group of pyrrole alkaloids but have shown low activity regarding cultivated human cancer cells. Further investigations of synthetic compounds led to development of the promising approaches to modification of the 7-deazaxanthine skeleton that typical of rigidins converting into corresponding 7-deazahypoxanthines (Figure 4).<smiles>[X]c1cc(C(=O)c2[nH]c3[nH]c(=O)[nH]c(=O)c3c2-c2ccc(O)c([X])c2)ccc1O</smiles>

Rigidin A $\quad \mathrm{X}=\mathrm{H}, \mathrm{Y}=\mathrm{H}$

Rigidin B $\mathrm{X}=\mathrm{OMe}, \mathrm{Y}=\mathrm{H}$

Rigidin $\mathrm{C} \quad \mathrm{X}=\mathrm{H}, \mathrm{Y}=\mathrm{OMe}$

Rigidin D $\quad \mathrm{X}=\mathrm{OMe}, \mathrm{Y}=\mathrm{OMe}$

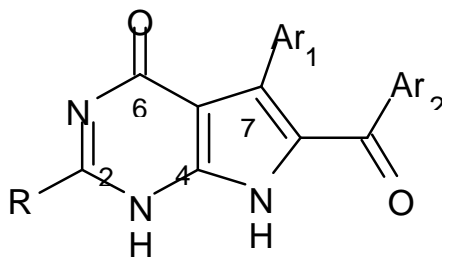

C2-aryl- and C2-alkyl-7-deazahypoxanthines

Figure 4. Structures of the marine alkaloid rigidins A, B, C, D, and their 7-deazahypoxanthine based synthetic analogues [79].

Novel marine rigidin analogues C2-aryl- and C2-alkyl-7-deazahypoxanthines obtained via synthesis techniques purposed for construction of the pyrrolo[2,3-d]pyrimidine ring system were applied in submicromolar and nanomolar quantities and exerted strong anti-proliferating activity against different cell lines including multidrug resistant tumors such as glioblastoma, melanoma, and non-small cell lung cancer. As the only difference between 7-deazaxantine rigidin scaffold and 7-deazahypoxanthines skeleton structure is the lack of carbonyl group at $\mathrm{C} 2$ in the latter structure, such modifications this position are supposed to be critical for their activity. It was demonstrated that one of the C2-methyl-7-deazahypoxanthines namely 6-benzoyl-2-methyl-5-phenyl-1H-pyrrolo[2,3d]pyrimidin- $4(7 \mathrm{H})$-one, exerted greatest antiproliferative activity against human glioblastoma cell line U-87 (GI50 = 0.077 $\pm 0.002 \mu \mathrm{M})$, human cervical adenocarcinoma HeLa (GI50 $=0.029 \pm 0.001 \mu \mathrm{M})$, breast adenocarcinoma MCF-7 $(0.035 \pm 0.003 \mu \mathrm{M})$, and lung carcinoma cell line A549 $(0.25 \pm 0.01 \mu \mathrm{M})$. Other synthetic C2-methyl-7-deazahypoxanthines also exerted substantial antiproliferative effects against $\mathrm{U}-87$ cell line with a concentration range from $0.90 \pm 0.16 \mu \mathrm{M}$ to $9.23 \pm 2.13 \mu \mathrm{M}$ being inferior to the original C7-phenyl analogue but still preserving submicromolar potency, except only one derivative demonstrating micromolar potency (Table 6). The drop of the activity of this analogue could be also explained by its polar character impairing cell membrane permeability. 
Synthetic 7-deazahypoxanthines were shown to be able to disrupt microtubule cytoskeleton organization in the tumor cells by binding to the colchicine site of $\beta$-tubulin [79]. In the cultivating HeLa cell line one of the 7-deazahypoxanthines at concentrations between 1 and $2 \mu \mathrm{M}$ induced significant alterations of the mitotic microtubule organization. The interphase microtubules were less affected at these concentration range suggesting that this compound was primarily affecting dynamic microtubules. It exerted slight influence on the stable interphase or spindle microtubules but at the same time induced a marked mitotic spindle shift that may be related to the astral microtubule defects. Astral microtubules as the most dynamic microtubule population at mitotic stage are likely to be the most sensitive targets for tubulin-targeting drugs such as 7-deazahypoxanthines [79].

Table 6. The structure and antiproliferative activities of C2-methyl-7-deazahypoxanthines against U-87 glioma cells [79].

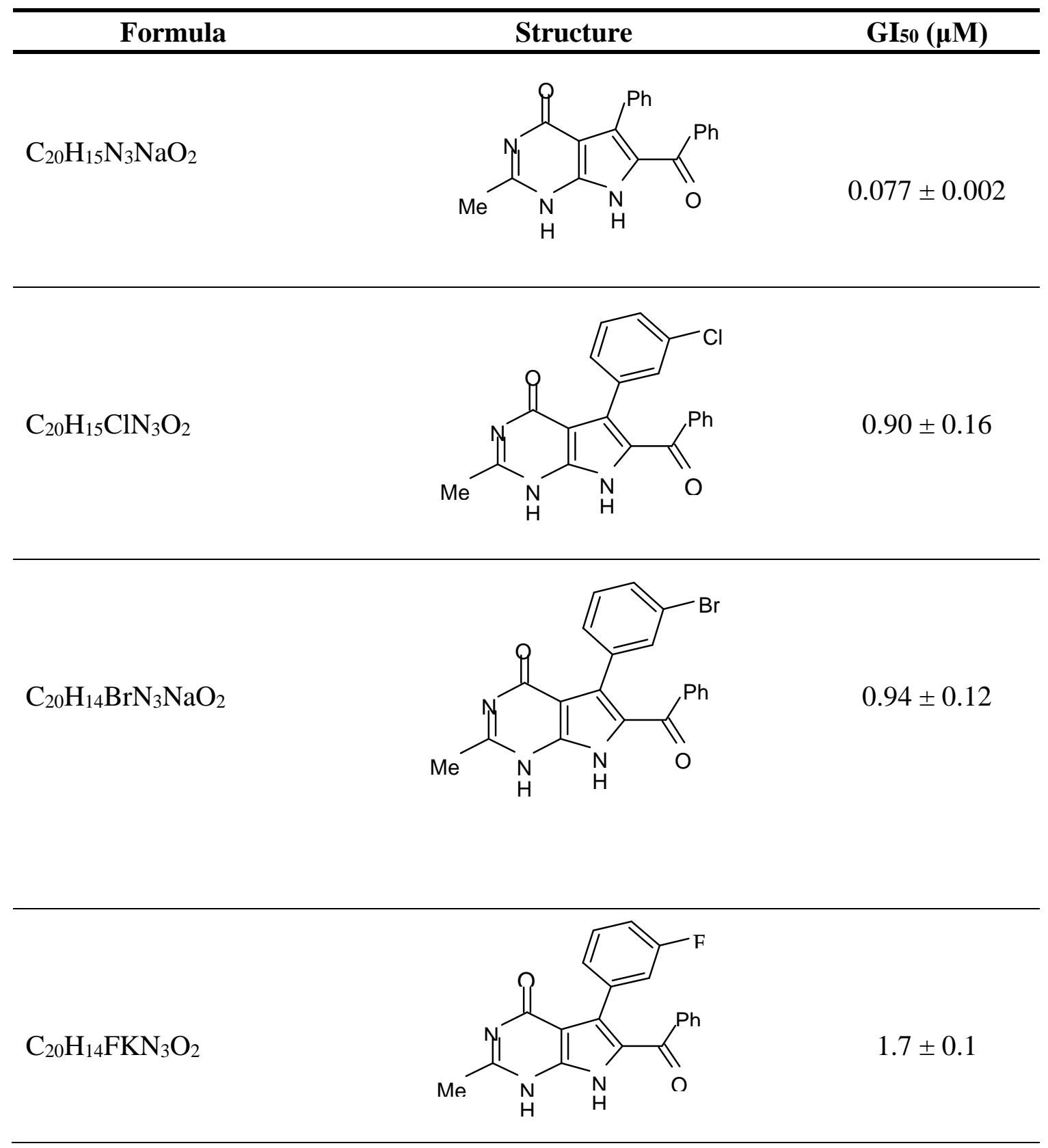


$\mathrm{C}_{19} \mathrm{H}_{14} \mathrm{~N}_{4} \mathrm{NaO}_{2}$<smiles>Cc1nc(=O)c2c(-c3cccnc3)c(C(=O)c3ccccc3)[nH]c2[nH]1</smiles>

$\mathrm{C}_{19} \mathrm{H}_{14} \mathrm{BrN}_{4} \mathrm{O}_{2}$<smiles>Cc1nc(=O)c2c(-c3cncc(Br)c3)c(C(=O)c3ccccc3)[nH]c2[nH]1</smiles>

$\mathrm{C}_{20} \mathrm{H}_{14} \mathrm{Br}_{2} \mathrm{~N}_{3} \mathrm{O}_{2}$<smiles>Cc1nc(=O)c2c(-c3cc(Br)cc(Br)c3)c(C(=O)c3ccccc3)[nH]c2[nH]1</smiles>

Pyrrospirone alkaloids. Novel pyrrospirone alkaloids C, D, E, F, G, H and J as well as penicipyrroether A were isolated from marine fungus Penicillium sp. (ZZ380 strains) generally found in the wild sea crab Pachygrapsus crassipes. These alkaloids along with other chemically related compounds make a family of fungal secondary metabolites, which are also called hirsutellones and contain a unique 13-membered ether ring composed of specific structural units such as decahydrofluorene, para-cyclophane and pyrrolidinone. That fungal metabolite family isolated from fungi belongs to the genera Cylindrocarpon, Embellisia, Hirsutella, Lewia, Neonectria, Penicillium and Trichoderma and divided into four chemical groups: hirsutellones, pyrrospirones, pyrrocidines, and GKK1032s [80]. Some of these metabolites were proved in experiments with the cancer cell line to have cytotoxic activity as well as exert antifungal and antibacterial effects.

Structures of the pyrrospirone alkaloids were deciphered by extensive NMR spectroscopic analyses, HRESIMS data, and Mosher's method (Figure 5). Pyrrospirones $C$ and $\mathrm{D}$ have the same molecular formula of $\mathrm{C} 33 \mathrm{H} 43 \mathrm{NO}$. From the structural point of view both of them are composed of two carbonyls, six aromatic carbons, two olefin carbons, four quaternary carbons, two oxymethines, one methoxyl, six methines, five methylenes and five methyls as well as these compounds have a close spiro ring system but different configurations at $\mathrm{C}-17$. In the similar fashion pyrrospirones $\mathrm{E}$ and $\mathrm{F}$ have the same molecular formula of $\mathrm{C} 32 \mathrm{H} 41 \mathrm{NO}$. The 13C NMR data of pyrrospirones $\mathrm{E}$ and $\mathrm{F}$ show close similarities with those of pyrrospirones $C$ and $D$, respectively, except the different chemical shifts for C-19 to C-21 due to the absence of a methoxy at C-19 in pyrrospirones $E$ and $\mathrm{F}$. This indicates pyrrospirones $\mathrm{E}$ and $\mathrm{F}$ are the analogues of pyrrospirones $\mathrm{C}$ and $\mathrm{D}$ without methoxyl at C-19. Similar to pyrrospirones $C$ and $D$, the structural difference between pyrrospirones $\mathrm{E}$ and $\mathrm{F}$ is caused by different $\mathrm{C}-17$ configurations. Molecular formula of pyrrospirones $\mathrm{G}$ is C32H39NO5, which is two protons less than that of pyrrospirones E. Structural difference between pyrrospirones $\mathrm{G}$ and $\mathrm{E}$ is presented with oxymethine at $\mathrm{C}$ 17 in $\mathrm{E}$ is replaced by a carbonyl group in G compound. Pyrrospirone H molecular formula is $\mathrm{C} 32 \mathrm{H} 41 \mathrm{NO} 4$. That is 16 mass units less than that of pyrrospirone E. Pyrrospirone $\mathrm{H}$ is considered a pyrrospirone $\mathrm{E}$ analogue with a methylene attached at $\mathrm{C}-17$. The molecular formula pyrrospirone I is $\mathrm{C} 32 \mathrm{H} 39 \mathrm{NO} 4$, which is two protons less than that of 
pyrrospirone $\mathrm{H}$. The pyrrospirone I structure is considered as analogue of pyrrospirone $\mathrm{H}$ with a double bond at C-16 and C-17 [80]. Penicipyrroether A has a molecular formula $\mathrm{C} 30 \mathrm{H} 37 \mathrm{NO} 5$ whereas $\mathrm{C} 32 \mathrm{H} 41 \mathrm{NO} 6$ is a molecular formula pyrrospirone J. Despite structural parts of rings $\mathrm{A}-\mathrm{C}, \mathrm{F}$ and $\mathrm{G}$ for penicipyrroether $\mathrm{A}$ and pyrrospirones $\mathrm{C}-\mathrm{I}$ are the same, the structures of penicipyrroether $\mathrm{A}$ and pyrrospirones have specific differences. First of all, D ring contains five-membered ether ring for penicipyrroether A and cyclohexanone for pyrrospirones. Then, penicipyrroether A does not have spiro junction for rings D and E. NMR spectroscopic data analysis and HRESIMS data (high resolution electrospray ionization mass spectroscopy) demonstrated that compound pyrrospirone $\mathrm{J}$ is an analogue of penicipyrroether A with the same structural part of rings A, B, F and G but possesses an epoxy moiety at C-10 and C-11 and a different five-membered ether ring D fused with ring $\mathrm{E}$ through a spiro carbon of $\mathrm{C}-15$. In addition, the dehydro-pyrrolidinone moiety (ring E) in penicipyrroether $\mathrm{A}$ is replaced by a pyrrolidinone moiety in pyrrospirone $\mathrm{J}[81]$.

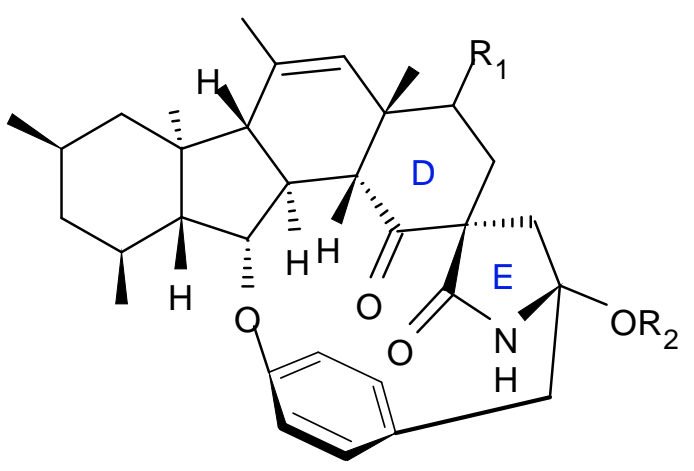

Pyrrospirone $\mathrm{C}: \mathrm{R}_{1}=\alpha \mathrm{OH}, \mathrm{R}_{2}=\mathrm{CH}_{3}$

Pyrrospirone D: $\mathrm{R}_{1}=\beta \mathrm{OH}, \mathrm{R}_{2}=\mathrm{CH}_{3}$

Pyrrospirone E: $\mathrm{R}_{1}=\alpha \mathrm{OH}, \mathrm{R}_{2}=\mathrm{H}$

Pyrrospirone $\mathrm{F}: \mathrm{R}_{1}=\beta \mathrm{OH}, \mathrm{R}_{2}=\mathrm{H}$

Pyrrospirone G: $\mathrm{R}_{1}=\mathrm{O}, \mathrm{R}_{2}=\mathrm{H}$

Pyrrospirone $\mathrm{H}$ : $\mathrm{R}_{1}=\mathrm{H}, \mathrm{R}_{2}=\mathrm{H}$
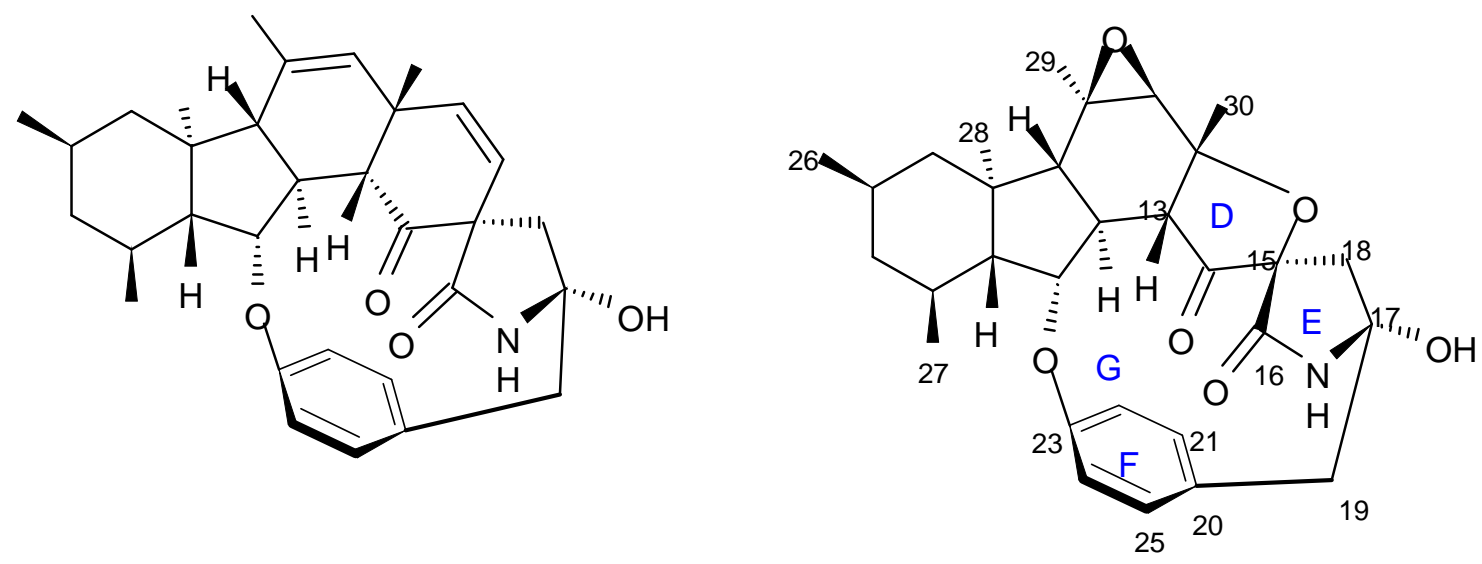

Pyrrospirone I

Pyrrospirone J

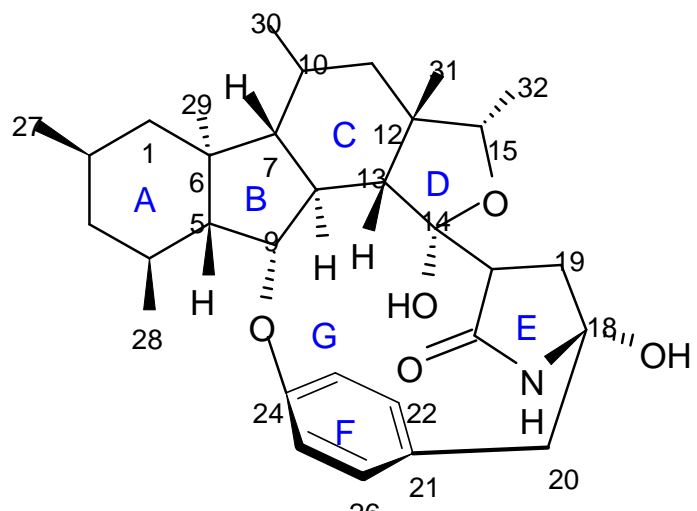


Penicipyrroether A

Figure 5. The structure of pyrrospirones $\mathrm{C}-\mathrm{J}$ and penicipyrroether $\mathrm{A}$ isolated from the cultures of marine-derived fungus Penicillium sp. ZZ380 [81].

Inhibiting activity regarding proliferation of the glioma cells U87MG, U251, SHG44 and $\mathrm{C} 6$ of the pyrrospirone alkaloids isolated from the ZZ380 strain were investigated. The results showed that pyrrospirone $\mathrm{G}$ exerts relatively strong activity regarding aforementioned cell lines with IC50 values approximately between 1.06 and $8.52 \mu \mathrm{M}$. Pyrrospirones $\mathrm{C}, \mathrm{D}, \mathrm{E}, \mathrm{F}, \mathrm{H}, \mathrm{I}$, and J have demonstrated moderate anti-glioma activity in IC50 values generally between 7.44 and $26.64 \mu \mathrm{M}$ (Table 7) [80]. Penicipyrroether A exerted strong inhibiting influence on the cell proliferation in glioma lines U87MG and U251 with the IC50 values 1.64 and $5.50 \mu \mathrm{M}$, relatively [81]. Doxorubicin used as a positive control have suppressed glioma cell proliferation with the IC50 values approximately 1.20 and 8.03 $\mu \mathrm{M}$, respectively. These results suggest that antiglioma activity of penicipyrroether $\mathrm{A}$ is equivalent or slightly stronger than doxorubicin potency. Furthermore, cytotoxicity of penicipyrroether A and doxorubicin against normal human astrocytes (HA, Cat. No. 1800, ScienCell), expressed in CC50 was shown to be $23.28 \pm 1.05 \mu \mathrm{M}$ and $8.57 \pm 0.16 \mu \mathrm{M}$, respectively. Selectivity index (CC50/IC50) of penicipyrroether A towards U87MG and U251 cells was found to be 14.2 and 4.2, respectively, which substantially higher than that of doxorubicin i.e. 7.1 and 1.1, respectively [81].

Penicipyrrodiether A containing phenol A derivative fused to the pyrrolidinone core via the five membered ether ring added have shown moderate antiglioma activity manifesting in reduced proliferation of the human glioma U87-MG, U251, SHG-44 cells, and rat glioma C6 cells with IC50 values between 11.32 and $29.10 \mu \mathrm{M}$. Doxorubicin used as positive control was found to exert its activity with IC50 values of 0.70 to $9.61 \mu \mathrm{M}[80]$.

Table 7. Anti-glioma activity of pyrrospirones C-J and penicipyrroether A isolated from the cultures of marine-derived fungus Penicillium sp. ZZ380 against the proliferation of glioma cells [80,81].

\begin{tabular}{lcccc}
\hline \multicolumn{1}{c}{ Compound } & \multicolumn{4}{c}{ IC $50(\mu \mathrm{M})$} \\
\cline { 2 - 5 } & U87MG & U251 & SHG44 & C6 \\
\hline Pyrrospirone C & $12.15 \pm 0.61$ & $22.12 \pm 1.11$ & $10.03 \pm 0.10$ & $13.87 \pm 1.26$ \\
\hline Pyrrospirone D & $9.95 \pm 0.50$ & $23.39 \pm 1.27$ & $13.74 \pm 0.55$ & $14.56 \pm 0.95$ \\
\hline Pyrrospirone E & $16.24 \pm 0.68$ & $26.64 \pm 2.14$ & $15.76 \pm 1.20$ & $21.03 \pm 3.15$ \\
\hline Pyrrospirone F & $12.44 \pm 0.81$ & $22.82 \pm 1.15$ & $8.93 \pm 0.76$ & $14.87 \pm 1.66$ \\
\hline Pyrrospirone G & $\mathbf{1 . 0 6} \pm \mathbf{0 . 0 5}$ & $\mathbf{1 . 2 8} \pm \mathbf{0 . 0 6}$ & $\mathbf{2 . 1 4} \pm \mathbf{0 . 1 1}$ & $\mathbf{8 . 5 2} \pm \mathbf{1 . 0 1}$ \\
\hline Pyrrospirone H & $12.89 \pm 0.64$ & $23.92 \pm 1.20$ & $13.02 \pm 0.79$ & $23.24 \pm 2.24$ \\
\hline Pyrrospirone I & $13.67 \pm 0.53$ & $13.46 \pm 0.76$ & $7.44 \pm 0.97$ & $19.18 \pm 2.11$ \\
\hline Pyrrospirone J & $10.52 \pm 0.62$ & $17.92 \pm 0.93$ & n.d.* & n.d. \\
\hline Penicipyrroether A & $\mathbf{1 . 6 4 \pm 0 . 0 5}$ & $\mathbf{5 . 5 0 \pm 0 . 1 2}$ & n.d. & n.d. \\
\hline Doxorubicin & $1.20 \pm 0.06$ & $8.03 \pm 1.20$ & $0.67 \pm 0.10$ & $0.47 \pm 0.10$ \\
\hline
\end{tabular}

\footnotetext{
${ }^{*}$, no data.
} 
Therefore, among the pyrrospirone alkaloids that have been studied, at least two compounds namely pyrrospirone $\mathrm{G}$ and penicipyrroether $\mathrm{A}$ are worth attention as promising antiglioblastoma agents.

Pyrrocidine alkaloid. Novel pyrrocidine alkaloid trichobamide A isolated from the ascidian-derived fungus Trichobotrys effuse 4729 had shown significant inhibition of the cell proliferation of two glioma lines such as U251 and SNB19. This novel alkaloid (molecular formula $\mathrm{C} 35 \mathrm{H} 39 \mathrm{NO} 5$ ) is different due to the presence of unprecedented tetrahydro-5H-furo[2,3-b]pyrrol-5-one moiety (Figure 6). Structurally, trichobamide A represents the first example of tetrahydro-5H-furo[2,3-b]pyrrol-5-one ring system and biosynthetically is related to a family of pyrrocidine alkaloids bearing a macrocyclic ether and succinimide-derived moieties.

Within the concentration range from 1 to $10 \mu \mathrm{M}$ trichobamide A exerted concentration- and time-dependent inhibiting effects against glioma cells. It was found out that the mechanism of antiproliferative activity of trichobamide A is directly related with increased relative expression of proteins P53, Bax, caspase 3, and caspase 9 as well as reduced expression of Bcl-2 in glioma cells. Trichobamide A was discussed to induce apoptosis in human glioma cells through a pathway upregulated by P53/Bax/Bcl-2 [82].

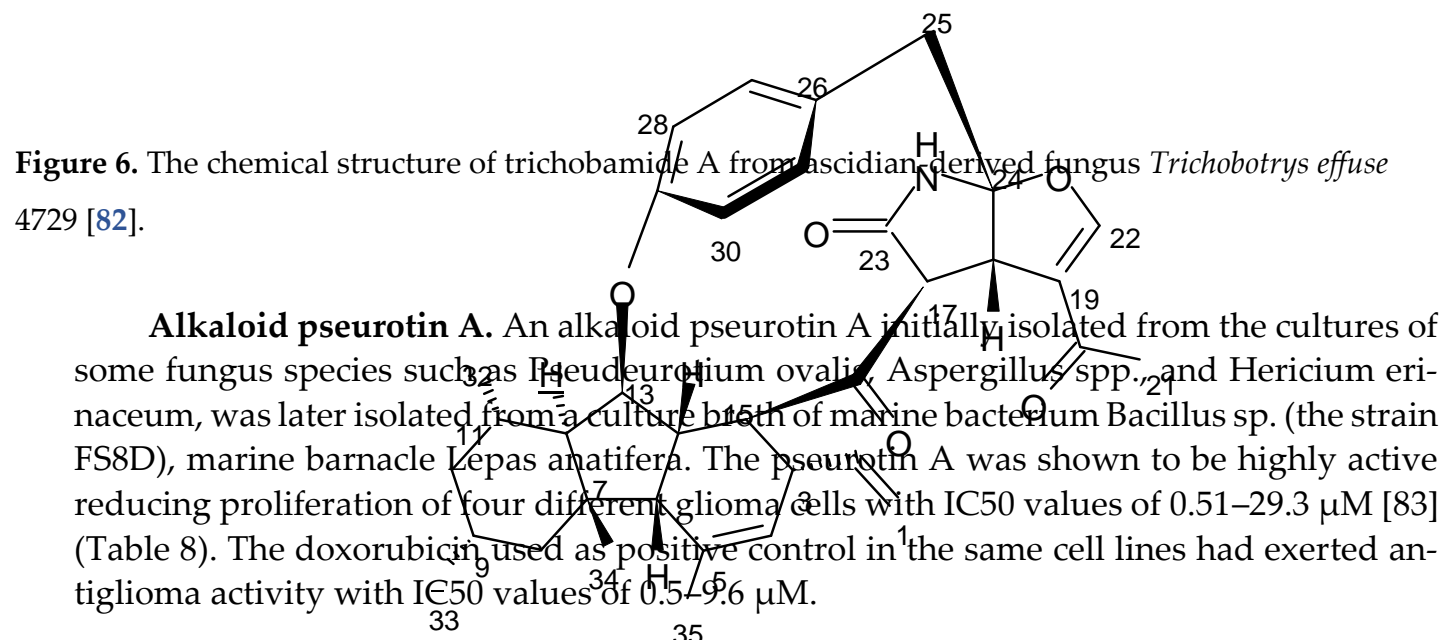
35

Table 8. The structure and anti-glioma activity of pseurotin A isolated from marine bacterium Bacillus sp. FS8D [83].

\begin{tabular}{lccccc}
\hline Compound & Structure & \multicolumn{4}{c}{$\mathrm{IC}_{50}(\mu \mathrm{M})$} \\
\cline { 2 - 5 } & & $\mathrm{C} 6$ & $\mathrm{U} 251$ & SHG-44 & U87-MG \\
\hline
\end{tabular}




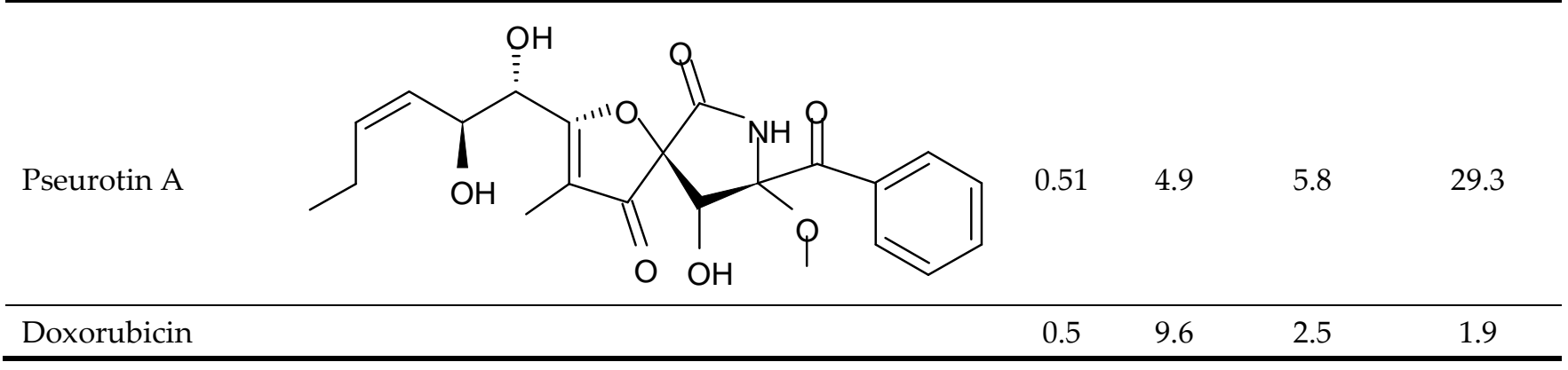

Mechanisms of antitumor activity were investigated in the U87-MG cell line. Pseurotin A was shown to reduce the expression levels of some pyruvate kinases M2 (PKM2), especially lactate dehydrogenase 5 (LDH5), which is a key enzyme responsible for accelerating conversion of pyruvate to lactate, the final product of tumor glycolysis. In addition, pseurotin A upregulates expression of the pyruvate dehydrogenase beta (PDHB), adenosine triphosphate synthase beta (ATPB) and cytochrome C (Cyto-C) [83], which are main enzyme components in the processes of tricarboxylic acid (TCA) cycle and oxidative phosphorylation in normal cells.

Based in the results obtained through the study, authors have proposed that pseurotin A exerts anti-glioma effects via inhibition of the accelerated rate of glycolysis int the glioma cells through the downregulation of PKM2 and LDH5 expression. Also it alters tumor metabolic pathway to the process of oxidative phosphorylation by enhancing TCA cycle and oxidative phosphorylation activities through the upregulation of PDHB, ATPB and Cyto-C.

Polycyclic diamine alkaloids. Polycyclic diamine alkaloids represent the specific class of alkaloid compounds that are mostly composed ammonia, propenal, and longchain dialdehydes as the universal building blocks. These alkaloids are typically found in the marine sponges belonging to the order Haplosclerida with the major part of species are made of representative of four families of that order (Callyspongiidae, Chalinidae, Niphatidae, and Petrosiidae). Two recently described alkaloids namely papuamine and haliclonadiamine as well as two newly discovered compounds called neopetrocyclamine A and neopetrocyclamine B were isolated from the Indonesian sponge Neopetrosia cf exigua and then screen for their efficacy in vitro against human glioblastoma cell line SF295. A symmetrical diamine compound papuamine was originally discovered from a marine sponge belonging to the genus Haliclona from Papua-New Guinea [84] and from Palau [85]. In the Palau specimens, papuamine was just a minor component in its mixture with haliclonadiamine, which is an unsymmetrical diastereoisomer of papuamine.

Neither neopetrocyclamine A nor neopetrocyclamine B had shown significant cytotoxicity at $20 \mu \mathrm{M}$. Nevertheless, both alkaloids papuamine and haliclonadiamine demonstrated strong inhibiting influence against glioblastoma cell (Table 9). As it is shown on the table, papuamine is more potent than haliclonadiamine against glioblastoma SF-295 cells because its GI50 value is almost 8-fold lower [86]. Author suggested that the stereogenic center at C-6 of the papuamine play important role in the anti-glioma activity.

Papuamine was recently shown to possess antimetastatic activity against MDA-MB231 breast cancer cells and reduce tumor cell vitality via mitochondria damage and JNK activation [87]. However, specific molecular targets and mechanisms of the anti-glioma effects exerted by these compounds are to studied yet

Polycyclic granulatimide alkaloids. Alkaloids granulatimide and isogranulatimide isolated from ascidian Didemnum granulatum were shown to exert inhibiting activity against checkpoint kinase 1 (Chk1), which is a Ser/Thr protein kinase and a key protein in the DNA damage response. As it was described before Chk1 is considered as an emerging target for anticancer therapeutics, whereas Chk1 inhibitors themselves are thought to be potential antitumor agents acting through enhancing activity of DNA damaging agents such as standard cytotoxic or radiation therapy. Both isogranulatimide and granulatimide selectively inhibited the G2 cell cycle checkpoint of mammalian cancer cells whereas the 
normal cells were affected to a much smaller extent. Therefore, the granulatimide alkaloids are considered as a novel class of potentially useful compounds for cancer treatment [88].

Table 9. The structure and cytotoxicity activity of polycyclic diamine alkaloids from marine sponge Neopetrosia cf exigua against glioblastoma SF-295 cells [86].

Compound Structure

Haliclonadiamine

Neopetrocyclamine A

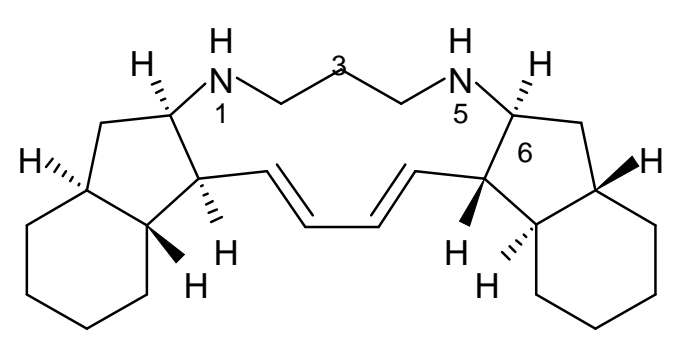

6.3

Neopetrocyclamine B
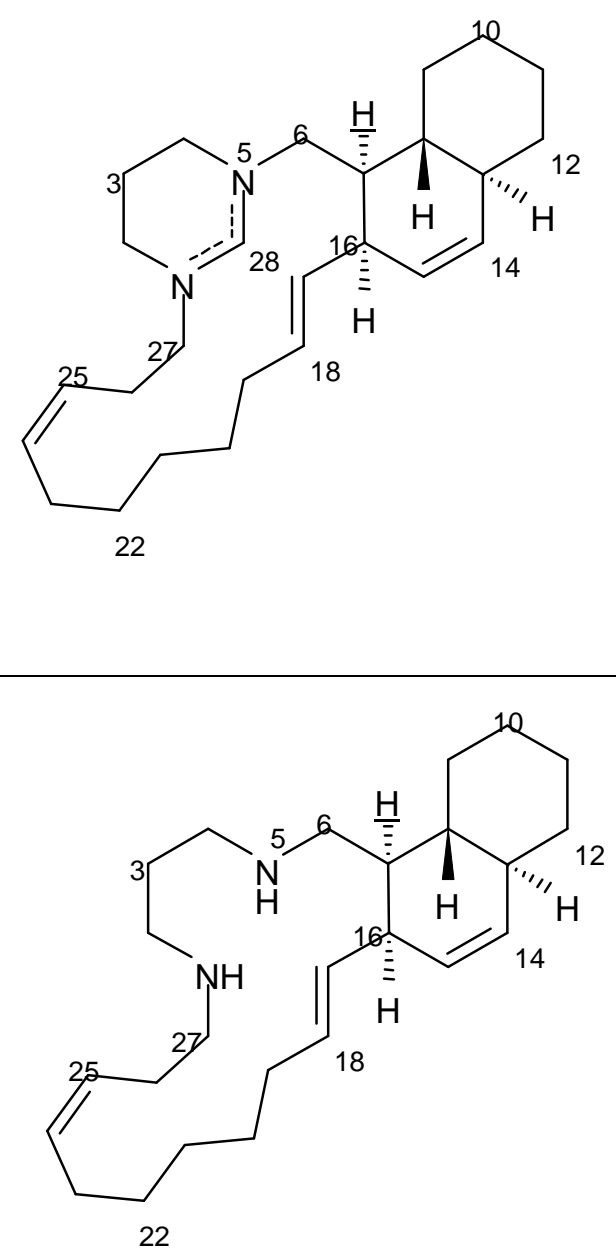
The result of the molecular docking study suggested that amino group at the paraindolic position in the granulatimide framework could be directed towards the binding site opening. After that, 17 amido and amino analogues of granulatimide $и$ isogranulatimide were tested in the oligodendroglioma Hs683 and glioblastoma U373 cells and the results demonstrated that two derivatives with open structure exerted antiglioma activity exceeding the one of the. Granulatimide. Their anticancer effects were noted at micromolar concentrations (Table 10) [89]. It is worth noting that the closure of the open structure into the corresponding final product usually resulted in the lowered in vitro inhibition of tumor growth. Nevertheless, no relevant inhibition of Chk1 was detectable with these structures [89]. That may suggest the presence of other pathways playing important role in the inhibition of the glioma cell proliferation.

Table 10. In vitro inhibitory concentrations of granulatimide, isogranulatimide from ascidian Didemnum granulatum and amino analogues against glioma cell lines [89].

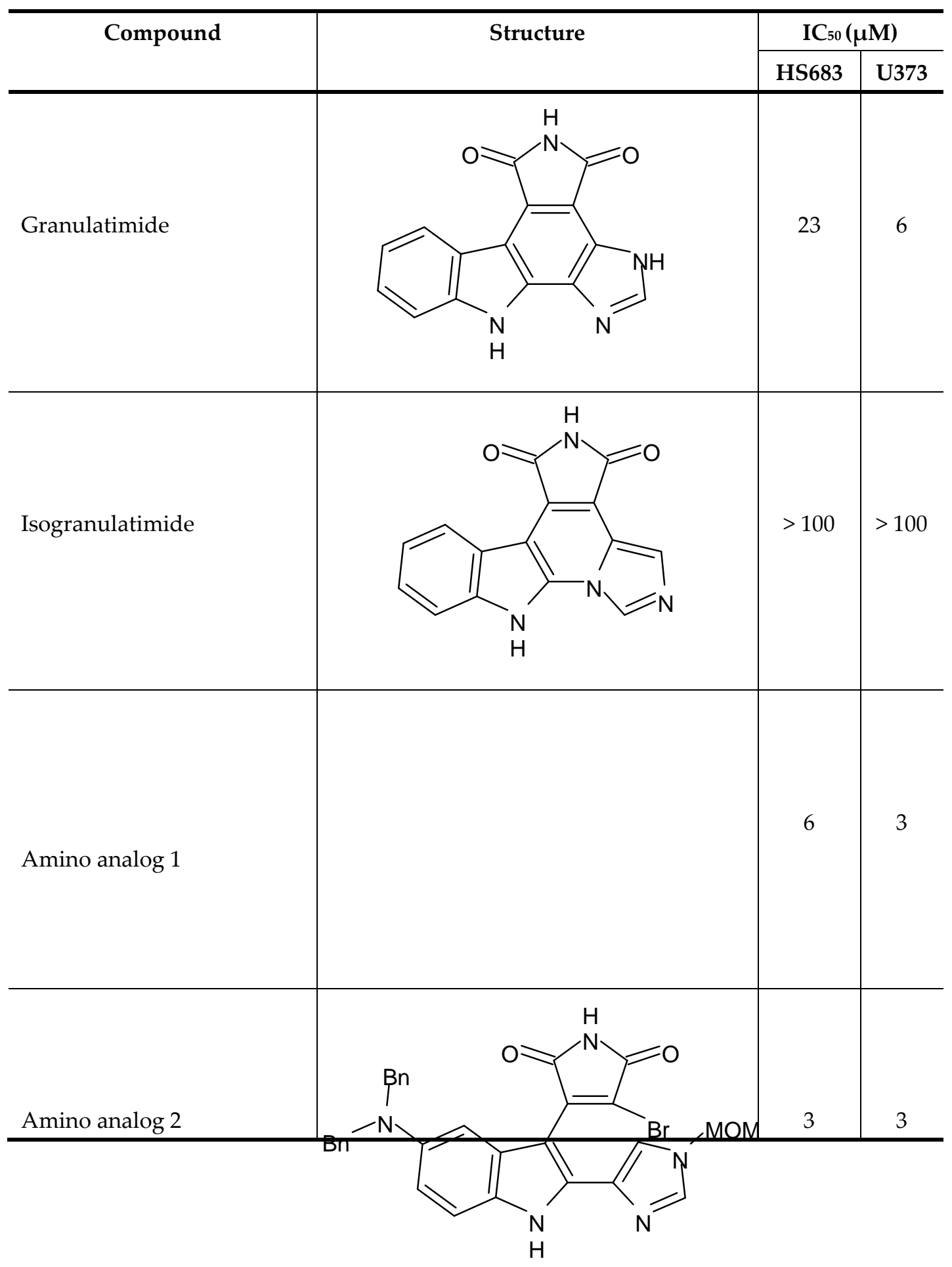




\section{Antitumor antibiotics}

Antibiotics (antimicrobials) presents a large diverse group of chemical compounds isolated from natural sources (microorganisms, plants, and animals) or obtained via semisynthetic or synthetic technologies exerting suppressing or killing effects on any microorganisms (bacteria, fungi, viruses, and parasites). Antibiotics may have cytotoxic or cytostatic activity against bacteria helping immune system of the body counteract infectious agents. They may act via various mechanisms of action that include protein synthesis inhibition and suppressing synthesis of the membrane and cell wall components, inhibition of the DNA replication, inhibition of the RNA transcription, or direct destruction of the cell membrane. Main sources of the marine-derived antimicrobial agents are bacteria (including bacteria from marine sediments and marine water samples, marine alga-associated and marine/mangrove plant-associated bacteria, and invertebrate-associated bacteria), fungi (including fungi from marine sediments and marine water samples, marine alga-, plant- and invertebrate-associated fungi), algae, and invertebrates (mainly, marine sponges and ascidians) [90].

In addition to their antimicrobial activity, some antibiotics provide antiproliferative influence playing valuable role in chemotherapy of malignant tumors. Therefore, they are commonly termed antitumor antibiotics. Among the antitumor antibiotics approved for clinical practice today, the most commonly used drug include anthracyclines (daunorubicin, aclarubicin, idarubicin, amrubicin), glycoprotein antibiotic bleomycin, chromoprotein actinomycin D, mithramycin belonging to the aureolic acid antibiotic group, mitoxantrone from the group of mitomycins, semisynthetic epothilone B derivative ixabepilone, and rapamycin derivative everolimus [91]. In the present review section, we will give our attention to antibiotics with antiglioma activity.

Actinomycins. Actinomycins present a family of closely related chromopeptides exerting both anticancer and antibiotic activities and obtained from a variety of streptomyces strains. Each strain synthesizes a unique set of actinomycin compounds. All actinomycins consist of 2-amino-4,6-dimethyl-3-oxo-phenoxazine-1,9-dicarboxylic chromophore with two pentapeptide lactone rings separately connected to one of the carboxylic groups in positions 1 and 9 in the phenoxazinone core. Variations in the actinomycin structure are possible but restricted to amino acid substitutions in the peptide lactone rings. Phenoxazinone core is unmodified as it si essential for their activity. In some cases, cyclization between the amino group in position 2 of the chromophore with functional groups of modified threonine residues in the $\beta$-ring, such as chlorine or a hydroxyl group at position 4 of the threonine component in the $\beta$-ring can be observed.

Up to date, about 50 actinomycins have been isolated from various species of Streptomyces including actinomycin D, N-demethylactinomycins, actinomycins C, F, Z, G and Y. Some actinomycin analogues, such as methylated actinomycin D and actinomycin $Z$ analogue having additional oxygen bridge between chromophore and $\beta$-depsipentapeptide as well as actinomycins D1-D4 and neo-actinomycins A and B, contain structurally modified cyclopeptide rings or chromophore [92]. Actinomycins were proved to provide excellent antitumor activity and thus found common application in the antitumor therapeutic regimens in clinics. Inside the tumor cell the core chromophore phenoxazinone ontercalates between the stacked nucleobases at the DNA guanine/cytosine sites whereas the pentapeptide elements bind to the minor groove. These binding interactions effectively inhibit replication and transcription processes in the tumor cells [93].

Actinomycin D, actinomycin V and actinomycin X0 $\beta$ were isolated from the Streptomyces sp. ZZ338 of marine origin and then tested for their activity in inhibiting the 
proliferation of human glioma U251 and SHG44 cells and rat glioma C6 cells. All tested compounds were shown to have high potency regarding inhibition of the cell proliferation in the three glioma cell lines when applied in nanomolar half maximal inhibitory concentration (IC50). Doxorubicin served as positive control have shown anti-glioma effects in IC50 range from 0.70 to $9.61 \mu \mathrm{M}$. In experimental conditions actinomycin D significantly downregulated the expression levels of hexokinase 2 (HK2), pyruvate kinase M2 (PKM2), glutaminase (GLS), and fatty acid synthase (FASN) [94]. These results suggest that glioma metabolic enzymes of glycolysis, glutaminolysis, and lipogenesis are the potential targets in the anticancer pathway initiated by actinomycins.

Table 11. The structure and antiproliferative activity of actinomycins D, V, and $\mathrm{X}_{\mathrm{op}}$ isolated from Streptomyces sp. ZZ338 against glioma cells [94].

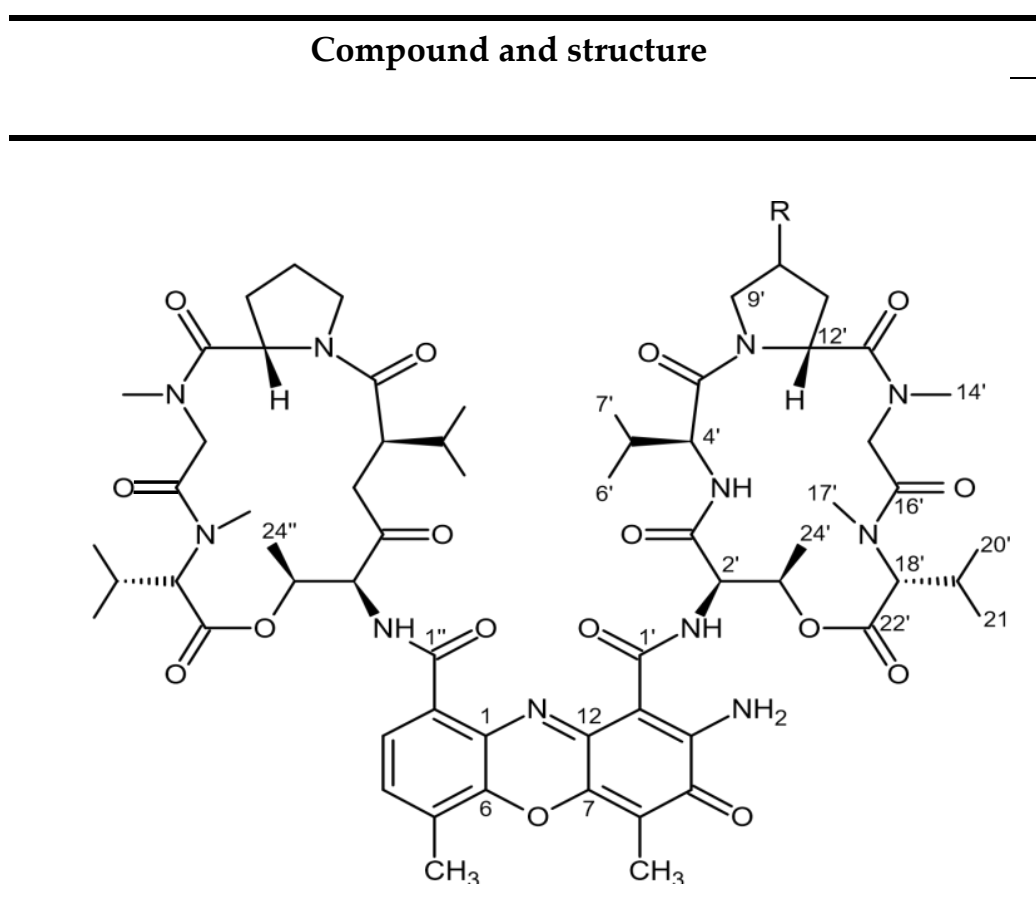

\begin{tabular}{llllc} 
Actinomycin $\mathrm{D}$ & $(\mathrm{R}=\mathrm{H})$ & $10.06 \pm 0.6 \mathrm{nM}$ & $3.31 \pm 0.25 \mathrm{nM}$ & $1.01 \pm 0.05 \mathrm{nM}$ \\
\hline Actinomycin $\mathrm{V}$ & $(\mathrm{R}=\mathrm{O})$ & $1.80 \pm 0.19 \mathrm{nM}$ & $1.37 \pm 0.07 \mathrm{nM}$ & $0.42 \pm 0.23 \mathrm{nM}$ \\
\hline Actinomycin $\mathrm{X}_{\mathrm{o}}$ & $(\mathrm{R}=\mathrm{OH})$ & $8.71 \pm 0.66 \mathrm{nM}$ & $3.26 \pm 0.32 \mathrm{nM}$ & $25.18 \pm 0.47 \mathrm{nM}$ \\
\hline Doxorubicin (positive control) & $9.61 \pm 1.25 \mu \mathrm{M}$ & $2.54 \pm 0.23 \mu \mathrm{M}$ & $0.70 \pm 0.01 \mu \mathrm{M}$ \\
\hline
\end{tabular}

Antimycins. Up to date more than forty natural antimycins A were discovered including deacyl antimycins exhibiting antifungal, anti-inflammatory, insecticidal, and anticancer activities [95]. Natural antimycins A contain unique nine-membered dilactone core conjugated with a 3-formyl aminosalicylic acid moiety.

Two recently discovered benzamide noncyclic dilactones neoantimycin A and neoantimycin B as well as already known antimycins A1ab, A2a and A9 were isolated from the culture broth of the actinomycete Streptomyces antibiotics H12-15 obtained from the sea sediment collected in mangrove area. Aforementioned compounds were investigated regarding their cytotoxic activity against human glioblastoma cells SF-268. Both neoantimycins have demonstrated moderate cytotoxic activity which was comparable to the one of anticancer drug cis-dichlorodiamine platinum. Antimycins A1ab, A2a and A9 contain 3-aminosalicylic acid and proved to be scientifically more potent by 20 and 26 times with IC50 values below $1.6 \mu \mathrm{g} / \mathrm{mL}$ (Table 12). According to the results of the cytotoxicity assay 
study [96], it may be concluded that dilactone ring may be directly involved in the interaction between antimycin $\mathrm{A}$ and its site of action structures.

Table 12. The structure and anti-glioblastoma activity of antimycins from the Streptomyces antibioticus H12-15 of marine origin against human glioblastoma cell line SF-268 [96].

\begin{tabular}{|c|c|c|}
\hline Compound & Structure & $\mathrm{IC}_{50}, \mu \mathrm{g} / \mathrm{mL}$ \\
\hline & $\overbrace{7}$ & \\
\hline Neoantimycin A & $\left(\mathrm{R}_{1}=\mathrm{CH}\left(\mathrm{CH}_{3}\right) \mathrm{CH}_{2} \mathrm{CH}_{3}\right.$ & 33.6 \\
\hline Neoantimycin B & $\left(\mathrm{R}_{1}=\mathrm{CH}\left(\mathrm{CH}_{3}\right)_{2}\right.$ & 41.6 \\
\hline
\end{tabular}

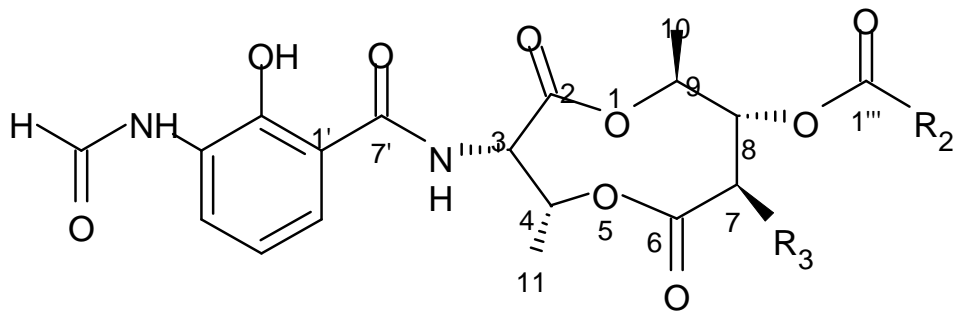

Antimycin $\mathrm{A}_{1 \mathrm{ab}}$ (a) $\quad\left(\mathrm{R}_{2}=\mathrm{CH}\left(\mathrm{CH}_{3}\right) \mathrm{CH}_{2} \mathrm{CH}_{3}\right), \quad \mathrm{R}_{3}=n \mathrm{Hex}$

(b) $\quad\left(\mathrm{R}_{2}=\mathrm{CHCH}_{2}\left(\mathrm{CH}_{3}\right)_{2}\right.$, $<1.6$

\begin{tabular}{lccc}
\hline Antimycin $\mathrm{A}_{2 \mathrm{a}}$ & $\left(\mathrm{R}_{2}=\mathrm{CH}\left(\mathrm{CH}_{3}\right)_{2}\right.$, & $\mathrm{R}_{3}=n \mathrm{Hex}$ & $<1.6$ \\
\hline Antimycin $\mathrm{A}_{9}$ & $\left(\mathrm{R}_{2}=\mathrm{CH}_{2} \mathrm{Ph}\right.$ & $\mathrm{R}_{3}=n \mathrm{Hex}$ & $<1.6$ \\
\hline $\begin{array}{l}\text { Cis-dichlorodiamine platinum } \\
\text { (positive control) }\end{array}$ & & 41.0 \\
\hline
\end{tabular}

Anthracyclines. Biologically active compound SZ-685C, an anthracycline analogue isolated from the secondary metabolite of mangrove endophytic fungus \#1403 collected in the South China Sea was teste for its antitumor activity in the glioma cell line LN-444. SZ-685C was shown to inhibit cell vitality of the glioma cells with IC50 around $7.8 \mu \mathrm{M}$ after 48 hours since drug administration (Table 13) [97]. It should be noted that anthracyclines make the whole class of the chemotherapeutic compounds isolated from Streptomyces and considered as ones of the most effective antitumor drug that were ever developed and used in the treatment of various human malignancies. Clinical investigation of anthracyclines is substantially restricted due to their serious adverse effects including cardiotoxicity and myelosuppression as well as high frequency of the multidrug resistance development. Therefore, the search of the novel anthracenedione derivatives with improved safety profile is one of the goals of modern oncopharmacology. Anthraquinone SZ-685C was selected from several dozen compounds that show significant inhibition of the malignant cells and mild cytotoxic influence regarding healthy cells.

It was shown in the human breast cancer cell lines, MCF-7 and MDA-MB-435 that SZ-685C compound selectively kills cancer cells via activation of the both caspase-8- and 
caspase-9-based apoptotic pathways by suppression of the whole or at least in part of the AKT phosphorylation. The AKT pathway plays a key role in sustaining survival against the apoptotic death programming in cancer cells. It has been shown in numerous studies that AKT hyperactivation is a frequent event typical of many human cancer types and activation of the phosphatidylinositol 3-kinase (PI3K)/AKT pathways has been reported to contribute to chemotherapeutic resistance in human cancers. That suggest that this pathway is an essential target for anticancer therapies, and development of effective as ta the same time safe therapeutic inhibitors of PI3K or Akt still remains a major challenge of antitumor pharmacology. SZ-685C inactivates AKT in the breath cancer cells that in turn results in reduced FOXO1 and FOXO3a phosphorylation and consequently leads to increased expression of its downstream target genes, such as Bim. Up-regulation of Bim, which can bind to Bcl-2 and Bcl-XL via its BH3 domain, thereby sequestering Bcl-2 from the pro-apoptotic Bax and Bad, and initiating the apoptotic cascade, has been seen in breast cancer cells treated by SZ-685C [97]. Despite SZ-685C is already considered as promising anti-AKT and antitumor drug candidate, the mechanisms of its cytotoxic effects regarding glioma cells are not cleared out yet.

Table 13. The structure and anti-glioma activity of the anthracycline analogue SZ-685C from mangrove endophytic fungus No. 1403 against glioma cell line LN-444 [97].

Compound

Bagremycins. A crude extract prepared from a culture of a mangrove-derived actinomycete, Streptomyces sp. Q22, isolated from a sample of mangrove soil was found to be active against human glioma cells. A large culture of this actinomycete in liquid medium resulted in isolation of eight compounds, bagremycins $\mathrm{A}, \mathrm{B}, \mathrm{C}, \mathrm{D}$, and E, bagrelactone $\mathrm{A}$, and two styrene derivatives (4-hydroxystyrene and 4-hydroxystyrene 4-O- $\alpha$-Dgalactopyranoside). Bagremycins $\mathrm{C}-\mathrm{E}$ and bagrelactone $\mathrm{A}$ were found to be new compounds. The activity of all isolated compounds against four different glioma cell lines, U87MG, U251, SHG44, and C6, was assayed. The results indicated bagremycin C was active against four different glioma cell lines, with IC50 values in a range from 2.2 to $6.4 \mu \mathrm{M}$ $(72 \mathrm{~h})$, compared to the activity of the doxorubicin $(0.4$ to $3.3 \mu \mathrm{M})$ (Table 14$)$. Bagremycin $\mathrm{B}$ also showed activity against the glioma cells with IC50 values of 7.3 to $13.3 \mu \mathrm{M}$ [98].

Table 14. The structure and inhibitory activity of bagremycins B and C from a mangrove-derived actinomycete, Streptomyces sp. Q22 against glioma cells lines [98].

\begin{tabular}{llllll}
\hline Compound & Structure & \multicolumn{4}{c}{$\mathrm{IC}_{50}(\mu \mathrm{M})$} \\
\cline { 3 - 6 } & & U87MG & U251 & SHG44 & C6 \\
\hline
\end{tabular}

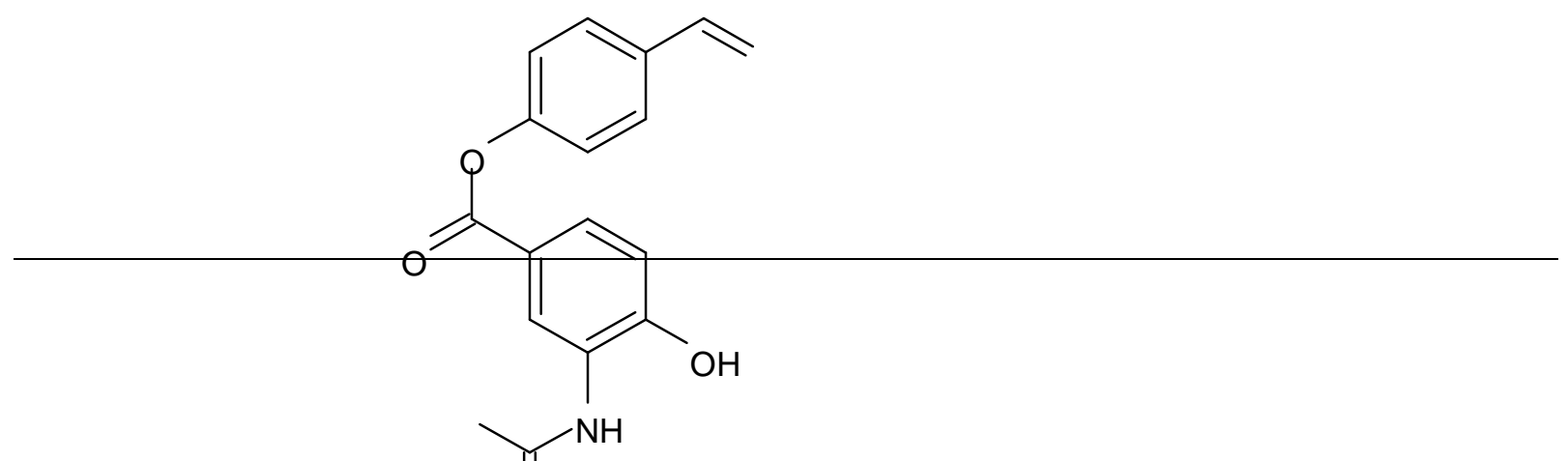


Bagremycin B

$10.2 \pm 0.5 \quad 9.7 \pm 1.9 \quad 7.3 \pm 0.8$

$13.3 \pm 2.4$

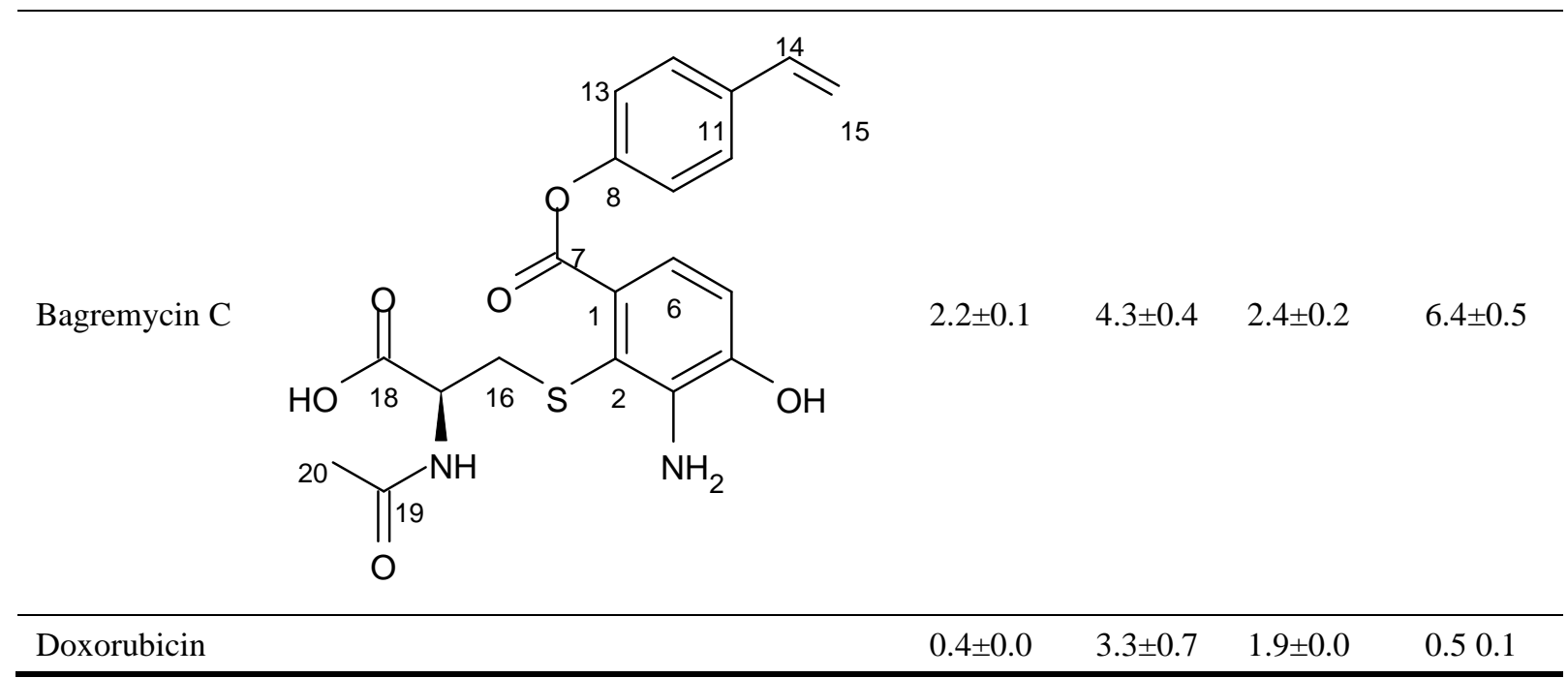

Bagremycins are phenol esters of p-hydroxystyrene and p-hydroxybenzoic acid. Bagremycin C molecular formula was found to be as C20H20N2O6S with three main subunits making its structure such as p-hydroxystyrene, 3-amino-4hydroxybenzoic acid, and $\mathrm{N}$-acetylcysteine. Such bagremycin presents a unique analogue with an N-acetyl-(S)-cysteine moiety, that probably makes bagremycin $C$ the one of the most potent antigliomic agent among all the compounds studied up to date. The configuration at C-17 in bagremycin $C$ was suggested to be $S$ by a comparison of its negative optical rotation value with those of known compounds $\mathrm{N}$-acetyl-(S)-cysteine and $\mathrm{N}$-acetyl(R)-cysteine, where a negative value was suggestive of an $S$-configuration, while a positive value indicated an $R$ configuration.

Bagremycin $\mathrm{C}$ was found to induce apoptosis in human glioma U87MG cells in a dose- and time-dependent manner, and provoke the U87MG cell cycle arrest at the G0/G1 phase. The total number of apoptotic cells induced by bagremycin $\mathrm{C}$ was increased by $20.59 \%(48 \mathrm{~h})$ and $58.32 \%(72 \mathrm{~h})$ at $2.2 \mu \mathrm{M}$ and $61.16 \%(48 \mathrm{~h})$ and $93.00 \%(72 \mathrm{~h})$ at $4.4 \mu \mathrm{M}$, respectively, compared to the control ( $5.80 \%$ for $48 \mathrm{~h}$ and $5.24 \%$ for $72 \mathrm{~h}$ ). The doxorubicin $(10.0 \mu \mathrm{M})$ induced apoptosis in the U87MG cells by $10.42 \%$ (48 h) with $26.87 \%$ (72 h) increase of total apoptotic cells. Moreover, cell cycle assay showed that the cell population at the G0/G1 phase was enhanced by $16.18 \%$ and $20.56 \%$ after $12 \mathrm{~h}$ of exposure respectively to 4.4 and $8.8 \mu \mathrm{M}$ bagremycin $\mathrm{C}$ concentration [98]. These changes occurring in the cell cycle indicated that bagremycin $C$ might block the glioma cell cycle at the G0/G1 phase.

Capoamycins. Antibiotic agent capomycin was originally isolated from the culture broth of soil Streptomyces capoamus strain No. 23-41. Its characteristic structure consists of a modified benz[a]anthraquinone chromophore, a deoxysugar unit and a long chain polyene acid (Figure 7). Capoamycin has been reported to inhibit the growth of grampositive bacteria, yeasts and fungi as well as it induces differentiation of mouse myeloid leukemia cells, and prolongs survival periods of mice with xenografted Ehrich ascites carcinoma [99]. Capoamycin type antibiotic compound MK844-mF10 (molecular formula C38H38O13) was initially isolated from the soil strain of Streptomyces sp. MK844-mF10 and later identified in the cultural media of the Streptomyces fradiae PTZ00025 strain found in the marine precipitates along with two novel compounds соединениями, 
named as fradimycin A (molecular formula C39H42O15) and fradimycin B (molecular formula C38H38O14).<smiles>CCCCC/C=C/C=C/C(=O)O[C@H]1[C@@H](O)C[C@@H](c2ccc3c(c2O)C(=O)C2=C(C3=O)[C@]3(O)C(=O)C=C(C)C[C@]3(O)C=C2)O[C@@H]1C</smiles>

Figure 7. The structure of capoamycin

All three marine antibiotics have shown strong dose-dependent activity toward inhibition of the glioma C6 cell growth in the concentration range between 0.125 and $40 \mu \mathrm{M}$ with the average IC50 values from 0.47 to $1,31 \mu \mathrm{M}$ (Table 15). Fradimycin B was found to be the most active compound in concentration between 0.625 and $1.25 \mu \mathrm{M}$ and it induced apoptosis and necrosis in C6 cells as well as it blocked cell cycle at the phase G0/G1 in the test human colon HCT-15 cell line [100]. Polyenoic acids assigned as fradic acid A and fradic acid B were isolated with fradimycins from Streptomyces fradiae PTZ00025 and later found to be inactive.

Table 15. Structure and cytotoxicity activity of the capoamycin-type antibiotics from marine Streptomyces fradiae PTZ0025 against glioma C6 cells [100].

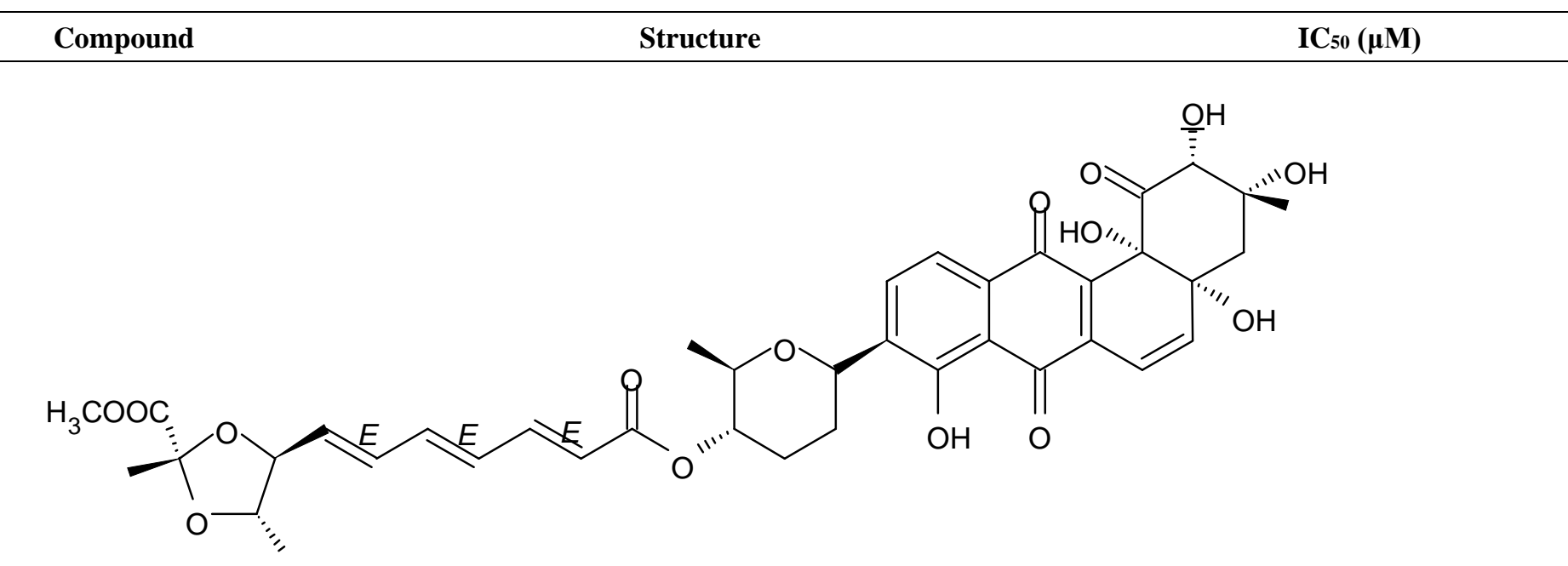

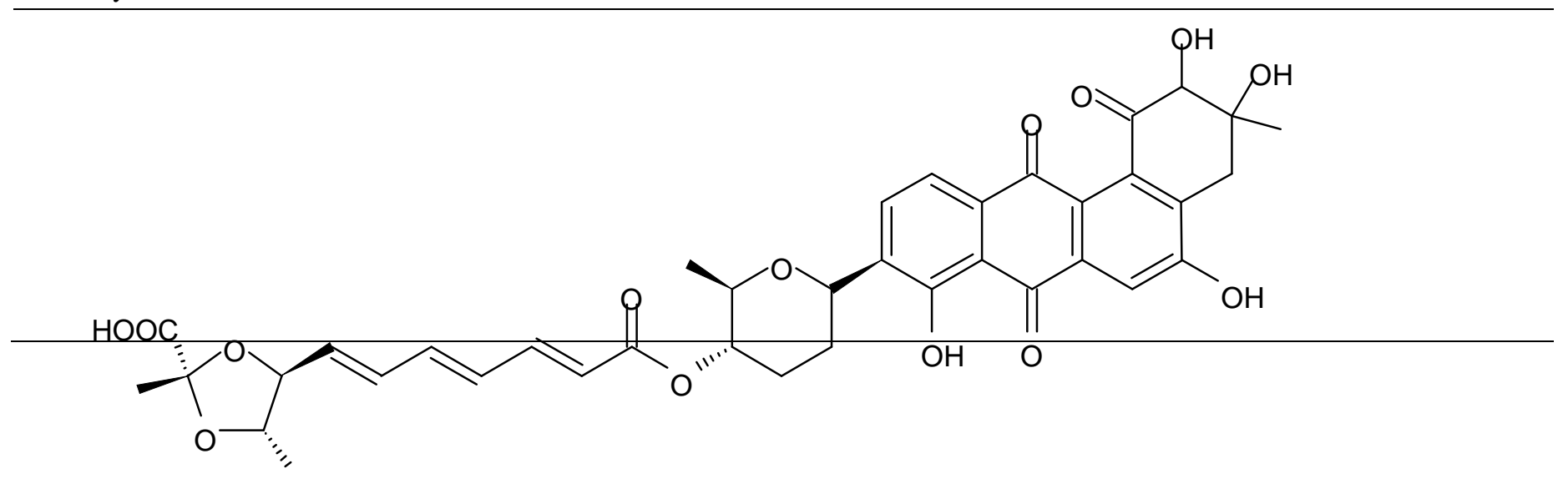


<smiles>Cc1cc2c(c3c1CC(C)(O)C(O)C3=O)C(=O)c1ccc([C@H]3CC[C@H](OC(=O)/C=C/C=C/C=C/[C@H]4O[C@@](C)(C(=O)O)O[C@H]4C)[C@H](C)O3)c(O)c1C2=O</smiles>

Polyene-polyol macrolides. Polyene-polyol macrolides are mainly microbial metabolites with the main structural elements presented as a continuously conjugated chain of four to seven unsubstituted double bonds connected to a polyol fragment with alternating hydroxy groups.

The most widely used antibiotics belonging to this group are nystatin, a hexaenepolyol macrolide produced by Streptomyces noursei, and amphotericin B, a heptaene-polyol macrolide produced by S. nodosus, both showing antifungal activity. Despite the major part of the polyen-polyol macrolides are synthesized by the terrestrial species of the Streptomyces, these macrolides were also isolated from marine actinomycetes. Some of these polyene-polyol macrolides were found to have significant antifungal, antibacterial, antiviral, and antitumor activities [101]. During the search for the new antioglioma antibiotic in the marine resources, among ten bacterial strains isolated from a sample of mangrove soils, extract made of Streptomyces sp. ZQ4BG was found to be the most active one against the proliferation of glioma cells U87MG and U251. Several polyene-polyol macrolides including well-known flavofungins I and II previously isolated from the actynomicet Streptomyces flavofungini, was found and identified as well as flavofungins III - IX previously known as spectinabilin, a nitro-containing metabolite first isolated from Streptomyces spectabilis. Chemical structures of the new compounds isolated from ZQ4BG are given in the Table 16.

Flavofungins I and II were 32-membered polyene-polyol macrolides with a conjugated chain of five unsubstituted double bonds connected to a polyol fragment with eight hydroxy groups alternated. New compounds named as flavofungins III-IX are new members of flavofungins with only four unsubstituted conjugated double bonds and more oxygenated moieties. Flavofungin III is an analogue of flavofungin I with two hydroxy groups substituted at C-10 and C-11. Flavofungins IV-IX are rare 32-membered polyenepolyol macrolides with a tetrahydrofuran ring between C-10 and C-13 for flavofungins IV-VIII or a unique oxepane group (ring) between C-10 and C-15 for flavofungin IX.

All ten isolated compounds were evaluated for their activity in inhibiting proliferation of glioma cells U251, U87MG, SHG44, and C6, and doxorubicin was used as a positive control. Flavofungin II and spectinabilin showed moderate activity against the proliferation of four tested glioma cell lines. Flavofungin I showed weak activity (Table 16). Flavofungins III-IX were inactive. The cytotoxicity (CC50) of the flavofungin II and spectinabilin towards normal human astrocyte and human foreskin fibroblast 1 cells were also assayed. Both compounds showed a higher selectivity index (CC50/IC50) for normal 
astrocytes with 4.6-16.5 for flavofungin II and 3.4-13.3 for spectinabilin, when compared to the selectivity index for fibroblasts with 1.8-6.4 for flavofungin II and 1.3-5.1 for spectinabilin [102].

Table 16. The structure and inhibitory activity of polyene-polyol macrolides from Streptomyces sp. ZQ4BG against glioma cells lines U251, U87MG, SHG44, and C6 [102].

Compound

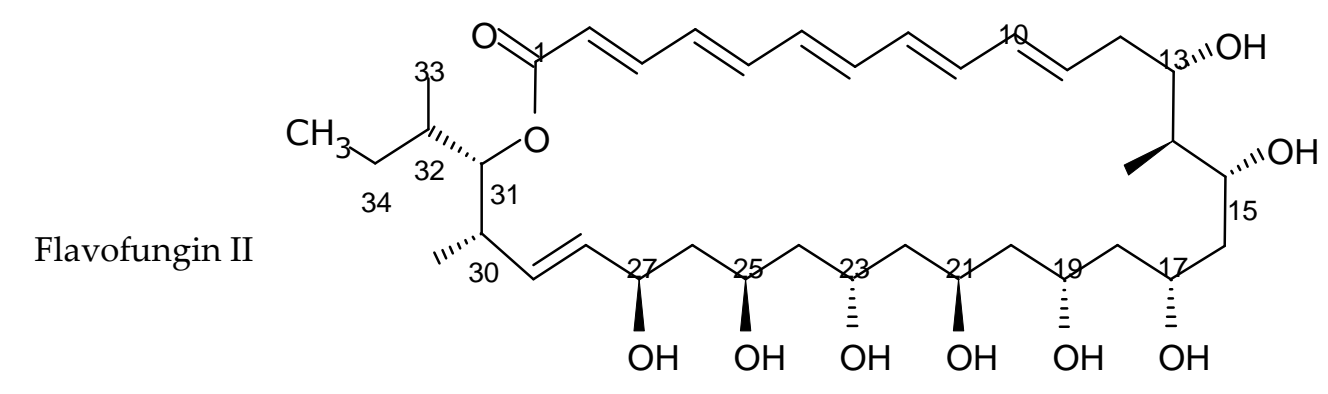

Spectinabilin

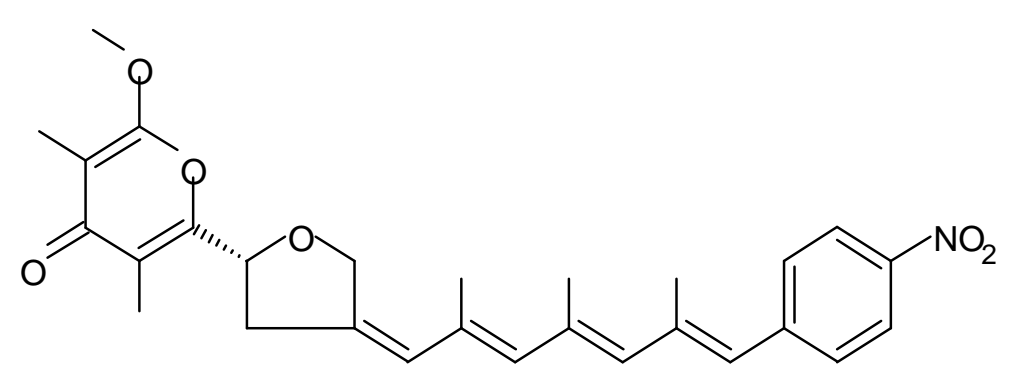

$10.86-42.75$

\section{Saponins}

Saponins are the natural glycosides with triterpene or steroid aglycon. That is why the following terminology is designated to saponins in scientific literature: triterpene glycosides (or triterpene saponins) and steroid glycosides (or steroid saponins). Even recently saponins were considered typical plant metabolites but nowadays these substances are also thought as metabolites of the marine origin found in two classes of the phylum Echinodermata such as Asteroidea and Holothuroidea. Steroid saponins are generally contained in starfishes, while triterpenoid saponins are mainly evidenced in sea cucumbers. Steroid saponins are divided into three groups: asterosaponins, polyhydroxysteroidal glycosides and basically rare type of these compounds cyclic glycosides. Asterosaponins have typical structural patterns including an aglycone with $\Delta 9(11), 3 \beta, 6 \beta$ - 
dihydroxysterodal nucleus and a sulfate group at C-3. The oligosaccharide chain generally is comprised of five or six sugar units glycosidically linked at C-6 and is made up of the structural diversity of asterosaponins. The sugar moieties of saponins are mostly presented with D- or L-forms of arabinose (Ara), fucose (Fuc), quinovose (Qui), xylose (Xyl), galactose (Gal), and glucose (Glc) [103].

Steroid saponins. The first research study focused on antiglioma activity of the marine saponins [104], showed the such compound as asterosaponin 1 isolated from the starfish Culcita novaeguineae and formulated as sodium (20S)-6 $\alpha$-O-\{ $\beta$-D-fucopyranosyl$(1 \rightarrow 2)$ - $\alpha$-L-arabinopyranosyl-( $1 \rightarrow 4)$-[ $\beta$-D-quinovopyranosyl-( $1 \rightarrow 2)]-\beta$-D-xylopyranosyl$(1 \rightarrow 3)-\beta$-D-quinovopyranosyl\}-20-hydorxy-23-oxo-5 $\alpha$-cholest-9(11)-en-3 $\beta$-yl-sulfate) (molecular formula as C55H89O27SNa, molecular weight 1236) when dosed in a range from 2.5 to $20 \mu \mathrm{g} / \mathrm{mL}$ significantly suppressed U87MG cell proliferation in a time- and dose-dependent manner (Table 17).

Table 17. The structure and cytotoxicity of asterosaponin 1 from the starfish Culcita novaeguineae on human glioblastoma U87MG cells [104].

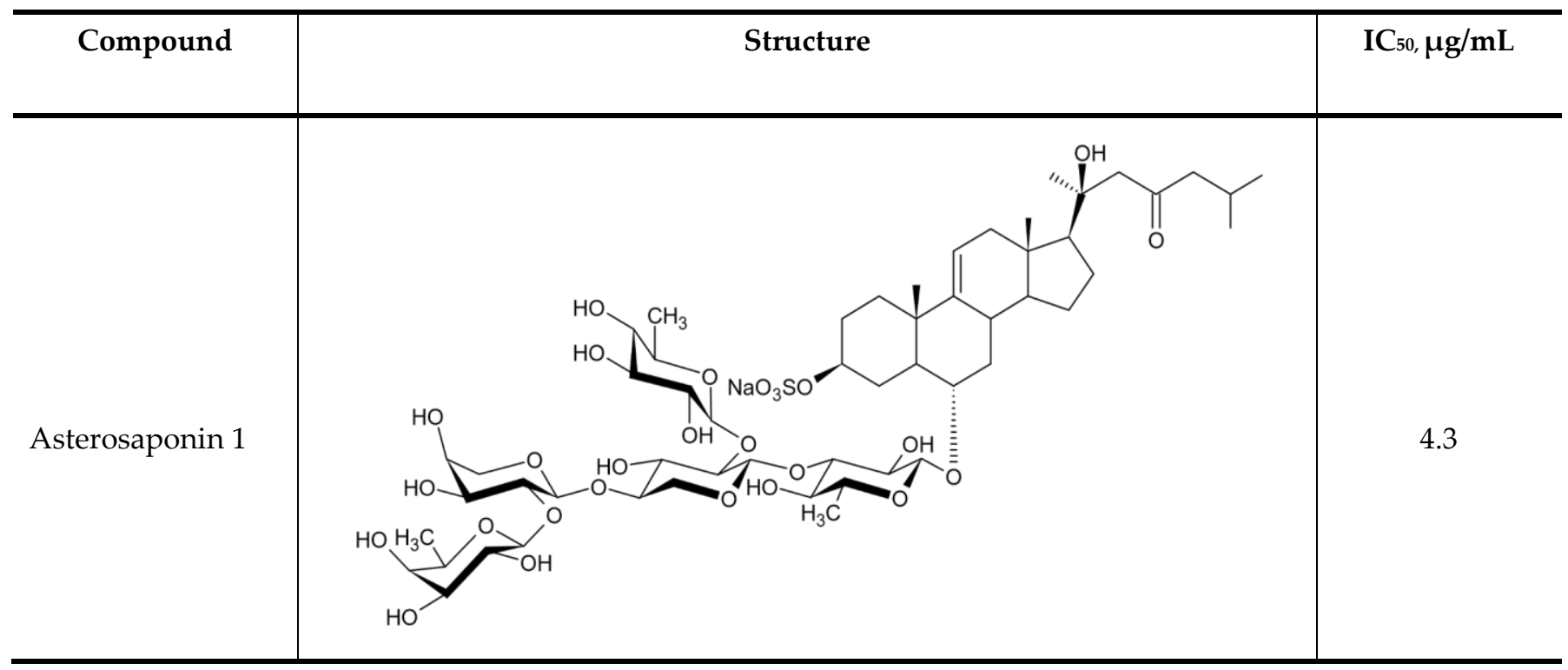

Antitumor effects of saponins may be generally caused by various pathways including apoptosis induction, autophagy, cytoskeleton disintegration, inhibition of angiogenesis and metastasis.

Treatment with 3.4 and $4.3 \mu \mathrm{g} / \mathrm{mL}$ of asterosaponin 1 led to induced S phase arrest, whereas application of $10.0 \mu \mathrm{g} / \mathrm{ml}$ of asterosaponin 1 resulted in the G0/G1 phase arrest. Percentage of cells being in the G2/M phase was decreased, while the number of cells in G0/G1 cycle phase increased after $24 \mathrm{~h}$ of the treatment start. In this study the glioma cells demonstrated typical morphological apoptosis signs such as nuclear condensation with apoptotic bodies and DNA fragmentation. Furthermore, asterosaponin 1 decreased the expression of Bcl-2 protein and increased the expression of the Bax protein [104].

It was shown later that at least four more asterosaponins isolated from the starfish C. novaeguinea exrted significant inhibiting activity against various human and mouse glioblastoma cell lines in vitro, but did not affect the primary cultured human astrocytes (Table 18), suggesting good safety profile of asterosaponins in vitro. The results suggest that $20 \alpha$-hydroxy is an effective functional group determining anti-glioblastoma activity of asterosaponins. Three asterosaponins exhibited tubulin polymerization activity, and one of them initially affected microtubule function and then blocked U87MG cell cycle resulting in mitochondrial damage, cristae disorganization, membrane potential collapse, cytochrome $\mathrm{C}$ release, and finally activated caspase 3 and induced apoptosis [103]. 
Apart from asterosaponins, there are four new polyhydroxysteroidal glycosides called culcinosides A, B, C and D presenting along with three known glycosides identified as echinasteroside $\mathrm{C}$, linckoside F, and linckoside L3. These compounds were isolated from the ethanol extract of starfish $C$. Novaeguineae and their cytotoxicity was evaluated on the human glioblastoma cell lines U87, U251, and SHG44. Generally polyhydroxysteroidal glycosides consist of an oxygenated steroidal aglycone with more than three hydroxy groups, and one or two (rarely three) monosaccharide residues attached to the steroidal nucleus or side chain. The strongest and highest cytoxic Culcinoside A had shown the most potent and strong cytotoxic activity. Culcinosides B, C, and D demonstrateded moderate activity (Table 19). Average IC50 for doxorubicin tested on the same cell models were approximately 28.3, 47 and 53.6 times than those of culcinoside A [105]. Eleven novel polyhydroxysteroid glycosides ranging from anthenoside $\mathrm{A}$ to anthenoside $\mathrm{K}$ were isolated from the starfish Anthenea chinensis. Seven of them were found to exert selective inhibiting activity against human tumor K-562 and BEL-7402 cells. Three of these glycosides were active in the human glioma U87MG cell lines. Anthenoside A was shown to have substantially high cytotoxicity against U87MG cells (Table 20) [103,106,107]. In addition, it exhibited tubulin polymerization promoting activity [106]. The structure-activity relationship analysis indicated that the monosaccharide units and their attachment sites on aglycone might be considered important for promoting tubulin polymerization and inhibiting glioma cell proliferation [107].

Table 18. The structure of asterosaponins from starfish Culcita novaeguineae and their inhibiting activity against human and mouse glioblastoma cell lines and human astrocytes [103].

\begin{tabular}{c|c|c|c|c|r|r}
\hline \multirow{2}{*}{ Sample and structure } & \multicolumn{7}{c}{ IC $50(\mu \mathrm{M})$} \\
\cline { 2 - 8 } & U87MG & U251MG & BT325 & SHG44 & C-6 & Astrocytes \\
\hline
\end{tabular}

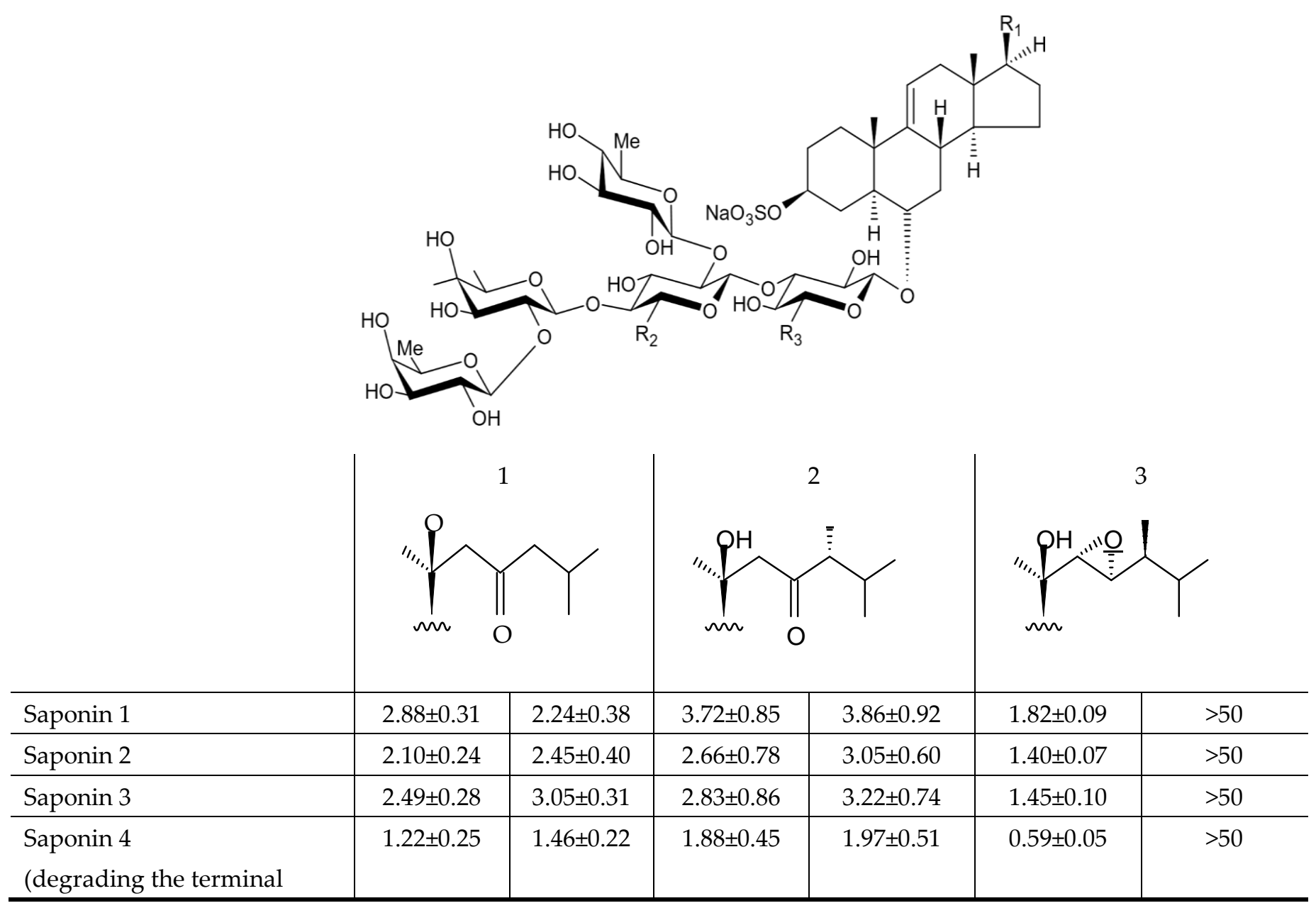




\section{$D$-fucose based on saponin 1)}

Table 19. The structure of polyhydroxysteroidal glycosides from starfish Culcita novaeguinea and their cytotoxic activity against glioblastoma cell lines in vitro [105].

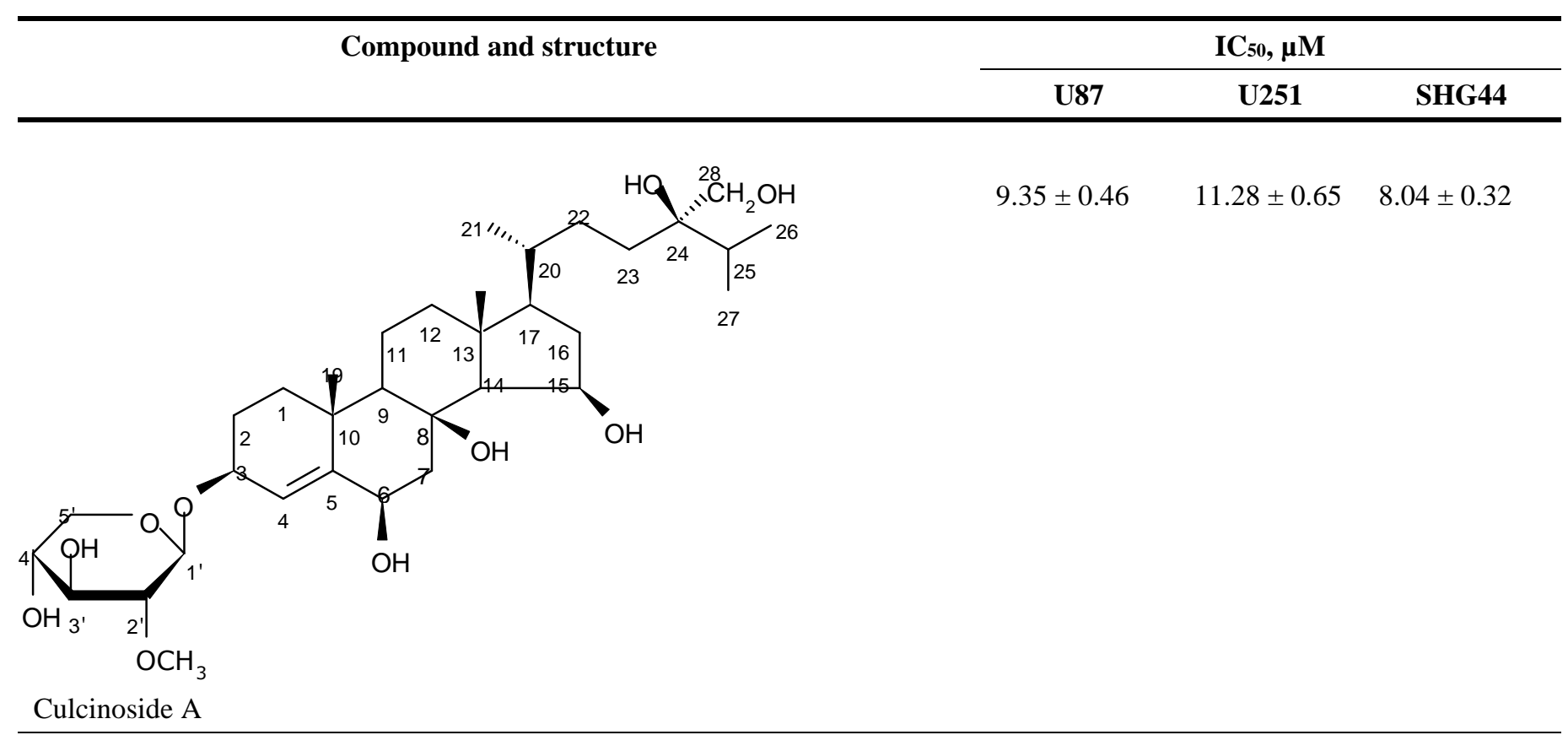

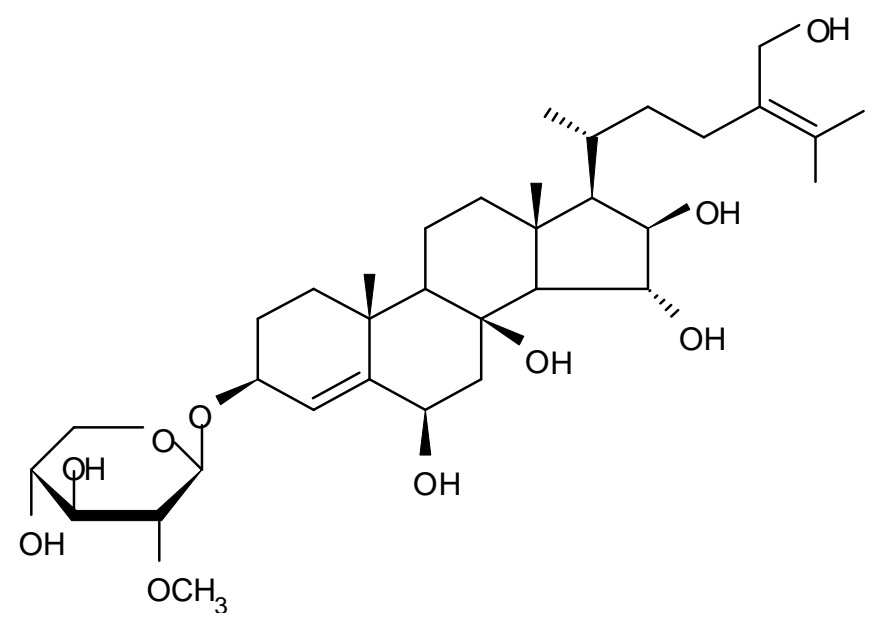

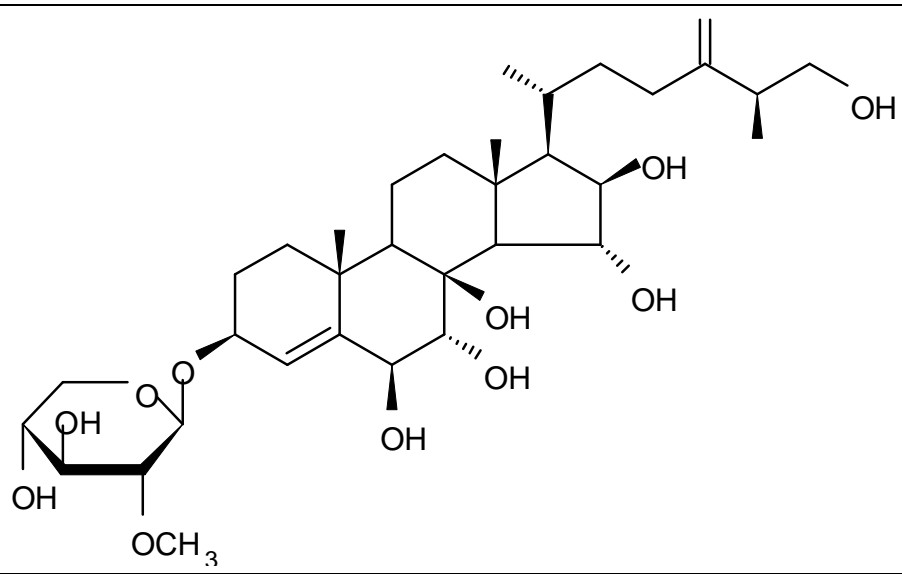




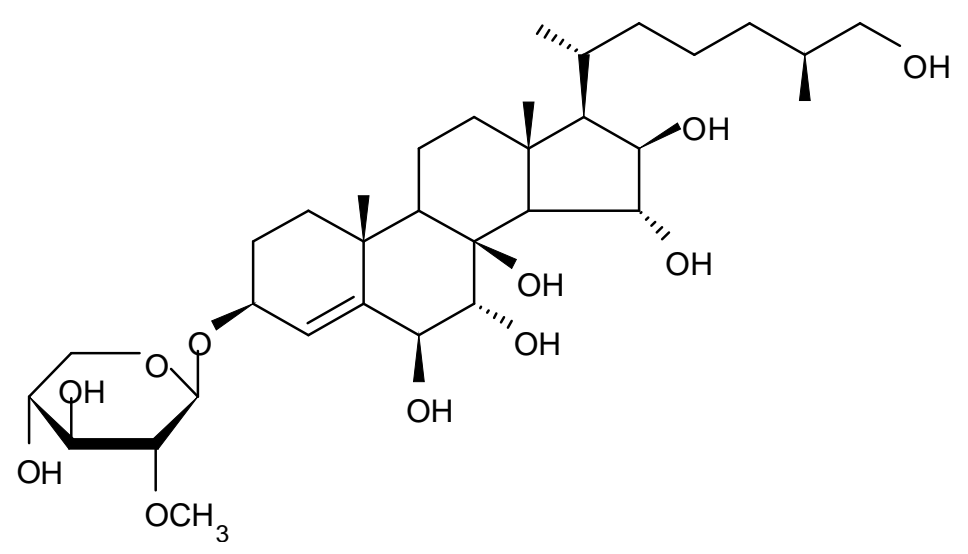

$43.25 \pm 1.73 \quad 28.93 \pm 1.83 \quad 26.22 \pm 1.64$

Culcinoside D

\begin{tabular}{lccc}
\hline Doxorubicin & $0.33 \pm 0.02$ & $0.24 \pm 0.01$ & $0.15 \pm 0.01$ \\
\hline
\end{tabular}

Table 20. The structure of anthenoside A from the starfish Anthenea chinensis and their cytotoxic activity against glioblastoma U87MG cells [106].

Compound

New asterosaponin and polyhydroxysteroid saponin found in star fish Pentaceraster chinensis (Figure 8) exerted strong cytotoxicity in the U87MG cell lines resulting in apoptosis modulated up-regulation of Bax protein and down-regulation of Bcl-2 protein [108]. 

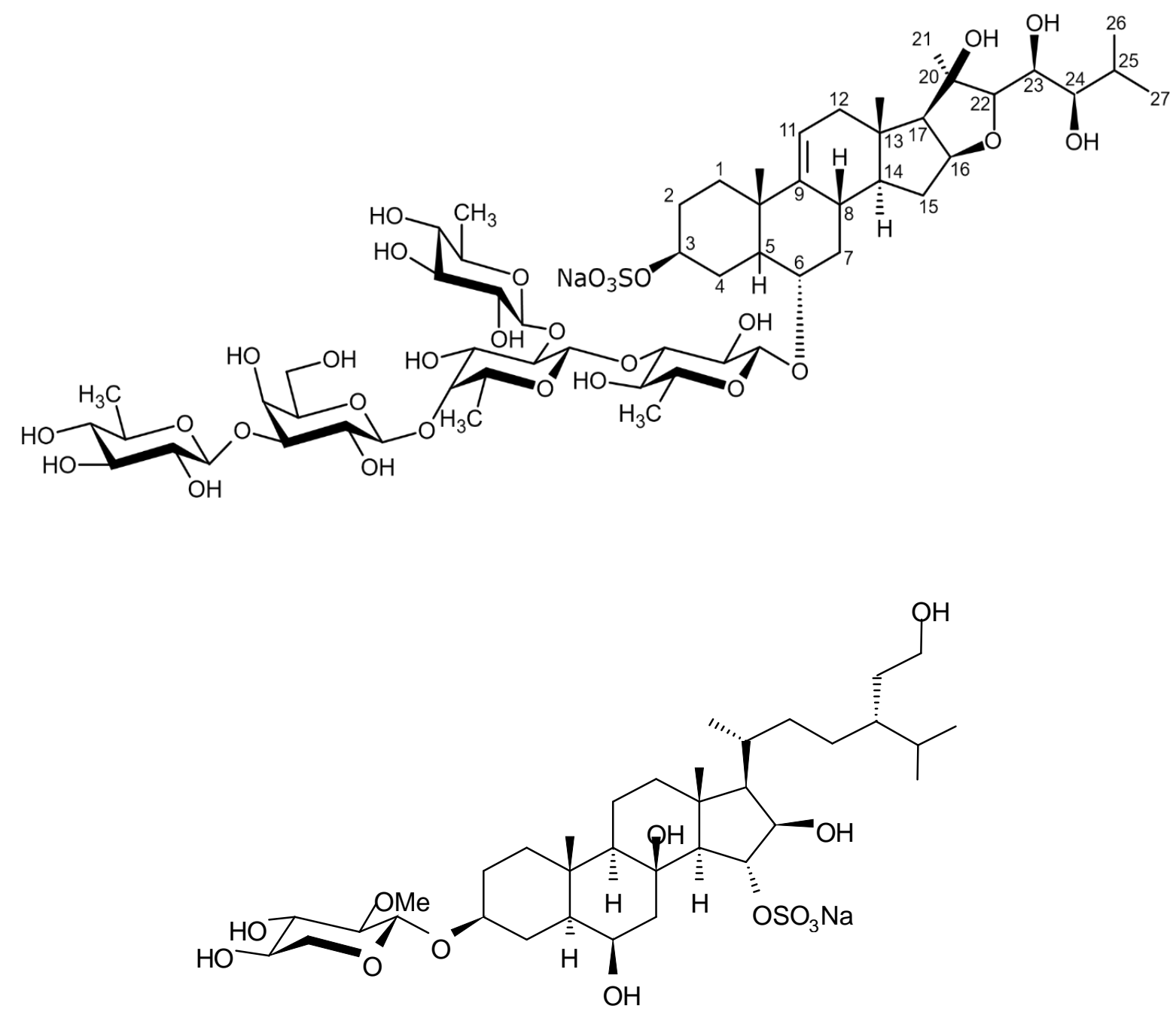

Figure 8. Asterosaponin and polyhydroxysteroidal glycoside from Pentaceraster chinensis exert antitumor activity against U87MG glioma cell line.

Triterpenoid saponins. Holothurian triterpene glycosides composed of oligosaccharide chain and holostane3-ol based aglycone often contain double bond between C-9 and C-11 or between C-7 and C-8 in the aglycone. Carbohydrate chain encloses up to 6 sugars units including xylose, glucose, 3-O-methylglucose, and quinovose that can be branched only once. The inter-specific differences in the triterpene glycoside structure are about the presence or absence of sulfate groups attached to the carbohydrate chain. More than 350 triterpenoid saponins from the sea cucumbers described so far, and a plenty of the possess cytotoxicity against various cancer cell lines [32].

The new holostane-type triterpene glycosides, namely pentactasides I, II and III, and two previously known glycosides philinopsides A and B were isolated from the sea cucumber Pentacta quadrangularis. Pentactasides I and II have the same trisaccharide moiety whereas pentactaside III contains sulfated diglycoside. Despite structural differences all the isolated glycosides inhibited U87MG glioma cell line with IC50 ranging between 1.90 and $3.95 \mu \mathrm{M}$ (Table 21) [109].

Table 21. The structure of triterpene glycosides from Pentacta quadrangularis and their cytotoxicity against glioblastoma U87MG cell lines in vitro [109].

Compound Structure


1 - Pentactaside III

$3.95 \pm 0.53$

2 - Pentactaside I

3 - Pentactaside II

4 - Philinopside A

5 - Philinopside B

(positive control)

The new triterpene glycoside fuscocineroside A shown on figure 9 was isolated from the sea cucumber Holothuria fuscocinerea. It inhibited U251 cell proliferation in a doseand time-dependent manner, induced apoptosis, and reduced surivin expression in the glioma cells, but at the same time, showed slight cytotoxicity regarding normal human astrocytes [110].

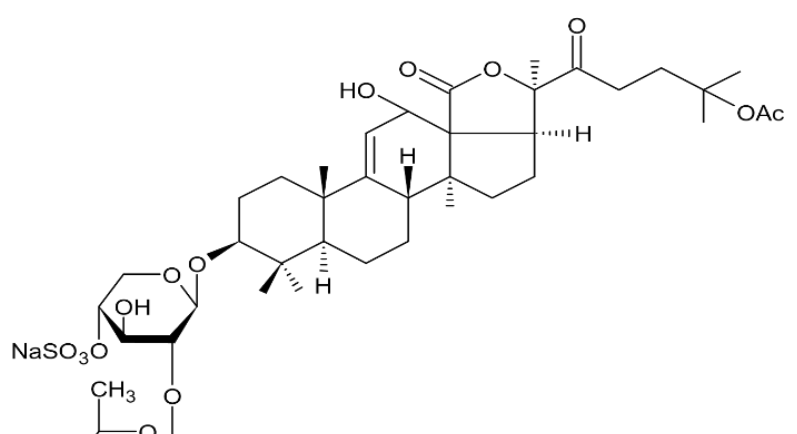


Figure 9. Fuscocineroside A from the sea cucumber Holothuria fuscocinerea exerting antiproliferative activity regarding glioma U251 cells [110].

Three new holostan-type triterpene glycosides given on figure 10 were isolated from the sea cucumber Bohadschia marmorata and induced significant inhibition of cell proliferation in five tumor lines including human glioma U87MG cells [103].

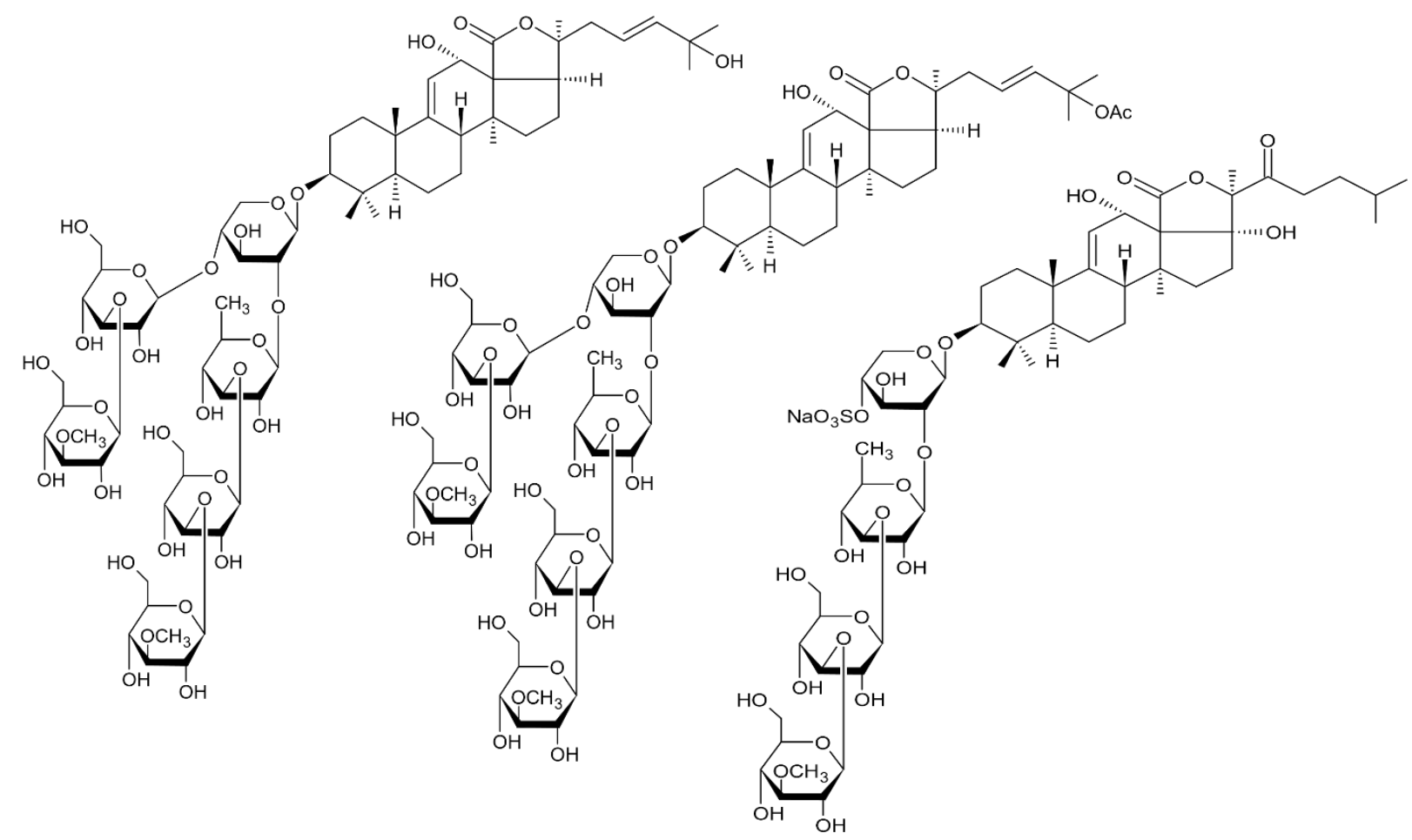

Figure 10. The triterpene glycosides from the sea cucumber Bohadschia marmorata exerting antiproliferating activity against glioma U87MG cells [103].

Another holostan-type triterpene glycoside, pervicoside D (Figure 11) was isolated from the sea cucumber Holoturia (Microthele) axiloga [Yuan et al., 2009] and was confirmed to exhibit significant inhibiting activity against human glioma U87MG cells [103].

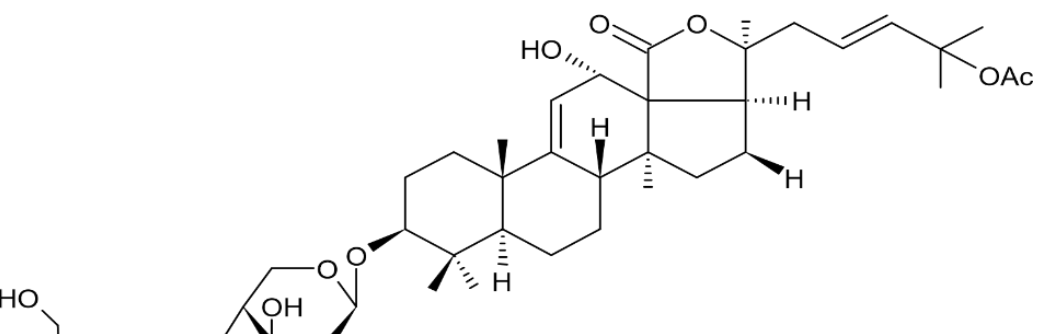


Figure 11. Pervicoside D from the sea cucumber Holoturia axiloga [103].

Ethyl acetate extract fraction of the sea cucumber Holothuria scabra body wall was applied in a dose range from 1 to $100 \mathrm{mkg} / \mathrm{ml}$ resulting in the substantial reduction of the cell vitality of the human glioblastoma lines A172 and U87MG with IC50 4.23 and 4.46 $\mu \mathrm{g} / \mathrm{mL}$, respectively, in 24 hours. Furthermore, that extract was shown to exert cytotoxicity greater than antitumor drug temozolomide on both cell lines at the same concentrations. This extract did not exhibit toxicity regarding primary human fetal astrocytes in the concentration range between 0,1 and $5 \mu \mathrm{g} / \mathrm{mL}$. In addition, extract induced early and late apoptosis stages, loss of the mitochondrial membrane potential, nuclear condensation and fragmentation.

Treatment of the glioblastoma cells with aforementioned extract for 24 hours results in increased expression of proapoptotic Bax and caspase-3 whereas Bcl-2 expression was suppressed in a concentration-dependent manner. The following major morphological changes typical of apoptosis were observed: shortening of the cell processes, detachment and loss of confluence resulting in the cell round-ups, cytoplasmic condensation, and formation of apoptotic bodies. Results of the acetyl acetate holothurian extract separation using HPLC with the following LC-MS/MS data analysis have shown that the extract contain a group of compounds, which mass-spectrum was corresponding to the one of triterpene glycosides identified earlier in the other sea cucumber species. Scabrasides A and B and holothurin A3 found in the sea cucumber Holothuria scabra were identified in greater amounts whereas in the less quantities were found substances corresponding triterpene glycosides from H. forskali, H. lesson, Athyonidium chilensis and Apostichopus japonicus [112].

Already known sulphated saponins holothurin A, holothurin B, and 24-dehydroechinoside $B$ as well as new triterpenoid saponin (3-O-[ $\beta$-D-quinovopyranosyl- $(1 \rightarrow 2)-4$-sodium sulfato- $\beta$-D-xylopyranosyl]-25-acetoxy-22-oxo-9(11)-holostene-3 $\beta, 12 \alpha, 17 \alpha$-triol), isolated from the sea cucumber Holothuria moebii suppressed the proliferation of human glioma U87-MG, U251, and SHG-44 cells and rat glioma C6 cells with IC50 values ranging from 0.99 to $8.64 \mu \mathrm{M}$ (Table 22). An artificial compound desulfated holothurin B had shown moderate or weak activity with IC50 values from $14.43 \pm 1.33$ to $53.01 \pm 1.64 \mu \mathrm{M}$ [113]. That is very likely that the sulfate group at C-4 of xylose is important for the activity of this type of triterpenoid saponins. Temozolomide exerted light activity regarding C6 and SHG-44 cells and did not show any activity on U87-MG and U251cells at $100 \mu \mathrm{M}$ probably due to the cell resistance to temozolomide. 
Recently discovered saponin was applied in concentrations 2 and $4 \mu \mathrm{M}$ inducing apoptosis and necrosis in U87-MG cells in 24 hours. Increase of the apoptotic cell number was $60.25 \%(2 \mu \mathrm{M})$ and $55.02 \%(4 \mu \mathrm{M})$, increase of necrotic cell amount was $0.23 \%(2 \mu \mathrm{M})$ and $10.47 \%(4 \mu \mathrm{M})$, respectively, whereas total number of both apoptotic and necrotic cells was $60.48 \%(2 \mu \mathrm{M})$ and $65.49 \%(4 \mu \mathrm{M})$ higher in comparison to control. Investigation of the effects exerted by the new sulfated saponin on proapoptotic genes BCL-2/BCL-XI-associated death promoter (BAD) and BCL-2-associated $X$ protein (BAX) and on anti-apoptotic genes B-cell lymphoma 2 (BCL-2) and B-cell lymphoma-extra large (BCL-XL) showed this saponin does not regulate expression level of both pro- and anti-apoptotic genes. The results of the study devoted to the influence of this sulfated saponin on the expression levels of glioma metabolic enzymes of glycolysis and glutaminolysis demonstrated that this compound $(4 \mu \mathrm{M})$ significantly lowers the expression levels of hexokinase 2 (HK2), 6-phosphofructo-2-kinase/2,6-bisphosphatase 3 (PFKFB3), pyruvate kinase (PKM2) and glutaminase (GLS) in U87-MG cells after $24 \mathrm{~h}$ treatment [113]. At the same time, it did not affect expression of the enzyme in the normal human astrocytes. It did not influence the expression levels of aconitase 2 (ACO 2), ATP synthase beta (ATPB), pyruvate dehydrogenase beta (DPHB), and cytochrome $\mathrm{C}(\mathrm{CytoC})$ as well [113], which are important regulators in the processes of the tricarboxylic acid cycle and oxidative phosphorylation involved into the glucose metabolism in normal cells. This makes a suggestion that holothurian sulfated saponins have unique antitumor mechanism selectively targeting glioma metabolic regulators of glycolysis and glutaminolysis.

Having discussion about substantial antiglioma activity of saponins in vitro, it should be pointed out that systemic administration of the pharmaceutical containing saponins us barely possible due to such their side effect as hemolysis [114]. However due to the development of interstitial chemotherapeutic methods against glioblastoma in clinic and positive results of saponin administration in experimental animals by in situ administration with interstitial injection or catheter insertion through tumor region, the chemotherapeutical potential of the marine saponins may be implemented in a form of drug products purposed for in situ administration.

Table 22. The structure and anti-glioma activity of sulfated saponins from sea cucumber Holothuria moebii [113].

\begin{tabular}{lllll}
\hline Compound and structure & \multicolumn{3}{c}{ IC50, $\mu$ M } \\
\cline { 2 - 5 } & U87-MG & U251 & SHG-44 & C6 \\
\hline
\end{tabular}

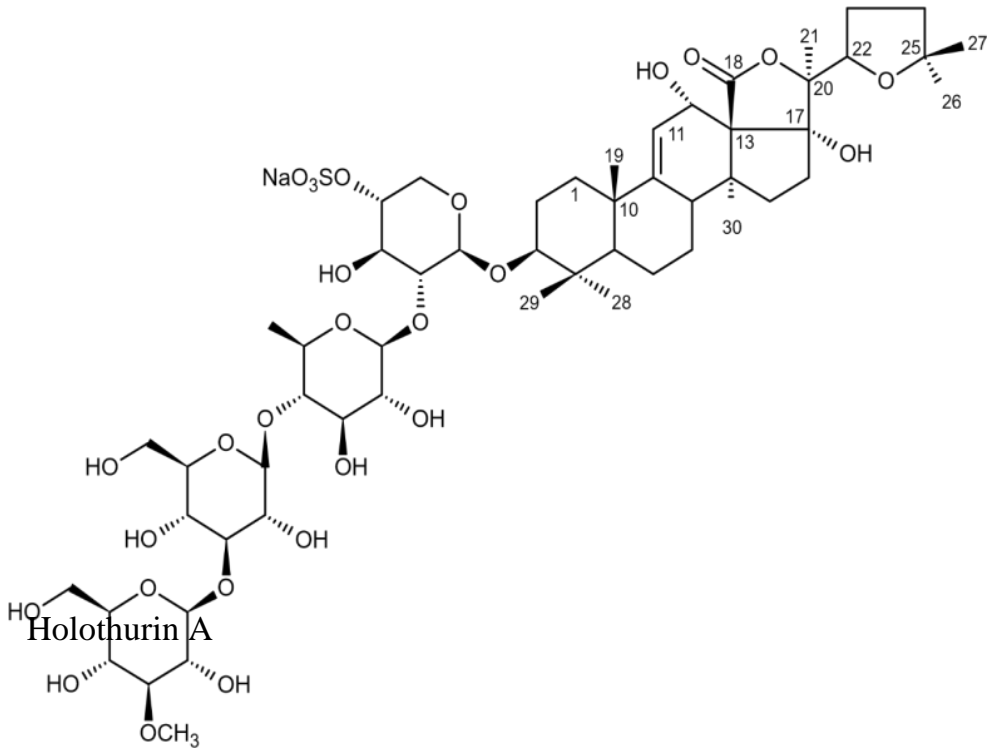


$\begin{array}{llll}4.03 \pm 0.55 & 3.76 \pm 0.08 & 3.68 \pm 0.16 & 0.99 \pm 0.17\end{array}$

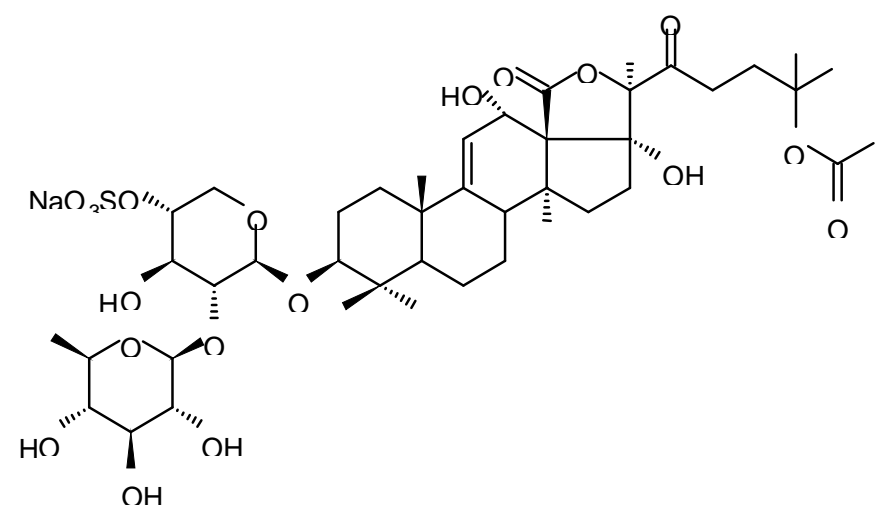

Holothurin B

$2.86 \pm 0.23$

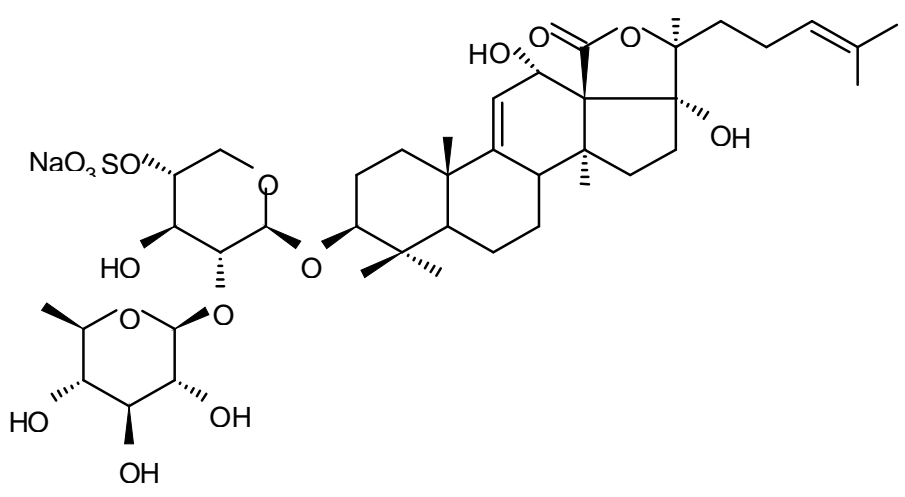

24-dehydroechinoside B

$2.72 \pm 0.04$

$6.10 \pm 0.71$

$1.99 \pm 0.18$

$2.09 \pm 0.72$

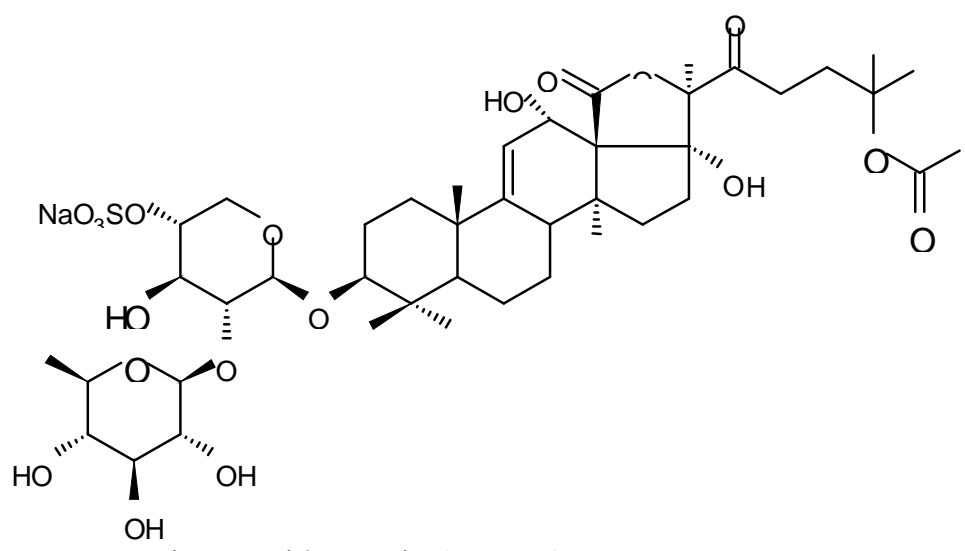

New triterpenoid saponin (see text)

$3.81 \pm 0.07$

$4.39 \pm 0.52$

$2.80 \pm 0.69$

$1.22 \pm 0.10$

Temozolomide

$>100.00$

$>100.00$

$85.8 \pm 4.10$

$69.5 \pm 6.10$

\section{Terpenes}

Terpenes are the ones of the most extensive and varied structural compounds occurring in nature displaying a wide range of pharmacological activities including antibacterial, antifungal, antiviral, anti-inflammatory, antioxidant, cytotoxic, and enzyme inhibiting influence [115].

Terpenes present a class of unsaturated hydrocarbons with general formula (C5H8)n with $n>2$. Hydrocarbon with empirical formula C5H8 (2-methyl-1,3-butadiene) is known as isoprene. Therefore, terpenes are isoprene polymers with low degree of polymerization. Living natural objects contains no isoprene polymers but rather their oxygen-substituted derivatives named terpenoids (or isoprenoids). Depending on the number of isoprene fragments, they are categorized into на monoterpenes, consisting of two terpene 
molecules $(\mathrm{C} 10 \mathrm{H} 16)$, sesquiterpenes $(\mathrm{C} 15 \mathrm{H} 24)$, diterpenes $(\mathrm{C} 20 \mathrm{H} 32)$, triterpenoids (C30H48), and tetraterpenes. Terpenoid sources include as terrestrial higher plants as marine species (bacteria, fungi, micro- and macroalgae, sponges, and others). Chemical synthesis as well as semisynthetic assembly have been playing a vital role in the terpenoid drug production [116]. Representatives of at least sesquiterpenes, di- and triterpenes was found to exert antitumor activity regarding glioma cells.

Sesquiterpenes. One of the first halogenated marine sesquiterpenes reported in the literature, aplysin was isolated from the opisthobranch gastropod mollusk Aplysia kurodai and later from the red algae belonging to genus Laurencia. Aplysin $(\mathrm{C} 15 \mathrm{H} 19 \mathrm{OBr})$ extracted from Aplysia kurodai is a bromosesquiterpene with molecular weight 295 (Figure 12). It has attracted much attention because of its potent pharmacological activities including antitumor and antioxidant effects. This compound had shown antitumor influence on the sarcoma, human breast cancer, and human gastric cancer lines via apoptosis induction $[117,118]$. Orally fed aplysin purified from the red alga Laurencia tristicha was also demonstrated to significantly suppress the growth of 7,12-dimethylbenz[a]anthracene-induced breast tumor tissues in rat models. Tumor growth inhibition activity of aplysin was suggested to related to the PI3K/AKT/FOXO3a pathway inhibition [118].

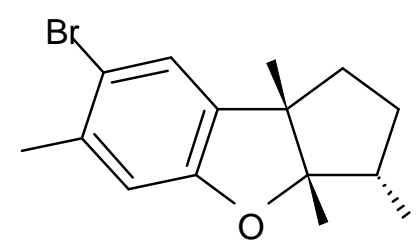

Figure 12. The structure of aplysin

Aplysin isolated from L. Tristicha and applied in concentrations 10 and $20 \mu \mathrm{g} / \mathrm{mL}$ significantly reduced cell viability in such human glioma lines as U87 MG, U251 MG, U373 MG, and M059J in a dose-dependent manner [119]. Aplysin concentrations 20, 40, and 80 $\mu \mathrm{g} / \mathrm{mL}$ significantly inhibited glioma GL26 cell proliferation rate in 24,48 , and 72 hours and this effect was found to be time- and dose-dependent. After $48 \mathrm{~h}$ treatment with aplysin, the number of GL26 cells in G0/G1 phase was significantly higher compared with the control, whereas the numbers of cells in S and G2/M phases were much lower [120]. In contrast, no significant cytotoxicity was observed in normal astrocytes as a result of the aplysin treatment within the concentration range $1-20 \mu \mathrm{g} / \mathrm{mL}$. Apoptosis detection revealed that aplysin concentrations 5 and $10 \mu \mathrm{g} / \mathrm{mL}$ can trigger apoptotic events in U-87 MG cell line in a dose-dependent fashion, with apoptosis noted in healthy astrocytes. These results confirm that aplysin selectively triggers apoptosis and cytotoxicity pathway in glioma cells. The colony-forming and transwell assays detecting the colony formation and invasion ability of cancer cells affected by drug treatment showed that the number of colonies was substantially reduced in the glioma cells treated with aplysin if compared to control. Transwell assay results demonstrated that aplysin can suppress glioma cell invasion capacity in a dose-dependent manner. Investigation of the expression profile of eighteen miRNAs related to the glioma progression revealed that miR-181, a potent tumor suppressor, was overexpressed in U-87 MG cell lines treated with $20 \mu \mathrm{g} / \mathrm{ml}$ aplysin [119]. miR181 is known to suppress the malignant properties of glioma cells by inhibiting tumor growth, inducing apoptosis, and suppressing invasive capacities. Identified miR-181 targets include Bcl-2 and MEK1 [121,122], both of which are known oncogenes that facilitate glioma progression. Aplysin significantly decreased the expression both of MEK1 and Bcl2 in a dose-dependent manner in the U-87 MG cells.

Exploration AKT and p-AKT protein expression in the glioma GL26 cells treated with aplysin showed that phosphorylated AKT expression was particularly reduced in glioma, while the total AKT level remained unchanged suggesting that aplysin may selectively inhibit PI3K/AKT signaling pathway. The level of AKT-binding heat shock protein 90 (Hsp90) was found to be decreased in the dose-dependent manner, while the total Hsp90 concentration remained unchanged suggesting that aplysin inhibits the Hsp90/AKT 
complex formation. These effects and pathway are possibly responsible for the growth inhibition of the glioma GL26 xenografts induced by aplysin with following prolonged survival period in mice with grafted tumor approximately by 1.5 and 2 times after drug administration of 40 and $80 \mu \mathrm{g} / \mathrm{kg}$ body weight, respectively [119].

Combined administration of aplysin and temozolomide was demonstrated to significantly increase glioma cell sensitivity to the effects of temozolomide if compared to single administration of temozolomide [119]. Taking to consideration the earlier received information about miR-181 enhancing glioma cell sensitization to temozolomide by silencing MEK1 expression [122], it may be suggested that aplysin-induced temozolomide sensitivity may be also dependent on MEK1 pathway in glioma cells.

Bcl-2 was also found to be downregulated in glioma cells treated with aplysin, implying that this antiapoptotic protein may be also involved in its effect on the action of temozolomide on glioma cells. Therefore, aplysin can suppress malignant properties of glioma and enhance anti-tumor efficacy of temozolomide making him a prospective lead compound for development of the potent clinical anti-glioma drugs.

Diterpenes. Fourteen compounds namely eupalmerin acetate, isoeupalmerin acetate, 3'-O-acetyl-pseudopterosin U, 3-epi-14-deoxycrassin, asperdicin, pseudoplexauric acid methyl ester, 2-deoxyasperdiol acetate, $\mathrm{N}$-methylpyridinum-3-sulfonate, dinosterol, kallolide A, kallolide A acetate, asperdoil, asperdoil acetate, isoasperdoil isolated from the combined terpenoid-rich fraction of the chloroform-methanol extract of specimens Caribbean gorgonian octocoral Eunicea succinea, and one compound, placotrid $\mathrm{O}$ isolated from the marine sponge Plakortis halichondroides were tested on the malignant human glioma cell lines U87-MG and U373-MG for the presence of antitumor activity [123]. Placotrid O was the most potent one among the compounds tested in the study, but its cytotoxicity was substantially lowered after it was stored in solution for a few weeks. IC50 value changed from 4.0 to $31.0 \mathrm{mkmol} / \mathrm{l}$ in the U87-MG cells and from 4.0 to $15.4 \mathrm{mkmol} / \mathrm{l}$ in the U373-MG cells.

Table 23. The structure and anti-glioma activities of plakortide O isolated from the marine sponge Plakortis halichondroides [123].

\begin{tabular}{|c|c|c|c|}
\hline \multirow[t]{2}{*}{ Compound } & \multirow[t]{2}{*}{ Structure } & \multicolumn{2}{|c|}{$\mathrm{IC}_{50,} \mu \mathrm{mol} / \mathrm{L}$} \\
\hline & & U87-MG & U373-MG \\
\hline Plakortide O & & 4.0 & 4.0 \\
\hline
\end{tabular}

Five compounds exerted inhibiting effects on the cell viability in both glioma cell lines with IC50 ranging from 5.1 to $64.2 \mu \mathrm{mol} / \mathrm{L}$ (Table 24). The most potent compound among them was eupalmerin acetate belonging to the cembranolide diterpenes. It was stable for several months with IC50 values $5.1 \mu \mathrm{mol} / \mathrm{L}$ for U87-MG cells and 6,9 $\mathrm{mol} / \mathrm{L}$ for U373-MG cells. This range of IC50 is comparable with that of cisplatin, which is in the ranging from 5 to $10 \mu \mathrm{mol} / \mathrm{L}$ for these cell types. Two compounds (pseudoplexauric acid methyl ester and 2-deoxyasperdiol acetate) exerted inhibiting effects against only one cell line in concentration below $100 \mu \mathrm{mol} / \mathrm{L}$. The other compounds had IC50 values greater than $100 \mu \mathrm{mol} / \mathrm{L}$ in both cell lines and were therefore considered not potent enough to serve as anticancer drugs for these cell types.

Further investigations were focused on anti-glioma mechanisms involved into eupalmerin acetate activity. It was found out that eupalmerin acetate induces G2-M arrest and apoptosis in cells of both U87-MG and U373-MG lines, at least, partly activating the mitochondrial pathway and inducing Bax translocation to the mitochondria, as well as via activation of the c-Jun NH2-terminal kinase (JNK) pathway. Eupalmerin acetate in the experiments with nude mice with xenografted tumor nodes obtained from U87-MG cells 
have demonstrated significant antitumor effects. Authors concluded that this result indicate eupalmerin acetate provides therapeutic efficacy against malignant glioma cells and it as well as similar marine-based compound may hold promise as a clinical anticancer agent [123].

Table 24. The structure and anti-glioma activities of diterpenes from the gorgonian octocoral Eunicea succinea [123].

\begin{tabular}{lll}
\hline Compound & Structure & IC50 $(\mu \mathrm{mol} / \mathrm{L})$ \\
Eupalmerin acetate & U373-MG \\
\hline
\end{tabular}

Isoeupalmerin acetate<smiles>C=C(CC/C=C\CCC[C@@H](C)C(=O)OC(=O)O)[C@H]1OC(=O)/C(=C/C)C1C</smiles>

3'-O-acetylpseudopterosin U

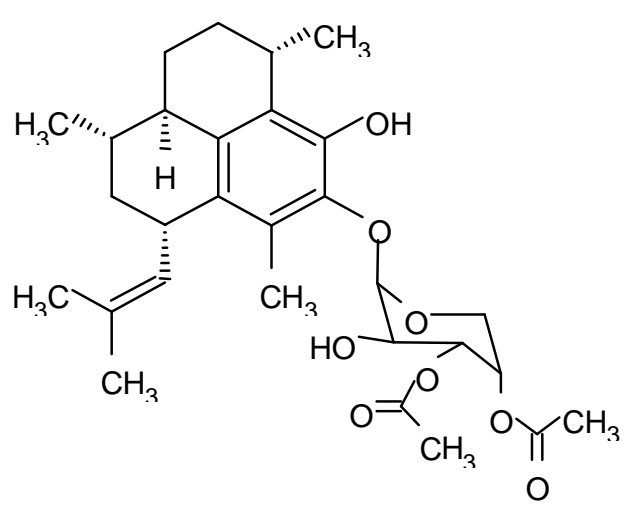

3-Epi-14-deoxycrassin
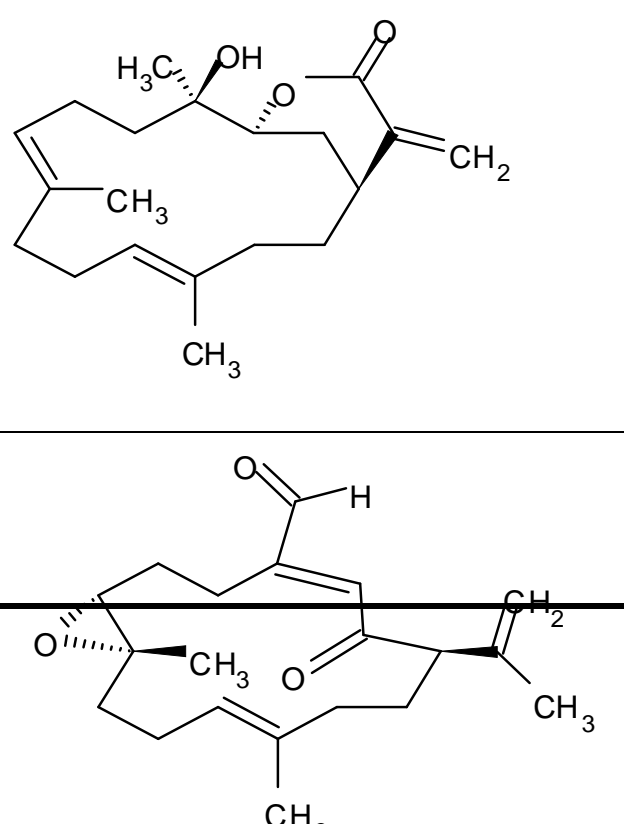
Triterpenes. An isomalabaricane-type triterpene, stellettin B, isolated from the marine sponge Jaspis stellifera was experimentally tested on 39 human cancer cell lines including lung, gastric, ovarian, breast, kidney, prostate, and colon cancer lines, melanoma as well as gliomas (U251, SF-295, SF-539, SF-268, SNB-75 и SNB-78). Human glioblastoma cell SF295 exhibited high sensitivity to stellettin B (Table 25). At the same time, stellettin $B$ have shown very weak inhibiting activity towards normal cell lines such as human mammary epithelial cells (HMEC), human renal tubule epithelial cells (RPTEC), normal human bronchial epithelial cells (NHBE), normal human prostate epithelial cells (PrEC) with GI50 higher than $10 \mu \mathrm{M}$ suggesting its relatively selective cytotoxicity against glioma cells compared to normal human cell lines. Stellettin B treatment of the cell lines induced apoptosis in a dose-dependent manner as well as enhanced caspase 3/7 activity, cleavage of poly-(ADP-ribose) polymerase (PARP) and reactive oxygen species (ROS) production in SF295 cells. Each of these pathways is known to be involved in apoptosis.

Effect of triterpene on the phosphorylation of PI3K/Akt and RAS/MAPK pathways signal proteins including Akt, p38 and ERK, was examined for elucidation of the molecular mechanisms responsible for inhibition of the glioma cell proliferation and apoptosis induction. Stellettin B strongly inhibited Akt phosphorylation but did not affect p-ERK and p-p38 activity and did not inhibited PI3K activity in concentration below $1 \mu \mathrm{M}$ suggesting that antiproliferative and apoptosis-inducing activity is not attributed to PI3K inhibition [124]. The direct target of stellettin B might be signal protein upstream of Akt other than PI3K. Authors have suggested that stellettin B might be a lead compound for discovery of perspective drug candidate for treatment of a part of glioblastomas.

Table 25. In vitro anti-glioma activity of stellettin B from marine sponge Jaspis stellifera against human glioblastoma cell line SF295 [124].

\begin{tabular}{l|l|l|l|}
\hline Compound & Structure & IC50 \\
\hline Stellettin B & & & \\
\hline $\mathrm{IG}_{50}\left(\mathrm{IC}_{50}\right)$ & &
\end{tabular}

\section{Peptides, cyclopeptides}

Natural or synthetic compounds composed of the molecules presented with $\alpha$-amino acid residues and linked into a chain via peptide (amide) bonds make a polypeptide class or family. Cyclic peptides make a group of compounds built with proteinogenic $\alpha$-amino 
acids in accordance to the principles, which is similar to the one of linear polypeptides. Thus, they have the specific amide (peptide) bonds but in contrast to the regular proteins, they contain D- configuration $\alpha$-amino acids and modified L- $\alpha$-amino acids looped into macrocycles and included into peptide chains. Their sources are generally presented with mushrooms and microorganisms. Cyclic depsipeptides are cyclopeptides with one or several amino acids replaced with oxy acids, and therefore, their molecules apart from amide groups contain one or few ester groups. Cyclopeptide list includes many well-known antibiotics and antitumor agents [125].

Peptides. In 1988 Nakamura et al. isolated a peptide named tachyplesin I from the hemocytes of the horseshoe crab Tachypleus tridentatus, determined its chemical structure, and demonstrated antitumor properties. Later this peptide was synthesized by Hanyu Bioengineering Company (China). Tachyplesin I presents cationic peptide with 17 residues (NH2-K-W-C-F-R-V-C-Y-R-G-I-C-Y-I-R-R-C-R-CONH2) [126]. In addition to antimicrobial activity tachyplesin I can inhibit proliferation of such tumor cells as human hepatocellular carcinoma SMMC-7721 and TSU prostate cancer cells [127]. Tachyplesin I has also been demonstrated to activate classic complement pathways regulating tumor cell lysis and altering tumor suppressor genes expression and oncogenes to induce cell differentiation and reverse the malignant phenotype [128]. Negatively charged components of cancer cells, which are quite different from neutral normal cells, are more vulnerable by the positively charged cationic peptides, including tachyplesin I. Electrostatic attraction between cancer cell and cationic peptides is thought to play a major role in the selective disruption of cancer cell membranes helping avoid development if the typical drug resistance mechanism [129].

Tachyplesin I applied in concentrations 10,40 and $80 \mu \mathrm{g} / \mathrm{mL}$ was shown in the in vitro experiments to inhibit the viability and proliferation of gliomaspheres isolated from U251 glioma cell lines in dose-dependent manner due to the plasma membrane damage and indued differentiation of the glioma stem cells [130]. Proteomic analysis using twodimension difference gel electrophoresis and stable isotope dimethyl labeling based liquid chromatography-mass spectrometry/mass spectrometry revealed that in response to tachyplesin I influence 192 proteins were differentially expressed in U251 gliomaspheres [131]. Major part of those proteins is involved into the cellular metabolism processes, in particular, glycolysis processes, and many proteins are identified as cytoskeleton proteins and lysosomal acid hydrolases. The glycolytic/gluconeogenesis enzymes including alphaenolase (ENO1), gamma-enolase (ENO2), triosephosphate isomerase (TPI1) and phosphoglycerate kinase 1 (PGK1) were down-regulated in response to tachyplesin I treatment indicating that tachyplesin I may disrupt normal energy metabolism in gliomaspheres via reduced glycolysis.

U251 gliomaspheres treatment with Tachyplesin I altered the expression of 18 cytoskeleton proteins. Two of those proteins, namely vimentin and ezrin are known to be involved into the metastasis regulation and down-regulated due to the treatment with tachyplesin I suggesting that cytoskeleton is affected by tachyplesin I as well. Apart of that, tachyplesin I significantly reduced levels of lysosomal protease enzymes, cathepsins B and $\mathrm{D}$, in gliomaspheres that are known to be involved into autophagy and apoptosis pathways. Inhibition of cathepsins B or D activity attenuates extracellular matrix degradation thus reduces migration of glioma cells [132]. Those results suggest that tachyplesin I has a potency as a therapeutic agent for glioma by targeting the lysosomal activity. Protein-protein interaction network analysis of differentially expressed proteins showed that DNA topoisomerase 2-alpha (TOP2A) mar serve as a possible critical target protein of tachyplesin I. DNA damage and fragmentation induced by covalent binding of TOP2A to DNA as well as forced expression of TOP2A in cells are known to trigger the apoptotic cell death. Furthermore, TOP2A level is known to have close relationship with the potency of anti-tumor drugs and high level of TOP2A regulating the main pathway of drug susceptibility. And vice versa, decreased level of TOP2A mutation can induce the loss of the anti-tumor drug targeting and multiple drug resistance development. Elevated TOP2Alevel was determined in the U251 gliomaspheres treated with tachyplesin I 
suggesting possible synergistic effect of tachyplesin I and anticancer drugs targeting TOP2A. Such combination may enhance antiglioma efficiency of chemotherapy [131].

Cyclodepsipeptides. Marine cyanobacteria have continued to be a prolific source of cytotoxic depsipeptides applicable to cancer research. One of notable representatives from the cyanobacterial cyclic peptide group is a natural cyclopeptide product coibamide A that was initially isolated from marine filamentous cyanobacterium Leptolyngbya sp. and named after its geographical location as it was collected from the Coiba Island National Park in Panama. Coibamide A is a lariat depsipeptide that features a highly methylated 22-membered macrocycle with a pseudo-tetrameric side chain (Table 26). The coibamide A in vitro screening tests performed in the National Cancer Institute with 60 human tumor cell lines using anticancer drug screen assay revealed differential picomolar-nanomolar potency as a tumor growth inhibitor against many cell lines and some histological selectivity for several malignant cell types such as CNS-derived tumors, breast, ovarian and colon cancer cells [133].

Coibamide A induces concentration- and time-dependent cell death in human U87MG and SF-295 glioblastoma cells. 72-hours long influence of coibamide A on the cells from both lines demonstrated reduced proliferation and substantial morphological alterations. At the same time, coibamide A induced cytotoxicity appeared only for nanomolar depsipeptide concentrations (table 26). Cyclization of the depsipeptide is likely to be a critical determinant of the cellular response to coibamide A because two full-length linearized analogues namely coibamide dehydrated seco acid and coibamide seco acid did not exert cytotoxicity towards cells of both glioblastoma and neuroblastoma [134].

Table 26. The structure and cytotoxicity of the coibamide A from the marine cyanobacterium Leptolyngbya sp. against glioma cells [134].

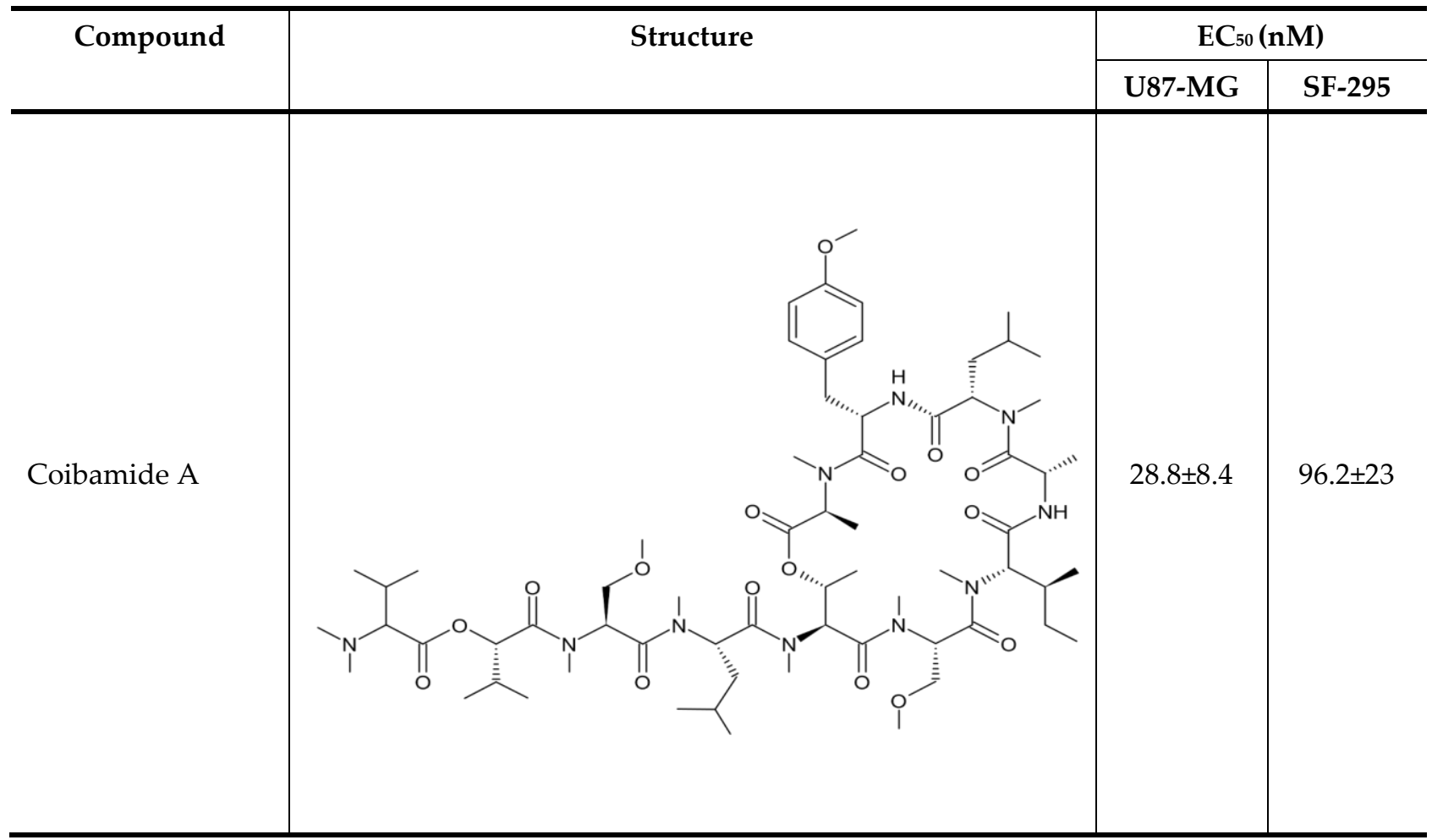

Morphological and biochemical cell death forms induced by coibamide A wee different in accordance to the cell types. Glioblastoma SF-295 cells demonstrated caspase-3 activation whereas cell death in glioblastima U87-MG line was characterized with a vast cytoplasmatic vacuolization without obvious apoptotic peculiarities despite these cells were approximately three times more sensitive to coibamide A than Sf-295 cells. 
Therefore, coibamide A can induce apoptosis in SF-295 cells via activation of a classic caspase-3-dependent pathway and also can effectively trigger cell death in U87-MG glioma cells via an alternate (non-apoptotic) pathway when caspase activity is inhibited. It was shown with the use of biochemical and morphological autophagia criteria that coibamide A induces accumulation of autophagosomes in apoptosis resistant U-87-MG cells i.e. induced autophagia in those cells. It should be mentioned that coibamide A induced autophagosome storage in the human glioblastoma cells via mTOR-independent mechanism. No change was observed in the phosphorylation state of ULK1 (Ser-757), p70 S6K1 (Thr-389), S6 ribosomal protein (Ser-235/236) и 4EBP-1 (Thr-37/46) [134].

Both cell types of glioblastomas U87-MG and SF-295 in the in vitro tests have shown significant increase of the G1-phase cell number in response to the effects of coibamide A and appropriate reduction of the S-phase cells [135]. Therefore, a coibamide A action is phase specific and coibamide $A$ induces a cell cycle block at the G1/S phase transition. In addition, coibamide A reduced migration and invasive capacity of U87-MG and SF-295 cells, inhibited extracellular VEGFA secreted from glioblastoma U87-MG cells, attenuated proliferation and migration of normal human umbilical vein endothelial cells (HUVECs) and selectively decreased expression of vascular endothelial growth factor receptor 2 (VEGFR2) with low $\mathrm{nM}$ potency. All these observations suggest the presence of antiangiogenic activity of coibamide A. Intratumor administration of $300 \mu \mathrm{g} / \mathrm{kg}$ coibamide A in nude mice with xenograft glioblastoma model inhibited a growth of subcutaneous xenograft U87-MG tumor. Tumor volume in all animals treated with coibamide A remained ta initial unchanged level with no significant growth for more than 4 weeks of experimental therapy [135].

It is well known that U87-MG cell lack the PTEN signaling pathway and have normal functioning of the p53 signaling pathway whereas as SF-295 cells are lacking both p53 and PTEN. Mutations of these two genes are frequently found in GBM; analysis of 601 genes obtained from 91 stratified patients with GBM executed in the frames of the "The Cancer Genome Atlas" project showed that the frequency of the p53 and PTEN mutations is $42 \%$ $33 \%$, respectively [136]. Coibamide A exerted cytotoxic influence on six glioma cell lines and four of them (SF-295, U251, SF-539, SNB-19) were characterized with the lack of both signaling pathways PTEN and p53. Two of these lines (SNB-75, SF-268) have disorders of the p53 pathways but normally functioning PTEN pathway. Therefore, coibamide A may be considered as effective glioma cellular toxin regardless of the p53 and PTEN status. This specific capacity of coibamide A to induce more than one way killing cancer cells is thought to be especially helpful pharmacological property of the antitumor therapy.

Therefore, coibamide $\mathrm{A}$ is one of the most potent inhibitors of the human glioblastoma cells with high drug potential. Despite receptor coibamide A structure is not determined, it should be considered as a realistic clinical candidate.

The known cyclodepsipeptide valinomycin and two new cyclodepsipeptides named as streptodepsipeptide P11A and streptodepsipeptide P11B were isolated from a culture of the marine actinomycete Streptomyces sp. P11-23B, which was obtained from the marine mud sample [137]. Valinomycin was previously isolated from several Streptomyces species and is well known to enhance the $\mathrm{K}+$ permeability of several membrane systems including mitochondria, erythrocytes, and lipid bilayers. This cyclodepsipeptide was also reported to be active against tumors, bacteria, fungi and severe acute respiratory-syndrome coronavirus. Valinomycin is composed of four units of D-valine (DVal), L-valine (L-Val), D- $\alpha$-hydroxyisovaleric acid (D-Hiv), and L-lactate (L-Lac) with a trimer structure of cyclo-(D-Val-L-Lac-L-Val-D-Hiv)3. Streptodepsipeptide P11A had the molecular formula of C53H88N6O18. Its structure was determined as cyclo-(D-Val-L-Lac-L-Val-D-HivD-Val-L-Lac-L-Val-D-Hiv-D-Val-L- Lac-L-Val-D-Hba). Streptodepsipeptide P11B had the molecular formula of C52H86N6O18 and the structure was elucidated as cyclo-(D-Val-LLac-L-Val-D- Hiv-D-Val-L-Lac-L-Val-D-Hiv-D-Val-L-Lac-LVal-L-Lac).

Three isolated cyclodepsipeptides were assayed for their activity against the proliferation of four different glioma cell lines (U251, U87-MG, SHG-44, and C6). The results showed that streptodepsipeptides P11A and P11B had potent activity with IC50 values of 
0.3-0.4 $\mu \mathrm{M}$ and 0.1-1.4 $\mu \mathrm{M}$, respectively (table 27), while valinomycin showed much stronger antiproliferative activity with IC50 values ranging from 7.6 to $30.0 \mathrm{nM}$. The doxorubicin had activity with IC50 0.4-3.3 $\mu \mathrm{M}$. Two new compounds were also tested for growth inhibiting activity in normal human astrocytes, and IC50 values were approximately 9.1 $\mu \mathrm{M}$ for P11A and 3.5 $\mu \mathrm{M}$ for P11B. The ratios of IC50 for human astrocytes to IC50 for glioma cells were within the range of 23-30 for streptodepsipeptide P11A and 335 for streptodepsipeptide P11B. Streptodepsipeptide P11A $(0.8 \mu \mathrm{M})$ blocked U87-MG cell cycle at the G0/G1 phase; cell population at the G0/G1 phase was significantly increased by $40.92 \%$ after 12 hours long treatment in comparison to negative control. Doxorubicin $(0.8 \mu \mathrm{M})$ as a positive control also had $49.69 \%$ DNA increase at G0/G1 phase. Similar result was also obtained from streptodepsipeptide P11A treated U251 cells. After $72 \mathrm{~h}$ treatment streptodepsipeptide P11A $(0.8 \mu \mathrm{M})$ caused $20.40 \%$ increase in total number of apoptotic cells (early and late apoptotic cells) compared to the control. Moreover, streptodepsipeptide P11A ( $5 \mu \mathrm{M}$ or $10 \mu \mathrm{M}$ for $48 \mathrm{~h}$ ) significantly downregulated expression of hexokinase 2 (HK2), 6-phosphofructo-2-kinase/fructose-2,6-bisphosphatase (PFKFB3), glutaminase (GLS), and fatty acid synthase (FASN) and slightly down-regulated of pyruvate kinase M2 (PKM2). Valinomycin remarkably reduced HK2, FANS, GLS expression levels and slightly affected regulation of PKM2 [137]. These data suggest that targeting multiple tumor metabolic regulators might be an antiglioma mechanism of streptodepsipeptides.

Table 27. The structure and anti-glioma activity of cyclodepsipeptides from actinomycete Streptomyces sp. P11-23B [137].

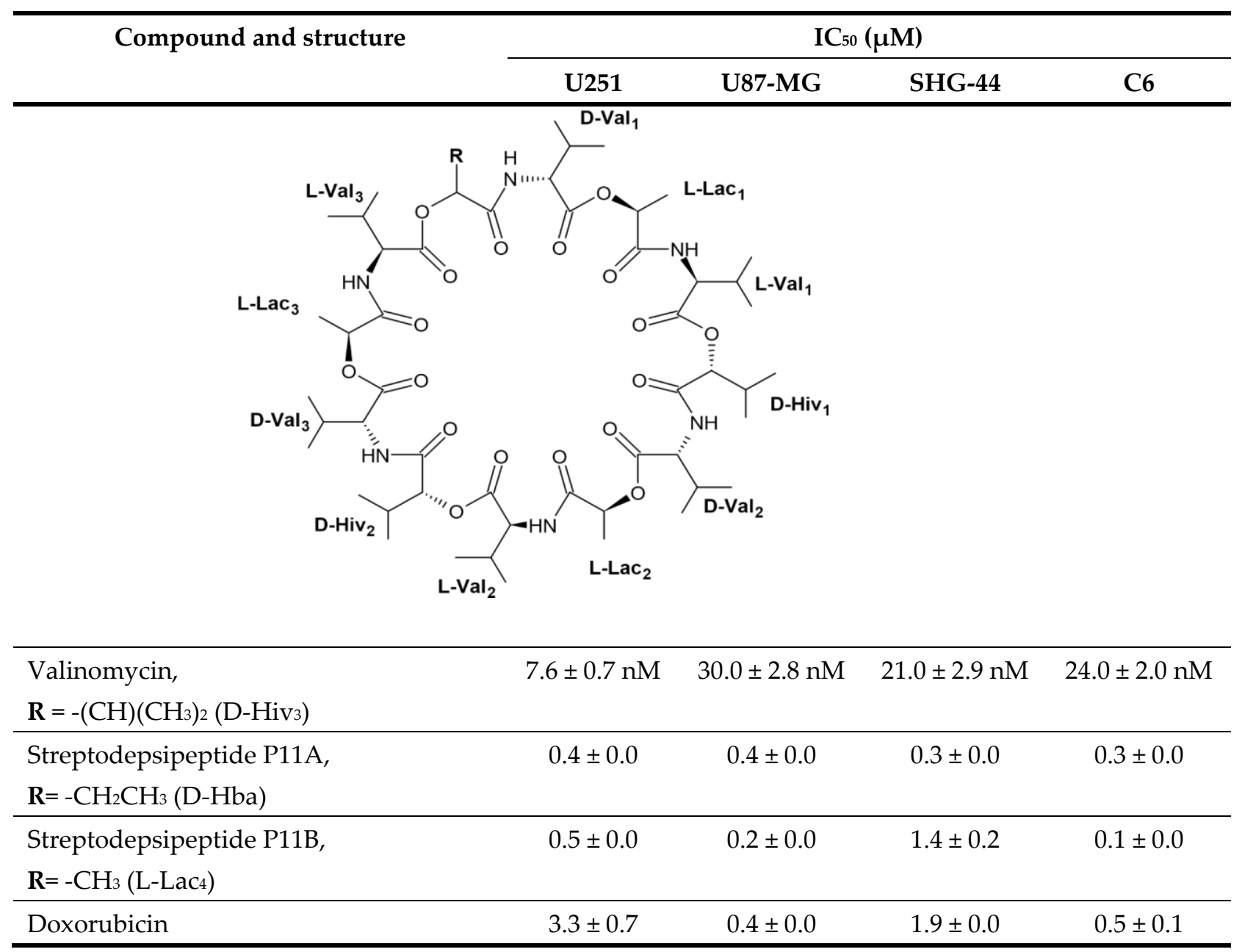

\section{Steroids and ergosterols}


Steroids. Steroids are organic compounds built in natural sources from isoprenoid precursors. Specific structural steroid pattern is the presence of condensed tetracyclic gonan system (former name is steran or cyclopentanoperhydrophenanthrenes) composed of three cyclohexane rings in non-linear joint and one cyclopentane ring. Gonan core may be saturated or partially unsaturated and contain alkyl groups and some functional groups including hydroxyl, carbonyl, or carboxyl. Steroids possess a variety of pharmacological effects including antitumor activity [138].

Five structurally close steroid compounds namely $22 \mathrm{E}, 24 \xi)-26,27-$ bisnor-24-methyl$5 \alpha$-cholest22-en-3 $\beta, 5,6 \beta, 15 \alpha, 25$-pentol 25-O-sulfate (the molecular formula C26H43O8S ${ }^{-}$), (22E,24R,25R)-24-methyl-5 $\alpha$-cholest-22-en-3 $\beta, 5,6 \beta, 15 \alpha, 25,26$-hexol 26-O-sulfate, (28R)-24-

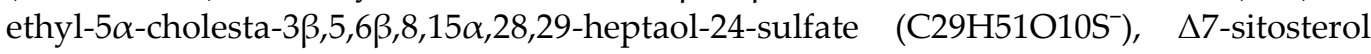
and (25S)- $5 \alpha$-cholestane- $3 \beta, 5,6 \beta, 15 \alpha, 16 \beta, 26$-hexaol (C28H49O8) were isolated from the methanol extract of the starfish Ctenodiscus crispatus and investigated for their antiglioma activity [139]. Only the latter compound, which structure is presented on figure 13 , have shown significant dose-dependent cytotoxicity in a range of concentrations 50, 100 , and $200 \mu \mathrm{M}$ against glioblastoma U87MG cells via inhibition of cell growth and apoptosis induction.

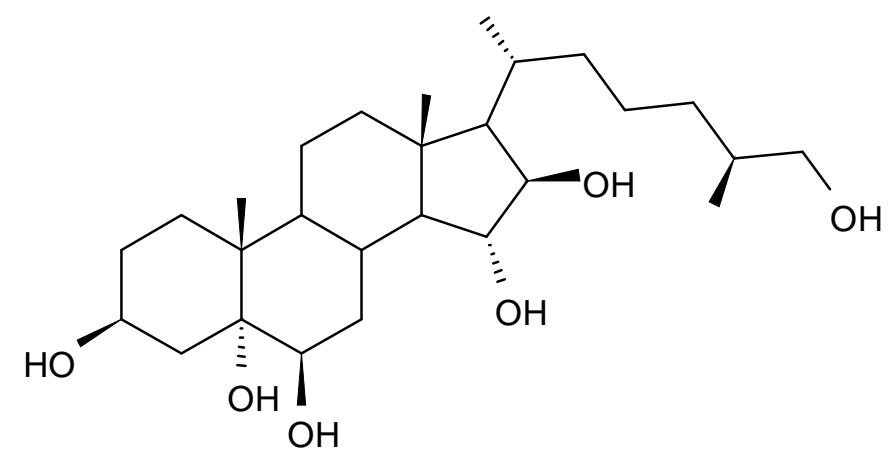

Figure 13. The structure of the steroid with anti-glioblastoma U87MG activity isolated from starfish

Ctenodiscus crispatus [139].

After the treatment with anti-glioma steroid, the expression of Bcl-2 proteins was dose-dependently decreased whereas expression of Bax protein was accumulated in increasing concentrations. The ratio of Bax/Bcl-2 exhibited a dose-dependent increase in the U87MG cells. The treatment with this steroid significantly increased the cleavage of caspase-3, caspase-9 and poly (ADP-ribose) polymerase (PARP) in a dose-dependent manner [139]. Thus, anti-glioblastoma effects of active steroids were mediated through the apoptotic process.

Ergosterols. Ergosterols are a class of compounds with tetracyclic skeleton, short alkyl chain, and many hydroxyl groups. They are the important biomembrane component involved in numerous biological functions and playing significant role in the membrane fluidity regulation, cellular cycle control, and organizing membranes for signal transduction and protein trafficking. Ergosterols are usually found in fungi, marine sponges, corals, and in bacteria [140]. They have a capacity to produce clinically useful compounds with different structures, such as streptomycins, actinomycins, adriamycin, and vancomycin and exert antifungal, immunosuppressant, and anticancer properties.

Three new ergosterols, ananstreps A, B, and C, and ten previously discovered ones (Figure 14), were isolated from the culture broth of marine Streptomyces anandii H41-59 and tested for their cytotoxicity against human glioblastoma cell line SF-268. New ergosterols was defined as ergosta-7,22-diene- $3 \beta, 5 \alpha, 6 \beta, 27$-tetraol (ananstrep A, a molecular formula C28H46O4), ergosta-7,22-diene-3 $\beta, 5 \alpha, 6 \beta, 9 \alpha, 25$-pentol (ananstrep B, a molecular formula $\mathrm{C} 28 \mathrm{H} 46 \mathrm{O} 5$ ) and $5 \beta, 6 \beta$-epoxy-ergosta-7,22-diene-3 $\beta, 7 \beta$-diol (ananstrep $\mathrm{C}$, a molecular formula $\mathrm{C} 28 \mathrm{H} 44 \mathrm{NaO} 3$ ). The rest of compounds were identified as ergosta-7,22-diene- 
$3 \beta, 5 \alpha, 6 \beta, 25$-tetraol (4), ergosta-7,22-diene-3 $\beta, 5 \alpha, 6 \beta$-triol (5), ergosta-7,22-diene-3 $\beta, 5 \alpha, 6 \alpha$ triol (6), ergosta-7,22-diene-3 $\beta, 5 \alpha, 6 \beta, 9 \alpha$-tetraol (7), $5 \alpha, 6 \alpha$-epoxy-ergosta-8(9),22-diene$3 \beta, 7 \alpha$-diol (8), $5 \alpha, 6 \alpha$-epoxy-ergosta-8(14),22-diene-3 $\beta, 7 \alpha$-diol (9), ergosta-8(9),22-diene$3 \beta, 5 \alpha, 6 \beta, 7 \alpha$-tetraol (10), ergosta- $8(14), 22$-diene-3 $3,5 \alpha, 6 \beta, 7 \alpha$-tetraol (11), ergosta-5,7,22triene- $3 \beta$-ol (12) and 5 $5,6 \beta$-epoxy-ergosta- $8(14), 22$-diene- $3 \beta, 7 \beta$-diol (13). Major part of already described compounds were isolated from fungi and sponges.

All isolated compounds showed some moderate cytotoxicity against SF-268 cells, whereas $\Delta 8(9)$-sterols and $\Delta 8(14)$-sterols, namely, ananstrep C, compounds 8,10 and 11 displayed higher efficacies against glioblastoma cells with IC50 (Table 28) [141]. Active compounds showed cytostatic activity, that was not lower than that of cisplatin belonging to chemotherapeutics. Attention should be given to ananstrep $C$ that possess a $\Delta 8(9)$ moiety and a rare $\beta$-orientation at C-5 and C- 6 , which are commonly $\alpha$-orientation in this kind of compounds, indicated much better effect against SF-268.

Eleven sterols were isolated from sea anemone Anthopleura midori and then identified. First six (1-6) compounds presented rare polyoxygenated ergosterols with a 24,28epoxy moiety. These six compounds were three pairs of 24 isomers of ergosterols with a 24,28-epoxy group. Epoxyergosterols 1 and 2 were found to be new natural products and 3-6 were new compounds. Compounds 1 and 2 were C-24 isomers of ergosterols with an epoxy moiety at C-24 and C-28 and had the same molecular formula $\mathrm{C} 28 \mathrm{H} 46 \mathrm{O} 2$. The structures of 1 and 2 were assigned as 24(R),28-epoxyergost-5-en-3 $\beta$-ol and 24(S),28-epoxyergost-5-en-3 $\beta$-ol, respectively. Compounds 3 and 4 were also C-24 isomers of 24,28 epoxyergosterols and their structures were elucidated as 24(R),28-epoxyergost-3-one$5 \alpha, 6 \alpha$-diol (3) and 24(S),28- epoxyergost-3-one- $5 \alpha, 6 \alpha$-diol (4). Compounds 5 and 6 were also a pair of C-24 isomers with molecular $\mathrm{C} 30 \mathrm{H} 50 \mathrm{O} 5$ and structures $24(\mathrm{R}), 28$-epoxyergost-3-acetyl-3 $\beta, 5 \alpha, 6 \alpha$-triol and $24(S), 28$ - epoxyergost-3-acetyl-3 $\beta, 5 \alpha, 6 \alpha$-triol, respectively (Figure 15). The rest of the previously known substances were identified as $5 \alpha, 8 \alpha$ epidioxyergosta-6,24(28)-dien-3 $\beta$-ol (7), cholestane-3 $\beta, 5 \alpha, 6 \alpha$-triol (8), holest-5-en-3 $\beta$-ol (9), ergosta-5,24(28)-dien-3 $\beta$-ol (10), and ergosta-5,22,24(28)-trien-3 $\beta$-ol (11).

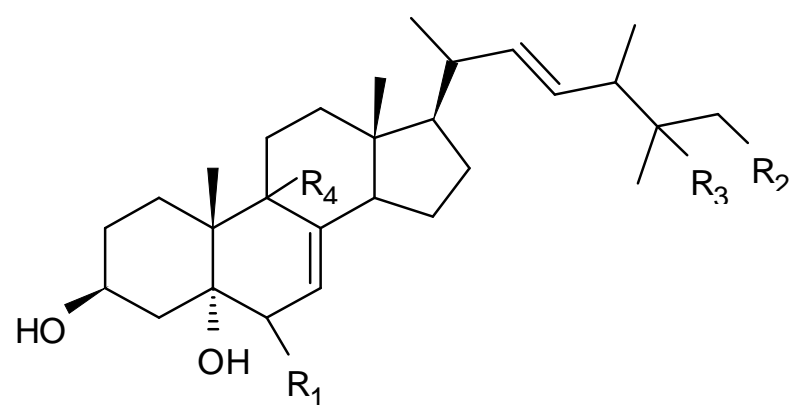

$1 \mathrm{R}_{1}=\beta-\mathrm{OH}, \mathrm{R}_{2}=\mathrm{OH}, \mathrm{R}_{3}=\mathrm{H}, \mathrm{R}_{4}=\mathrm{H}$

$2 \mathrm{R}_{1}=\beta-\mathrm{OH}, \mathrm{R}_{2}=\mathrm{H}, \mathrm{R}_{3}=\mathrm{OH}, \mathrm{R}_{4}=\mathrm{OH}$

$4 \mathrm{R}_{1}=\beta-\mathrm{OH}, \mathrm{R}_{2}=\mathrm{H}, \mathrm{R}_{3}=\mathrm{OH}, \mathrm{R}_{4}=\mathrm{H}$

$5 \mathrm{R}_{1}=\beta-\mathrm{OH}, \mathrm{R}_{2}=\mathrm{H}, \mathrm{R}_{3}=\mathrm{H}, \mathrm{R}_{4}=\mathrm{H}$

$6 \mathrm{R}_{1}=\alpha-\mathrm{OH}, \mathrm{R}_{2}=\mathrm{H}, \mathrm{R}_{3}=\mathrm{H}, \mathrm{R}_{4}=\mathrm{H}$

$7 \mathrm{R}_{1}=\beta-\mathrm{OH}, \mathrm{R}_{2}=\mathrm{H}, \mathrm{R}_{3}=\mathrm{H}, \mathrm{R}_{4}=\alpha-\mathrm{OH}$

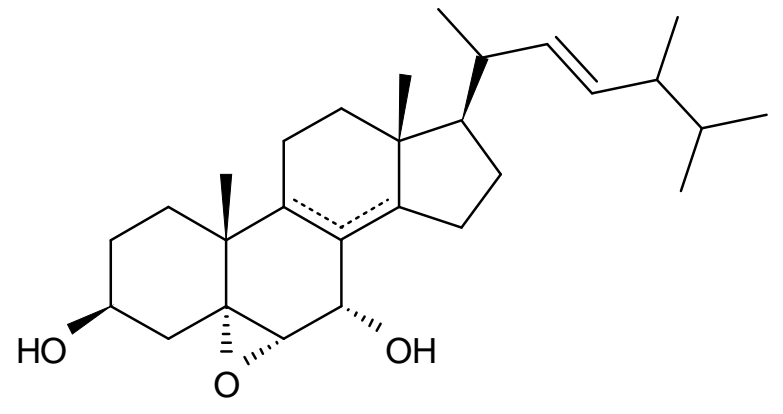

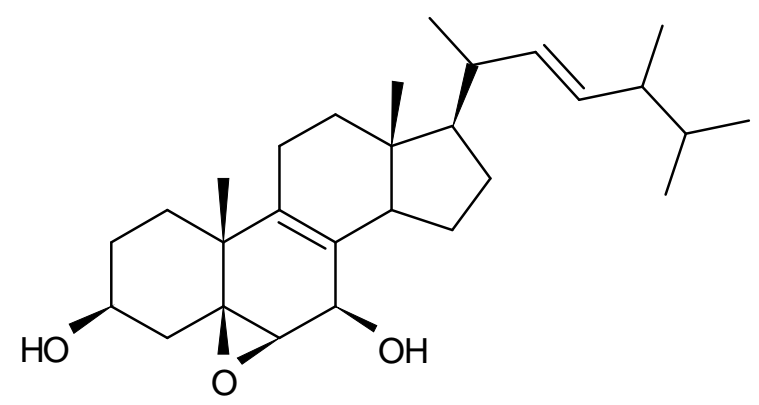

3

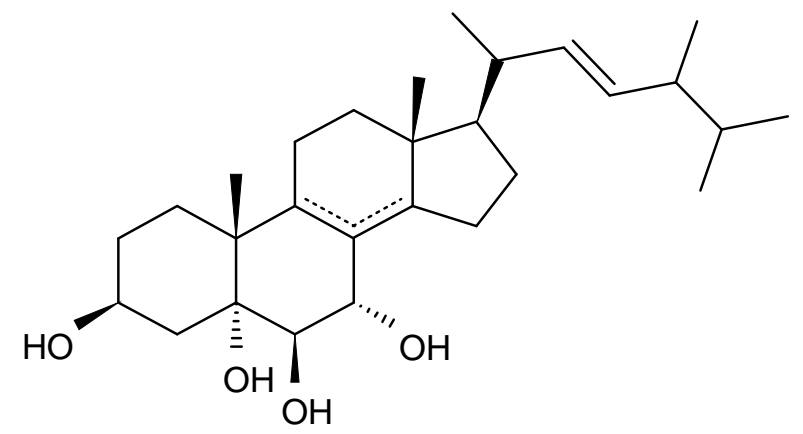




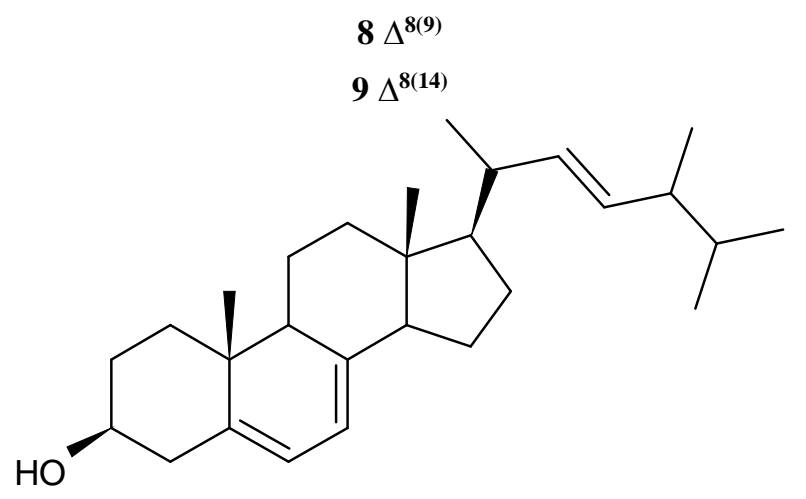

12

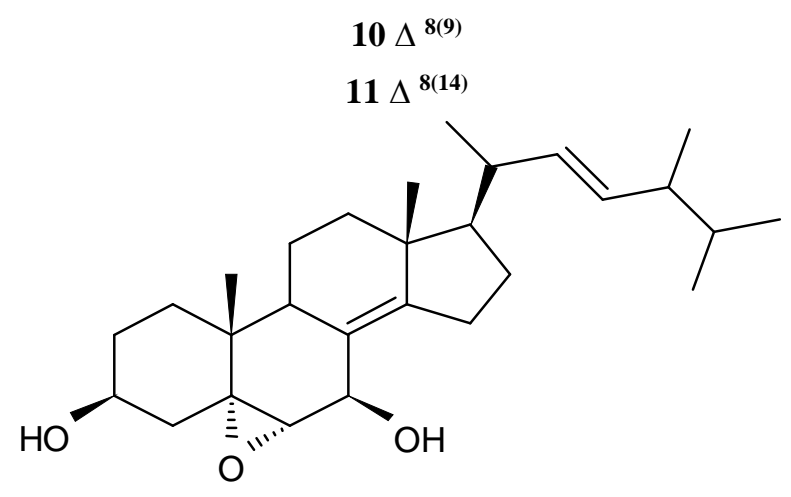

13

Figure 14. Structures of ergosterols isolated from the culture broth of marine Streptomyces anandii H41-59 (reproduced from work 141).

Table 28. Cytotoxic activity of ergosterols from the marine actinomycete Streptomyces anandii H41-59 against human glioblastoma SF-268 cells [141].

\begin{tabular}{|c|c|c|c|}
\hline Compound & $\mathrm{IC}_{50}(\mu \mathrm{g} / \mathrm{mL})$ & Compound & $\mathrm{IC}_{50}(\mu \mathrm{g} / \mathrm{mL})$ \\
\hline ananstrep A & $>50$ & 8 & 15.5 \\
\hline ananstrep B & $>50$ & 9 & $>50$ \\
\hline ananstrep C & 13.0 & 10 & 27.8 \\
\hline $4^{*}$ & $>50$ & 11 & 25.1 \\
\hline 5 & $>50$ & 12 & $>50$ \\
\hline 6 & $>50$ & 13 & $>50$ \\
\hline 7 & $>50$ & cis-dichlorodiamine platinum & 41.0 \\
\hline
\end{tabular}

* see text.

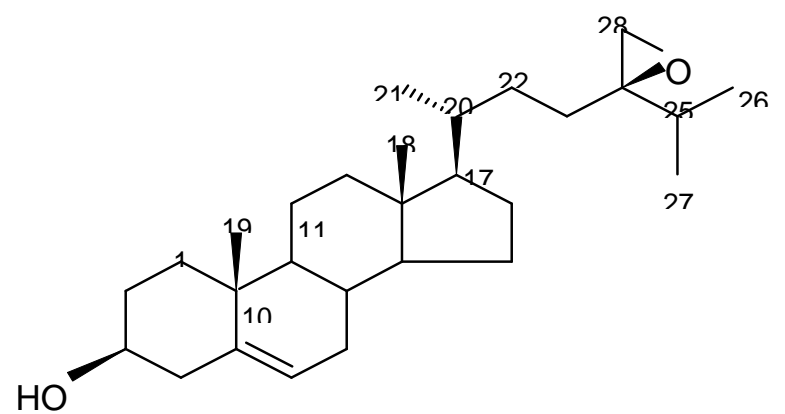

1<smiles>CC(C)C1(CC[C@@H](C)[C@H]2CCC3C4C[C@H](O)[C@]5(O)CC(=O)CC[C@]5(C)C4CC[C@]32C)CO1</smiles><smiles>CC(C)C1(CC[C@@H](C)[C@H]2CCC3C4CC=C5C[C@@H](O)CC[C@]5(C)C4CC[C@]32C)CO1</smiles>

2<smiles>CC(C)[C@@]1(CC[C@@H](C)[C@H]2CCC3C4C[C@H](O)[C@]5(O)CC(=O)CC[C@]5(C)C4CC[C@@]32C)CO1</smiles> 


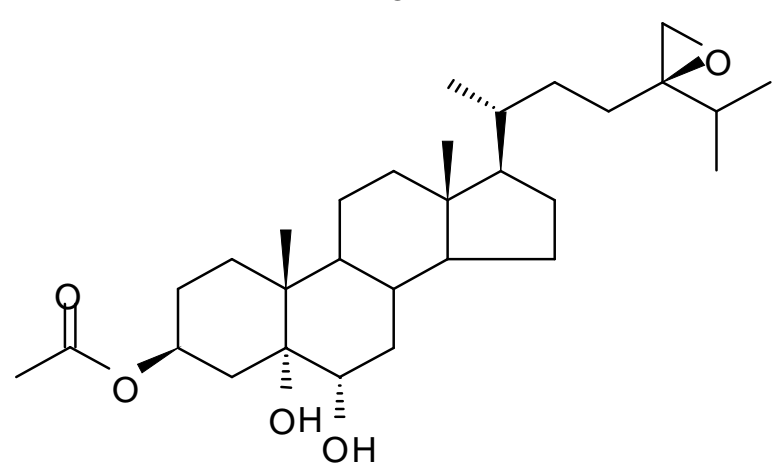

5
4

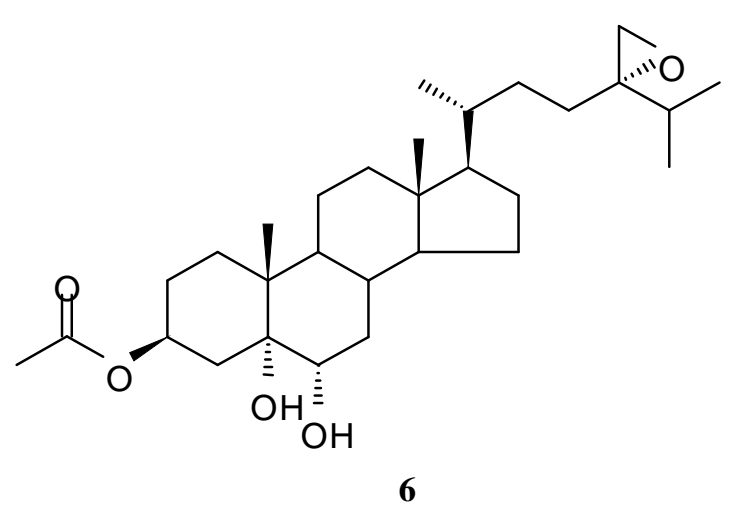

Figure 15. Structures of polyoxygenated 24,28-epoxyergosterols (1, 2 - new natural products, 3-6 - new compounds) from sea anemone Anthopleura midori (reproduced from work 142).

Compounds isolated from natural sources were assessed for the proliferation inhibiting activity in rat glioma C6 and human glioma U251. Compounds 1, 2, 5, 7, and 11 exerted dose-dependent activity toward both glioma cell lines. Compounds 3 and 8 inhibited proliferation of the C6 cells only while 9 and 10 were inactive for both C6 and U251. Epoxyergosterols 1 and 5 were found to be the most active agents regarding $\mathrm{C} 6$ cells with an average IC50 values 2.41 and $10.58 \mu \mathrm{M}$, respectively. Other compounds were less active (Table 29). C6 cells were probably more sensitive then U251 cells. It is interesting that temozolomide exerts its activity towards C6 cells with IC50 approximately $69.58 \mu \mathrm{M}$ (i.e. 29.9. times less active then compound 1 and in 6.6. times les then compound 5) was not active at all against $\mathrm{U} 251$ cells, probably, due to the temozolomide resistance of $\mathrm{U} 251$ cells.

Table 29. Anti-glioma activity of polyoxygenated 24,28-epoxyergosterols from the sea anemone Anthopleura midori [142].

\begin{tabular}{|c|c|c|c|c|c|}
\hline \multirow[t]{2}{*}{ Compound } & \multicolumn{2}{|c|}{$\mathrm{IC}_{50}(\mu \mathrm{M})$} & \multirow[t]{2}{*}{ Compound } & \multicolumn{2}{|c|}{$\mathrm{IC}_{50}(\mu \mathrm{M})$} \\
\hline & $\mathrm{C} 6$ & U251 & & C6 & U251 \\
\hline $1^{*}$ & $2.41 \pm 0.52$ & $40.94 \pm 1.06$ & 7 & $38.38 \pm 3.25$ & $80.45 \pm 4.07$ \\
\hline 2 & $18.59 \pm 0.32$ & $64.26 \pm 3.75$ & 8 & $72.14 \pm 2.70$ & NA \\
\hline 3 & $43.60 \pm 0.93$ & $\mathrm{NA}^{* *}$ & 9 & NA & NA \\
\hline 4 & NA & NA & 10 & NA & NA \\
\hline 5 & $10.58 \pm 0.57$ & $41.91 \pm 2.99$ & 11 & $38.38 \pm 3.25$ & $46.08 \pm 1.65$ \\
\hline 6 & NA & NA & Temozolomide & $69.58 \pm 6.10$ & NA \\
\hline
\end{tabular}

*see text; ${ }^{* *}$ NA: no inhibitory activity at concentration of $100 \mu \mathrm{M}$.

24(R),28-epoxyergost-5-en-3b-ol (1) was applied in $40 \mu \mathrm{M}$ and $80 \mu \mathrm{M}$ concentration in vitro induced apoptosis and necrosis in U251 cells after treatment for 72 hours. In addition, when applied in concentration $80 \mu \mathrm{M}$ it caused $21.83 \%$ increased number of the early apoptotic cells from $0.56 \%$ (control) to $22.39 \%$. Portion of the cells in the G0/G1 cell cycle phase increased by $11.59 \%$ and $9.94 \%$ after $6 \mathrm{~h}$ and $12 \mathrm{~h}$ of treatment with compound $1(80 \mu \mathrm{M})$. The alternation occurring in the cell cycle suggests that epoxyergosterols might arrest U251 cells in G0/G1 phase. Epoxyergosterol $3(40 \mu \mathrm{M})$ also significantly induced apoptosis in $\mathrm{C} 6$ cells increasing the number of early apoptotic cells by $44.66 \%$ and that of late apoptotic cells by $18.41 \%$ [142]. 


\section{Quinones, antraquinones}

Quinones are benzol derivatives with oxo-groups at para- or orto-position possessing some properties of aromatic compounds. Antraquinones are natural compounds based on anthracene structure composed of three benzol rings linked together with a carbonyl group located on the top of each ring. There are medicinal compounds among quinones and antraquinones including bioactives with antiproliferative activity. One novel antraquinone and two already known ones were extracted from actinomycete Streptomyces sp. ZZ406 isolated from the sea anemone Haliplanella lineata. A new anthraquinone was elucidated as 1-hydroxymethyl-8-hydroxy-anthraquinone-3-carboxylic acid (molecular formula C16H10O6). Two antrauinones were previously described as 1,8-dihydroxy-3-methyl-anthraquinone (also known as chrysophanol or chrysophanic acid), and 3,8-dihydroxy-1-methyl-anthraquinone-2-carboxylic acid. It has been found that new anthraquinone had a promising activity against different glioma cells that is comparable with antiglioma effects exerted by doxorubicin (Table 30). At the same time, cytotoxicity of the novel compounds toward normal astrocytes was extremely low with CC50 values greater than $100 \mu \mathrm{M}$ whereas that of doxorubicin is $8.7 \pm 1.2 \mu \mathrm{M}$. Therefore, selectivity index (CC50/IC50) of doxorubicin is 0.9 (for U251 cells), 3.5 (for SHG44) and 4.6 (for U87MG), whereas those values for the novel antraquinone were $->17.5,>12.3$ and $>21.3$, respectively. Presented parameters of antiproliferative activity and selectivity index indicate the presence of substantial advantages of the new antraquinone, at least, in comparison to doxorubicin. Already known antraquinones isolated from Streptomyces sp. ZZ406 also demonstrated high antiglioma efficacy and activity of chrysophanol was significantly greater than the one of doxorubicin [143].

Increased glycolysis rate is known to be necessary for rapid and unlimited cancer cell proliferation and is considered a notable sign of the glioma metabolism. A series of glycolytic enzymes including hexokinase 2 (HK2), 6-phosphofructo-2-kinase/fructose-2 6biphosphatase 3 (PFKFB3), pyruvate kinase M2 (PKM2) and lactate dehydrogenase 5 (LDH5) exert enhanced activity in glioma cells and preferentially in use in cancer cells. New antraquinone was applied in concentration $30.0 \mu \mathrm{M}$ significantly reduced HK2, PFKFB3, PKM2, and LDH5 expression level in the U87MG cells. Based on the results indicating strong activity against glioma cells with extremely high selectivity index and unique antiglioma mechanism the authors of that work assume that the new antraquinone possesses a good potential of antiglioma agent [143].

Table 30. Antiglioma activity of anthraquinones from Streptomyces sp. ZZ406 [143].

\begin{tabular}{lrrrrr}
\hline Compound and structure & & \multicolumn{2}{c}{$\mathrm{IC}_{50}(\mu \mathrm{M})$} & \multicolumn{3}{c}{$\mathrm{C}_{50}(\mu \mathrm{M})$} \\
\cline { 2 - 5 } & & $\mathrm{U} 251$ & $\mathrm{U} 87 \mathrm{MG}$ & SHG44 & Human astrocytes \\
\hline
\end{tabular}

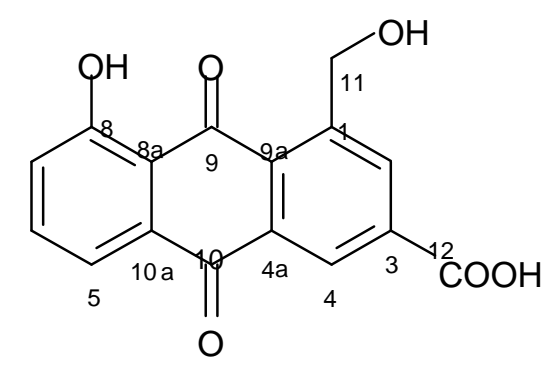

$5.7 \pm 0.3 \quad 4.7 \pm 0.2 \quad 8.1 \pm 0.4 \quad>100$

1-hydroxymethyl-8-hydroxy-anthraquinone-3-carboxylic

acid

$\left(\mathrm{CC}_{50} / \mathrm{IC}_{50}\right)$

$\begin{array}{lll}>17.5 & >21.3 \quad>12.3\end{array}$<smiles>C=C1c2cccc(O)c2C(=O)c2c(O)cc(C)cc21</smiles> 
$\begin{array}{lll}1.2 \pm 0.1 & 0.5 \pm 0.1 \quad 3.0 \pm 0.2 \quad \text { No testing }\end{array}$

Chrysophanol<smiles>Cc1c(C(=O)O)c(O)cc2c1C(=O)c1c(O)cccc1C2=O</smiles>

13.0 $\pm 0.6 \quad 10.4 \pm 0.5 \quad 36.3 \pm 0.2 \quad$ No testing

3,8-dihydroxy-1-methyl-anthraquinone-2-carboxylic acid

\begin{tabular}{lcccc} 
Doxorubicin & $9.6 \pm 1.3$ & $1.9 \pm 0.4$ & $2.5 \pm 1.1$ & $8.7 \pm 1.2$ \\
$\left(\mathrm{CC}_{50} / \mathrm{IC}_{50}\right)$ & $(0.9)$ & $(4.6)$ & $(3.5)$ \\
\hline
\end{tabular}

Marine strain of Streptomyces sp. 182SMLY isolated from a sediment sample collected from the East China Sea was found a source of two new polycyclic antraquinones, which were elucidated as N-acetyl-N-demethylmayamycin (a new analogue of mayamycin, molecular formula $\mathrm{C} 27 \mathrm{H} 25 \mathrm{NO} 8)$ and streptoanthraquinone A (C28H22O8). Cytotoxic and antibacterial agent mayamycin was previously isolated from Streptomyces sp. strain HB202, a symbiotic bacterium of the marine sponge Halichondria panicea, and is considered a first discovered compound in the angucycline class with a C-glycoside at the C-5 of the quinone skeleton [144]. Both anthraquinones remarkably suppressed the proliferation of four different glioma cell lines with IC50 values within a range from 0.5 to $7.3 \mu \mathrm{M}$ (Table 31). Doxorubicin as a positive control exerted its activity with IC50 0.9-9.0 $\mu \mathrm{M}$. IC50 values towards the normal human astrocytes were about $25 \pm 1.3 \mu \mathrm{M}$ for N-acetyl-N-demethylmayamycin and greater than $100 \mu \mathrm{M}$ for streptoanthraquinone A. IC50 ratios between normal astrocytes and glioma cells were 6,4-50 for N-acetyl-N-demethylmayamycin and greater than 14-31 for streptoanthraquinone A. Those values indicate high selectivity index of antraquinones. Also, N-acetyl-N-demethylmayamycin $(0.7 \mu \mathrm{M})$ and streptoanthraquinone $\mathrm{A}(3.3 \mu \mathrm{M})$ significantly induced apoptosis in the glioma U251 cells: the total number of apoptotic cells (early and late apoptotic cells) were increased by $38.76 \%$ and $36.67 \%$, respectively, after $36 \mathrm{~h}$ of treatment when compared to control $(3.58 \%)$ [145].

Table 31. The structure and anti-glioma activities of the polycyclic quinones from marine Streptomyces sp. 182SMLY [145].

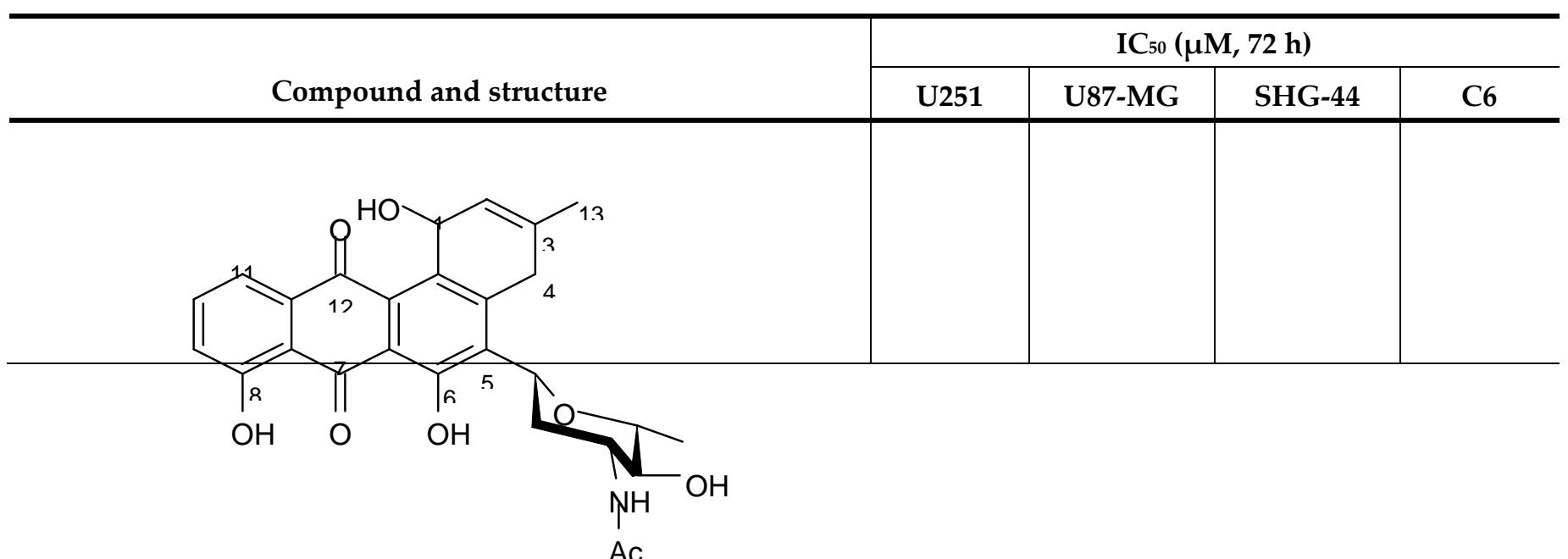




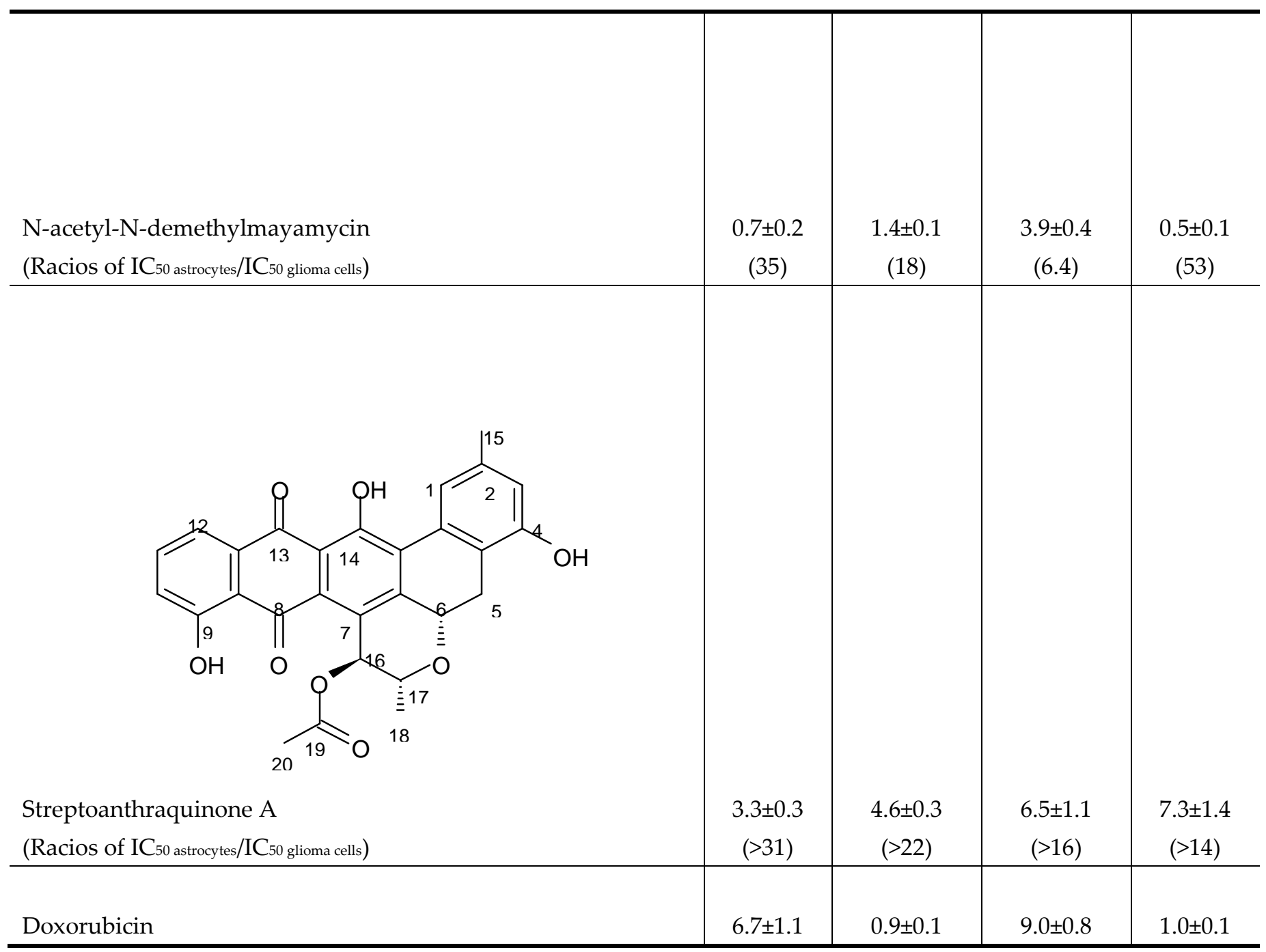

The two anthraquinone derivatives 1 '-deoxyrhodoptilometrin and (S)-(-)-rhodoptilometrin (one of the two stereoisomers of rhodoptilometrin) isolated from the sea lily Colobometra perspinosa (phylum Echinodermata, class Crinoidea) exerted moderate anticancer activity in vitro against human glioblastoma cell line SF-268 [146]. Both compounds from the sea lilies Comanthus sp. also demonstrated toxic effects against C6 glioma cells (Table 32). The higher cytotoxic activity of 1'-deoxyrhodoptilometrin indicates the relevance of the hydroxyl group at C-1' position for anti-glioma activity of the quinone structure. 1'-Deoxyrhodoptilometrin, but the (S)-(-)-rhodoptilometrin, induced a detected apoptotic cell death via caspase $3 / 7$ activity increase: significant higher enzyme activity was found in C6 glioma cells in $24 \mathrm{~h}$ of agitation with concentrations $25 \mu \mathrm{M}$ and higher. 1'-Deoxyrhodoptilometrin applied in concentration $25 \mu \mathrm{M}$ also induced necrotic cell death in glioma cells in $24 \mathrm{~h}$ agitation period that was detected due to increased LDH activity in the supernatant. (S)-(-)-rhodoptilometrin caused a slight increase in the LDH activity only at the highest concentration used $(50 \mu \mathrm{M})$. Both compounds did not cause oxidative stress as no increased accumulation of the reactive oxygen species (up to $50 \mu \mathrm{M}$ ) were noted in the C6 cells. They also did not activate the Nrf2/ARE signaling pathway. In addition, both these compounds did not affect transcription factor NF- $\kappa$ B in the H4IIE cell used as a model system that was stimulated by the cytokine TNF- $\alpha$. Effects of those compounds on the protein kinases involved in different signal transduction pathways associated with cell proliferation (ARK5, Aurora-A, Aurora-B, B-Raf, CDK2, CDK4, COT, EGF-R, ERBB2, FLT3, PDGF-Rb, PLK1, SAK), survival (Akt1, CK2, IGF1-R), angiogenesis (EPHB4, Tie2, VEGF-R2, VEGF-R3), and metastasis (FAK, MET, SRC).were analyzed. The results demonstrated both antraqionones are highly potent inhibitors of various kinases, in 
particular, the ones involved into the cell proliferation and angiogenesis [147]. The protein kinases Aurora-A and Aurora-B were inhibited by both compounds with IC50 values of 3.0 and $1.81 \mu \mathrm{M}$ for 1 '-deoxyrhodoptilometrin and 4.14 and $4.14 \mu \mathrm{M}$ for (S)-(-)-rhodoptilometrin, respectively. Aurora kinases are known as potential anticancer drug targets, since they are involved in the control of chromosome assembly and segregation during mitosis.

Table 32. The structure and cytotoxicity of the anthraquinone derivatives from the sea lilies Comanthus sp. against C6 glioma cells [147].

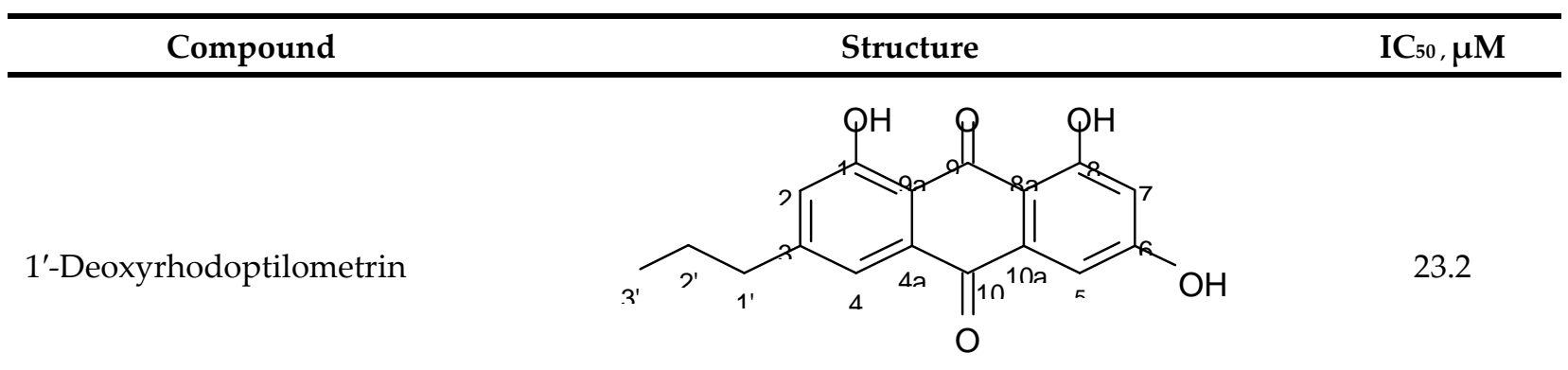

(S)-(-)-rhodoptilometrin

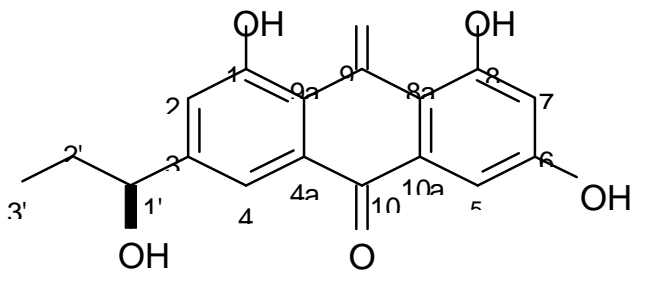

Angiogenesis playing important role in the tumor growth, progression, invasiveness, metastasis includes important signaling pathways such as vascular endothelial growth factor (VEGF) that realizes its activity through the specific VEGF receptors. 1'-Deoxyrhodoptilometrin and (S)-(-)-rhodoptilometrin inhibited the VEGF receptor 2 with IC50 values of 1.87 and $18.76 \mu \mathrm{M}$. That results demonstrates high activity of 1 '-deoxyrhodoptilometrin inhibiting that protein kinase 10 times more effectively than (S)-(-)-rhodoptilometrin. Other important protein kinases that were inhibited by 1'-deoxyrhodoptilometrin and (S)-(-)-rhodoptilometrin were cyclin-dependent kinases (regulation of cell cycle), SAK kinase (mitotic regulator), insulin-like growth factor receptor, focal adhesion kinase (FAK) (cellular adhesion and spreading processes), and epidermal growth factor receptor kinase (cell proliferation).

The epidermal growth factor receptor family of the tyrosine kinases receptors consists of distinct receptors. EGF-R (also known as ERBB-1/HER1) was inhibited by those compounds with IC50 values $4.0 \mu \mathrm{M}$ (1'-deoxyrhodoptilometrin) and $12.4 \mu \mathrm{M}((\mathrm{S})-(-)-$ rhodoptilometrin), ERBB-2 (also known as HER2, neu) was inhibited with IC50 values 6.7 $\mu \mathrm{M}$ (1'-deoxyrhodoptilometrin) and $12.1 \mu \mathrm{M}((\mathrm{S})-(-)$-rhodoptilometrin). ERBB-4 (also known as HER4) was inhibited only by 1 '-deoxyrhodoptilometrin with an IC50 value 9.4 $\mu \mathrm{M}$ [147]. It is already known that a constitutive activation of the mitogen-activated (MAP kinase) signaling pathway occurs in many tumors. It was discovered that 1 '-deoxyrhodoptilometrin substantially decreased ERK MAP kinase (p44/p42) phosphorylation in the glioma C6 cells despite the whole amount of ERK protein remained unchanged. Therefore, 
protein kinase inhibition may be one of the main mechanisms contributing antraquinone anti-glioma activity.

\section{Marine nucleosides: trachycladines}

Naturally occurring modified nucleosides and its synthetic analogues exerting a wide spectrum of biological activity including antibacterial, antiviral, antifungal, and antiparasitic activities, as well as anticancer effects [148] are considered as a key lead compounds for the drug discovery and development. Marine organisms is an inexhaustible source of the modified nucleosides, in particular, sea algae and sponges. Initially marine modified nucleosides trachycladines A and B were isolated from the sponges Trachycladus laevispirulifer [149] and later from other sponge of the genus Theonella. They both contain carbohydrate 2-C-methyl-D-5-deoxyribofuranose part, that was never found in natural sources before, and they differentiate by the nucleic bases (2-chloroadenine for trachycladines A and hypoxanthine for trachycladines B) attached to the carbohydrate part. Trachycladine A represents a carbohydrate-modified analogue of 2-chloro-2'-adenosine, whereas trachycladine B is a carbohydrate-modified analogue of inosine (Figure 16). The first studies revealed a potent in vitro cytotoxic activity of trachycladine $A$ against human colon cancer, leukemia, and breast cancer cell lines with IC50 values ranging from 0.3 to $3.0 \mu \mathrm{M}$ [149]. Trachycladine A exerted significant cytostatic effects on the glioblastoma T98 cell line when applied in concentrations 50 and $100 \mu \mathrm{M}$, and against U87 cell in concentrations 10,50 , and $100 \mu \mathrm{M}$. U87 cell line was found to be more sensitive to trachycladines. $50 \mu \mathrm{M}$ concentration of trachycladine A reduced viability of those cells by $70 \%$, and viability of the T98 cells by 50\% [150].<smiles>C[C@@H]1O[C@@H](n2cnc3c(N)nc(Cl)nc32)[C@](C)(O)[C@@H]1O</smiles>

1<smiles>C[C@@H]1O[C@@H](n2cnc3c(=O)[nH]cnc32)[C@](C)(O)[C@@H]1O</smiles>

2

Figure 16. Structures of trachycladine A (1) and trachycladine B (2).

Two trachycladine analogues, diacetate of the 2,6-dichloropurine derivative (Figure $17,3)$ and N-cyclopropyl trachycladine A (Figure 17,4) exerted greater activity against glioblastoma cells.<smiles>CC(=O)O[C@H]1[C@@H](C)O[C@@H](n2c(C)nc3c(Cl)nc(Cl)nc32)[C@@H]1OC(C)=O</smiles>

3<smiles>[R]Nc1nc(Cl)nc2c1ncn2[C@@H]1O[C@H](C)[C@@H](O)[C@]1(C)O</smiles>

4: $\mathrm{R}=$ cyclopropyl

5: $\mathrm{R}=$ cyclopentyl 
Figure 17. Synthetic analogues of trachycladine A [150].

Comparison of the antiglioma properties of two natural trachycladines showed that trachycladine A definitely is more active compound. Cytotoxicity analysis of several synthetic analogues that nucleosides containing chlorine atom at $\mathrm{C} 2$ of purine ring are generally more active. On the other hand, N-cyclopentyl analogue of trachycladine A (Figure $17,5)$ demonstrated much lower activity than the corresponding $\mathrm{N}$-cyclopropyl analogue and trachycladine A itself. Diacetate structure is following the same C2 chlorination pattern but it lacks the C-6 amino group, it also proved to be highly cytotoxic, probably due to the better cellular uptake because of its higher lipophilic nature. Additional experiments helped reveal the mode of action of active trachycladines relying more on mitotic catastrophes rather than DNA damage. Their activity as autophagic flux blockers was also postulated [150].

\section{Glycosphingolipids and sphingosins}

Glycosphingolipids (GSLs) are characteristic components of all biologic membranes in eucaryotic cells, where they are found in the carbohydrate-rich glycocalyx consisting of GSLs as well as of glycoproteins and glycosaminoglycans. Among the glycolipids, GSLs represent a group of biomolecules containing two basic structural units: hydrophobic ceramide moiety and hydrophilic extracellular oligosaccharide chain protruded from the membrane surface. The ceramide part is made up of a sphingosine moiety composed a long amino alcohol chain with 18-20 carbon atoms and a long fatty acid chain. Sphingosine most frequently found in a glycosphingolipid is a C18-sphingosine, although large amounts of C20-sphingosine have been detected in the brain ganglioside along with C18sphingosine. GSLs are classified into the following subgroups: (i) neutral, which can be subdivided into the cerebrosides containing only one uncharged sugar, diosylceramides with two sugar units, and the neutral glycosphingolipids with more than two (up to 30) uncharged sugars; and (ii) acidic, which can be subdivided into the gangliosides characterized by the presence of one or more neuraminic acid residues, and sulfatides containing at least one sugar residue with a sulfate group. In any case, all glycosphingolipids share a common ceramide lipid unit consisting of a long-chain amino-alcohol fragment presented with either sphingosine or phytosphingosine unit (sphingoid base) linked to a fatty acid via an amide bond [151]. The main types of sugars in GSLs in animals are glucose, galactose, fucose, $\mathrm{N}$-acetylglucosamine, and $\mathrm{N}$-acetylgalactosamine. As a rule, gangliosides contain one or more sialic acids: N-acetylneuraminic acid or N-glycolylneuraminic acid or derivatives there of which are attached to the neutral sugars by an aketosidic linkage. GSLs may be structurally related to one another through the stepwise addition or removal of single monosaccharides. Such glycolipids can therefore be arranged in series. Sialic acid containing glycolipids, i.e. gangliosides occur within all these carbohydrate series. The main families of GSLs start with lactose as the first building block, further extension by $\mathrm{N}$-acetylglucosamine, galactose or $\mathrm{N}$-acetylgalactosamine leads to the formation of oligosaccharides of the lacto-, globo- or the ganglio-series, respectively.

Enhanced interest to GSLs caused by they pivotal roles in numerous physiological processes during cellular development, differentiation, and immune responses, and such phenomena as cell-cell recognition and antigenic specificity. Altered GSL expression can also promote pathophysiological processes like cell invasion, multidrug resistance, neurodegenerative changes, and epithelial-mesenchymal transition in malignant tissue [152]. Moreover, cancer-associated GSLs have been considered as tumor markers, and used as diagnostic markers and targets of cancer treatment [153]. On the other hand, bioactive GSLs exert some pharmacological effects, e.g., antimalarial activity, as well as immunomodulating and antitumor activities [154-155].

Various cerebrosides namely glycosylceramides were isolated from marine sponges (Porifera), ascidians (Chordata), octocorals and sea anemones (Cnidaria), starfishes and sea cucumbers (Echinodermata) [151]. Thus, these marine animals are considered a source of bioactive GSLs. 
A bioactive glycolipid fraction obtained from the lipid extract of starfish Narcissia canariensis was selected for its ability to significantly inhibit KB (human oral epidermoid carcinoma) cells proliferation. The fraction contained three homologous glycosphingolipids with $\beta$-glucopyranoside as a sugar head, 9-methyl-branched 4,8,10-triunsaturated long-chain aminoalcohol as sphingoid base and amide-linked 2-hydroxy fatty acid chains. Their majority (63\%) had an amide-linked 2-hydroxydocosanoic acid chain and was identified as the ophidiacerebroside C (firstly isolated from the starfish Ophidiaster ophidiamus). The minor components differed by the presence one methylene group were corresponding to ophidiacerebroside B and ophidiacerebroside D. It was found that the final glycolipid fraction demonstrated moderate cytotoxic activity on astrocytoma cells obtained after tumor resection of patients with glioblastoma multiforme-primary culture after $24 \mathrm{~h}$ of treatment (Table 33) [154].

Table 33. The cytotoxic activity of glycolipid fractions containing three glucosylceramides from starfish Narcissia canariensis against human astrocytoma cells [154].

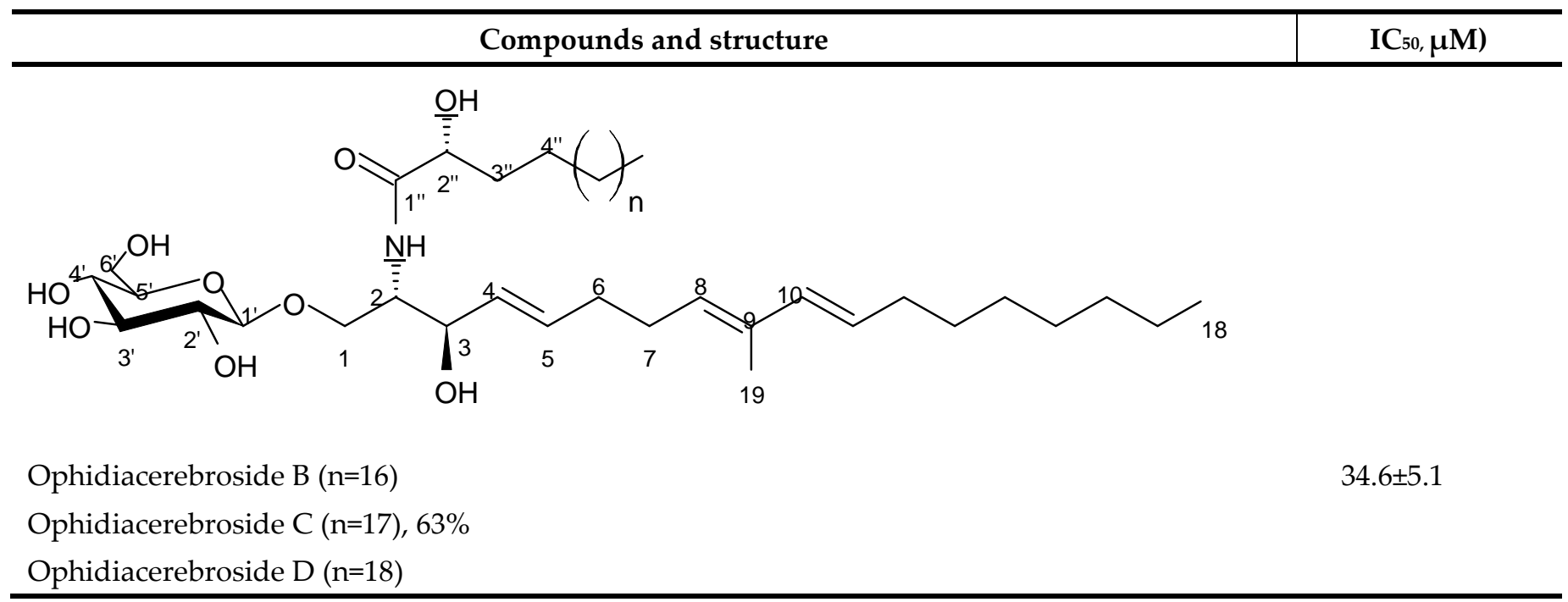

Three glycosphingolipids isolated from the marine sponge Axinyssa djiferi were named axidjiferosides A, B, and C. They contained an unsaturated long-chain amino alcohol as a sphingoid base. The sugar linked to the ceramide was identified as galactopyranose. The sphingoid base was identified as 2-amino-1,3,4-trihydroxy-octadecene. Generally, axidjiferosides were identified as three homologous $\beta$-galactopyranosylceramides composed of 2-amino-(6E)-octadec-6-en-1,3,4-triol and the major one, axidjiferoside A, containing 2-hydroxytetracosanoic acid [155]. These glycosphingolipids in a form of total fraction exerted low cytotoxicity against astrocytoma cells obtained from the tumor resection in patients with glioblastoma multiforme (Table 34).

Table 34. The cytotoxic activity of glycolipid fractions containing three glycosphingolipids from marine sponge Axinyssa djiferi against human astrocytoma cells [155].

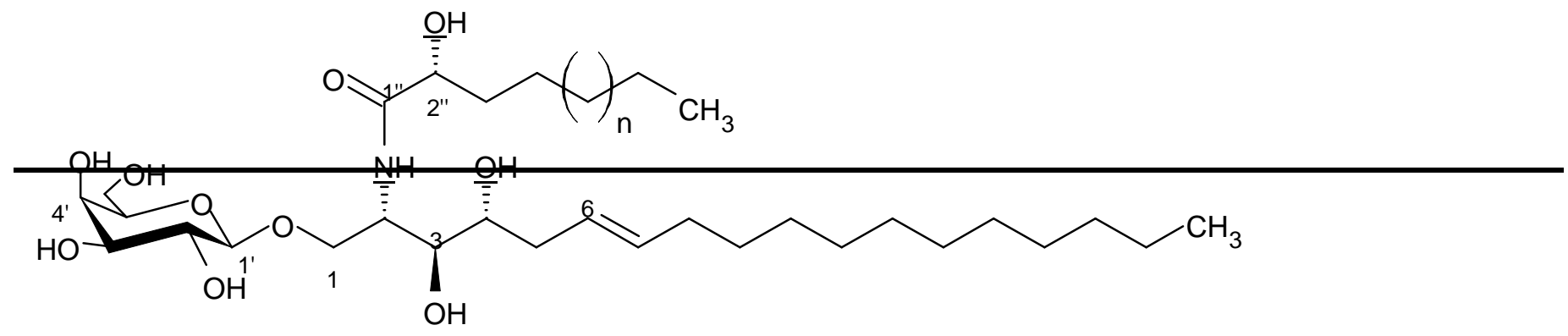


Axidjiferoside A ( $\mathrm{n}=18), 60.8 \%$

Axidjiferoside B $(\mathrm{n}=17)$,

$22.4 \%$

$>60 \mu \mathrm{M}$

Axidjiferoside C ( $\mathrm{n}=19), 16.8 \%$

Sphingosins. Two modified long-chain sphingoid C18 bases: (2R,3R,6R,7Z)-2aminooctadec-7-ene-1,3,6-triol and (2R,3R,6R)-2-aminooctadec-1,3,6-triol, named halisphingosine $\mathrm{A}$ and halisphingosine $\mathrm{B}$, respectively, were isolated from ethyl acetate fraction of methanol extract of the marine sponge Haliclona tubifera. Ethyl acetate fraction in contrast to the aqueous and hexane fractions exerted cytotoxic effect in the U87 human glioma and SH-SY5Y human neuroblastoma cell lines (Table 35) [156].

Table 35. In vitro growth inhibitory activity of ethyl acetate fraction containing two sphingosines from marine sponge Haliclona tubifera against human glioma U87 and human neuroblastoma SH-SY5Y cell lines [156].

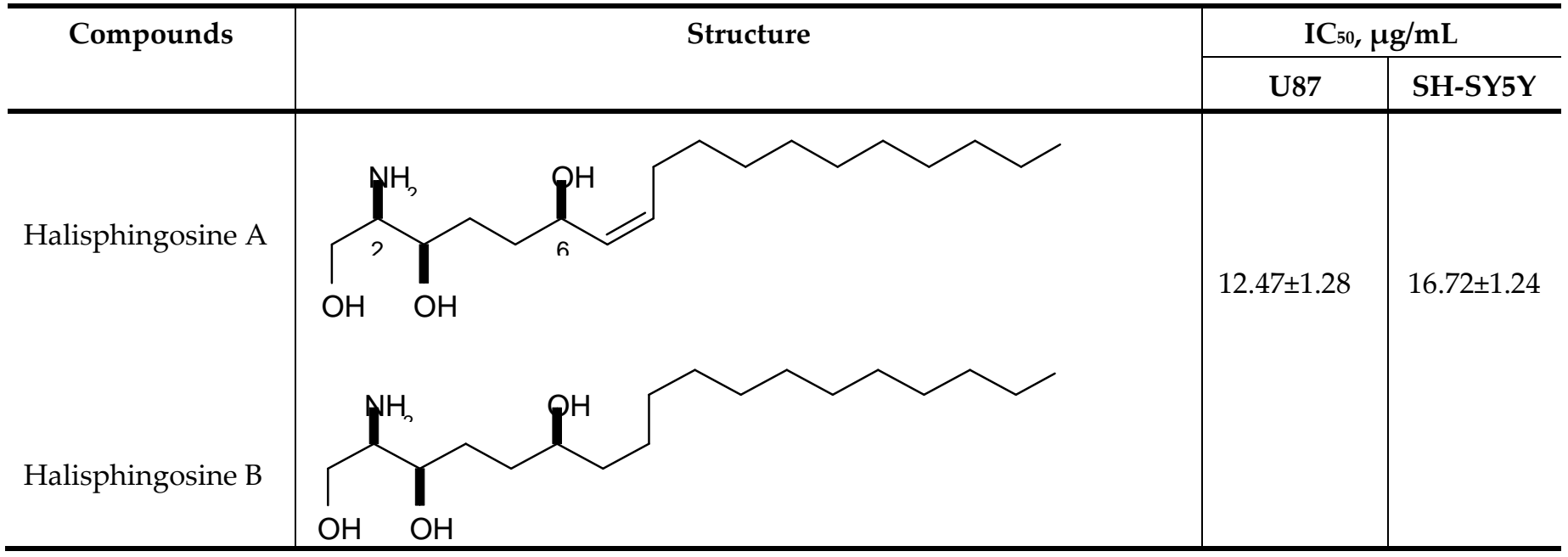

Within the recent years, sphingosines are gaining recognition as important signaling mediator of apoptosis. Possible mechanisms involved in sphingosine-mediated cancer cell death are related to its kinase modulating potential, mainly, regarding protein kinase $\mathrm{C}$

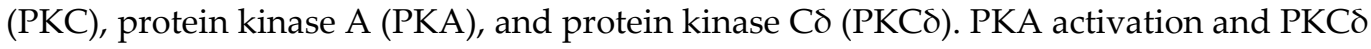
cleaving are associated with regulation of the dimeric 14-3-3 protein function displaying a vital clue of the control pro-apoptotic mediators such as BAD and signal-regulating kinase 1 (ASK-1). Activation of the Jun N-terminal kinases (JNK) is subsequently regulated by ASK-1 [157].

Second suggested mechanism of action may be related to the sphingolipid biosynthetic pathway, which is known to be activated in response factors resulting in accumulation of ceramide and sphingosine in apoptotic cells [157]. Halisphingosines A and B may be a substrate for ceramide synthase and sphingosine kinase resulting in sphingosine converting either into ceramide or sphingosine-1-phosphate (S1P). Ceramide exert antiproliferative effects inducing apoptotic mediators, whereas S1P promotes cell survival and inhibition of apoptosis [158]. Based on the experimental results as well as on knowledge about sphingolipid synthesis and signaling role in debilitation of cell proliferation in U87MG glioma cells [159] the authors came to a conclusion that possible cytotoxic 
mechanism of the sphingosines from $\mathrm{H}$. Tubifera marine sponge is elucidated by induction of apoptotic mediators and ceramide production. In addition, ethyl acetate fraction was shown to be able to inhibit the production of peroxyl radicals [156].

\section{Psammaplins}

Natural compound named psammaplin A possessing a wide spectrum of biological activities was initially isolated by several independent research teams from marine sponge Psammaplysilla (revised to Pseudoceratina) sp. or from unidentified sponges back in 1987 [160]. Actually, psammaplin A is found in marine microalgae, cyanobacteria, and in the heterotrophic bacteria living in association with invertebrates (e.g., sponges, tunicates, and soft corals). Psammaplin A was found to have unique symmetrical structure of the disulfide bromine tyrosine dimers with phenol properties and became a starting substance for the whole psammaplin family. Later, psammaplin A was synthesized by Hoshino et al. [161] that allowed to produce a wide variety of its derivatives and then investigate their antitumor and other activities [162].

Psammaplin A consists of a symmetrical disulfide with a cystamine linker functionalized on both sides by tyrosine-derived $\alpha$-(hydroxyimino)acyl moieties [163]. Complete formula of psammaplin A is bisprasin, N,N"-(dithiodi-2,1-ethanediyl) bis [3-bromo-4-hydroxy- $\alpha$-(hydroxyimino)-benzenepropanamide. Generally, it is supposed to occur in natural sources in the form of monomers or dimers. The symmetrical disulfide dimer psammaplin A belongs to the open-chain $\alpha$-oximinoamides and represents the first isolated natural product containing oxime and disulfide moieties from marine sponges. Later, psammaplins C, E, F, G, and K were also obtained [163].

Psammaplin A was confirmed to possess a wide spectrum of pharmacological effects including antiviral, insecticidal, active chemical defense, and eryptosis-induction activities as well as antiproliferative effects against various cancer cell lines including triplenegative breast (MDA-MB-231), doxorubicin-resistant human breast (MCF-7/adr), colon (HCT15), ovarian (SK-OV-3), lung (A549, LM4175), bone (BoM1833), endometria, brain (BrM-2a), skin (SK-MEL-2), and central nervous system (XF498) cancer cell lines [162,164]. Cytotoxic effects of psammaplin A are related to the multiple enzyme inhibition such as topoisomerase II, farnesyl protein transferase, mycothiol-S-conjugate amidase, leucine aminopeptidase, DNA polymerase $\alpha$-primase, aminopeptidase $N$. In addition, psammaplin A activates peroxisome proliferator-activated receptor gamma (PPAR $\gamma$ ) and induces apoptosis in MCF-7 cells [165]. Psammaplin A and its derivatives, psammaplin F and psammaplin $\mathrm{G}$ were discovered as highly potent inhibitors of DNA methyltransferase (DNMT) and histone deacetylases (HDAC) playing critical roles in the epigenetic regulation of gene expression [163]. Structural modification with the following investigations of structure-activity relationships demonstrate that disulfide bonds and the oxime moieties are indispensable for the antibacterial and antiproliferative activities of psammaplin A.

Psammaplin A (Sigma Chemical Co.) in the in vitro experimental setting suppressed cell viability in the glioblastoma U373MG with IC50 values $5 \mu \mathrm{g} / \mathrm{mL}$ after $18 \mathrm{~h}$ agitation period (Table 36) [166].

Table 36. The structure and anti-glioma activity of psammaplin A against glioblastoma U373MG cells [166].

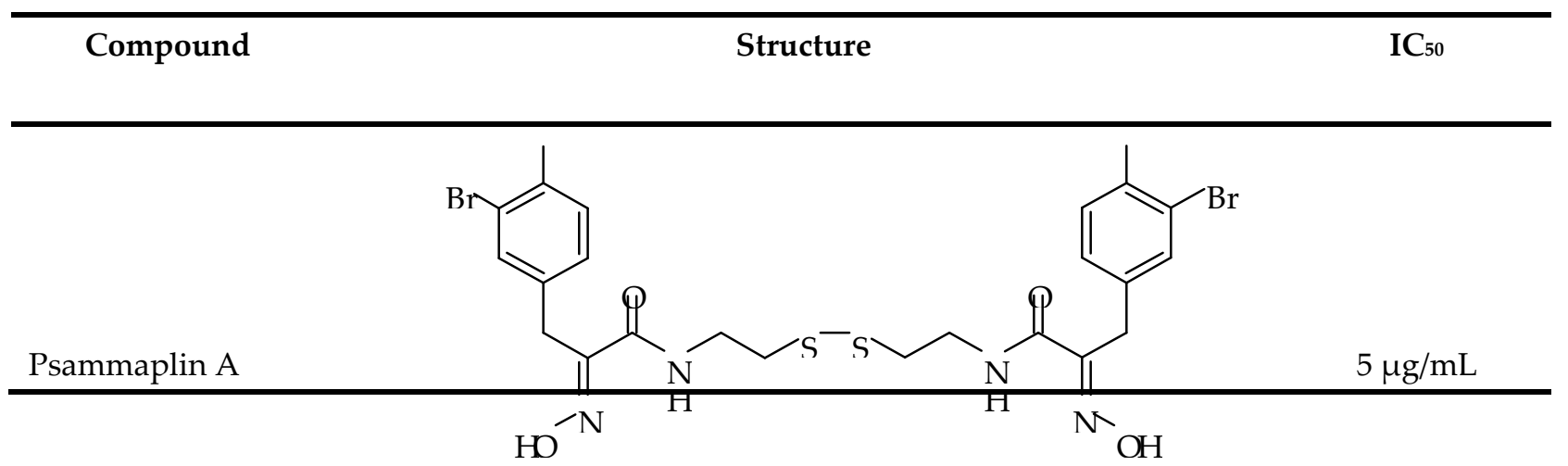


One of the important effects of psammaplin A with high clinical value is related to its influence on the radiosensitivity of the human cancer cells. Investigation of the psammaplin A influence on the radiosensitivity of the glioblastoma U373MG cells have shown that pretreatment with psammaplin A resulted in increased radiosensitization U373MG cells and psammaplin A significantly enhanced radiation induced cell death in U373MG. The dose enhancement ratio (DER) was defined using the experimental dose necessary for the survival fraction obtaining 0.5 as a reference. DER for psammaplin $A$ in U373MG cells was 1.29. It is known that DNMT1, DNMT3A, and DNMT3B are the main functional methyl transferases responsible for setting and maintaining DNA methylation in mammas. Psammaplin A was shown to provoke dramatic reduction of the DNMT1 and DNMT3A expression in the U373MG cells and does not affect DNMT3B expression (at least in a dose $5 \mu \mathrm{g} / \mathrm{mL}$ ) [166].

Previously apoptosis was considered as a potential radiosensitization mechanism. Different results were reported indicating the role of apoptosis as a radiosensitizing mechanism induced by DNMT inhibitors. Some studies have shown that combination of radiation and DNMT inhibitor (zebularine) does not increase significantly sub-G1 population of apoptotic cells [167]. On the other hand, DNMT inhibitor (5-aza-2'-deoxycytidine) was demonstrated to induce radiosensitization in the gastric cancer cell line via induction of accelerated rate of apoptosis that was indicated by increased expression of the p53, RASSF1 and DAPK gene expression [168]. Psammaplin A was reported earlier to exert cytotoxic influence on the cancer cells via selective induction of the genes related to apoptosis [168]. This effect of psammaplin A was assumed to lead to the increased radiation induced apoptosis. However, such radiation induced apoptosis was noted in the A549 cell line only and was not found in the U373MG line. That may be explained, at least partially, by the state of the p53 expression. Cell line U373MG contains mutated p53 may be relatively more resistant ot the radiation apoptosis because generally apoptosis is linked to p53 protein. DNA recover (repair) is another process taking part in the cellular radiosensitizing determination, and DNA reparation activation in the cancer cell after sublethal DNA damage induced by radiation may be on the resistance factors. Gamma-H2AX been identified as a marker of DNA double-strand break and immune cytochemical analysis with anti- $\gamma \mathrm{H} 2 \mathrm{AX}$-antibodies showed that $\gamma \mathrm{H} 2 \mathrm{AX}$ expression zones in cells treated with radiation only goes down in time but can be prolonged (remains unchanged) within 24 hours period in the U373MG cells treated with DNMT inhibitor before radiation procedure [166]. These results suggest that inhibition of the DNA damage recovery process is a mechanism that makes a base for radiosesitisizing effects of the DNMT inhibitors in glioma cells. Therefore, psammaplin A possesses a potential to increase radiosensitivity in U373MG glioblastoma cells probably via suppression of the DNA reparative processes.

One of the anti-glioblastoma mechanisms of psammaplins may be related to the induction of the autophagic flux in tumor cells. This phenomenon is of great importance because at present targeting autophagic pathways is thought might play a critical role in designing novel chemotherapeutic approaches in the treatment of human cancers, and the prevention of tumor-derived drug resistance. A number of pharmacological compounds including psammaplin A shown to induce autophagy in various human tumor cells, and some of these compounds were shown to affect the expression of TP53 protein in cancer cells via cell cycle arrest in the G0/G1 phase and expression of cyclins [169].

Psammaplin A isolated from the Psammaplinaplysilla sponge decreased cell viability of glioblastoma U87MG cells in a dose-dependent and time-dependent manner with IC50

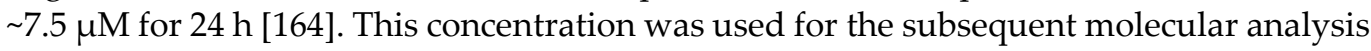
of expression of the tumor protein (TP)-p53 family members and their autophagic target genes. Psammaplin A led to a marked increase $(4.3 \mathrm{x}$ fold $)$ in the protein levels for TP73 $\alpha$ and dramatically induced TP73 $\alpha$ phosphorylation $(8.7 \mathrm{x}$ fold $)$ that may be a mechanism 
reducing tumor cell survival and inducing cell death. The present data demonstrate that the autophagy pathway is one of the molecular mechanisms leading to the tumor cell survival modulation [170]. Although autophagy can serve as a pro-survival phenomenon, it often delays tumor cell death via apoptosis, essentially contributing to the demise of tumor cells upon treatment with the anticancer compounds of various origins. TP53 family proteins were found to play a critical role in autophagy signaling [171] and induce the multiple molecular pathways including transcriptional activation of genes targeting autophagic machinery [172]. It was found out that psammaplin A activates transcription of autophagic genes through TP53 family member's transcriptional function. It upregulates expression levels of ATG5 and UVRAG in U87MG cells and promotes ATG5 and UVRAG promoter activities. Psammaplin A have been demonstrated also to be able to stimulate expression of autophagic proteins involved into autophagy signaling in human glioblastoma tumor cells in vitro. Treatment of U87MG cells with $7.5 \mu \mathrm{M}$ psammaplin A led to a substantial increase in the ATG5 and UVRAG protein expression [164]. Therefore, psammaplin A has a capacity to upregulate expression of autophagic signaling intermediates in human glioblastoma cells in vitro through a transcriptional regulation by TP53 family members.

Low efficiency of the chemotherapy of the brain malignancies due to the high chemoresistance of the glioblastoma stem cells remains problematic for modern neurooncopharmacology. This particular subpopulation of the tumor cells governs tumor initiation and recurrence, they are particularly difficult to be eradicated with chemotherapy. One of the causes of that phenomenon is an elevated expression of P-glycoprotein (Pgp), which is an efflux pump that recognizes chemotherapeutics including temozolomide and other antiglioma drugs as substrates and pushes them out of the cells [173,174]. Implementation of the direct Pgp inhibitors in combinatorial therapy was rather not successful because of serious adverse events and toxicity. This toxicity is attributed to inhibition of Pgp present in healthy tissues and unexpected drug-drug interactions. Indirect inhibition of Pgp via influencing the carbonic anhydrase activity was thought an alternative approach. Tumor acidosis is known as a hallmark of cancer. Membrane-bound carbonic anhydrases IX (CAIX) and/or XII (CAXII) colocalized with the membrane drug efflux protein, Pgp, in a range of drug resistant cancer cells including glioblastoma, maintain the intracellular/extracellular $\mathrm{pH}$ for efficient Pgp activity, and optimal tumor growth, invasion, and metastasis [175]. Nowadays, CAIX- and CAXII-specific inhibitors are considered as potential antitumor agents that indirectly reduce Pgp activity and resensitize solid tumors to Pgp substrates [176].

One of the most potent inhibitors of CAIX and CAXII enzymes today is one of the psammaplin A derivatives, namely, psammaplin C isolated from the marine sponge Pseudoceratina purpurea in 1991 [177]. The structure of psammaplin C comprises several structural elements: (I) a 3-bromo-4-hydroxy benzylidene moiety, (II) an oxime group, and (III) an aminoethane sulfonamide chain (Figure 18). Alteration of the carbon anhydrase inhibiting activity of psammaplins on the human Ca isoenzyme panel showed that psammaplin C had exceptionally strong inhibition activity for hCA XII, with an inhibition constant (Ki) $0.79 \mathrm{nM}$. For comparison: acetazolamide, the par excellence therapeutically established CA inhibitor, had shown inhibition actovoty for hCA XII, with a Ki $5.7 \mathrm{nM}$ [178]. Subnanomolar CA inhibition is uncommon. Moreover, one of the synthesized psammaplin $C$ derivatives, which is a free oxime derived from the thiadiazoyl sulfonamide scaffold, showed very strong inhibition of all the CA isozymes, in particular CA XII with a Ki of $0.56 \mathrm{nM}[179]$. 


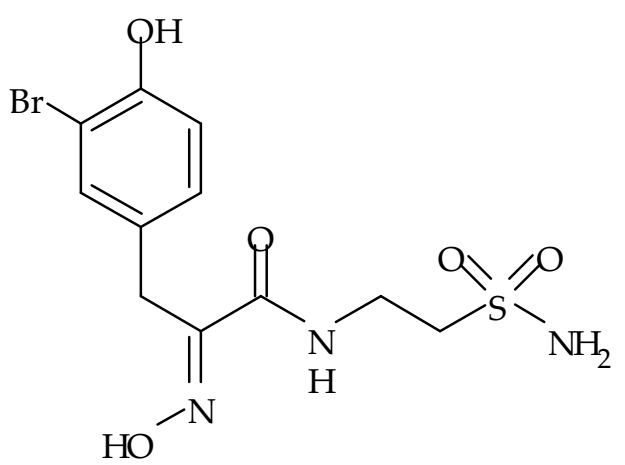

Figure 18. The chemical structure of psammaplin C

Experimental studies with mice with orthotopically implanted glioblastoma neurospheres derived tumors (with coexpression of Pgp and CAXII) or xenografts derived from the patients showed that co-therapy of temozolomide with a CA XII inhibitors may more effectively affect glioblastoma by suppressing an important temozolomide resistance mechanism. CA XII inhibitors themselves did not affect tumor growth in the mouse model studies and did not prolong life span of experimental animals, but significantly enhanced efficacy of temozolomide. Simultaneously, application of the CA XII inhibitors was particularly effective against the glioblastoma with high anti-TMZ resistance. The combination of TMZ with CA XII inhibitors rescued the antiproliferative and proapoptotic effects of TMZ, as verified by reduced intratumor-positive immunostaining for Ki67 and increased activation of caspase-3 [175,179]. Thus, although TMZ alone reduced Pgp expression and activity in neurospheres, this reduction was not sufficient to yield antitumor efficacy in vivo. The greater inhibition of Pgp, as achieved by combining the potent CA XII inhibitor (psammaplin C derivative) with $\mathrm{TMZ}$, was however able to restore the intracellular cytotoxic levels of TMZ and facilitate enhanced drug efficacy. It should be emphasized that psammaplin $C$ shows no toxicity and is only effective if used in combination with a chemotherapy [175].

Therefore, a new combination therapy, based on a CA XII inhibitor with TMZmay be considered as a potentially viable clinical tool to overcome Pgp-mediated TMZ-resistance in glioblastoma stem cells as well as more effective therapy of the brain tumors [179].

\section{Xyloketals}

The xyloketal group of natural compounds was isolated from the mangrove fungus Xylaria sp. This group of related ketals is structurally unique [180]. Xyloketal A has C3 symmetry with a cis-junction between the tetrahydropyran and tetrahydrofuran rings (Figure 19), the other members are missing axial symmetry. The methyl groups at C-5 of $\mathrm{C}$ rings are also oriented syn to other methyl groups at C-2 placed between the oxygens in the spiroketal functions. The angular skeleton of xyloketal B, a bis adduct analogue of the tris adduct xyloketal A, is more stable than the linearly condensed xyloketal C, which spontaneously rearranges in solution to the more stable angular structure of xyloketal B. Xyloketal D is an acetylated mono adduct structure, and xyloketal $\mathrm{E}$ is a tetrahydrofuranlinked angular bis adduct related to xyloketal B. Some studies reported xyloketal B exerts several bioactive functions including anti-stress and anti-ageing, neuroprotective effects, and antioxidant activity [181].<smiles>C[C@H](O)O/C=C1/CO[C@@]2(C)OC[C@H](C)[C@H]2C1</smiles> 
Figure 19. The structure of xyloketal A from the mangrove fungus Xylaria sp. [180]

24 hours long treatment with xyloketal B in various concentrations of (from 31.25 to $1000 \mu \mathrm{M}$ ) reduced U251 cell viability in a concentration-dependent manner. Cell viability was significantly decreased by $85.4 \% \pm 2.9 \%, 61.4 \% \pm 4.3 \%, 12.2 \% \pm 2.6 \%$, and $1.3 \% \pm 0.1 \%$ in comparison to control for $125 \mu \mathrm{M}, 250 \mu \mathrm{M}, 500 \mu \mathrm{M}$, and $1000 \mu \mathrm{M}$ xyloketal B concentration, respectively $(\mathrm{p}<0.05)$. Nonlinear curve fit was carried out to evaluate the doseresponse of xyloketal B with IC50 287.1 $1.0 \mu \mathrm{M}$ (Table 37). Xyloketal B applied in the concentration range $37,5-300 \mu \mathrm{M}$ exert sell proliferation inhibiting activity depending on the period of application and concentration. It should be emphasized that xyloketal B in concentrations $<300 \mu \mathrm{M}$ generally exerted only cell proliferation inhibition activity but not a cytotoxic effect. In concentration greater than $300 \mu \mathrm{M}$ xyloketal B significantly inhibited cell migration in U251 line [182].

Table 37. Chemical structure and antiglioblastoma U251 activity of the xyloketal B from mangrove fungus Xylaria sp. (No. 2508) [182].

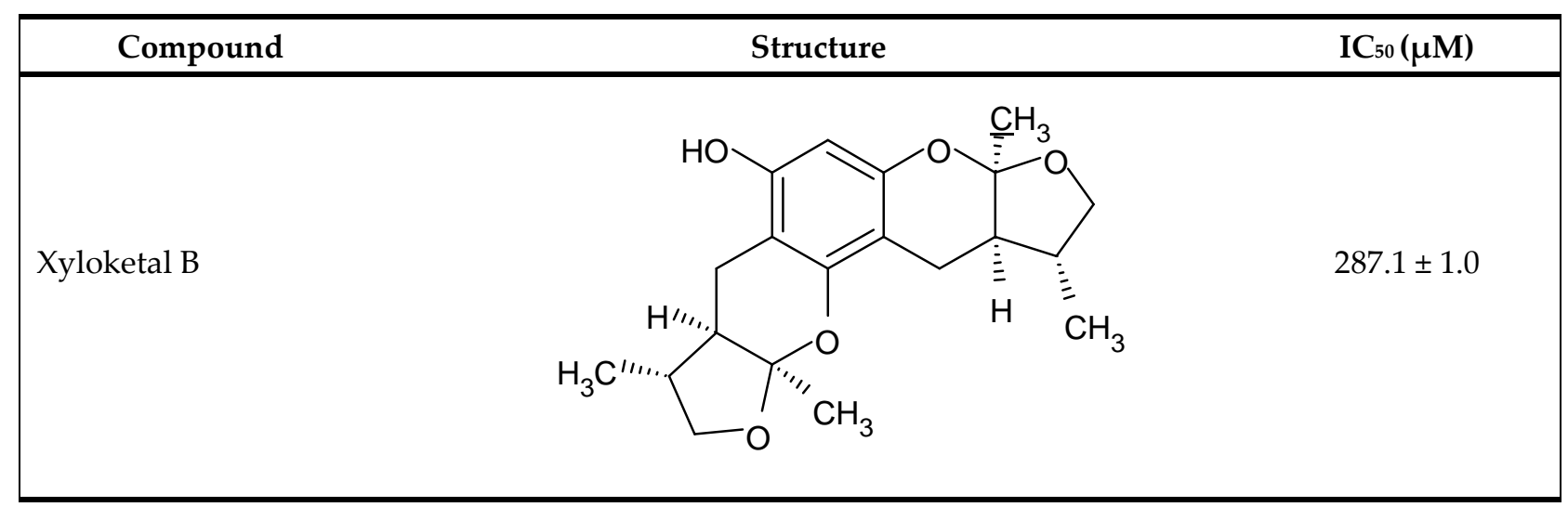

Experiment devoted to investigation of the mechanisms providing antiproliferative activity xyloketal $B$ have shown that p-Akt and p-ERK1/2 protein expression in the U251 cells was significantly decreased after xyloketal B treatment in concentration $300 \mu \mathrm{M}$ for 24 hours. Therefore, reduced cell viability, proliferation, and cell migration in glioblastoma is caused by suppression of the signaling pathways PI3K/Akt and MEK/ERK as well as by the TRPM7 current blockade without alteration of the TRPM7 protein expression in glioma cells. Authors suppose that the marine compound xyloketal B may be a promising drug candidate for anti-glioblastoma therapy [182].

\section{Pheophorbide $a$}

Pheophorbide a is a typical representative of the organic class compounds tetrapyrroles, which as suggested by the name, consists of four pyrrole-derived 
substances linked either in linear or cyclic fashion via methine bridges. There are well known and studies members in this class of molecules such as hemes giving blood its red color and the chlorophylls, which are responsible for the green color of plants, algae, and some bacteria, as well as cobalamin, siroheme, coenzyme F430, heme d1, and the photopigments bilins [183]. Pheophorbide a is a product of the chlorophyll breakdown containing porphyrin ring, which has been reported to be an essential link for the photoactivation assays and photodynamic therapy $[5,7,32]$. Molecular formula of pheophorbide a is $\mathrm{C} 35 \mathrm{H} 36 \mathrm{~N} 4 \mathrm{O} 5$ and its IUPAC name is (3S,4S)-9-ethenyl-14-ethyl-21-(methoxycarbonyl)4,8,13,18-tetramethyl-20-oxo-3-phorbinepropanic acid. Pheophorbide a purified from an edible red seaweed Grateloupia elliptica was shown to exhibit strong anticancer activity with no direct photo-irradiation against various cancer cell lines including mouse melanoma cells (B16-BL6), human epithelial carcinoma cells (HeLa), human cervical cancer cells (SiHa), and human ovarian cancer cells (SK-OV-3) with IC50 values $18.3 \pm 2.9,9.5 \pm$ 2.3, $13.2 \pm 2.6$, and $7.0 \pm 2.0 \mu \mathrm{g} / \mathrm{mL}$, respectively. However, pheophorbide a exerted the strongest anticancer effect against U87MG glioblastoma cells that was comparable with activity of the positive control drug paclitaxel [184]. Cytotoxic effect of pheophorbide a was not observed in the normal human umbilical vein endothelial cells (HUVEC whereas the positive control paclitaxel was found to demonstrate significant and notable cytotoxic activity (Table 38).

Table 38. Anti-glioma activity pheophorbide $a$ from a red seaweed Grateloupia elliptica in U87MGcell line, in vitro [184].

\section{Compound} Structure

$\mathrm{IC}_{50}(\mu \mathrm{g} / \mathrm{mL})$

U87MG HUVEC

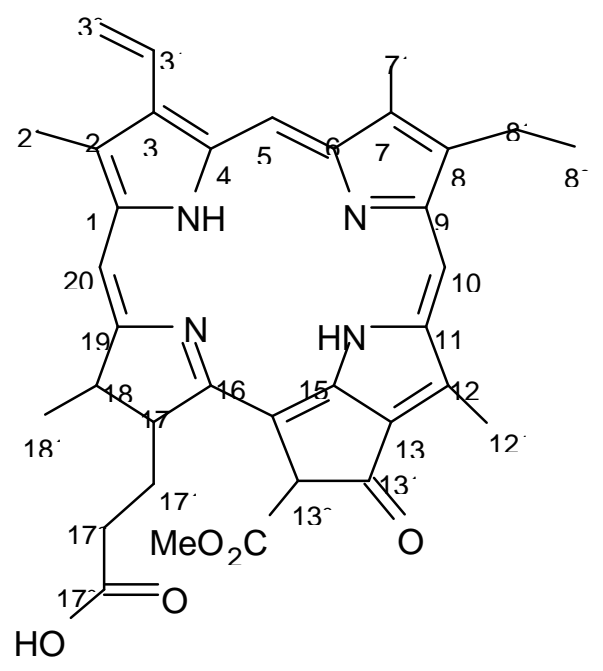

Pheophorbide $a$

$2.8 \pm 1.0$ $>50$

Paclitaxel

$3.2 \pm 1.2$

$0.3 \pm 1.0$

(positive control)

The glioblastoma growth inhibition activity exerted by pheophorbide a was related to the cell cycle arrest in the G0/G1 phase and apoptosis as well as with genomic DNA degradation. Pheophorbide a specifically inhibited only growing U87MG cells but did not affect resting ones. The results obtained through the studies suggest that pheophorbide a 
isolated from G. elliptica could be a good source of glioblastoma-specific therapeutics with no clinically notable side effects [184].

\section{Phorboxazoles}

In 1995 two unique compounds named phorboxazole A and its C13 epimer phorboxazole B were isolated from the marine sponge Phorbas sp. Their complex chemical structure had given a base to consider these phorboxazoles as a new class of natural products containing an unprecedented array of oxane, oxazole, macrolide, and polyene moieties. In addition to the pronounced antifungal activity against Candida albicans shown previously in the in vitro experiments, phorboxazoles were reported to provide extremely high cytostatic effects towards the National Cancer Institute's panel of 60 tumor cell lines with the mean GI50 values less than $1.6 \times 10^{-} 9 \mathrm{M}$ [185]. Later, a complete synthesis of phorboxazole A was performed [186] that gave a start to a series of studies devoted to fabrication of the synthetic phorboxazole derivatives. Thus, phorboxazoles were suggested to be the ones of the most potent natural cytostatic agents that are discovered yet [187].

Experimental studies comparing antiproliferative activity of the phorboxazole derivatives against human glioblastoma cell line U373 have shown these tumor cells in a concentration-dependent fashion with low nanomolar IC50 values were inhibited by synthetic phorboxazole A as well as its analogues 45,46-dehydrobromo-phorboxazole A bearing an alkyne part in C45-C46 position of the terminal bromide, and 33-O-methyl-phorboxazole A containing mixed methyl ketal instead of the C33 positioned hemiketal (Table 39). IC50 values for other synthetic phorboxazole analogues, especially, for 32-methylphorboxolide A (C1-C32 analog), 31-methyl-phorboxylate (C31-C46 analog), 29-phorboxamide A (hydrated analog), C1-C38 of phorboxazole A, and 18-methyl-phorboxylate (C18-C46) were experimentally shown to be greater than $2 \mathrm{mM}$.

Table 39. Structure and antiproliferative activity of synthetic phorboxazole A and its analogues against human glioblastoma cell line U373 [187].

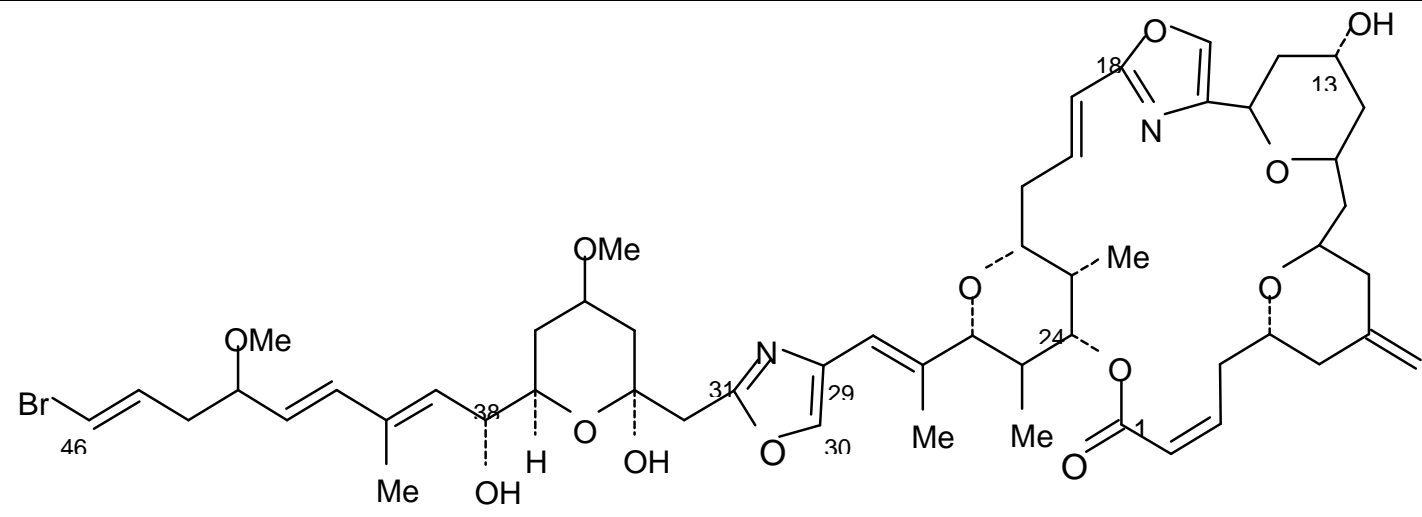

Phorboxazole A

phorboxazole A 


\begin{tabular}{lc}
\hline 31-Methyl-phorboxylate & $>2000$ \\
\hline 29-Phorboxamide A & $>2000$ \\
\hline C1-C38 of phorboxazole A & $>2000$ \\
\hline 18-Methyl-phorboxylate & $>2000$ \\
\hline
\end{tabular}

Structure-activity relationship studies demonstrated that just simple modifications like replacement of the terminal vinyl bromide of phorboxazole A with an alkyne, or C33 hemiketal with a mixed methyl ketal did not lead to substantial loss of anticancer activity. Neither C1-C32 macrolide-containing domain, nor C31-C46 side-chain portion were separately sufficient to sustain the potent anticancer activity of phorboxazole A. The simple covalent joining of the macrolide and side-chain parts of phorboxazole A via an amide at C29-C31 instead of the planar vinyl-substituted oxazole at this position of phorboxazole A was not sufficient to regain appreciable activity. Maintaining the central oxazole, but truncating the side chain by omission of the lipophilic C39-C46 polyene domain resulted in abolished antitumor activity. Deletion of the $\mathrm{C} 2-\mathrm{C} 17$ portion of the phorboxazoles containing oxazole, acrylate, and bispyran moieties similarly resulted in activity loss. These findings suggest that macrolide, central oxazole, and polyene side chain portions of phorboxazoles are necessary for a potent anticancer activity [187].

\section{Phlorotannins}

Phlorotannins are the tannin derivatives existing in a form of polyphenols that built as a result of the phloroglucinol unit polymerization. Phloroglucinol (1,3,5-trihydroxybenzene) is a phenolic compound with an aromatic phenyl ring and three hydroxyl groups. According to linkages between phloroglucinol units and the number of hydroxyl groups, Phlorotannins can be classified into six subgroups in accordance to the linkages between phloroglucinol units and the number of hydroxyl groups namely phlorethols, fuhalols, fucols, fucophlorethols, eckols and carmalols. Phlorotannins containing both phenyl and phenoxy units and having different molecular weights are mainly present in the marine brown algae. The most widely known phlorotannins are phloroglucinol, eckol, dieckol, 8,8'-bieckol, 6,6'-bieckol, dioxinodehydroeckol, phlorofucofuroeckol and a few others [188].

Eckol is a precursor compound illustrating the dibenzo-1,4-dioxin class of phlorotannins and containing phloroglucinol components linked to each other in multiple fashions (Figure 20). Eckol is known to be produced in several marine organisms, in particular, in brown algae including Ecklonia cava (Laminariaceae), and red algae. Numerous studies have shown that eckol exerts antioxidant, anti-diabetic, anti-hyperlipidemic, anti-inflammatory, hepatoprotective, neuroprotective, radioprotective, anti-obesity, anticoagulative, anti-hypertensive, anti-photo aging, anti-histaminic, antibacterial and antiviral activities, as well as anticancer and cytotoxic activity [189].<smiles>Oc1cc(O)cc(Oc2c(O)cc(O)c3c2Oc2c(O)cc(O)cc2O3)c1</smiles> 
Figure 20. The structure of eckol

Hyun et al. [190] investigated the effect of eckol on stem cells and malignancies in glioma stem-like cells. The study was performed with the use of glioma cancer cell lines U87MG and U373MG. Patient-derived glioma stem-like cells X01GB and X03AOA were established from acutely resected human tumor tissues. The X01GB line was derived from a patient with a glioblastoma multiforme, and X03AOA was derived from a patient with anaplastic oligoastrocytoma.

It was shown before that glioma subpopulation expressing CD133 protein is enriched in cancer stem-like cells showing greater tumorigenic potential than CD133 negative cells. In a similar way to neural stem/progenitor culture, those glioma stem-like cells expressing CD133 can be enriched in a serum-free medium supplemented with growth factors, where glioma cell fraction continue to proliferate and form spheres instead of a monolayer [191]. Treatment of the sphere-cultured glioma cells with eckol in concentrations from 50 to 90 $\mu \mathrm{M}$ also decreased the expression of the glioma stem-like cell markers CD133, Nestin, and Musashi-1. Although cell death was not significantly triggered by the eckol treatment at the concentration range 10 to $80 \mu \mathrm{M}$, there was an increase of cell kill rate noted after application of eckol in concentration $90 \mu \mathrm{M}$. Eckol treatment led to a marked reduction of the Sox2 levels in glioma-initiating cells. Sox2 is a transcription factor essential for maintaining the self-renewal of several types of undifferentiated stem cells, in particular, neural stem cells. Sox2 is also key component for maintaining self-renewal capacity of the brain cancer stem cells [192]. Eckol suppressed expression of Notch2 and $\beta$-catenin playing important role in the self-renewal potential regulation of the cancer stem-like cells in several human cancers. Treatment with eckol was also shown to decreases tumorigenic capacity. In other words, glioma cells treated with eckol lose their colony forming capacity in soft agar. Results of the in vivo experiments showed that eckol treatment contributes to marked tumor growth inhibition in xenograft mice [190]. Importantly, eckol treatment also effectively reduced the resistance of the glioma stem-like cells to ionizing radiation and temozolomide.

It is also known that the key signaling pathways PI3K-Akt and Ras-Raf-1-Erk activated in cancer stem-like cells are involved in the survival and maintaining the stemness in cancer stem-like cells. PI3K-Akt pathway contributes to the resistance of cancer cells to ionizing radiation as well [193]. Furthermore, Akt signaling pathway regulates the function of $\mathrm{ABCG} 2$, which is one of the $\mathrm{ABC}$ transporters related to the multi-drug resistant genes. Also, Akt regulates ABCG2 localization in the plasma membrane, thereby making them drug resistant. Hyun et al. have discovered that eckol treatment effectively inhibits both PI3K-Akt and Ras-Raf-1-Erk pathways in the glioma stem-like cells. Treatment with eckol was also noted to induce a marked suppression of the PI3K and Akt activities, and completely inhibit Ras-Raf-1 interaction and Raf-1 and Erk activations in the sphere-forming glioma stem-like cells. On the base of presented results, authors made a conclusion that eckol may enhance the sensitivity of glioma stem-like cells to anticancer therapies such as ionizing radiation or chemical drugs via inhibition of PI3K-Akt and Ras-Raf-1-Erk pathways [190].

The synthesized phloroglucinol derivative 2,4-bis(4-fluorophenylacetyl)phloroglucinol (BFP) induced cell death and slowed proliferation rate in three glioma cell lines U251, U87 and C6 in a concentration-dependent manner. At the same time, it did not affect primary human astrocytes (Table 40) [194].

Treatment with BFP $(3 \mu \mathrm{M})$ for 4 hours induced a nuclear shrinkage and nuclear condensation. Nuclear fragmentation was noted after $8 \mathrm{~h}$ of treatment. BFP also induced concentration-dependent sub-G1 arrest in U251 human glioma cells. Furthermore, treatment of U251 cells with BFP induced significantly upregulated Bax protein expression but did 
not affect expression of Bcl-2 protein. BFP application also increased procaspase-3 degradation and caspase-3 cleaved form expression in glioma cells. Upstream procaspase- 9 also degraded and cleaved-caspase-9 increased upon BFP treatment in U251 cells. BFP also increased cleaved-PARP expression time-dependently. Treatment of U251 glioma cells with BFP induced generation of the reactive oxygen species (ROS) and also increased expression of the endoplasmic reticulum (ER) stress markers such glucose-regulated protein (GRP)-78, GRP-94, IRE1, phosphorylation of eukaryotic translation initiation factor 2 alpha (eIF-2) and induced up-regulation of the CAAT/enhancer-binding protein homologous protein $(\mathrm{CHOP})$. In addition, BFP treatment provoked down-stream caspase activation such as pro-caspase- 7 and procaspase- 12 degradation suggesting the induction of ER stress [194]. These results indicate that the phloroglucinol derivative BFP induces human glioma cell death via mediation of the ROS generation, which subsequently enhances GPR78 and CHOP expression, increases activity of caspase-9 and caspase-3 leading to apoptosis.

Table 40. Antiproliferative activity of the phloroglucinol derivative against glioma cells, U251, U87 and C6 [194].

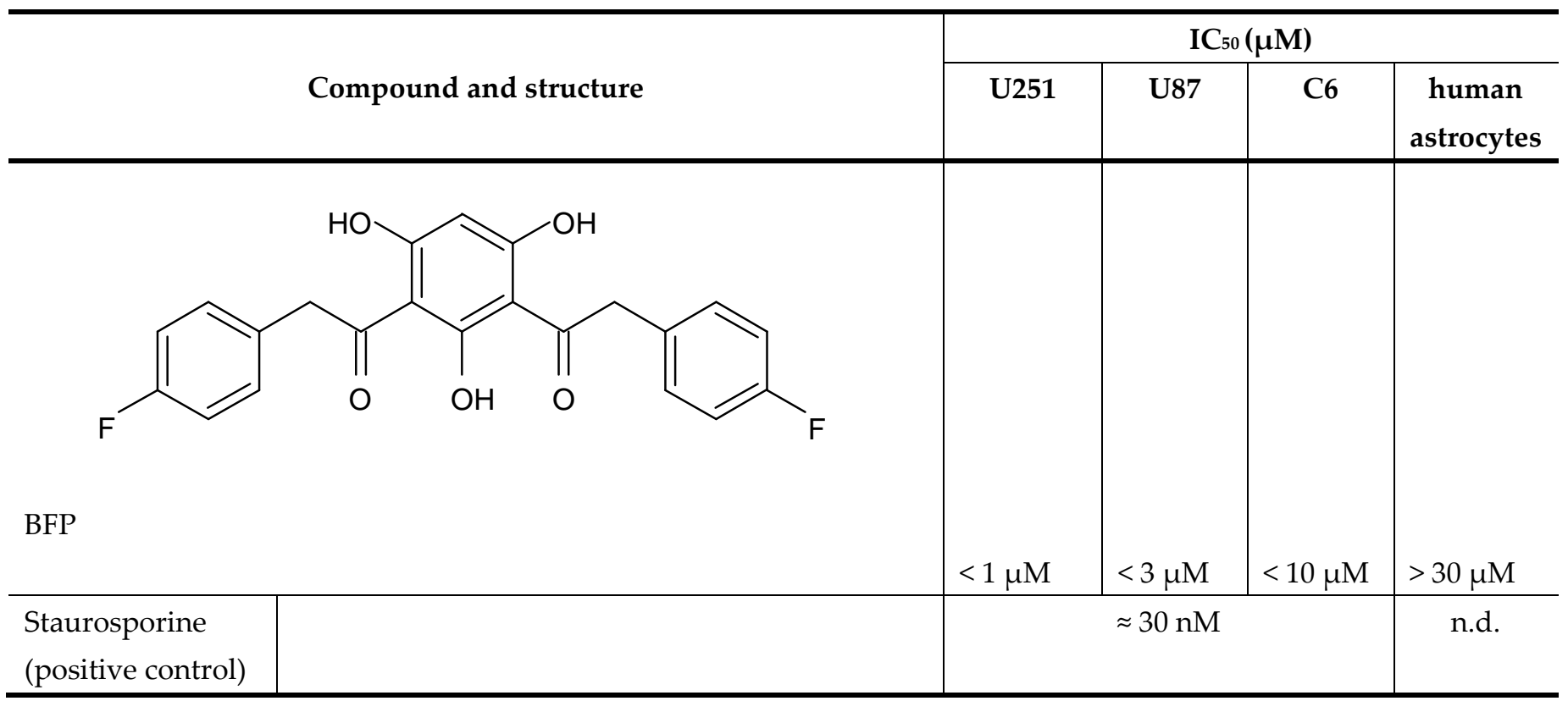

\section{Carotenoids}

Carotenoids along with chlorophylls and phycobiliproteins build a large group of organic pigments. They represent a class of tetraterpene pigments commonly found in bacteria, fungi, algae as well as high plants and animals. Nowadays, the number of carotenoid sources described is more than 700 . They have many biological functions referred in the literature including provitamin A capacity, antioxidant, anti-inflammatory, антипролиферативная, antihyperglyсаетіс, проапоптотическая, antibacterial and antiviral activities as well as upregulation of immune system [195]. Usually, carotenoids are classified into two main groups: (i) carotenes $(\alpha / \beta / \gamma / \delta$-carotene, lycopene) derived from hydrogen and carbon (pure hydrocarbons), and (ii) xanthophylls (astaxanthin, fucoxanthin, lutein) presenting oxygenated carotene compounds. The most known and studies carotenoids include fucoxanthin, astaxanthin, sifonaxanthin, violaxanthin, neoxanthin, $\beta$ carotene, capsanthin, lutein and others. Fucoxanthene is one of the post promising among then and one of the most common in culture, in particular marine environment containing more them $10 \%$ total production of carotenoids in nature [196].

Fucoxanthin. Fucoxanthin is an orange pigment possessing unique structure that contains allenic bond, conjugated carbonyl, 5,6- monoepoxide, and acetyl groups (Figure 
21) [197]. This compound is produced by both microalgae Bacillariophyta and macroalgae Phaeophyceae and it functions as an antenna pigment carotenoid coupled to the thylakoid membrane in the light-harvesting complexes and it transfers energy to the photosynthetic electron transport chain. Besides, it is can be extracted from almost all photosynthetic and nonphotosynthetic organisms such as bacteria and fungi [198].

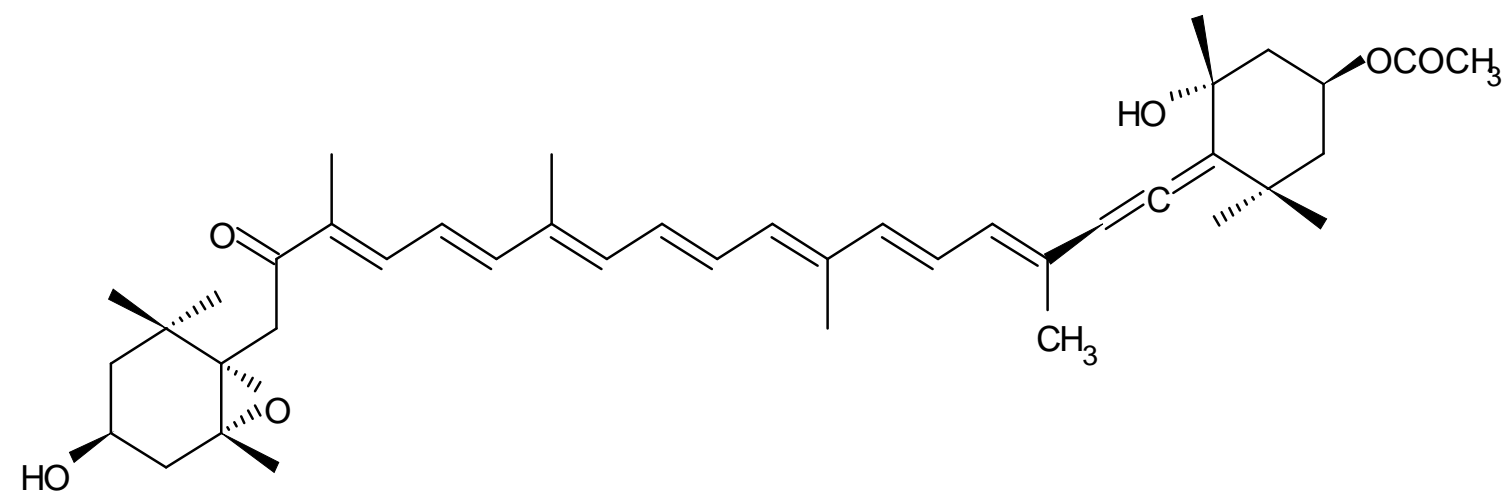

Figure 21. Chemical structure of fucoxanthin derived from marine brown algae

Seaweeds are rich with fucoxanthin, which is considered as effective anticancer, antioxidant, antiangiogenic, antidiabetic, antiobese, anti-inflammatory, and photo-protection pharmacological agent due to its powerful antioxidant properties [198]. Antiproliferative and antiapoptosis effects of fucoxanthin were confirmed in the human cancer cell lines including hepatic carcinoma HepG2, gastric adenocarcinoma MGC-803, and nonsmall-cell lung cancer cells [199].

Regarding the effects of fucoxanthin on the glioma cell growth, it was shown that fucoxanthin in concentrations $25,50,75$ и $100 \mu \mathrm{M}$ significantly reduced cell viability in the glioma U87 and U251cell lines in dose-dependent and time-dependent manner, but did not affect viability of healthy neurons. Applied in concentrations 25 and $50 \mu \mathrm{M}$ it induced apoptosis due to the disruption of mitochondrial potential $(\Delta \psi \mathrm{m})$ and destroyed mitochondrial function. It was also found that fucoxanthin provides an increment in Bax expression and a decrement in Bcl-2 expression in both U87 and U251 cells [200]. The balance of Bcl-2/Bax dominates the switch which turns on or off the apoptosis. Enhanced activation of cleaved-PARP, caspase- 9 and caspase- 3 was noted in the fucoxanthin-treated tumor cells. Electron microscopy studies had confirmed that fucoxanthin promotes cell death by induction apoptosis via mitochondrial pathway. It was further figured out that expression of phosphorylation in Akt and mTOR was dramatically decreased by fucoxanthin treatment of the glioma cells in concentration of 25 and $50 \mu \mathrm{M}$. Moreover, the level of phosphorylated mTOR in the glioma cells was notably reduced at higher concentration of fucoxanthin. The expressions of active Akt/mTOR form were lessened due to the glioma cell exposure with fucoxanthin or PI3K inhibitor, LY294002, individually.

In was shown in the scratch wound healing assay and trans-well assays that fucoxanthin can inhibit invasion and migration rates of the human glioblastoma cells. It was found out also that fucoxanthin possess a capacity of human glioblastoma cell inhibition and the protein levels of matrix metalloproteases (MMPs), MMP-2 and MMP-9, is significantly suppressed after incubation of glioma cells with fucoxanthin at the concentration of 25 and $50 \mu \mathrm{M}$. It is also known that MMPs-caused invasion and migration of the cancer cells are mediated by the family of the mitogen-activated protein kinases (MAPKs). There are three classes of MAPK, known such as extracellular signal-regulated kinases (ERK), stressactivated JUN N-terminal kinases (JNKs), and p38. The degree of the phosphorylated-p38 expression was significantly decreased in a concentration-dependent manner after treatment of the cells with fucoxanthin. However, fucoxanthin abnormally increased the protein levels of phosphorylated ERK and did not affect expression of p-JNK/JNK. In addition, the MMPs was significantly reduced by the p38 inhibitor (SB203580) treatment. 
Results of the tumor xenograft studies in the immune compromised nude mice (BALB/cnude) used as a test model had shown that tumor volume and weight were obviously lowered that demonstrate the tumor growth inhibiting capacity of fucoxanthin in U87 cells. Such molecules as p-Akt, p-mTOR, p-p38, MMP-2/9 and BCL-2 were all down-regulated, whereas Bax and cleaved-caspases- 9 were up-regulated after fucoxanthin treatment of the tumor tissues. These findings were consistent with in vitro results [200]. Therefore, there are convincing experimental evidences indicating that fucoxanthin exerts antiglioma activity, promotes apoptosis via PI3K/Akt/mTOR pathway inhibition and suppresses tumor invasion and migration due to the restriction of the p38/MMPs signaling pathway in human glioblastoma cells. Authors of the research studies suppose that fucoxanthin may represent a new anticancer drug prototype that can damage glioma cells and prevent metastasis. It may find its way as an emerging therapeutic agent in the future.

Hydratoperidinin. Carotenoid hydratoperidinin (molecular formula C39H52O8) isolated from the sea anemone Anthopleura midori showed moderate antiproliferative activity against rat glioma C6 and human glioma U251 cells [111]. Antitumor activity of hydratoperidinin was significantly higher than temozolomide activity (Table 41).

Table 41. Anti-glioma activity of the hydratoperidinin from the sea anemone Anthopleura midori [111].

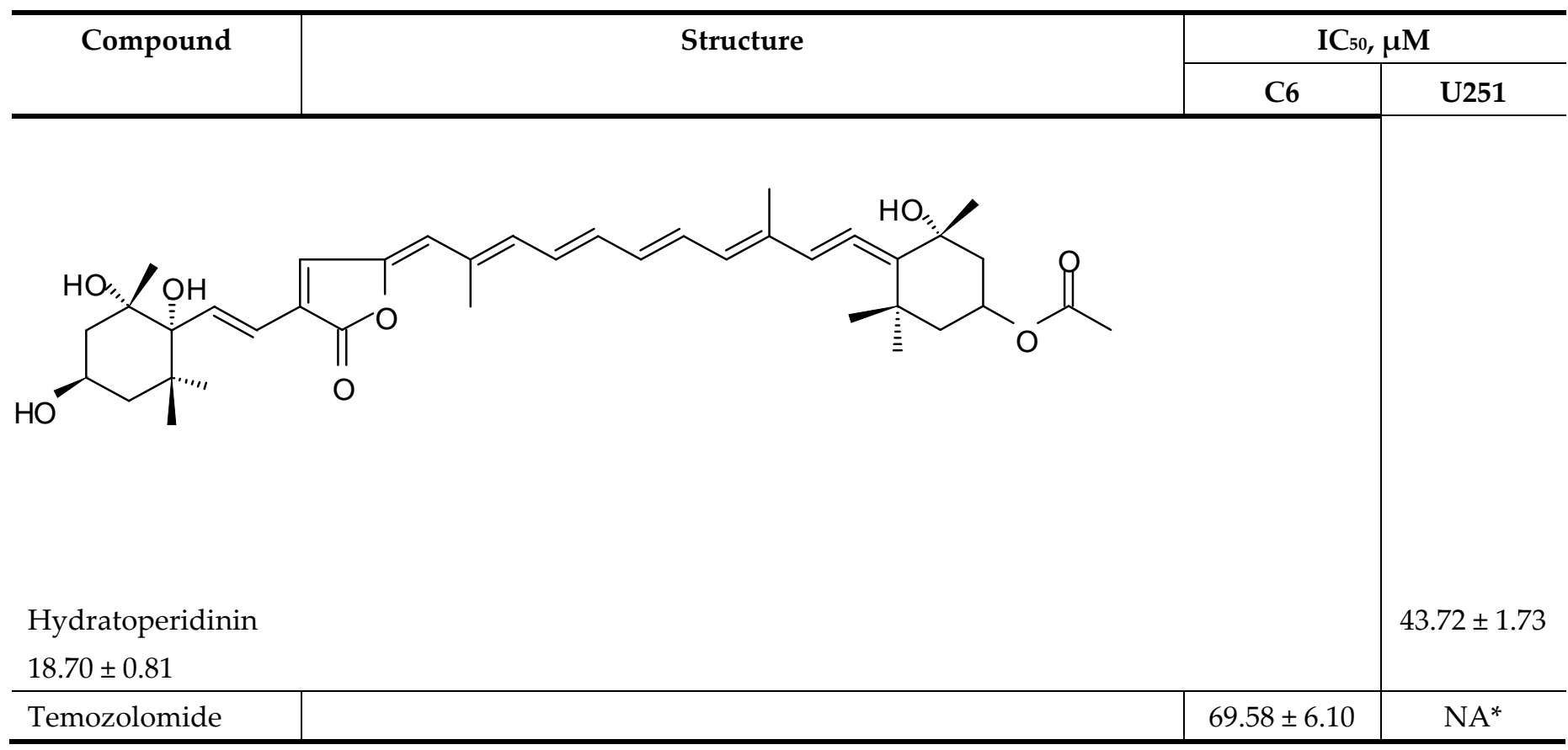

* NA: no inhibitory activity at concentration of $100 \mu \mathrm{M}$.

\section{Conclusion}

Quantitative analysis of the data presented in the previous sections of this review shows that more than a hundred of structurally diverse marine natural compounds possess significant capacity of suppressing proliferative activity of the various glioma cell lines and often even reduce their viability. Some of those compounds are capable to inhibit activity of the cancer cells themselves as well as the tumor stem cells. Authors of the experimental investigations suppose that those compounds may be considered as prototypes for the development of novel anti-glioma pharmaceuticals. In the most studies average inhibiting concentrations of the tested compounds under in vitro conditions were comparable with cytotoxic concentration of antitumor drugs including temozolomide, which is the first line drug of the standard antiglioblastoma chemotherapy. A group of compounds such as ecteinascidin-770, renieramycin $M$, papuamine, actinomycins $D, V$ 
and $X 0 \beta$, fradimycin B, valinomycin, streptodepsipeptides P11A and P11B had demonstrated extremely high cytotoxicity inducing glioma cell death in the nanomolar concentration range where half inhibiting concentrations of the officinal antitumor drugs are usually in the range between a few micromoles and several dozen micromoles. For example, IC50 for temozolomide is approximately 85.8 and $69.5 \mu \mathrm{M}$ for the glioma cell lines SHG-44 and C6, respectively, and more than $100 \mu \mathrm{M}$ for U87-MG and U251 cells [113]. At the same time, phorboxazoles isolated from marine sponges and exerted antiglioma effects in nanomolar concentrations have a property of the most potent cytostatic agents among all pharmaceuticals studied so far [187]. Despite cytotoxic activity is not the only parameter of the anticancer potential of chemical compounds, we would like to point out that the substances with the IC50 values greater than 100-150 $\mu \mathrm{M}$ rather do not have a perspective as the potential anti-glioma drugs.

Important factor for the successful development of natural compounds towards novel anti-glioma agents is an index of selectivity degree of compounds regarding cancer cells. Usually, it is expressed as a ratio of IC50 for the normal cells (for example, for astrocytes, fibroblasts or HUVEC) to the IC50 for glioma cells. Unfortunately, this parameter was provided only in few numbers of publications. Nevertheless, it should be mentioned that such compounds pheophorbide a isolated from read algae [184], triterpene and steroid glycosides from the starfish and holothurians [103,110], sesquiterpene aplysin discovered in gastropod molluscs [120], anthracycline antibiotic SZ-685C from marine fungi [97] as well as macrolide antibiotics flavofungin II and spectinabilin isolated from marine streptomyces bacteria выделенные из морских бактерий стрептомицетов [102] demonstrated substantial inhibition of the glioma cells along with mild toxic influence on the normal cells. At the same time, a series of compounds such as, for example, meriolins and fascaplysins apart of the extremely strong antiglioma activity exert pronounced toxic effect on the normal cells $[51,64]$. Structures of such compounds can be considered only as a base for further discovery of the new agents that would be less toxic for the normal cells.

Results of analysis of the taxonomical diversity of the marine species that were used as a source of the compounds with anti-glioma activity pose some interest (Table 42). Despite an enormous list of those compounds, about half of the active compounds were isolated from a few actinomyces bacterial strains belonging to the one genus Streptomyces (about 40 compounds) and from marine sponges (about 20 compounds) belonging to the class Demospongiae (phylum Porifera). Also some significant number of those compounds were isolated from star fish (class Asteroidea), sea cucumbers (class Holothuroidea) and ascidians (phylum Chordata, subphylum Tunicata). Compounds exerting anti-glioma activity in small amounts was found in cyanobacteria, marine fungi, red and brown alga, actinians (sea anemones), crustaceans, and mollusks. Therefore, now we have a substantially limited number of taxons that were marked with the presence of anti-glioma activity. That does not mean that the further search of active compounds should be limited with only those taxons. That is the opposites, taking to account the fact that taxonomical diversity of the marine species is substantially greater than the terrestrial species diversity. Also, chemical diversity of the marine compounds is probably exceeding the one of terrestrial species. Therefore, it would be reasonable to expand the search of the anticancer agents in representatives of other marine taxons.

Table 42. Taxonomy of marine organisms containing bioactive compounds with experimentally confirmed anti-glioma activity (classification is presented in accordance with World Register of Marine Species).

\begin{tabular}{cc}
\hline Species*/strain & Anti-glioma compound \\
\hline Kingdom Bacteria \\
Phylum Actinobacteria \\
Class Actinobacteria \\
Order Actinomycetales \\
Family Streptomycetaceae \\
\hline
\end{tabular}




\begin{tabular}{|c|c|c|}
\hline $\begin{array}{l}\text { Streptomyces } \\
\text { ZZ406 }\end{array}$ & $\begin{array}{l}\text { Anthraquinones: 1-hydroxymethyl-8-hydroxy-anthraquinone-3-carboxylic acid, } \\
\text { chrysophanol, 3,8-dihydroxy-1-methyl-anthraquinone-2-carboxylic acid. } \\
\text { Chromon phaeochromycin I. }\end{array}$ & {$[143]$} \\
\hline $\begin{array}{l}\text { Streptomyces sp. P11- } \\
\text { 23B }\end{array}$ & $\begin{array}{l}\text { Cyclodepsipeptides: } \quad \text { valinomycin, } \quad \text { streptodepsipeptide } \\
\text { streptodepsipeptide P11B. }\end{array}$ & [137] \\
\hline $\begin{array}{l}\text { Streptomyces } \\
\text { ZQ4BG }\end{array}$ & Antibiotics macrolides: flavofungin I, flavofungin II, spectinabilin. & {$[102]$} \\
\hline Streptomyces sp. Q22 & Bagremycin antibiotics: bagremycins B, C. & {$[98]$} \\
\hline $\begin{array}{l}\text { Streptomyces } \\
\text { 182SMLY }\end{array}$ & Polycyclic quinones: N-acetyl-N-demethylmayamycin, streptoanthraquinone A. & {$[145]$} \\
\hline $\begin{array}{l}\text { Streptomyces } \\
\text { ZZ338 }\end{array}$ & Actinomycin antibiotics: actinomycin $\mathrm{D}$, actinomycin $\mathrm{V}$, actinomycin $\mathrm{X}_{0 \beta}$. & [94] \\
\hline $\begin{array}{l}\text { Streptomyces fradiae } \\
\text { PTZ0025 }\end{array}$ & Capoamycin-type antibiotics: fradimycin A, fradimycin B, MK844-mF10. & {$[100]$} \\
\hline $\begin{array}{l}\text { Streptomyces } \\
\text { antibioticus H12-15 }\end{array}$ & Antimycin antibiotics: neoantimycins A, B, antimycins A1ab, A2a, A9. & [96] \\
\hline $\begin{array}{l}\text { Streptomyces anandii } \\
\text { H41-59 }\end{array}$ & New ergosterols: ananstreps A, B, C, ten known ergosterols. & {$[141]$} \\
\hline
\end{tabular}

\section{Phylum Firmicutes \\ Class Bacili \\ Order Bacillales \\ Family Bacillaceae}

Bacillus sp. FS8D Alkaloid pseurotin A

\section{Phylum Cyanobacteria \\ Class Cyanophyceae \\ Order Synechococcales \\ Family Leptolyngbyaceae}

Leptolyngbya sp. Cyclopeptide coibamide A

\section{Kingdom Fungi}

Phylum (Division) Ascomycota

Class Sordariomycetes

Order Xylariales

Family Xylariaceae

\begin{tabular}{ll}
\hline Xylaria sp. (No. 2508) & Xyloketal B \\
$\begin{array}{l}\text { Halorosellinia sp. (No. } \\
1403 \text { ) }\end{array}$ & Anthracycline antibiotic SZ-685C \\
& Class Eurotiomycetes \\
& Order Eurotiales \\
& Family Trichocomaceae
\end{tabular}

Penicillium sp. ZZ380 Alkaloids: pyrrospirones C-J, penicipyrroether A

Order: incertae sedis

Trichobotrys effuse Alkaloid trichobamide A 


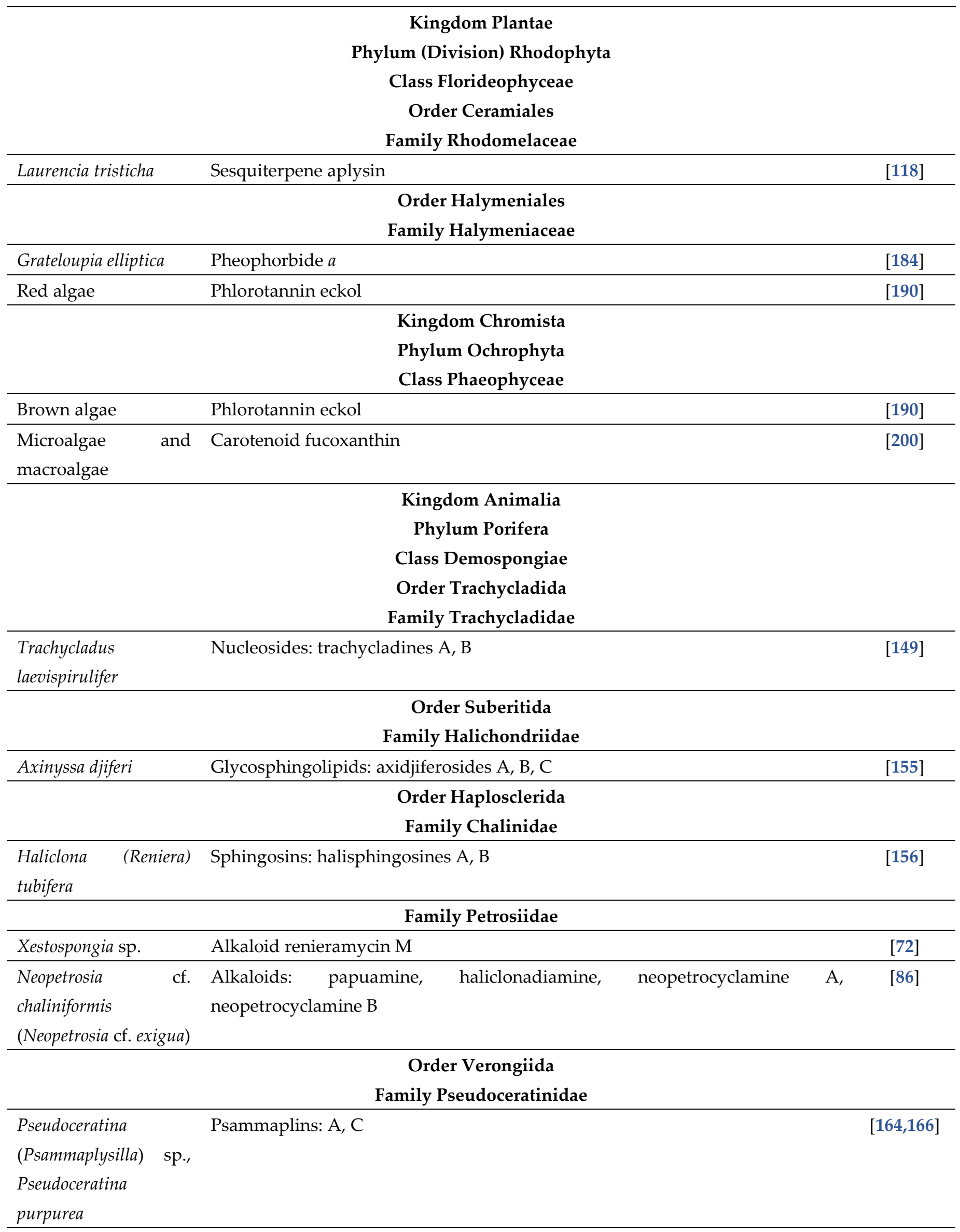




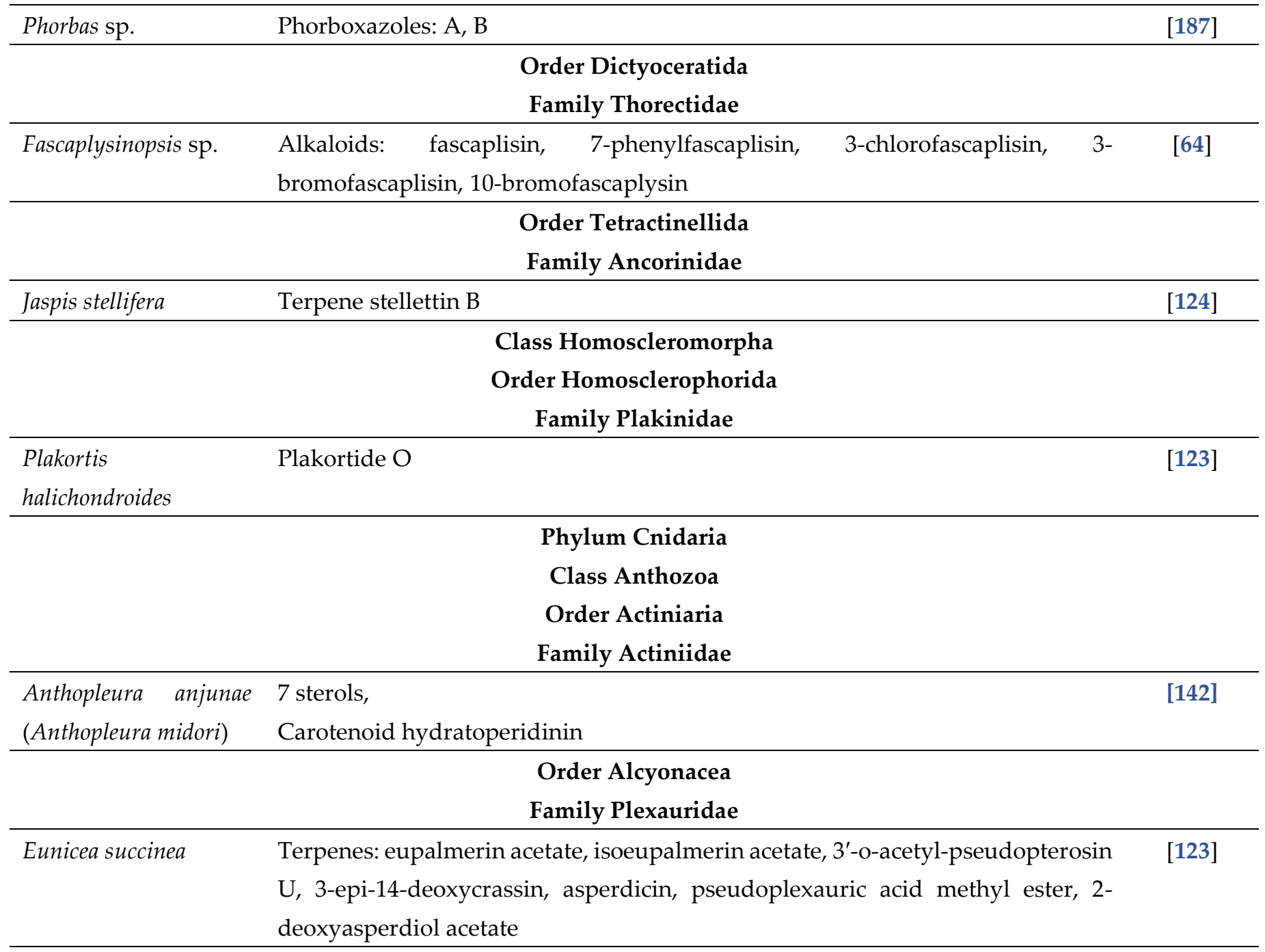

\section{Phylum Echinodermata \\ Class Crinoidea \\ Order Comatulida \\ Family Comatulidae}

Comanthus AH Clark Anthraquinone derivatives: 1'-deoxyrhodoptilometrin, (S)-(-)-rhodoptilometrin

(Comanthus) sp.

\section{Class Asteroidea \\ Order Valvatida \\ Family Oreasteridae}

\begin{tabular}{|c|c|c|}
\hline \multirow[t]{3}{*}{ Culcita novaeguineae } & Steroidal saponin asterosaponin 1 & [104] \\
\hline & 4 steroidal saponins; & [103] \\
\hline & Polyhydroxysteroidal glycosides: culcinosides A, B, C, D & [105] \\
\hline $\begin{array}{l}\text { Anthenea pentagonula } \\
\text { (Anthenea chinensis) }\end{array}$ & Polyhydroxysteroidal glycoside anthenoside A & {$[106]$} \\
\hline Pentaceraster chinensis & 2 steroidal glycosides & [108] \\
\hline \multicolumn{3}{|c|}{ Family Ophidiasteridae } \\
\hline Narcissia canariensis & Glycosphingolipids: ophidiacerebroside B, C, D & [154] \\
\hline
\end{tabular}

\section{Order Paxillosida}




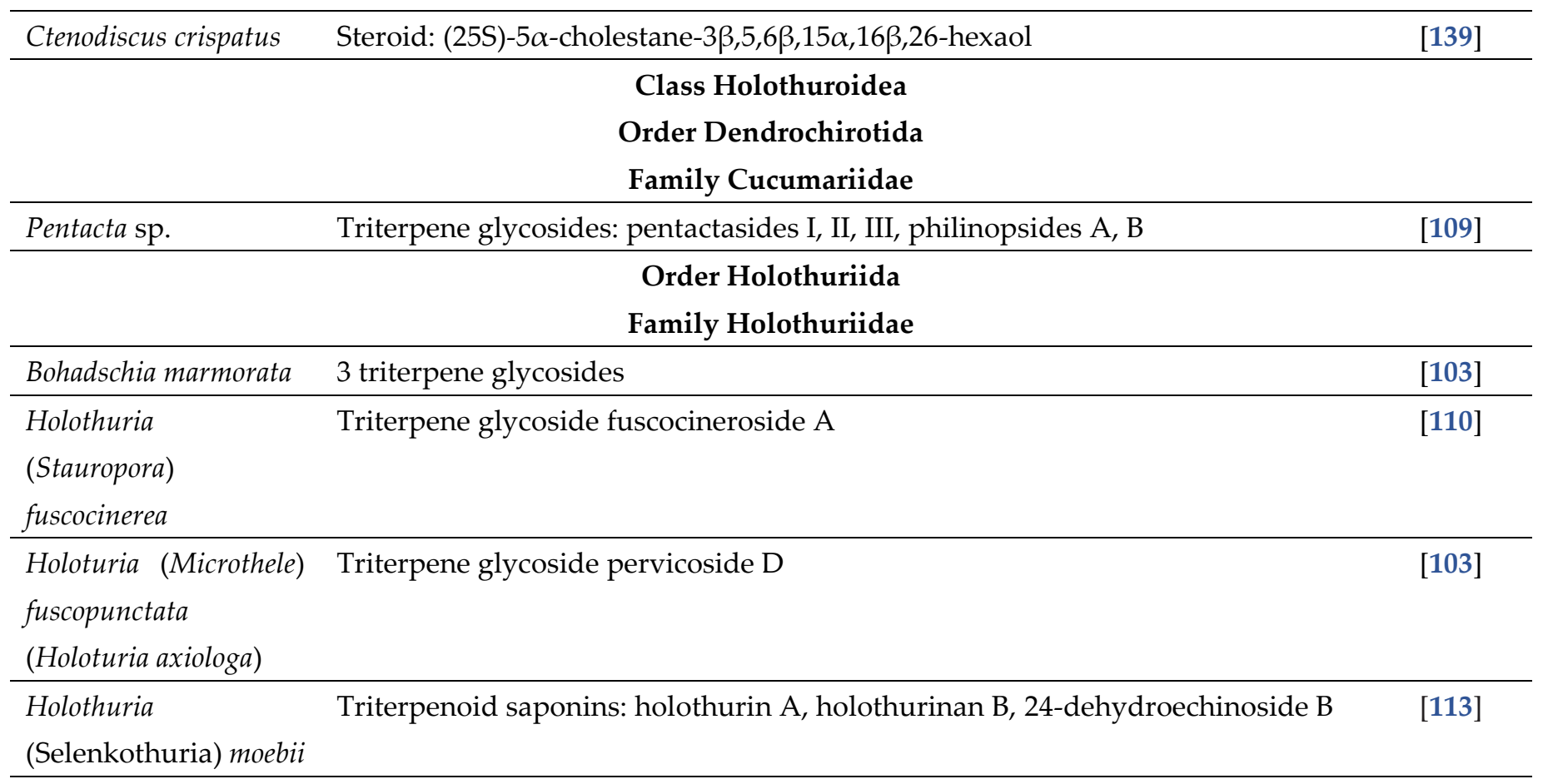

\section{Phylum Arthropoda \\ Subphylum Chelicerata \\ Class Merostomata \\ Order Xiphosurida \\ Family Limulidae}

Tachypleus tridentatus Tachyplesin I

$[130,131]$

\section{Phylum Mollusca \\ Class Gastropoda \\ Order Aplysiida \\ Family Aplysiidae}

\begin{tabular}{cc}
\hline Aplysia kurodai & Terpene aplysin \\
\hline & Phylum Chordata \\
& Subphylum Tunicata \\
& Class Ascidiacea \\
& Order Stolidobranchia \\
& Family Styelidae
\end{tabular}

Polyandrocarpa Alkaloids: zorrimidazolone, 3-indolylglyoxylic acid, 3-indolylglyoxylic aci

zorritensis methyl ester

Dendrodoa

grossularia

Polycarpa aurata $\quad$ Alkaloids $\alpha$-carbolines

\section{Order Phlebobranchia}

Family Perophoridae

Ecteinascidia turbinata Alkaloids ecteinascidin-770, 2'-N-4"'-pyridinecarbonyl derivative of ET-770

\section{Order Aplousobranchia}

Family Didemnidae

Didemnum granulatum Alkaloids: granulatimide, isogranulatimide, 
2 amino analogs.

Eudistoma cf. glaucum Alkaloids: 7 synthetic analogues of rigidins

(Eudistoma cf. rigida)

** Taxons in parentheses are synonyms.

Funding: This work was supported by Ministry of Science and Higher Education of the Russian Federation (Project \#0657-2020-0004).

Data Availability Statement: All the data provided in the review article

Conflicts of Interest: The authors declare no conflict of interest.

\section{References}

1. Perry, A.; Wesseling, P. Histologic classification of gliomas. Handb Clin. Neurol. 2016, 134, 71-95.

2. Otani, R.; Uzuka, T.; Ueki, K. Classification of adult diffuse gliomas by molecular markers - a short review with historical footnote. Jap. J. Clin. Oncol. 2017, 47, 2-6.

3. Wesseling, P.; Capper, D. WHO 2016 classification of gliomas. Neuropathol. Appl. Neurobiol. 018, 44, $139-150$.

4. Ostrom, Q.T.; Gittleman, H.; Truitt, G.; Boscia, A.; Kruchko, C.; Barnholtz-Sloan, J.S. CBTRUS statistical report: primary brain and other central nervous system tumors diagnosed in the United States in 2011-2015. Neuro-oncology 2018, $20,1-86$.

5. Zreik, J.; Moinuddin, F.M.; Yolcu, Y.U.; Alvi, M.A.; Chaichana, K.L.; Quinones-Hinojosa, A.; Bydon, M. Improved 3-year survival rates for glioblastoma multiforme are associated with trends in treatment: analysis of the national cancer database from 2004 to 2013. J. Neurooncol. 2020, 148, 69-79.

6. Zhang, J.; Stevens, M.F.; Bradshaw, T.D. Temozolomide: mechanisms of action, repair and resistance. Curr. Mol. Pharmacol. 2012, 5, 102-114.

7. Erasimus, H.; Gobin, M.; Niclou, S.; Van Dyck, E. DNA repair mechanisms and their clinical impact in glioblastoma. Mutat. Res. Rev. Mutat. Rev. 2016, 769, 19-35.

8. Van Thuijl; H.F., Mazor, T.; Johnson, B.E.; Fouse, S.D.; Aihara, K.; Hong, C.; Malmstrom, A. Hallbeck, M.; Heimans, J.J.; Kloezeman, J.J. Evolution of DNA repair defects during malignant progression of low-grade gliomas after temozolomide treatment. Acta Neuropathol. 2015, 129, 597-607.

9. Goellner, E.M.; Grimme, B.; Brown, A.R.; Lin, Y.C.; Wang, X.H.; Sugrue, K.F.; Mitchell, L.; Trivedi, R.N.; Tang, J.-B.; Sobol, R.W. Overcoming temozolomide resistance in glioblastoma via dual inhibition of NAD+ biosynthesis and base excision repair. Cancer Res. 2011, 71, 2308-2317.

10. Ferreira, J.; Ramos, A.A.; Almeida, T.; Azqueta, A.; Rocha, E. Drug resistance in glioblastoma and cytotoxicity of seaweed compounds, alone and in combination with anticancer drugs: A mini review. Phytomedicine 2018, 48, 84-93.

11. Tait, S.W.G.; Ichim, G.; Green, D.R. Die another way - non-apoptotic mechanisms of cell death. J. Cell Sci. 2014, 127, 21352144.

12. Jakubowicz-Gil, J.; Langner, E.; Badziul, D.; Wertel, I., Rzeski, W. Apoptosis induction in human glioblastoma multiforme T98G cells upon temozolomide and quercetin treatment. Tumour Biol. 2013, 34, 2367-2378.

13. Bae, S.H.; Park, M.-J.; Lee, M.M.; Kim, T.M.; Lee, S.-H.; Cho, S.Y.; Kim, Y.-H.; Kim, Y.J.; Park, C.-K.; Kim, C.-Y. Toxicity profile of temozolomide in the treatment of 300 malignant glioma patients in Korea. J. Korean Med. Sci. 2014, 29, 980-984.

14. Signore, M.; Pelacchi, F.; di Martino, S.; Runci, D.; Biffoni, M.; Giannetti, S.; Morgante, L.; De Majo, M.; Petricoin, E.F.; Stancato, L.; Larocca, L.M.; De Maria, R.; Pallini, R.; Ricci-Vitiani, L. Combined PDK1 and CHK1 inhibition is required to kill glioblastoma stem-like cells in vitro and in vivo. Cell Death Dis. 2014, 5, e1223.

15. Aldape, K.; Nejad, R.; Louis, D.N.; Zadeh, G. Integrating molecular markers into the World Health Organization classification of CNS tumors: a survey of the neuro-oncology community. Neuro-oncology 2017, 19, 336-344. 
16. Chen, J.; McKay, R.M.; Parada, L.F. Malignant glioma: lessons from genomics, mouse models, and stem cells. Cell 2012, 149, $36-47$.

17. Maleszewska, M.; Kaminska, B. Is glioblastoma an epigenetic malignancy? Cancers 2013, 5, 1120-1139.

18. Clarke, J.; Butowski, N.; Chang, S. Recent advances in therapy for glioblastoma. Arch. Neurol. 2010, 67, $279-283$.

19. Parsons, D.W.; Jones, S.; Zhang, X., Lin, J.C.-H.; Leary, R.J.; Angenendt, P.; Mankoo, P.; Carter, H.; Siu, I.-M.; Gallia, G.L. An integrated genomic analysis of human glioblastoma multiforme. Science 2008, 321, 1807-1812.

20. Fan, Q.W.; Weiss, W.A. Targeting the RTK-PI3K-mTOR axis in malignant glioma: overcoming resistance. Curr. Top. Microbiol. Immunol. 2010, 347, 279-296.

21. Gong, A.; Gong, L.; Yao, W.; Ge, N.; Lu. L.; Liang, H. Aplysin induces apoptosis in glioma cells through HSP90/AKT pathway. Exp. Biol. Med. 2015, 240, 639-644.

22. Shi, L.; Chen, J.; Wang Y.-Y.; Sun, G.; Liu J.-N.; Zhang, J.-X.; Yan, W.; Qian, C.-F.; Liu, N.; Fu, Z.; You, Y.-P.; Zeng, Y. Gossypin induces G2/M arrest in human malignant glioma U251 cells by the activation of Chk1/Cdc25C pathway. Cell. Mol. Neurobiol. 2012, 32, 289-296.

23. Qiu, Z.; Oleinick, N.L.; Zhang J. ATR/CHK1 inhibitors and cancer therapy. Radiother. Oncol. 2018, 126, 450-64.

24. Qiu, Z.; Fa, P.; Liu, T.; Prasad, C.B.; Ma, S.; Hong, Z.; Chan, E.R.; Wang, H.; Li, Z.; He, K.; Wang, Q.-E.; Williams, T.M.; Yan, C.; Sizemore, S.T.; Narla, G.; Zhang, J. A genome-wide pooled shRNA screen identifies PPP2R2A as a predictive biomarker for the response to ATR and CHK1 inhibitors. Cancer Res. 2020, 80, 3305-3318.

25. Kessler, R.; Bleichert, F.; Warnke, J.-P.; Eschrich, K. 6-Phosphofructo-2-kinase/fructose-2,6- bisphosphatase (PFKFB3) is upregulated in high grade astrocytomas. J. Neurooncol. 2008, 86, 257-264.

26. Kefas, B.; Comeau, L.; Erdle, N.; Montgomery, E.; Amos, S.; Purow, B. Pyruvate kinase M2 is a target of the tumorsuppressive microRNA-326 and regulates the survival of glioma cells. Neuro-oncology 2010, 12, 1102-1112.

27. Wolf, A.; Agnihotri, S.; Micallef, J.; Mukherjee, J.; Sabha, N.; Cairns, R.; Hawkins, C.; Guha, A. Hexokinase 2 is a key mediator of aerobic glycolysis and promotes tumor growth in human glioblastoma multiforme. J. Exp. Med. 2011, 208, 313-326.

28. Seliger, C.; Leukel, P.; Moeckel, S.; Jachnik, B.; Lottaz, C.; Kreutz, M.; Brawanski, A.; Proescholdt, M.; Bogdahn, U.; Bosserhoff, A.-K. Lactate-modulated induction of THBS-1 activates transforming growth factor (TGF)-beta2 and migration of glioma cells in vitro. PLoS One 2013, 8, e78935.

29. Newman, D.J.; Cragg, G.M. Natural products as sources of new drugs from 1981 to 2014. J. Nat. Prod. $2016,79,629-661$.

30. Khalifa, S.A.M.; Elias, N.; Farag, M.A.; Chen, L.; Saeed, A.; Hegazy, M.-E.F.; Moustafa, M.S.; Abd El-Wahed, A.; Al-Mousawi, S.M.; Musharraf, S.G. Marine natural products: a source of novel anticancer drugs. Mar. Drugs 2019, $17,491$.

31. Altmann, K.-H. Drugs from the oceans: marine natural products as leads for drug discovery. Chimia 2017, 71, 646-652.

32. Khotimchenko, Y. Pharmacological potential of sea cucumbers. Int. J. Mol. Sci. 2018, 19, 1342.

33. Khotimchenko, M.; Tiasto, V.; Kalitnik, A.; Begun, M.; Khotimchenko, R.; Leonteva, E.; Bryukhovetskiy, I.; Khotimchenko, Y. Antitumor potential of carrageenans from marine red algae. Carbohydr. Polym. 2020, 246, 116568.

34. Jiménez, C. Marine natural products in medicinal chemistry. ACS Med. Chem. Lett. 2018, 9, 959-961.

35. Dyshlovoy, S.; Honecker, F. Marine compounds and cancer: the first two decades of XXI century 2020. Mar. Drugs 2020, 18, 20.

36. Debnath, B.; Singh, W.S.; Das, M.; Goswami, S.; Singh, M.K.; Maiti, D.; Manna, K. Role of plant alkaloids on human health: A review of biological activities. Mater. Today Chem. 2018, 9, 56-72.

37. Souza, C.R.M.; Bezerra, W.P.; Souto, J.T. Marine alkaloids with anti-inflammatory activity: Current knowledge and future perspectives. Mar. Drugs 2020, 18, 147.

38. Tao, H.; Zuo, L.; Xu, H.; Li, C.; Qiao, G.; Guo, M.; Lin, X. Alkaloids as anticancer agents: a review of Chinese patents in recent 5 years. Recent Pat. Anticancer Drug Discov. 2020, 15, 2-13. 
39. Aiello, A.; Fattorusso, E.; Imperatore, C.; Irace, C.; Luciano, P.; Menna, M.; Santamaria, R.; Vitalone, R. Zorrimidazolone, a bioactive alkaloid from the non-indigenous Mediterranean stolidobranch Polyandrocarpa zorritensis. Mar. Drugs 2011, 9, 11571165.

40. Loukaci, A.; Guyot, M.; Chiaroni, A.; Riche, C. A new indole alkaloid from the marine tunicate Dendrodoa grossularia. J. Nat. Prod. 1998, 61, 519-522.

41. Davis, R.A.; Aalbersberg, W.; Meo, S.; Da Rocha, R.M.; Ireland, C.M. The isolation and synthesis of poly androcarpamines A and B. Two new 2-aminoimidazolone compounds from the Fijian ascidian, Polyandrocarpa sp. Tetrahedron 2002, 58, 3263-3269.

42. Franco, L.H.; Joffé, E.B.; Puricelli, L.; Tatian, M.; Seldes, A.M.; Palermo, J.A. Indole alkaloids from the tunicate Aplidium meridianum. J. Nat. Prod. 1998, 61, 1130-1132.

43. Perry, N.B.; Ettouati, L.; Litaudon, M.; Blunt, J.W; Munro, M.H.G.; Parkin, S.; Hope, H. Alkaloids from the Antarctic sponge Kirkpatrickia varialosa. Part 1: Variolin B, a new antitumour and antiviral compound. Tetrahedron 1994, 50, $3987-3992$.

44. Akue-Gedu, R.; Debiton, E.; Ferandin, Y.; Meijer, L.; Prudhomme, M.; Fabrice Anizon, F.; Moreau, P. Synthesis and biological activities of aminopyrimidyl-indoles structurally related to meridianins. Bioorg. Med. Chem. 2009, 17, 4420-4424.

45. Watters, D.J. Ascidian toxins with potential for drug development. Mar. Drugs 2018, 16, 162.

46. Bharate, S.B.; Yadav, R.R.; Battula, S.; Vishwakarma, R.A. Meridianins: marine-derived potent kinase inhibitors. Mini Rev. Med. Chem. 2012, 12, 618-631.

47. More, K.N.; Jang, H.W.; Hong, V.S.; Lee, J. Pim kinase inhibitory and antiproliferative activity of a novel series of meridianin C derivatives. Bioorg. Med. Chem. Lett. 2014, 24, $2424-2428$.

48. Simone, M.; Erba, E.; Damia, G.; Vikhanskaya, F.; Di Francesco, A.M.; Riccardi, R.; Bailly, C.; Cuevas, C.; Sousa-Faro, J.M.F.; D'Incalci, M. Variolin B and its derivate deoxy-variolin B: new marine natural compounds with cyclin-dependent kinase inhibitor activity. Eur. J. Cancer 2005, 41, 2366-2377.

49. Bettayeb, K.; Tirado, O.M.; Marionneau-Lambot, S.; Ferandin, Y.; Lozach, O.; Morris, J.C.; Mateo-Lozano, S.; Drueckes, P.; Schaechtele, C.; Kubbutat, M.H.G. Meriolins, a new class of cell death inducing kinase inhibitors with enhanced selectivity for cyclin-dependent kinases. Cancer Res. 2007, 67, 8325-8334.

50. Echalier, A.; Bettayeb, K.; Ferandin, Y.; Lozach, O.; Clement, M.; Valette, A.; Liger, F.; Marquet, B.; Morris, J.C.; Endicott, J.A. Meriolins (3-(pyrimidin4-yl)-7-azaindoles): synthesis, kinase inhibitory activity, cellular effects, and structure of a CDK2/cyclin A/meriolin complex. J. Med. Chem. 2008, 51, 737-751.

51. Jarry, M.; Lecointre, C.; Malleval, C.; Desrues, L.; Schouft, M.-T.; Lejoncour, V.; Liger, F. Impact of meriolins, a new class of cyclin-dependent kinase inhibitors, on malignant glioma proliferation and neo-angiogenesis. Neuro-oncology 2014, 16, 14841498.

52. Roll, D.M.; Ireland, C.M.; Lu, H.S.M.; Clardy, J. Fascaplysin, an unusual antimicrobial pigment from the marine sponge Fascaplysinopsis sp. J. Org. Chem. 1988, 53, 3276-3278.

53. Jimènez, C.; Quiñoà, E.; Adamczeski, M.; Hunter, L.M.; Crews, P.J. Novel sponge-derived amino-acids.12. Tryptophanderived pigments and accompanying sesterterpenes from Fascaplysinopis-reticulata. J. Org. Chem. 1991, 56, 3401-3410.

54. Mahale, S.; Bharate, S.B.; Manda, S.; Joshi, P.; Bharate, S.S.; Jenkins, P.R.; Vishwakarma, R.A.; Chaudhuri, B. Biphenyl-4carboxylic acid [2-(1H-Indol-3-yl)-ethyl]-methylamide (CA224), a nonplanar analogue of fascaplysin, inhibits cdk4 and tubulin polymerization: evaluation of in vitro and in vivo anticancer activity. J. Med. Chem. 2014, 57, 9658-9672.

55. Zhidkov, M.E.; Smirnova, P.A.; Tryapkin, O.A.; Kantemirov, A.V.; Khudyakova, Y.V.; Malyarenko, O.S.; Ermakova, S.P.; Grigorchuk, V.P.; Kaune, M.; von Amsberg, G. et al. Total syntheses and preliminary biological evaluation of brominated fascaplysin and reticulatine alkaloids and their analogues. Mar. Drugs 2019, 17, 496.

56. Lin, J.; Yan, X.J.; Chen, H.M. Fascaplysin, a selective CDK4 inhibitor, exhibit anti-angiogenic activity in vitro and in vivo. Cancer Chemother. Pharmacol. 2007, 59, 439-445.

57. Hamilton, G. Cytotoxic effects of fascaplysin against small cell lung cancer cell lines. Mar. Drugs 2014, 12, 1377-1389. 
58. Oh, T.-I.; Lee, Y.-M.; Nam, T.-J.; Ko, Y.-S.; Mah, S.; Kim, J.; Kim, Y.; Reddy, R.H.; Kim, J.; Hong, S. et al. Fascaplysin exerts anticancer effects through the downregulation of survivin and HIF-1 $\alpha$ and inhibition of VEGFR2 and TRKA. Int. J. Mol. Sci. 2017, $18,2074$.

59. Rath, B.; Hochmair, M.; Plangger, A.; Hamilton, G. Anticancer activity of fascaplysin against lung cancer cell and small cell lung cancer circulating tumor cell lines. Mar. Drugs 2018, 16, 383.

60. Wang, F.; Chen, H.; Yan, X.; Zhen, Y. Fascaplysin sensitizes cells to TRAIL-induced apoptosis through upregulating DR5 expression. Chin. J. Oceanol. Limnol. 2013, 31, 560-569.

61. Kumar, S.; Guru, S.K.; Pathania, A.S.; Manda, S.; Kumar, A.; Bharate, S.B.; Vishwakarma, R.A.; Malik, F.; Bhushan, S. Fascaplysin induces caspase mediated crosstalk between apoptosis and autophagy through the inhibition of $\mathrm{PI} 3 \mathrm{~K} / \mathrm{AKT} / \mathrm{mTOR}$ signaling cascade in human leukemia HL-60 cells. J. Cell. Biochem. 2015, 116, 985-997.

62. Sharma, S.; Guru, S.K.; Manda, S.; Kumar, A.; Mintoo, M.J.; Prasad, V.D.; Sharma, P.R.; Mondhe, D.M.; Bharate, S.B.; Bhushan, S. A marine sponge alkaloid derivative 4-chloro fascaplysin inhibits tumor growth and VEGF mediated angiogenesis by disrupting PI3K/Akt/mTOR signaling cascade. Chem. Biol. Interact. 2017, 275, 47-60.

63. Bryukhovetskiy, I.; Lyakhova, I.; Mischenko, P.; Milkina, E.; Zaitsev, S.; Khotimchenko, Y.; Bryukhovetskiy, A.; Polevshchikov, A.; Kudryavtsev, I.; Khotimchenko, M. et al. Alkaloids of fascaplysin are effective conventional chemotherapeutic drugs, inhibiting the proliferation of C6 glioma cells and causing their death in vitro. Oncol. Lett. 2017, 13, $738-746$

64. Lyakhova, I.A.; Bryukhovetsky, I.S.; Kudryavtsev, I.V.; Khotimchenko, Y.S.; Zhidkov, M.E.; Kantemirov, A.V. Antitumor activity of fascaplysin derivatives on glioblastoma model in vitro. Bull. Exp. Biol. Med. 2018, 164, 666-672.

65. Mahale, S.; Bharate, S.B.; Manda, S.; Joshi, P.; Jenkins, P.R.; Vishwakarma, R.A. Antitumor potential of BPT: a dual inhibitor of cdk4 and tubulin polymerization. Cell Death Dis. 2015, 6, e1743.

66. Piechowska, P.; Zawirska-Wojtasiak, R.; Mildner-Szkudlarz, S. Bioactive $\beta$-carbolines in food: a review. Nutrients 2019, 11, 814.

67. Helbecque, N.; Moquin, C.; BernieR, J.L.; Morel, E., Guyot, M.; Henichart, J.P. Grossularine-1 and grossularine-2, $\alpha$-carbolines from Dendrodoa grossularia, as possible intercalative agents. Cancer Biochem. Biophys. 1987, 9, 271-279.

68. Lin, Y.-C.; Chen, Y.-F.; Tseng, L.-S.; Lee, Y.-H.; Morris-Natschke, S.L.; Kuo, S.-C.; Yang, N.-S.; Lee, K.-H.; Huang, L.-J. Synthesis and structure-activity relationship studies of novel 3,9-substituted alpha-carboline derivatives with high cytotoxic activity against colorectal cancer cells. Eur. J. Med. Chem. 2016, 110, 98-114.

69. Huang, H.-C.; Liu, W.-T.; Hua, K.-S.; Hung, H.-C.; Tsai, J.-Y.; Kuo, S.-C.; Huang, L.-J.; Gean, P.-W. $\alpha$-Carboline derivative TJY16 inhibits tumor growth by inducing G2/M cell cycle arrest in glioma cells. J. Biomed. Sci. 2016, 23, 10.

70. Valoti, G.; Nicoletti, M.I.; Pellegrino, A.; Jimeno, J.; Hendriks, H.; D’Incalci, M.; Faircloth, G.; Giavazz, R. Ecteinascidin-743, a new marine natural product with potent anti-tumor activity on human ovarian carcinoma xenografts. Clin. Cancer Res. 1998, 4, 1977-1983.

71. D'Incalci, M.; Galmarini, C.M. A review of trabectin (ET-743): A unique mechanism of action. Mol. Cancer Ther. 2010, 9, 21572163.

72. Tabunoki, H.; Saito, N.; Suwanborirux, K.; Charupant, K.; Satoh, J.-i. Molecular network profiling of U373MG human glioblastoma cells following induction of apoptosis by novel marine-derived anti-cancer 1,2,3,4-tetrahydroisoquinoline alkaloids. Cancer Cell Int. 2012, 12, 14.

73. Mure, H.; Matsuzaki, K.; Kitazato, K.T.; Mizobuchi, Y.; Kuwayama, K.; Kageji, T.; Nagahiro, S. Akt2 and Akt3 play a pivotal role in malignant gliomas. Neurooncology 2010, 12, 221-232.

74. Korur, S.; Huber, R.M.; Sivasankaran, B.; Petrich, M.; Morin, P., Jr.; Hemmings, B.A.; Merlo, A.; Lino, M.M. GSK3 regulates differentiation and growth arrest in glioblastoma. PLoS One, 2009, 4, e7443.

75. Swiss, V.A.; Casaccia, P. Cell-context specific role of the E2F/Rb pathway in development and disease. Glia 2010, 58, 377-390. 
76. Damia, G.; Silvestri, S.; Carrassa, L.; Filiberti, L.; Faircloth, G.T.; Liberi, G.; Foiani, M.; D'Incalci, M. Unique pattern of ET-743 activity in different cellular systems with defined deficiencies in DNA-repair pathways. Int. J. Cancer, 2001, 92, 583-588.

77. Kobayashi, J.; Cheng, J.F.; Kikuchi, Y.; Ishibashi, M.; Yamamura, S.; Ohizumi, Y.; Ohta, T.; Nozoe, S. Rigidin, a novel alkaloid with calmodulin antagonistic activity from the Okinawan marine tunicate Eudistoma CF rigida. Tetrahedron Lett. 1990, 31, 46174620 .

78. Davis, R.A.; Christensen, L.V.; Richardson, A.D.; da Rocha, R.M.; Ireland, C.M. Rigidin E, a new pyrrolopyrimidine alkaloid from a Papua New Guinea tunicate Eudistoma species. Mar. Drugs 2003, 1, 27-33.

79. Scott, R.; Karki, M.; Reisenauer, M.R.; Rodrigues, R.; Dasari, R.; Smith, W.R.; Pelly, S.C.; van Otterlo, W.A.L.; Shuster, C.B.; Rogelj, S. et al. Synthetic and biological studies of tubulin targeting C2-substituted 7-deazahypoxanthines derived from marine alkaloid rigidins. ChemMedChem. 2014, 9, 1428-1435.

80. Song, T.; Chen, M.; Chai, W.; Zhang, Z.; Lian, X.-Y. New bioactive pyrrospirones C-I from a marine-derived fungus Penicillium sp ZZ380. Tetrahedron 2018, 74, 884-891.

81. Song, T.; Tang, M.; Ge, H.; Chen, M.; Lian, X.; Zhang, Z. Novel bioactive penicipyrroether A and pyrrospirone J from the marine-derived Penicillium sp. ZZ380. Mar. Drugs 2019, 17, 292.

82. Chen, S.; Shen, H.; Zhang, P.; Cheng, H.; Dai, X.; Liu, L. Anti-glioma trichobamide A with an unprecedented tetrahydro-5Hfuro[2,3-b]pyrrol-5-one functionality from ascidian-derived fungus Trichobotrys effuse 4729. Chem. Commun. 2019, 55, 1438.

83. Anjum, K.; Bi, H.; Chai, W.; Lian, X.Y.; Zhang, Z. Antiglioma pseurotin A from marine Bacillus sp. FS8D regulating tumour metabolic enzymes. Nat. Prod. Res. 2018, 32, 1353-1356.

84. Baker, B.J.; Scheuer, P.J.; Shoolery, J.N. Papuamine, an antifungal pentacyclic alkaloid from a marine sponge, Haliclona sp. J. Am. Chem. Soc. 1988, 110, 965-966.

85. Fahy, E.; Molinski, T.F.; Harper, M.K.; Sullivan, B.W.; Faulkner, D.J.; Parkanyi, L.; Clardy, J. Haliclonadiamine, an antimicrobial alkaloid from the sponge Haliclona sp. Tetrahedron Lett. 1988, 29, 3427-3428.

86. Liang, Z.; Sulzmaier, F.J.; Yoshida, W.Y.; Kelly, M.; Ramos, J.W.; Williams, P.G. Neopetrocyclamines A and B, polycyclic diamine alkaloids from the sponge Neopetrosia cf exigua. J. Nat. Prod. 2015, 78, 543-547.

87. Kanno, S.-I.; Yomogida, S.; Tomizawa, A.; Yamazaki, H.; Ukai, K.; Mangindaan, R.E.P.; Namikoshi, M.; Ishikawa, M. Papuamine causes autophagy following the reduction of cell survival through mitochondrial damage and JNK activation in MCF-7 human breast cancer cells. Int. J. Oncol. 2013, 43, 1413-1419.

88. Camargo, A.J.; Oliveira, J.H.H.L.; Trsic, M.; Berlinck, R.G.S. Molecular orbital calculations, experimental and theoretical UV spectra of granulatimides and didemnimides, biologically active polycyclic heteroaromatic alkaloids from the ascidian Didemnum granulatum. J. Mol. Struct. 2001, 559, 67-77.

89. Lavrard, H.; Salvetti, B.; Mathieu, V.; Rodriguez, F.; Kiss, R.; Delfourne, E. Synthesis and in vitro antiproliferative activity of amido and amino analogues of the marine alkaloid Isogranulatimide. ChemMedChem. 2015, 10, 607-609.

90. Nweze, J.A.; Mbaoji, F.N.; Huang, G.; Li, Y.; Yang, L.; Zhang, Y.; Huang, S.; Pan, L.; Yang, D. Antibiotics development and the potentials of marine-derived compounds to stem the tide of multidrug-resistant pathogenic bacteria, fungi, and protozoa. Mar. Drugs 2020, 18, 145.

91. Tevyashova, A.N. Olivomycin A - an antitumor antibiotic of the aureolic acid group (review). Pharm. Chem. J. 2016, 50, 425430 .

92. Liu, M.; Jia, Y.; Xie, Y.; Zhang, C.; Ma, J.; Sun, C.; Ju, J. Identification of the actinomycin D biosynthetic pathway from marinederived Streptomyces costaricanus SCSIO ZS0073. Mar. Drugs 2019, 17, 240.

93. Lo, Y.S.; Tseng, W.H.; Chuang, C.Y.; Hou, M.H. The structural basis of actinomycin D-binding induces nucleotide flipping out, a sharp bend and a left-handed twist in CGG triplet repeats. Nucleic Acids Res. 2013, 41, 4284-4294.

94. Zhang, X.; Ye, X.; Chai, W.; Lian, X.-Y.; Zhang, Z. New metabolites and bioactive actinomycins from marine-derived Streptomyces sp. ZZ338. Mar. Drugs 2016, 14, 181. 
95. Tan, H.S.; Che, Q.; Li, D.H.; Gu, Q.Q.; Zhu, T.J. Progress in the research of antimycin-type compounds. Chin. J. Antibiot. 2015, $40,892-900$.

96. Hu, C.; Zhou, D.-W.; Chen, F.; Zheng, X.-H.; Shen, H.-F.; Lin, B.-R.; Zhou, G.-X. Neoantimycins A and B, two unusual benzamido nine-membered dilactones from marine-derived Streptomyces antibioticus H12-15. Molecules 2017, $22,557$.

97. Xie, G.; Zhu, X.; Li, Q.; Gu, M.; He, Z.; Wu, J.; Li, J.; Lin, Y.; Li, M.; She, Z.; Yuan, J. SZ-685C, a marine anthraquinone, is a potent inducer of apoptosis with anticancer activity by suppression of the Akt/FOXO pathway. Br. J. Pharmacol. 2010, 159, 689-697.

98. Chen, L.; Chai, W.; Wang, W.; Song, T.; Lian, X.-Y.; Zhang, Z. Cytotoxic bagremycins from mangrove-derived Streptomyces sp. Q22. J. Nat. Prod. 2017, 80, 1450-1456.

99. Hayakawa, Y.; Adachi, K.; Iwakiri, T.; Imamura, K.; Furihata, K.; Seto, H.; Otake, N. Capoamycin, a new isotetracenone antibiotic. Agric. Biol. Chem. 1987, 51, 2237-2243.

100. Xin, W.; Ye, X.; Yu, S.; Lian, X.Y.; Zhang, Z. New capoamycin-type antibiotics and polyene acids from marine Streptomyces fradiae PTZ0025. Mar Drugs 2012, 10, 2388-2402.

101. Kim, D.-G.; Moon, K.; Kim, S.-H.; Park, S.-H.; Park, S.; Lee, S.K.; Oh, K.-B.; Shin, J.; Oh, D.-C. Bahamaolides A and B, antifungal polyene polyol macrolides from the marine actinomycete Streptomyces sp. J. Nat. Prod. 2012, 75, $959-967$.

102. Wang, W.; Song, T.; Chai, W.; Chen, L.; Chen, L.; Lian, X.-Y.; Zhang, Z. Rare polyene-polyol macrolides from mangrovederived Streptomyces sp. ZQ4BG. Sci. Rep. 2017, 7, 1703.

103. Tian, X.; Tang, H.; Lin, H.; Cheng, G.; Wang, S.; Zhang, X. Saponins: the potential chemotherapeutic agents in pursuing new anti-glioblastoma drugs. Mini Rev. Med. Chem. 2013, 13, 1709-1724.

104. Cheng, G.; Zhang, X.; Tang, H-F.; Zhang, Y.; Zhang, X.-H.; Cao, W.-D.; Gao, D.-K.; Wang, X.-L.; Jin, B.-Q. Asterosaponin 1, a cytostatic compound from the starfish Culcita novaeguineae, functions by inducing apoptosis in human glioblastoma U87MG cells. J. Neurooncol. 2006, 79, 235-241.

105. Lu, Y.; Li, H.; Wang, M.; Liu, Y.; Feng, Y.; Liu. K.; Tang, H. Cytotoxic polyhydroxysteroidal glycosides from starfish Culcita novaeguineae. Mar. Drugs 2018, 16, 92.

106. Ma, N.; Tang, H.F.; Qiu, F.; Lin, H.W.; Tian, X.R.; Zhang, W. A new polyhydroxysteroidal glycoside from the starfish Anthenea chinensis. Chin. Chem. Lett. 2009, 20, 1231-1234.

107. Ma, N.; Tang, H.F.; Qiu, F.; Lin, H.W.; Tian, X.R.; Yao, M.N. Polyhydroxysteroidal glycosides from the starfish Anthenea chinensis. J. Nat. Prod., 2010, 73, 590-597.

108. Wang, M.X.; Wu, J.H.; Yi, Y.H.; Li, L.; Sun, P.; Liu, B.S.; Wang, X.D. Studies on saponins constituents of Pentaceraster semper. Chin. J. Nat. Med. 2006, 4, 344-346.

109. Han, H.; Xu, Q.Z.; Tang, H.F.; Yi, Y.H.; Gong, W. Cytotoxic holostane-type triterpene glycosides from the sea cucumber Pentacta quadrangularis. Planta Med. 2010, 76, 1900-1904.

110. Yin, Y.H.; Zhang, X.; Cheng, G.; Tang, H.F.; Lin, H. Research on suppression of fuscocineroside A on proliferation of human glioblastoma U251MG cells. Chin. J. Neurosurg. Dis. Res. 2009, 8, 431-435.

111. Yuan, W.H.; Yi, Y.H.; Tan, R.X.; Wang, Z.L.; Sun, G.Q.; Xue, M.; Zhang, H.W.; Tang, H.F. Antifungal triterpene glycosides from the sea cucumber Holothuria (Microthele) axiloga. Planta Med. 2009, 75, 647-653.

112. Sangpairoj, K.; Chaithirayanon, R.; Vivithanaporn, P.; Siangcham, T.; Jattujan, P.; Poomtong, T.; Nobsathian, S.; Sobhon, P. Extract of the sea cucumber, Holothuria scabra, induces apoptosis in human glioblastoma cell lines. Func. Foods Health Dis. 2016, $6,452-468$.

113. Yu, S.; Ye, X.; Huang, H.; Peng, R.; Su, Z.; Lian, X.-Y.; Zhang, Z. Bioactive sulfated saponins from sea cucumber Holothuria moebii. Planta Med. 2015, 81, 152-159.

114. Adao, C.R.; Pereira da Silva, B.; Tinoco, L.W.; Parente, J.P. Haemolytic activity and immunological adjuvant effect of a new steroidal saponin from Allium ampeloprasum var. porrum. Chem. Biodiv. 2012, 9, 58-67. 
115. Jiang, M.; Wu, Z.; Guo, H.; Liu, L.; Chen, S. A Review of terpenes from marine-derived fungi: 2015-2019. Mar. Drugs 2020, 18, 321.

116. Hegazy, M.-E.F.; Elshamy, A.I.; Mohamed, T.A.; Hussien, T.A.; Helaly, S.E.; Abdel-Azim, N.S.; Shams, K.A.; Shahat, A.A.; Tawfik, W.A.; Shahen, A.M. et al. Terpenoid bio-transformations and applications via cell/organ cultures: a systematic review. Crit. Rev. Biotechnol. 2020, 40, 64-82.

117. Liu Y.; Liang H.; Su A. Effect of aplysin on the proliferation and apoptosis in human gastric cancer cell SGC-7901. Chin. Pharm. Bull. 2010, 26, 333-337.

118. Zhang, X.; Zhuang, T.; Liang, Z.; Li, L.; Xue, M.; Liu, J.; Liang, H. Breast cancer suppression by aplysin is associated with inhibition of PI3K/AKT/FOXO3a pathway. Oncotarget 2017, 8, 63923-63934.

119. Gong, A.; Ge, N.; Yao, W.; Lu, L.; Liang, H. Aplysin enhances temozolomide sensitivity in glioma cells by increasing miR-181 level. Cancer Chemother. Pharmacol. 2014, 74, 531-538.

120. Gong, A.J.; Gong, L.L.; Yao, W.C.; Ge, N.; Lu, L.X.; Liang, H. Aplysin induces apoptosis in glioma cells through HSP90/AKT pathway. Exp. Biol. Med. (Maywood). 2015, 240, 639-44.

121. Chen, G.; Zhu, W.; Shi, D.; Lv, L.; Zhang, C.; Liu, P.; Hu, W. MicroRNA-181a sensitizes human malignant glioma U87MG cells to radiation by targeting Bcl-2. Oncol. Rep. 2010, 23, 997-1003.

122. Wang, J.; Sai, K.; Chen, F.R.; Chen, Z.P. miR-181b modulates glioma cell sensitivity to temozolomide by targeting MEK1. Cancer Chemother. Pharmacol. 2013, 72, 147-158.

123. Iwamaru, A.; Iwado, E.; Kondo, S.; Newman, R.A.; Vera, B.; Rodríguez, A.D.; Kondo, Y. Eupalmerin acetate, a novel anticancer agent from Caribbean gorgonian octocorals, induces apoptosis in malignant glioma cells via the c-Jun NH2terminal kinase pathway. Mol. Cancer Ther. 2007, 6, 184-192.

124. Tang, S.-A.; Zhou, Q.; Guo, W.-Z.; Qiu, Y.; Wang, R.; Jin, M.; Zhang, W.; Li, K.; Yamori, T.; Dan, S.; Kong, D. In vitro antitumor activity of stellettin B, a triterpene from marine sponge Jaspis stellifera, on human glioblastoma cancer SF295 cells. Mar. Drugs 2014, 12, 4200-4213.

125. Zhang, B.-z.; Wang, K.-r.; Yan, J.-x.; Zhang, W.; Song, J.-j.; Ni, J.-m.; Wang, R. In vitro and in vivo antitumor effects of novel actinomycin D analogs with amino acid substituted in the cyclic depsipeptides. Peptides 2010, 31, 568-573.

126. Nakamura, T.; Furunaka, H.; Miyata, T.; Tokunaga, F.; Muta, T.; Iwanaga, S.; Niwa, M.; Takao, T.; Shimonishi, Y. Tachyplesin, a class of antimicrobial peptide from the hemocytes of the horseshoe crab (Tachypleus tridentatus). Isolation and chemical structure. J. Biol. Chem. 1988, 263, 16709-16713.

127. Li, Q.F.; Ou-Yang, G.L.; Peng, X.X.; Hong, S.G. Effects of tachyplesin on the regulation of cell cycle in human hepatocarcinoma SMMC-7721 cells. World J. Gastroenterol. 2003, 9, 454-458.

128. Chen, J.; Xu, X.M.; Underhill, C.B.; Yang, S.; Wang, L.; Chen, Y.; Hong, S.; Creswell, K.; Zhang, L. Tachyplesin activates the classic complement pathway to kill tumor cells. Cancer Res. 2005, 65, 4614-4622.

129. Hoskin, D.W.; Ramamoorthy, A. Studies on anticancer activities of antimicrobial peptides. Biochim. Biophys. Acta 2008, 1778, 357-375.

130. Ding, H.; Jin, G.; Zhang, L.; Dai, J.; Dang, J.; Han, Y. Effects of tachyplesin I on human U251 glioma stem cells. Mol. Med. Rep. 2015, 11, 2953-2958.

131. Li, X.; Dai, J.; Tang, Y.; Li, L.; Jin, G. Quantitative proteomic profiling of tachyplesin I targets in U251 gliomaspheres. Mar. Drugs 2017, 15, 20.

132. Liu, Y.; Zhou, Y.; Zhu, K. Inhibition of glioma cell lysosome exocytosis inhibits glioma invasion. PLoS One 2012, 7, e45910.

133. Medina, R.A.; Goeger, D.E.; Hills, P.; Mooberry, S.L.; Huang, N.; Romero, L.I.; Ortega-Barrı, E.; Gerwick, W.H.; McPhail, K.L. Coibamide A, a potent antiproliferative cyclic depsipeptide from the Panamanian marine cyanobacterium Leptolyngbya sp. J. Am. Chem. Soc. 2008, 130, 6324-6325.

134. Hau, A.M.; Greenwood, J.A.; Löhr, C.V.; Serrill, J.D.; Proteau, P.J.; Ganley, I.G.; McPhail, K.L.; Ishmael, J.E. Coibamide A Induces mTOR-Independent Autophagy and Cell Death in Human Glioblastoma Cells. PLoS One 2013, 8, e65250. 
135. Serrill, J.D.; Wan, X.; Hau, A.M.; Jang, H.S.; Coleman, D.J.; Indra, A.K.; Alani, A.W.G.; McPhail, K.L.; Ishmael, J.E. Coibamide $\mathrm{A}$, a natural lariat depsipeptide, inhibits VEGFA/VEGFR2 expression and suppresses tumor growth in glioblastoma xenografts. Invest. New Drugs 2016, 34, 24-40.

136. Brennan, C. Genomic profiles of glioma. Curr. Neurol. Neurosci. Rep. 2011, 11, 291-297.

137. Ye, X.; Anjum, K.; Song, T.; Wang, W.; Liang, Y.; Chen, M.; Huang, H.; Lian, X.-Y.; Zhang, Z. Antiproliferative cyclodepsipeptides from the marine actinomycete Streptomyces sp. P11-23B downregulating the tumor metabolic enzymes of glycolysis, glutaminolysis, and lipogenesis. Phytochemistry, 2017, 135, 151-159.

138. Dembitsky, V.M. Antitumor and hepatoprotective activity of natural and synthetic neo steroids. Prog. Lipid Res. 2020, 79 , 101048.

139. Quang, T.H.; Lee, D.-S.; Han, S.J.; Kim, I.C.; Yim, J.H.; Kim, Y.-C.; Oh, H. Steroids from the cold water starfish Ctenodiscus crispatus with cytotoxic and apoptotic effects on human hepatocellular carcinoma and glioblastoma cells. Bull. Korean Chem. Soc. 2014, 35, 2335.

140. Blunt, J.W.; Copp, B.R.; Keyzers, R.A.; Munro, M.H.G.; Prinsep, M.R. Marine natural products. Nat. Prod. Rep. 2015, 32, 116211.

141. Zhang, Y.-M.; Li, H.-Y.; Hu, C.; Sheng, H.-F.; Zhang, Y.; Lin, B.-R.; Zhou, G.-X. Ergosterols from the culture broth of marine Streptomyces anandii H41-59. Mar. Drugs 2016, 14, 84.

142. Yu, S.; Ye, X.; Chen, L.; Lian, X.-Y.; Zhang, Z. Polyoxygenated 24, 28-epoxyergosterols inhibiting the proliferation of glioma cells from sea anemone Anthopleura midori. Steroids 2014, 88, 19-25.

143. Chen, M.; Chai, W.; Song, T.; Ma, M.; Lian, X.-Y.; Zhang, Z. Anti-glioma natural products downregulating tumor glycolytic enzymes from marine actinomycete Streptomyces sp. ZZ406. Sci. Rep. 2018, 8, 72.

144. Schneemann, I.; Kajahn, I.; Ohlendorf, B.; Zinecker, H.; Erhard, A.; Nagel, K.; Wiese, J.; Imhoff, J.F. Mayamycin, a cytotoxic polyketide from a Streptomyces strain isolated from the marine sponge Halichondria panicea. J. Nat. Prod. 2010, 73, 1309-1312.

145. Liang, Y.; Xie, X. Chen, L.; Yan, S.; Ye, X.; Anjum, K.; Huang, H.; Lian, X.; Zhang, Z. Bioactive polycyclic quinones from marine Streptomyces sp. 182SMLY. Mar. Drugs 2016, 14, 10.

146. Wright, A.D.; Nielson, J.L.; Tapiolas, D.M.; Motti, C.A.; Ovenden, S.P.B.; Kearns, P.S.; Liptrot, C.H. Detailed NMR, including 1,1-ADEQUATE, and anticancer studies of compounds from the echinoderm Colobometra perspinosa. Mar. Drugs 2009, 7, 565575.

147. Wätjen, W.; Ebada, S.S.; Bergermann, A.; Chovolou, Y.; Totzke, F.; Kubbutat, M.H.G.; Li, W.; Proksch, P. Cytotoxic effects of the anthraquinone derivatives 1'-deoxyrhodoptilometrin and (S)-(-)-rhodoptilometrin isolated from the marine echinoderm Comanthus sp. Arch. Toxicol. 2017, 91, 1485-1495.

148. Guinan, M.; Benckendorff, C.; Smith, M.; Miller, G.F. Recent advances in the chemical synthesis and evaluation of anticancer nucleoside analogues. Molecules 2020, 25, 2050.

149. Searle P.A.; Molinski, T.F. Trachycladine-A and trachycladine-B-2'-C-methyl-5'-deoxyribofuranosyl nucleosides from the marine sponge Trachycladus laevispirulifer. J. Org. Chem. 1995, 60, 4296-4298.

150. Peitsinis, Z.V.; Mitrakas, A.G.; Nakiou, E.A.; Melidou, D.A.; Kalamida, D.; Kakouratos, C.; Koukourakis, M.I.; Koumbis, A.E. Trachycladines and analogues: synthesis and evaluation of anticancer activity. ChemMedChem. 2017, 12, 448-455.

151. Cheng-Sánchez, I.; Sarabia, F. Chemistry and biology of bioactive glycolipids of marine origin. Mar. Drugs 2018, 16, 294.

152. Jacob, F.; Alam, S.; Konantz, M.; Liang, C.-Y.; Kohler, R. S.; Everest-Dass, A. V.; Huang, Y.-L.; Rimmer, N.; Fedier, A.; Schotzau, A. et al. Transition of mesenchymal and epithelial cancer cells depends on alpha 1-4 galactosyltransferase-mediated glycosphingolipids. Cancer Res. 2018, 78, 2952-2965.

153. Furukawa, K.; Ohmi, Y.; Ohkawa, Y.; Bhuiyan, R.H.; Zhang, P.; Tajima, O.; Hashimoto, N.; Hamamura, K.; Furukawa, K. New era of research on cancer- associated glycosphingolipids. Cancer Sci. 2019, 110, 1544-1551.

154. Farokhi, F.; Wielgosz-Collin, G.; Clément, M.; Kornprobst, J.M.; Barnathan, G. Cytotoxicity on human cancer cells of ophidiacerebrosides isolated from the African starfish Narcissia canariensis. Mar. Drugs 2010, 8, 2988-2998. 
155. Farokhi, F.; Grellier, P.; Clément, M.; Roussakis, C.; Loiseau, P.M.; Genin-Seward, E.; Kornprobst, J.-M.; Barnathan, G.; Wielgosz-Collin, G. Antimalarial activity of axidjiferosides, new $\beta$-galactosylceramides from the African sponge Axinyssa djiferi. Mar. Drugs 2013, 11, 1304-1315.

156. Biegelmeyer, R.; Schröder, R.; Rambo, D.F.; Dresch, R.R.; Carraro, J.L.F.; Mothes, B.; Moreira, J.C.F.; Frota, M.L.C.; Henriques, A.T. Sphingosines derived from marine sponge as potential multi-target drug related to disorders in cancer development. Mar. Drugs 2015, 13, 5552-5563.

157. Woodcock, J. Sphingosine and ceramide signaling in apoptosis. Life 2006, 58, 462-466.

158. Huang, C.; Freter, C. Lipid metabolism, apoptosis and cancer therapy. Int. J. Mol. Sci. 2015, 16, $924-949$.

159. Bernhart, E.; Damm, S.; Wintersperger, A.; Nusshold, C.; Brunner, A.M.; Plastira, I.; Rechberger, G.; Reicher, H.; Wadsack, C.; Zimmer, A. et al. Interference with distinct steps of sphingolipid synthesis and signaling attenuates proliferation of U87MG glioma cells. Biochem. Pharmacol. 2015, 96, 119-130.

160. Quiňoà, E.; Crew, C.P. Phenolic constituents of psammaplysilla. Tetrahedron Lett. 1987, 28, 3229-3233.

161. Hoshino, O.; Murakata, M.; Yamada, K. A convenient synthesis of a bromotyrosine derived metabolite, psammaplin A, from psammaplysilla sp. Bioorg. Med. Chem. Lett. 1992, 2, 1561-1562.

162. Zhou, Y.-D.; Li, J.; Du, L.; Mahdi, F.; Le, T.P.; Chen, W.-L.; Swanson, S.M.; Watabe, K.; Nagle, D.G. Biochemical and anti-triple negative metastatic breast tumor cell properties of psammaplins. Mar. Drugs 2018, 16, 442.

163. Jing, Q.; Hu, X.; Ma, Y.; Mu, J.; Liu, W.; Xu, F.; Li, Z.; Bai, J.; Hua, H.; Dahong L. Marine-derived natural lead compound disulfide-linked dimer psammaplin A: biological activity and structural modification. Mar. Drugs 2019, $17,384$.

164. Ratovitski, E.A. Tumor Protein (TP)-p53 members as regulators of autophagy in tumor cells upon marine drug exposure. Mar. Drugs 2016, 14, 154.

165. Mora, F.D.; Jones, D.K.; Desai, P.V.; Patny, A.; Avery, M.A.; Feller, D.R.; Smillie, T.; Zhou, Y.D.; Nagle, D.G. Bioassay for the identification of natural product-basedactivators of peroxisome proliferator-activated receptor- $\gamma$ (PPAR $\gamma$ ): The marine sponge metabolite psammaplin A activates PPAR $\gamma$ and induces apoptosis in human breast tumor cells. J. Nat. Prod. 2006, 69, 547-552.

166. Kim, H.J.; Kim, J.H.; Chie, E.K.; Young, P.D.; Kim, I.A.; Kim, I.H. DNMT (DNA methyltransferase) inhibitors radiosensitize human cancer cells by suppressing DNA repair activity. Radiat. Oncol. 2012, 7, 39.

167. Dote, H.; Cerna, D.; Burgan, W.E.; Carter, D.J.; Cerra, M.A.; Hollingshead, M.G.; Camphausen, K.; Tolifon, P.J. Enhancement of in vitro and in vivo tumor cell radiosensitivity by the DNA methylation inhibitor zebularine. Clin. Cancer Res. 2005, 11, 4571-4579.

168. Qiu, H.; Yashiro, M.; Shinto, O.; Matsuzaki, T.; Hirakawa, K. DNA methyltransferase inhibitor 5-aza-CdR enhances the radiosensitivity of gastric cancer cells. Cancer Sci. 2009, 100, 181-188.

169. Kim, T.H.; Kim, H.S.; Kang, Y.J.; Yoon, S.; Lee, J.; Choi, W.S.; Jung, J.H.; Kim, H.S. Psammaplin A induces sirtuin 1-dependent autophagic cell death in doxorubicin-resistant MCF-7/adr human breast cancer cells and xenografts. Biochim. Biophys. Acta. 2015, 1850, 401-410.

170. Liu, J.; Debnath, J. The evolving, multifaceted roles of autophagy in cancer. Adv. Cancer Res. 2016, 130, 1-53.

171. Sui, X.; Jin, L.; Huang, X.; Geng, S.; He, C.; Hu, X. p53 signaling and autophagy in cancer: A revolutionary strategy could be developed for cancer treatment. Autophagy 2011, 7, 565-571.

172. Huang, Y.; Ratovitski, E.A. Phospho-DNp63/Rpn13-dependent regulation of LKB1 degradation modulates autophagy in cancer cells. Aging 2010, 2, 959-968.

173. Riganti, C.; Salaroglio, I.C.; Caldera, V.; Campia, I.; Kopecka, J.; Mellai, M.; Annovazzi, L.; Bosia, A.; Ghigo, D.; Schiffer, D. Temozolomide downregulates P-glycoprotein expression in glioblastoma stem cells by interfering with the Wnt3a/glycogen synthase-3 kinase/ b-catenin pathway. Neurooncol. 2013, 15, 1502-1517.

174. Munoz, J.L.; Wlaker, N.D.; Scotto, K.W.; Rameshwar, P. Temozolomide competes for P-glycoprotein and contributes to chemoresistance in glioblastoma cells. Cancer Lett. 2015, 367, 69-75. 
175. Salaroglio, I.C.; Mujumdar, P.; Annovazzi, L.; Kopecka, J.; Mellai, M.; Schiffer, D.; Poulsen, S.-A.; Riganti, C. Carbonic Anhydrase XII Inhibitors Overcome P-Glycoprotein-Mediated Resistance to Temozolomide in Glioblastoma. Mol. Cancer Ther. 2018, 17, 2598-2609.

176. Kopecka, J.; Rankin, G.M.; Salaroglio, I.C.; Poulsen, S.A.; Riganti, C. P-glycoprotein-mediated chemoresistance is reversed by carbonic anhydrase XII inhibitors. Oncotarget 2016, 7, 85861-85875.

177. Jiménez, C.; Crews, P. Novel marine sponge derived amino acids. 13. Additional psammaplin derivatives from Psammaplysilla purpurea. Tetrahedron, 1991, 47, 2097-2102.

178. Mujumdar, P.; Teruya, K.; Tonissen, K.F.; Vullo, D.; Supuran, C.T.; Peat, T.S.; Poulsen, S.-A. An unusual natural product primary sulfonamide: synthesis, carbonic anhydrase inhibition, and protein Xray structures of psammaplin C. J. Med. Chem. 2016, 59, 5462-5470.

179. Mujumdar, P.; Kopecka, J.; Bua, S.; Supuran, C.T.; Riganti, C.; Poulsen, S.A. Carbonic anhydrase XII inhibitors overcome temozolomide resistance in glioblastoma. J. Med. Chem. 2019, 62, 4174-4192.

180. Krohn, K.; Riaz, M.; Flörke, U. Synthesis of xyloketals, natural products from the mangrove fungus Xylaria sp. Eur. J. Org. Chem. 2004, 2004, 1261-1270.

181. Gong, H.; Luo, Z.; Chen, W.; Feng, Z.-P.; Wang, G.-L.; Wang, G.-L.; Sun, H.-S. Marine compound xyloketal B as a potential drug development target for neuroprotection. Mar. Drugs 2018, 16, 516.

182. Chen, W.-L.; Turlova, E.; Sun, C.L.F.; Kim, J.-S.; Huang, S.; Zhong, X.; Guan, Y.-Y.; Wang, G.-L.; Rutka, J.T.; Feng, Z.-P.; Sun, H.-S. Xyloketal B suppresses glioblastoma cell proliferation and migration in vitro through inhibiting TRPM7-regulated PI3K/Akt and MEK/ERK signaling pathways. Mar. Drugs 2015, 13, 2505-2525.

183. Mandal, A.K.; Taniguchi, M.; Diers, J.R.; Niedzwiedzki, D.M.; Kirmaier, C.; Lindsey, J.S.; Bocian, D.F.; Holten, D. Photophysical properties and electronic structure of porphyrins bearing zero to four meso-phenyl substituents: New insights into seemingly well understood tetrapyrrols. J. Phys. Chem. A, 2016, 120, 9719-9731.

184. Cho, M.; Park, G.M.; Kim, S.N.; Amna, T.; Lee, S.; Shin, W.S. Glioblastoma-specific anticancer activity of pheophorbide a from the edible red seaweed Grateloupia elliptica. J. Microbiol. Biotechnol. 2014, 24, 346-353.

185. Searle, P.A.; Molinski, T.F.; Brzezinski, L.J.; Leahy, J.W. Absolute configuration of phorboxazoles A and B from the marine sponge Phorbas sp. 1. Macrolide and Hemiketal Rings. J. Am. Chem. Soc. 1996, 118, 9422-9423.

186. Forsyth, C.J.; Ahmed, F.; Cink, R.D.; Lee, C.S. Total synthesis of phorboxazole A. J. Am. Chem. Soc. 1998, 120, $5597-5598$.

187. Uckun, F.M.; Forsyth, C.J. Anticancer activity of synthetic analogues of the phorboxazoles. Bioorg. Med. Chem. Lett. 2001, 11, 1181-1183.

188. Thomas, N.V.; Kim, S.-K. Potential pharmacological applications of polyphenolic derivatives from marine brown algae. Environ. Toxicol. Pharmacol. 2011, 32, 325-335.

189. Manandhar, B.; Paudel, P.; Seong, S.H.; Jung, H.A.; Choi, J.S. Characterizing eckol as a therapeutic aid: A systematic review. Mar. Drugs 2019, 17, 361.

190. Hyun, K.H.; Yoon, C.H.; Kim, R.K.; Lim, E.J.; An, S.; Park, M.J.; Hyun, J.W.; Suh, Y.; Kim, M.J.; Lee, S.J. Eckol suppresses maintenance of stemness and malignancies in glioma stem-like cells. Toxicol. Appl. Pharmacol. 2011, 254, 32-40.

191. Stiles, C.D.; Rowitch, D.H. Glioma stem cells: a midterm exam. Neuron, 2008, 58, 832-846.

192. Pevny, L.H.; Nicolis, S.K. Sox2 roles in neural stem cells. Int. J. Biochem. Cell Biol. 2010, 42, 421-424.

193. Hjelmeland, A.B.; Wu, Q.; Wickman, S.; Eyler, C.; Heddleston, J.; Shi, Q.; Lathia, J.D.; Macswords, J.; Lee, J.; McLendon, R.E.; Rich, J.N. Targeting A20 decreases glioma stem cell survival and tumor growth. PLoS Biol. 2010. 8, e1000319.

194. Lu, D.Y.; Chang, C.S.; Yeh, W.L.; Tang, C.H.; Cheung, C.W.; Leung, Y.M.; Liu, J.F.; Wong, K.L. The novel phloroglucinol derivative BFP induces apoptosis of glioma cancer through reactive oxygen species and endoplasmic reticulum stress pathways. Phytomedicine 2012, 19, 1093-1100.

195. Almeida, T.; Ramos, A.A.; Ferreira, J.; Azqueta, A.; Rocha, E. Bioactive compounds from seaweed with anti-leukemic activity: a mini-review on carotenoids and phlorotannins. Mini Rev. Med. Chem. 2020, 20, 39-53. 
196. Kim, S.M.; Kang, S.-W.; Kwon, O.-N.; Chung, D.; Pan, C.-H. Fucoxanthin as a major carotenoid in Isochrysis aff. Galbana: characterization of extraction for commercial application. J. Korean Soc. Appl. Biol. Chem. 2012, 55, 477-483.

197. Mikami, K.; Hosokawa, M. Biosynthetic pathway and health benefits of fucoxanthin, an algae-specific xanthophyll in brown seaweeds. Int. J. Mol. Sci. 2013, 14, 13763-13781.

198. Mohamadnia, S.; Tavakoli, O.; Faramarzi, M.A.; Shamsollahi, Z. Production of fucoxanthin by the microalga Tisochrysis lutea: A review of recent developments. Aquaculture, 2020, 516, 734637.

199. Yousefi, M.K.; Hashtroudi, M.S.; Moradi, A.M.; Ghasempour, A.R. In vitro investigating of anticancer activity of focuxanthin from marine brown seaweed species. Glob. J. Environ. Sci. Manag. 2018, 4, 81-90.

200. Liu, Y.; Zheng, J.; Zhang, Y.; Wang, Z.; Yang. Y.; Bai, M.; Dai, Y. Fucoxanthin activates apoptosis via inhibition of $\mathrm{PI} 3 \mathrm{~K} / \mathrm{Akt} / \mathrm{mTOR}$ pathway and suppresses invasion and migration by restriction of $\mathrm{p} 38-\mathrm{MMP}-2 / 9$ pathway in human glioblastoma cells. Neurochem Res. 2016, 41, 2728-2751.

201. Author 1, A.B.; Author 2, C.D. Title of the article. Abbreviated Journal Name Year, Volume, page range.

202. Author 1, A.; Author 2, B. Title of the chapter. In Book Title, 2nd ed.; Editor 1, A., Editor 2, B., Eds.; Publisher: Publisher Location, Country, 2007; Volume 3, pp. 154-196.

203. Author 1, A.; Author 2, B. Book Title, 3rd ed.; Publisher: Publisher Location, Country, 2008; pp. $154-196$.

204. Author 1, A.B.; Author 2, C. Title of Unpublished Work. Abbreviated Journal Name stage of publication (under review; accepted; in press).

205. Author 1, A.B. (University, City, State, Country); Author 2, C. (Institute, City, State, Country). Personal communication, 2012.

206. Author 1, A.B.; Author 2, C.D.; Author 3, E.F. Title of Presentation. In Title of the Collected Work (if available), Proceedings of the Name of the Conference, Location of Conference, Country, Date of Conference; Editor 1, Editor 2, Eds. (if available); Publisher: City, Country, Year (if available); Abstract Number (optional), Pagination (optional).

207. Author 1, A.B. Title of Thesis. Level of Thesis, Degree-Granting University, Location of University, Date of Completion.

208. Title of Site. Available online: URL (accessed on Day Month Year). 